CENTRO UNIVERSITÁRIO FEI

CAMILA RESTANI ALVES

ANÁLISE DE DESCASAMENTO NAS CARACTERÍSTICAS ELÉTRICAS DE SOI nMOSFET DE CANAL GRADUAL OPERANDO EM SATURAÇÃO

São Bernardo do Campo 


\section{ANÁLISE DE DESCASAMENTO NAS CARACTERÍSTICAS ELÉTRICAS DE SOI nMOSFET DE CANAL GRADUAL OPERANDO EM SATURAÇÃO}

Dissertação de Mestrado apresentada ao Centro Universitário FEI, como parte dos requisitos necessários para obtenção do título de Mestre em Engenharia Elétrica. Orientada pela Prof ${ }^{a}$. Dra. Michelly de Souza.

São Bernardo do Campo 
Restani Alves, Camila.

Análise de Descasamento nas Características Elétricas de SOI nMOSFET de Canal Gradual Operando em Saturação / Camila Restani Alves. São Bernardo do Campo, 2017.

$114 \mathrm{f}$ : : il.

Dissertação - Centro Universitário FEI.

Orientadora: Prof. ${ }^{a}$ Dra. Michelly de Souza.

1. Transistor SOI nMOSFET. 2. Canal Gradual. 3. Saturação. 4. Descasamento. I. de Souza, Michelly, orient. II. Título.

Elaborada pelo sistema de geração automática de ficha catalográfica da FEI com os dados fornecidos pelo(a) autor(a). 
Aluno: Camila Restani Alves

Matrícula: $115124-0$

Título do Trabalho: Análise de descasamento nas características elétricas de SOI nMOSFET de canal gradual operando em saturação.

Área de Concentração: Dispositivos Eletrônicos Integrados

Orientador: Prof.a Dr.a Michelly de Souza

Data da realização da defesa: $28 / 06 / 2017$

\section{ORIGINAL ASSINADA}

Avaliação da Banca Examinadora:

São Bernardo do Campo, 28 / 06 / 2017.

\section{MEMBROS DA BANCA EXAMINADORA}

Prof.a Dr.a Michelly de Souza

Prof. Dr. Genaro Mariniello da Silva

Prof. Dr. Rudolf Theoderich Bühler
Ass.:

Ass.:

Ass.:

A Banca Julgadora acima-assinada atribuiu ao aluno o seguinte resultado:

APROVADO $\bigotimes$

REPROVADO

\section{VERSÃO FINAL DA DISSERTAÇÃO}

APROVO A VERSÄO FINAL DA DISSERTAÇÄO EM QUE FORAM INCLUÍDAS AS RECOMENDAÇÕES DA BANCA EXAMINADORA
Aprovação do Coordenador do Programa de Pós-graduação

Prof. Dr. Carlos Eduardo Thomaz 
Dedico este trabalho aos meus pais, que sempre me apoiaram e estiveram lá por mim. 


\section{AGRADECIMENTOS}

Agradeço a Deus por me ajudar a superar as dificuldades encontradas ao longo do trabalho.

Aos meus pais pelo constante incentivo e apoio em minhas escolhas.

Agradeço à minha orientadora Prof ${ }^{a}$. Dra. Michelly de Souza, pela contínua atenção, paciência, apoio e dedicação, os quais me fizeram crescer e evoluir.

Aos professores, Dr. Marcelo Pavanello, Dr. Salvador Gimenez, Dr. Rodrigo Doria, Dr. Renan Doria e Dr. Rudolf Bühler, pelos ensinamentos.

Agradeço ao Centro Universitário FEI por disponibilizar toda a infraestrutura necessária à realização das atividades de pesquisa.

À CAPES, pelo suporte financeiro, permitindo a realização deste trabalho.

E tantas outras pessoas, que de forma involuntária, foram aqui omitidas. 


\section{RESUMO}

Os transistores da tecnologia Silício-sobre-Isolante (SOI - Silicon-On-Insulator) apresentam vantagens em relação à tecnologia CMOS (Complementary Metal-Oxide-Semiconductor) convencional (BULK), tais como diminuição do efeito de canal curto, aumento da transcondutância, aumento da mobilidade, entre outras. Entretanto, apresentam reduzida tensão de ruptura de dreno, devido à ativação do transistor bipolar parasitário inerente à estrutura SOI. Com o intuito de diminuir esses efeitos indesejados foi projetada uma estrutura MOSFET chamada de SOI de Canal Gradual (GC SOI - Graded-Channel). Resultados reportados na literatura mostram que GC SOI MOSFETs apresentam uma redução importante da condutância de saída, quando comparados aos SOI MOSFETs convencionais de mesma dimensão, além de aumento da transconduância e tensão de ruptura, qualificando estes transistores par aplicações analógicas de alto desempenho. Durante o processo de fabricação de transistores idênticos, devido às etapas do processo, pode ocorrer o descasamento nas suas características. A semelhança entre esses dispositivos é importante para o bom funcionamento de circuitos integrados elétricos que consideram dispositivos idênticos, tais como em um par diferencial e espelho de corrente. Este trabalho tem como obejtivo realizar o estudo do descasamento ("mismatching") das características elétricas de SOI MOSFETs de canal gradual, comparando-os a transistores com canal uniformemente dopado. Serão analisados parâmetros elétricos básicos e analógicos, tais como a tensão de limiar, transcondutância, condutância de saída, tensão Early e ganho de tensão.

Palavras-chave: SOI. MOSFETs. Canal Gradual. Descasamento. Características Analógicas. 


\begin{abstract}
Silicon-On-Insulator technology (SOI) show advantages when compared to conventional Complementary Metal-Oxide-Semiconductor (CMOS BULK) technology such as reduction of short channel effect, increase of transconductance and mobility, among others. However, it shows reduced drain breakdown voltage, due to the activation of the parasitic bipolar transistor inherent from the SOI structure. With the aim of decreasing these undesirable effects a new MOSFET structure called Graded-Channel SOI (GC SOI) has been proposed. Results reported in the literature show that GC SOI MOSFETs have an important decrease of the output conductance, when compared to conventional SOI MOSFETs of the same dimensions, besides increased transconductance and breakdown voltage, qualifying this transistor for high performance analog applications. During the process fabrication of identical transistors, due to process steps, there might be mismatching between them. The matching between devices is important to ensure good performance of analog integrated circuits that rely on identical devices, such as differential pairs and current mirrors. This work aims to study electrical characteristics mismatching of GC SOI MOSFET, comparing them with uniform channel doped transistors. Basic and analog electrical parameters, such as threshold voltage, transconductance, output conductance, Early voltage and intrinsic voltage gain will be analyzed.
\end{abstract}

Keywords: SOI. Graded Channel. Mismatching. Analog Characteristics. 


\section{LISTA DE ILUSTRAÇÕES}

Figura 1 - Perfil transversal de um SOI nMOSFET. .......................................................... 22

Figura 2 - Diagrama de faixas de energia do SOI MOSFET de camada espessa................... 23

Figura 3 - Diagrama de faixas de energia do SOI MOSFET de camada fina......................... 24

Figura 4 - Extração da inclinação de sublimiar da curva IDS em função de $\mathrm{V}_{\mathrm{GS}}$..................... 29

Figura 5 - Inclinação de sublimiar de um transistor SOI nMOSFET totalmente depletado

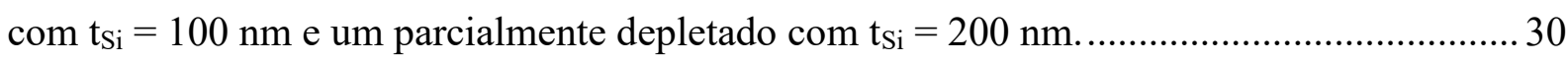

Figura 6 - Perfil transversal de MOSFETs convencionais................................................. 31

Figura 7 - Tensão de limiar em função do comprimento de canal de um dispositivo Bulk e

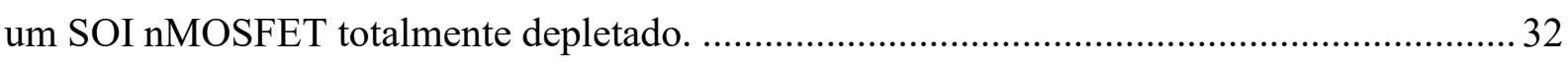

Figura 8 - Perfil transversal de SOI MOSFETs totalmente depletados................................. 32

Figura 9 - Amplificador de tensão com um único transistor MOS........................................ 34

Figura 10 - Representação esquemática da razão $g_{m} / I_{D S}$ de MOSFETs. ................................ 36

Figura 11 - Determinação da tensão Early através da extrapolação das curvas de corrente de dreno na saturação em função da tensão de dreno.............................................................. 38

Figura 12 - Perfil transversal de um SOI nMOSFET sofrendo modulação do comprimento

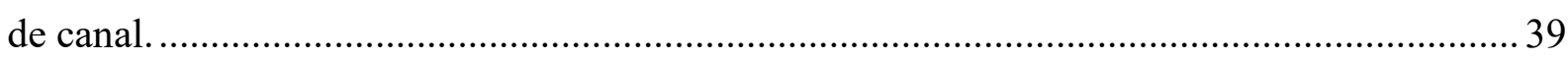

Figura 13 - Perfil de um GC SOI MOSFET totalmente depletado........................................ 43

Figura 14 - Curvas da transcondutância em função da tensão aplicada à porta. ..................... 44

Figura 15 - Curvas IDS em função de $V_{D S}$ de dispositivos nMOSFETs.................................. 45

Figura $16-\sigma_{0} \mathrm{~V}_{\text {thf }} / \mathrm{V}_{\text {thf,mean }}$ em função da razão $1 /\left(\mathrm{W} x \mathrm{~L}_{\mathrm{eff}}\right)^{1 / 2}$ para GC SOI MOSFETs

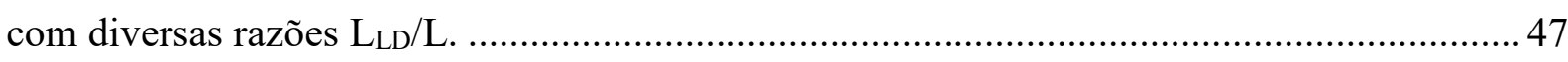

Figura 17 - Desvio relativo da corrente de dreno em função da corrente normalizada

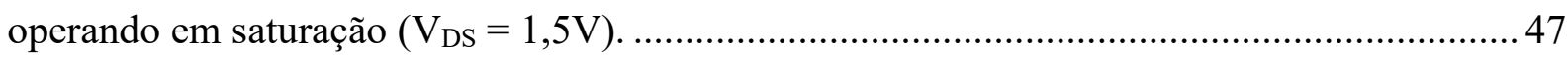

Figura 18 - Foto parcial do chip contendo os SOI MOSFETs utilizados. .............................. 48

Figura 19 - $\mathrm{I}_{\mathrm{DS} \text {,mean }}$ em função de $\mathrm{V}_{\mathrm{GF}}$ para os GC SOI MOSFETs com $\mathrm{V}_{\mathrm{DS}}=25 \mathrm{mV}$ e

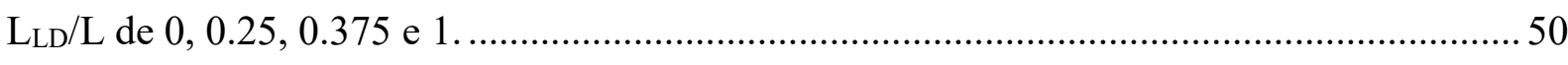

Figura 20 - IDS,mean em função de $\mathrm{V}_{\mathrm{GF}}$ em escala logarítmica para os GC SOI MOSFETs com $\mathrm{V}_{\mathrm{DS}}=25 \mathrm{mV}$ e $\mathrm{L}_{\mathrm{LD}} / \mathrm{L}$ de $0,0.25,0.375$ e 1 .

Figura $21-\sigma \mathrm{V}_{\text {thf }} / \mathrm{V}_{\text {thf,mean }}$ em função da relação $1 /\left(\mathrm{W} \text { x L } \mathrm{L}_{\text {eff }}\right)^{1 / 2}$ para transistores GC SOI e

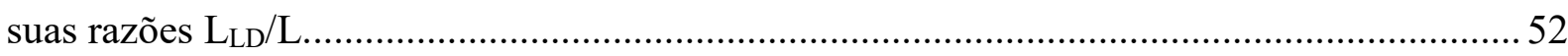

Figura 22 - Desvio padrão relativo da corrente de dreno em função da sobretensão de condução com tensão de dreno $\mathrm{V}_{\mathrm{DS}}$ de $25 \mathrm{mV}$. 
Figura 23 - Desvio padrão relativo da corrente de dreno em função da sobretensão de condução com tensão de dreno $\mathrm{V}_{\mathrm{DS}}$ de $1,5 \mathrm{~V}$.

Figura 24 - Transcondutância média em função da tensão de condução $\mathrm{V}_{\mathrm{DS}}$ de $25 \mathrm{mV}$..........55

Figura 25 - Transcondutância média em função da tensão de condução com $V_{D S}$ de 1,5 V...55

Figura 26 - Desvio padrão relativo da transcondutância em função da tensão de condução

com tensão de dreno $\mathrm{V}_{\mathrm{DS}}$ de $25 \mathrm{mV}$.

Figura 27 - Desvio padrão relativo da transcondutância em função da tensão de condução com tensão de dreno $\mathrm{V}_{\mathrm{DS}}$ de $1,5 \mathrm{~V}$.

Figura 28 - Corrente de dreno média (IDS,mean) em função de $V_{D S}$ para transistores SOI de canal uniformemente dopado e gradual polarizados com $\mathrm{V}_{\mathrm{GT}}=200 \mathrm{mV}$

Figura 29 - Corrente de dreno média (IDS,mean) em função de VDs para transistores SOI de canal uniformemente dopado e gradual polarizados com $\mathrm{V}_{\mathrm{GT}}=800 \mathrm{mV}$

Figura 30 - Desvio padrão da corrente de dreno em função da tensão de dreno com sobretensão de condução de 200 e 800 mV.

Figura 31 - Tensão Early em função da tensão de dreno com sobretensão de condução de $200 \mathrm{mV}$.

Figura 32 - Desvio da Tensão Early em função da tensão de dreno com sobretensão de condução de $200 \mathrm{mV}$.

Figura 33 - Curva $\mathrm{I}_{\mathrm{DS} \text {,mean }}$ em função de $\mathrm{V}_{\mathrm{GT}}$ para os GC SOI MOSFETs com L $\mathrm{L}_{\mathrm{HD}}$ variando $\pm 30 \mathrm{~nm}, \mathrm{~V}_{\mathrm{DS}}=50 \mathrm{mV}$ e $\mathrm{L}_{\mathrm{LD}} / \mathrm{L}$ de $0.2,0.3,0.4,0.5$.

Figura 34 - Curva IDS,mean em função de $\mathrm{V}_{\mathrm{GT}}$ para os GC SOI MOSFETs com L $\mathrm{L}_{\mathrm{HD}}$ variando $\pm 30 \mathrm{~nm}, \mathrm{~V}_{\mathrm{DS}}=1,5 \mathrm{~V}$ e $\mathrm{L}_{\mathrm{LD}} / \mathrm{L}$ de $0.2,0.3,0.4,0.5$.

Figura 35 - Curva $\mathrm{I}_{\mathrm{DS} \text {,mean }}$ em função de $\mathrm{V}_{\mathrm{GT}}$ para os GC SOI MOSFETs com $\mathrm{L}_{\mathrm{HD}}$ variando $\pm 30 \mathrm{~nm}, \mathrm{~V}_{\mathrm{DS}}=3 \mathrm{~V}$ e $\mathrm{L}_{\mathrm{LD}} / \mathrm{L}$ de $0.2,0.3,0.4,0.5$.

Figura 36 - Desvio relativo da corrente de dreno em função da sobretensão de condução de com $\mathrm{V}_{\mathrm{DS}}=50 \mathrm{mV}(\mathrm{A}), 1,5 \mathrm{~V}$ (B) e $3 \mathrm{~V}(\mathrm{C})$, com $\mathrm{L}_{\mathrm{HD}}$ variando $\pm 30 \mathrm{~nm}$

Figura 37 - Desvio relativo da transcondutância em função da sobretensão de condução de

$\mathrm{V}_{\mathrm{DS}}=50 \mathrm{mV}(\mathrm{A}), 1,5 \mathrm{~V}(\mathrm{~B})$ e $3 \mathrm{~V}(\mathrm{C})$, com $\mathrm{L}_{\mathrm{HD}}$ variando $\pm 30 \mathrm{~nm}$.

Figura 38 - Curva IDs em função de $\mathrm{V}_{\mathrm{DS}}$ com sobretensão de condução $\mathrm{V}_{\mathrm{GT}}=400 \mathrm{mV}$ com $\mathrm{L}_{\mathrm{HD}}$ variando $\pm 30 \mathrm{~nm}$

Figura 39 - Desvio relativo da corrente de dreno em função de $\mathrm{V}_{\mathrm{DS}}$ com sobretensão de condução $\mathrm{V}_{\mathrm{GT}}=0 \mathrm{mV}$ (A), $200 \mathrm{mV}$ (B), $400 \mathrm{mV}$ (C), $600 \mathrm{mV}$ (D) e $800 \mathrm{mV}$ (E), com $\mathrm{L}_{\mathrm{HD}}$ variando $\pm 30 \mathrm{~nm}$ 
Figura 40 - Tensão Early em função da tensão de dreno com sobretensão de condução $\mathrm{V}_{\mathrm{GT}}$ $=200 \mathrm{mV}$ com $\mathrm{L}_{\mathrm{HD}}$ variando $\pm 30 \mathrm{~nm}$.

Figura 41 - Curva $\mathrm{I}_{\mathrm{DS} \text {,mean }}$ em função de $\mathrm{V}_{\mathrm{GT}}$ para os GC SOI MOSFETs com concentração de $5.10^{16} \mathrm{~cm}^{-3}$ variando $\pm 10 \%, V_{\text {DS }}=50 \mathrm{mV}(\mathrm{A}), 1,5 \mathrm{~V}(\mathrm{~B})$ e $3 \mathrm{~V}(\mathrm{C})$ e $\mathrm{L}_{\mathrm{LD}} / \mathrm{L}$ de $0.2,0.3$, $0.4,0.5$.

Figura 42 - Desvio relativo da corrente de dreno em função da sobretensão de condução de $\mathrm{V}_{\mathrm{DS}}=50 \mathrm{mV}(\mathrm{A}), 1,5 \mathrm{~V}(\mathrm{~B})$ e $3 \mathrm{~V}(\mathrm{C})$, com concentração de $5.10^{16} \mathrm{~cm}^{-3}$ variando $\pm 10 \% \ldots . .83$ Figura 43 - Desvio relativo da transcondutância em função da sobretensão de condução de $\mathrm{V}_{\mathrm{DS}}=50 \mathrm{mV}(\mathrm{A}), 1,5 \mathrm{~V}(\mathrm{~B})$ e $3 \mathrm{~V}(\mathrm{C})$, com concentração de $5.10^{16} \mathrm{~cm}^{-3}$ variando $\pm 10 \% \ldots . .85$

Figura 44 - Curva IDS em função de $V_{\mathrm{DS}}$ com sobretensão de condução $\mathrm{V}_{\mathrm{GT}}=600 \mathrm{mV}$ com concentração de $5.10^{16} \mathrm{~cm}^{-3}$ variando $\pm 10 \%$.

Figura 45 - Desvio relativo da corrente de dreno em função de $V_{D S}$ com sobretensão de condução $\mathrm{V}_{\mathrm{GT}}=0 \mathrm{mV}$ (A), $200 \mathrm{mV}$ (B), $400 \mathrm{mV}$ (C) e $600 \mathrm{mV}$ (D), com concentração de $5.10^{16} \mathrm{~cm}^{-3}$ variando $\pm 10 \%$

Figura 46 - Tensão Early em função da tensão de dreno com sobretensão de condução $\mathrm{V}_{\mathrm{GT}}$ $=200 \mathrm{mV}$ com concentração de $5 \cdot 10^{16} \mathrm{~cm}^{-3}$ variando $\pm 10 \%$. 


\section{LISTA DE TABELAS}

Tabela 1 - Tensão Early extraída através de curvas $\mathrm{I}_{\mathrm{DS}}$ em função de $\mathrm{V}_{\mathrm{DS}}$ experimentais...... 46

Tabela 2 - Tensão de ruptura extraída de curvas IDS x $V_{D S}$ experimentais............................. 46

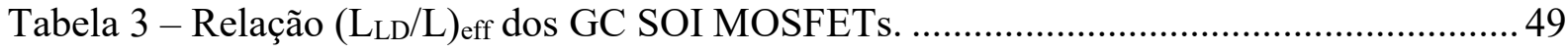

Tabela 4 - Inclinação de Sublimiar (S) dos SOI MOSFETs uniformemente dopado e GC

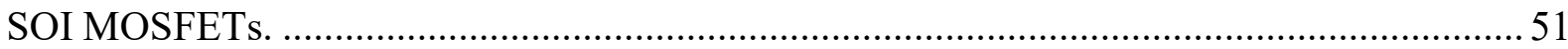

Tabela 5 - Valor médio e desvio padrão relativo da tensão de limiar de transistores SOI MOSFETs uniformemente dopado e GC SOI MOSFETs..................................................... 51

Tabela 6 - Valor médio e desvio relativo da transcondutância média para GC SOI MOSFETs, extraído para $\mathrm{V}_{\mathrm{GT}}=200 \mathrm{mV}$ e $\mathrm{V}_{\mathrm{DS}}=1,5 \mathrm{~V}$.

Tabela 7 - Valor médio e desvio relativo da condutância de dreno $\left(\mathrm{g}_{\mathrm{D}}\right)$ para GC SOI MOSFETs, extraídos para $\mathrm{V}_{\mathrm{GT}}=200 \mathrm{mV}$ e $\mathrm{V}_{\mathrm{DS}}=1,5 \mathrm{~V}$.

Tabela 8 - Tensão Early (VEA) dos transistores GC SOI MOSFETS e SOI uniformemente dopados, com $\mathrm{V}_{\mathrm{DS}}=1,5 \mathrm{~V}$ e $\mathrm{V}_{\mathrm{GT}}=200 \mathrm{mV}$.

Tabela 9 - Ganho de tensão (Av) dos transistores GC SOI MOSFETS e SOI uniformemente dopados, com $\mathrm{V}_{\mathrm{DS}}=1,5 \mathrm{~V}$ e $\mathrm{V}_{\mathrm{GT}}=200 \mathrm{mV}$.

Tabela 10 - Percentual de variação de $\pm 30 \mathrm{~nm}$ em LHD.

Tabela 11 - Valor médio e desvio relativo da tensão de limiar de GC SOI MOSFETs com $\mathrm{L}_{\mathrm{HD}}$ variando $\pm 30 \mathrm{~nm}, \mathrm{~V}_{\mathrm{DS}}=50 \mathrm{mV}$ e $\mathrm{L}_{\mathrm{LD}} / \mathrm{L}$ de $0.2,0.3,0.4,0.5$.

Tabela 12 - Valor médio e desvio relativo da inclinação de sublimiar de GC SOI MOSFETs com $\mathrm{L}_{\mathrm{HD}}$ variando $\pm 30 \mathrm{~nm}, \mathrm{~V}_{\mathrm{DS}}=50 \mathrm{mV}$ e $\mathrm{L}_{\mathrm{LD}} / \mathrm{L}$ de $0.2,0.3,0.4,0.5$.

Tabela 13 - Valor médio e desvio relativo da transcondutância para GC SOI MOSFETs, extraído para $\mathrm{V}_{\mathrm{GT}}=200 \mathrm{mV}$ e $\mathrm{V}_{\mathrm{DS}}=1,5 \mathrm{~V}$.

Tabela 14 - Valor médio e desvio relativo da condutância de dreno para GC SOI MOSFETs, extraídos para $\mathrm{V}_{\mathrm{GT}}=200 \mathrm{mV}$ e $\mathrm{V}_{\mathrm{DS}}=1,5 \mathrm{~V}$.

Tabela 15 - Tensão Early e desvio relativo obtidas a partir das simulações dos GC SOI MOSFETs com $\mathrm{V}_{\mathrm{GT}}=200 \mathrm{mV}$ e $\mathrm{V}_{\mathrm{DS}}=1,5 \mathrm{~V}$ com $\mathrm{L}_{\mathrm{HD}}$ variando $\pm 30 \mathrm{~nm}$ .78

Tabela 16 - Ganho de tensão e desvio relativo dos GC SOI MOSFETs com $V_{\mathrm{GT}}=200 \mathrm{mV}$ e $\mathrm{V}_{\mathrm{DS}}=1,5 \mathrm{~V}$ com $\mathrm{L}_{\mathrm{HD}}$ variando $\pm 30 \mathrm{~nm}$.

Tabela 17 - Valor médio e desvio relativo da tensão de limiar de GC SOI MOSFETs com concentração de $5.10^{16} \mathrm{~cm}^{-3}$ variando $\pm 10 \%, \mathrm{~V}_{\mathrm{DS}}=50 \mathrm{mV}$ e $\mathrm{L}_{\mathrm{LD}} / \mathrm{L}$ de $0.2,0.3,0.4,0.5 \ldots . .82$ 
Tabela 18 - Valor médio e desvio relativo da inclinação de sublimiar de GC SOI MOSFETs com concentração de $5.10^{16} \mathrm{~cm}^{-3}$ variando $\pm 10 \%, \mathrm{~V}_{\mathrm{DS}}=50 \mathrm{mV}$ e $\mathrm{L}_{\mathrm{LD}} / \mathrm{L}$ de 0.2 , $0.3,0.4,0.5$

Tabela 19 - Valor médio e desvio relativo da transcondutância para GC SOI MOSFETs, extraído para $\mathrm{V}_{\mathrm{GT}}=200 \mathrm{mV}$ e $\mathrm{V}_{\mathrm{DS}}=1,5 \mathrm{~V}$ com concentração de $5.10^{16} \mathrm{~cm}^{-3}$ variando \pm $10 \%$

Tabela 20 - Valor médio e desvio relativo da condutância de dreno para GC SOI MOSFETs, extraídos para $\mathrm{V}_{\mathrm{GT}}=200 \mathrm{mV}$ e $\mathrm{V}_{\mathrm{DS}}=1,5 \mathrm{~V}$ com concentração de $5.10^{16} \mathrm{~cm}^{-3}$ variando $\pm 10 \%$

Tabela 21 - Tensão Early e desvio relativo dos GC SOI MOSFETs com $\mathrm{V}_{\mathrm{GT}}=200 \mathrm{mV}$ e $\mathrm{V}_{\mathrm{DS}}=1,5 \mathrm{~V}$ com concentração de $5.10^{16} \mathrm{~cm}^{-3}$ variando $\pm 10 \%$.

Tabela 22 - Ganho de tensão e desvio relativo dos GC SOI MOSFETs com $\mathrm{V}_{\mathrm{GT}}=200 \mathrm{mV}$ e $\mathrm{V}_{\mathrm{DS}}=1,5 \mathrm{~V}$ concentração de $5.10^{16} \mathrm{~cm}^{-3}$ variando $\pm 10 \%$ 


\section{LISTA DE ABREVIATURAS E SIGLAS}

$\begin{array}{ll}\text { CMOS } & \text { Complementary Metal-Oxide-Semiconductor } \\ \text { FD } & \text { Fully Depleted } \\ \text { GC } & \text { Graded-Channel } \\ \text { HD } & \text { Highly Doped } \\ \text { LD } & \text { Lightly Doped } \\ \text { LDD } & \text { Lightly Doped Drain } \\ \text { MOSFET } & \text { Metal-Oxide-Semiconductor Field-Effect Transistor } \\ \text { NFD } & \text { Near-Fully Depleted } \\ \text { PD } & \text { Partially Depleted } \\ \text { SOI } & \text { Silicon-On-Insular }\end{array}$




\section{LISTA DE SÍMBOLOS}

Av Ganho de tensão de malha aberta em baixa frequência [dB]

$\mathrm{BV}_{\mathrm{DS}} \quad$ Tensão de ruptura de dreno [V]

$\mathrm{C}_{\mathrm{D}}$

Capacitância da região de depleção por unidade de área $\left[\mathrm{F} / \mathrm{cm}^{2}\right]$

$\mathrm{C}_{\mathrm{L}}$

Capacitância de carga $[\mathrm{F}]$

$\mathrm{C}_{\mathrm{ox}}$

Capacitância do óxido de porta do transistor MOS por unidade de área $\left[\mathrm{F} / \mathrm{cm}^{2}\right]$

$\mathrm{C}_{\mathrm{oxb}}$

Capacitância do óxido enterrado por unidade de área $\left[\mathrm{F} / \mathrm{cm}^{2}\right]$

$\mathrm{C}_{\text {oxf }}$

Capacitância do óxido de porta do SOI MOSFET por unidade de área $\left[\mathrm{F} / \mathrm{cm}^{2}\right]$

$\mathrm{C}_{\mathrm{Si}}$

Capacitância da camada de silício por unidade de área $\left[\mathrm{F} / \mathrm{cm}^{2}\right]$

E

Campo elétrico $[\mathrm{V} / \mathrm{cm}]$

$\mathrm{E}_{\mathrm{C}}$

Nível de energia inferior da faixa de condução [eV]

$\mathrm{E}_{\mathrm{F}}$

Nível de Fermi do semicondutor [eV]

$\mathrm{E}_{\mathrm{FM}}$

Nível de Fermi do metal [eV]

$\mathrm{E}_{\mathrm{i}}$

Nível de Fermi intrínseco $[\mathrm{eV}]$

$E_{\text {eff }}$

Campo elétrico normal médio no canal nas direções vertical e lateral [V/cm]

$\mathrm{E}_{\mathrm{SF}}$

Campo elétrico abaixo do óxido de porta do transistor SOI [V/cm]

$\mathrm{E}_{\mathrm{V}}$

Nível de energia superior da faixa de valência $[\mathrm{eV}]$

$\mathrm{g}_{\mathrm{D}}$

Condutância de dreno [S]

gDsat

Condutância de dreno na região de saturação [S]

$\mathrm{g}_{\mathrm{m}}$

Transcondutância do transistor [S]

$\mathrm{g}_{\mathrm{m}} / \mathrm{I}_{\mathrm{DS}}$

Relação entre a transcondutância e a corrente de dreno do transistor MOS [V $\left.{ }^{-1}\right]$

Ibias

Corrente constante de polarização fornecida pela fonte de corrente [A]

IDS

Corrente entre dreno e fonte $[\mathrm{A}]$

IDSsat

Corrente de saturação entre dreno e fonte $[\mathrm{A}]$

$\mathrm{k}$

Constante de Boltzmann $\left[1,38066 \times 10^{-23} \mathrm{~J} / \mathrm{K}\right]$

$\mathrm{L}$

Comprimento de máscara do canal do transistor $[\mu \mathrm{m}]$

Leff

Comprimento efetivo do canal do transistor $[\mu \mathrm{m}]$

$\mathrm{L}_{\mathrm{HD}}$

Comprimento da região fortemente dopada no transistor GC SOI $[\mu \mathrm{m}]$

$\mathrm{L}_{\mathrm{LD}}$

Comprimento da região fracamente dopada no transistor GC SOI $[\mu \mathrm{m}]$

$\mathrm{LLD}_{\mathrm{LD}} / \mathrm{L}$

Razão entre os comprimentos das regiões fracamente dopada e o comprimento de canal, no transistor GC SOI 


\begin{tabular}{|c|c|}
\hline $\mathrm{N}_{\mathrm{A}}$ & Concentração de impurezas aceitadoras em um semicondutor $\left[\mathrm{cm}^{-3}\right]$ \\
\hline $\mathrm{N}_{\mathrm{AH}}$ & $\begin{array}{l}\text { Concentração de dopantes da camada de silício do transistor GC SOI na região } \\
\text { fortemente dopada do canal }\left[\mathrm{cm}^{-3}\right]\end{array}$ \\
\hline $\mathrm{N}_{\mathrm{AL}}$ & $\begin{array}{l}\text { Concentração de dopantes da camada de silício do transistor GC SOI na região } \\
\text { fracamente dopada do canal }\left[\mathrm{cm}^{-3}\right]\end{array}$ \\
\hline $\mathrm{N}_{\mathrm{A}, \mathrm{eff}}$ & Concentração efetiva de impurezas aceitadoras em um semicondutor $\left[\mathrm{cm}^{-3}\right]$ \\
\hline$n_{i}$ & Concentração intrínseca de portadores $\left[\mathrm{cm}^{-3}\right]$ \\
\hline q & Carga elementar do elétron $\left[1,6 \times 10^{-19} \mathrm{C}\right]$ \\
\hline$Q_{D}$ & $\begin{array}{l}\text { Densidade de carga de inversão na fronteira do canal com a região de dreno do } \\
\text { transistor SOI }\left[\mathrm{C} / \mathrm{cm}^{2}\right]\end{array}$ \\
\hline$Q_{\text {depl }}$ & Carga de depleção na camada de silício $\left[\mathrm{C} / \mathrm{cm}^{2}\right]$ \\
\hline$Q_{\text {depl,ef }}$ & Carga de depleção efetiva da camada de silício $\left[\mathrm{C} / \mathrm{cm}^{2}\right]$ \\
\hline Qinvf $_{\text {in }}$ & Densidade de carga de inversão na primeira interface do transistor $\left[\mathrm{C} / \mathrm{cm}^{2}\right]$ \\
\hline Qox & Densidade de cargas fixas no óxido de porta do transistor MOS $\left[\mathrm{C} / \mathrm{cm}^{2}\right]$ \\
\hline Qoxb & Densidade de cargas fixas no óxido enterrado $\left[\mathrm{C} / \mathrm{cm}^{2}\right]$ \\
\hline Qoxf & Densidade de cargas fixas no óxido de porta na primeira interface $\left[\mathrm{C} / \mathrm{cm}^{2}\right]$ \\
\hline Qs & $\begin{array}{l}\text { Densidade de carga de inversão na fronteira do canal com a região de fonte do } \\
\text { transistor SOI }\left[\mathrm{C} / \mathrm{cm}^{2}\right]\end{array}$ \\
\hline $\mathrm{QSB}_{\mathrm{SB}}$ & $\begin{array}{l}\text { Carga de acumulação }\left(\mathrm{Q}_{\mathrm{SB}}>0\right) \text { ou inversão }\left(\mathrm{Q}_{\mathrm{SB}}<0\right) \text { na segunda interface } \\
{\left[\mathrm{C} / \mathrm{cm}^{2}\right]}\end{array}$ \\
\hline $\mathrm{S}$ & Inclinação de sublimiar $[\mathrm{mV} / \mathrm{dec}]$ \\
\hline $\mathrm{T}$ & Temperatura absoluta $[\mathrm{K}]$ \\
\hline$t_{\mathrm{oxb}}$ & Espessura do óxido enterrado [nm] \\
\hline$t_{\text {oxf }}$ & Espessura do óxido de porta $[\mathrm{nm}]$ \\
\hline $\mathrm{t}_{\mathrm{Si}}$ & Espessura da camada de silício [nm] \\
\hline $\mathrm{V}_{\mathrm{D}}$ & Tensão aplicada ao dreno do transistor SOI [V] \\
\hline $\mathrm{V}_{\mathrm{DE}}$ & Tensão efetiva no canal no fim do dreno [V] \\
\hline $\mathrm{v}_{\mathrm{ds}}$ & $\begin{array}{l}\text { Componente alternada da tensão de saída do amplificador operacional de } \\
\text { transcondutância [V] }\end{array}$ \\
\hline $\mathrm{V}_{\mathrm{DS}}$ & Tensão entre dreno e fonte [V] \\
\hline $\mathrm{V}_{\mathrm{EA}}$ & Tensão Early [V] \\
\hline $\mathrm{V}_{\mathrm{FB}}$ & Tensão de faixa plana da estrutura MOS [V] \\
\hline $\mathrm{V}_{\mathrm{GB}}$ & Tensão aplicada ao substrato do transistor SOI [V] \\
\hline $\mathrm{V}_{\mathrm{GB}, \mathrm{acc}}$ & Tensão aplicada ao substrato para que a segunda interface acumule [V] \\
\hline
\end{tabular}




\begin{tabular}{|c|c|}
\hline $\mathrm{Vgf}$ & $\begin{array}{l}\text { Componente alternada da tensão de entrada do amplificador operacional de } \\
\text { transcondutância [V] }\end{array}$ \\
\hline $\mathrm{V}_{\mathrm{GF}}$ & Tensão aplicada à primeira porta do transistor SOI [V] \\
\hline $\mathrm{V}_{\mathrm{GT}}$ & Sobretensão de condução [V] \\
\hline $\mathrm{V}_{\text {IN }}$ & Tensão de entrada do amplificador operacional de transcondutância [V] \\
\hline Vout & Tensão de saída do amplificador operacional de transcondutância [V] \\
\hline $\mathrm{V}_{\mathrm{S}}$ & Tensão aplicada à fonte do transistor SOI [V] \\
\hline $\mathrm{V}_{\mathrm{thf}}$ & Tensão de limiar da primeira interface em inversão forte [V] \\
\hline $\mathrm{V}_{\text {thf,acc }}$ & Tensão de limiar da primeira interface com a segunda interface acumulada [V] \\
\hline $\mathrm{V}_{\text {thf,depl }}$ & Tensão de limiar da primeira interface com a segunda interface depletada [V] \\
\hline $\mathrm{V}_{\text {thf,inv }}$ & Tensão de limiar da primeira interface com a segunda interface invertida [V] \\
\hline $\mathrm{V}_{\text {thf,mean }}$ & Tensão de limiar média [V] \\
\hline W & Largura do canal do transistor $[\mu \mathrm{m}]$ \\
\hline $\mathrm{x}$ & Eixo na direção da profundidade da camada de silício \\
\hline $\mathrm{X}_{\mathrm{dmax}}$ & Profundidade máxima da região de depleção [ $\mu \mathrm{m}]$ \\
\hline$\alpha$ & Parâmetro resultante da associação das capacitâncias do transistor MOS \\
\hline$\alpha_{1}$ & Parâmetro de ajuste \\
\hline$\alpha_{2}$ & Parâmetro de ajuste \\
\hline$\alpha_{3}$ & Parâmetro de ajuste \\
\hline$\alpha_{\mathrm{S}}$ & Constante de espalhamento $[\mathrm{cm} / \mathrm{V}]$ \\
\hline$\beta$ & Fator de corrente \\
\hline$\varepsilon_{0}$ & Permissividade do vácuo $\left[8,85 \times 10^{-12} \mathrm{~F} / \mathrm{cm}\right]$ \\
\hline$\varepsilon_{\mathrm{ox}}$ & Permissividade do óxido de silício $\left[3,45 \times 10^{-13} \mathrm{~F} / \mathrm{cm}\right]$ \\
\hline$\varepsilon_{\mathrm{Si}}$ & Permissividade do silício $\left[1,06 \times 10^{-12} \mathrm{~F} / \mathrm{cm}\right]$ \\
\hline$\Delta \mathrm{I}_{\mathrm{DS}} / \mathrm{I}_{\mathrm{DS}}$ & Desvio relativo na corrente de dreno \\
\hline$\Delta \mathrm{V}_{\text {thf }} / \mathrm{V}_{\text {thf }}$ & Desvio relativo na tensão de limiar \\
\hline$\Delta \beta / \beta$ & Desvio relativo no fator de corrente \\
\hline$\Phi$ & Potencial $[\mathrm{V}]$ \\
\hline$\Phi_{\mathrm{F}}$ & Potencial de Fermi $[\mathrm{V}]$ \\
\hline$\Phi_{\mathrm{MS}}$ & Diferença da função de trabalho entre metal e silício [V] \\
\hline$\Phi_{\mathrm{MSB}}$ & Diferença de função de trabalho entre o substrato e a camada de silício [V] \\
\hline$\Phi_{\mathrm{MSF}}$ & Diferença da função de trabalho entre metal de porta e semicondutor [V] \\
\hline
\end{tabular}


$\Phi_{\text {oxf }}$

$\Phi_{\text {oxb }}$

$\Phi_{\mathrm{SB}}$

$\Phi_{\mathrm{SF}}$

$\lambda$

$\mu_{n}$

$\mu_{0}$

$\sigma \mathrm{A}$

$\sigma \mathrm{g}_{\mathrm{D}}$

$\sigma g_{m}$

$\sigma \mathrm{V}_{\text {thf }}$

$\sigma \mathrm{V}_{\text {thf,CED }}$

$\sigma_{0} \mathrm{~V}_{\text {thf }}$
Queda de potencial no óxido de porta do transistor SOI [V]

Queda de potencial no óxido enterrado do transistor SOI [V]

Potencial de superfície da segunda interface do transistor SOI [V]

Potencial de superfície da primeira interface do transistor SOI [V]

Parâmetro que considera o efeito de modulação do comprimento de canal devido à tensão aplicada ao dreno $\left[\mathrm{V}^{-1}\right]$

Mobilidade efetiva dos elétrons na região do canal $\left[\mathrm{cm}^{2} / \mathrm{V} . \mathrm{s}\right]$

Mobilidade dos portadores independente de campo elétrico

Desvio padrão do ganho de tensão [V/V]

Desvio padrão da condutância de dreno [S]

Desvio padrão da transcondutância [S]

Desvio padrão da tensão de limiar [V]

Desvio padrão da tensão de limiar em relação à concentração efetiva de dopantes [V]

Desvio padrão da tensão de limiar normalizado em relação à área de porta [V] 
1 INTRODUÇÃO

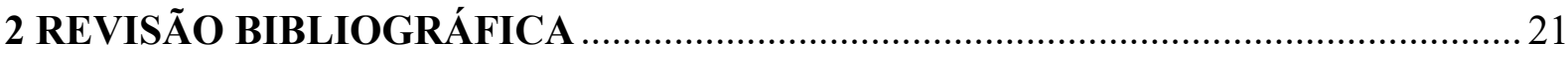

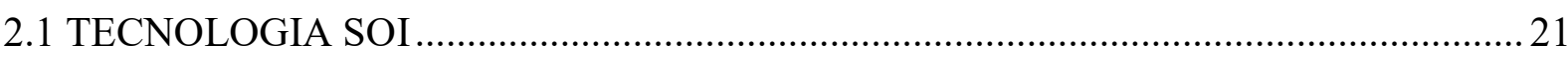

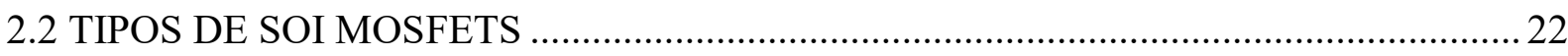

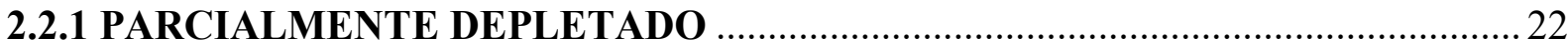

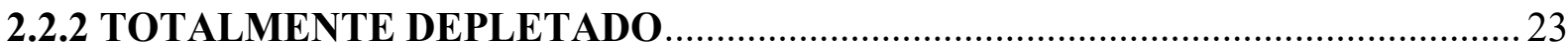

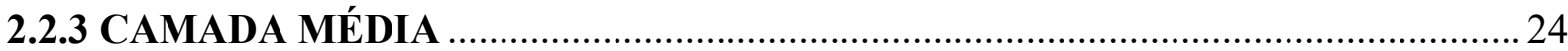

2.3 VANTAGENS DOS SOI MOSFETS TOTALMENTE DEPLETADOS (FD SOI ) ........ 25

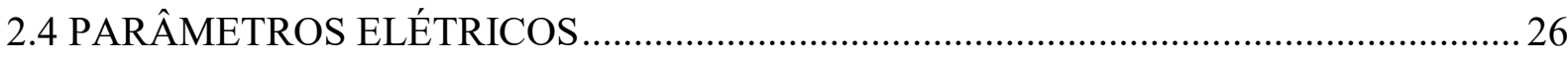

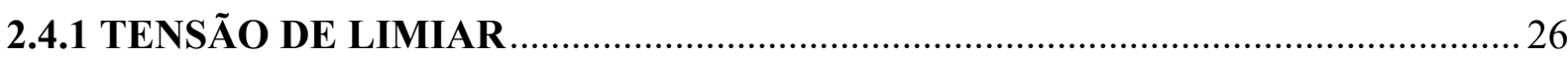

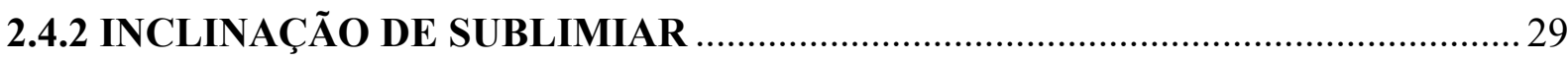

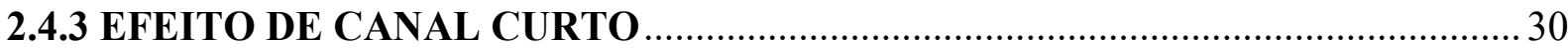

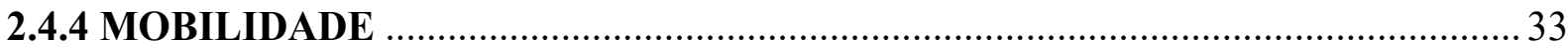

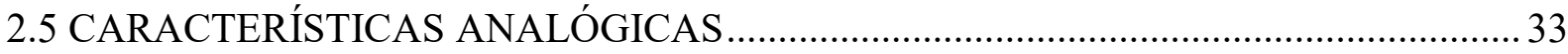

2.5.1 TRANSCONDUTÂNCIA E RAZÃO g $_{\mathrm{m}} / \mathbf{I}$

2.5.2 CONDUTÂNCIA DE DRENO E TENSÃO EARLY ............................................ 37

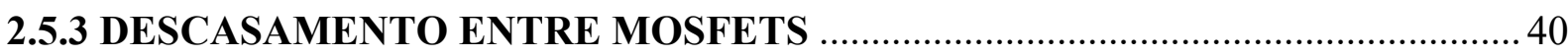

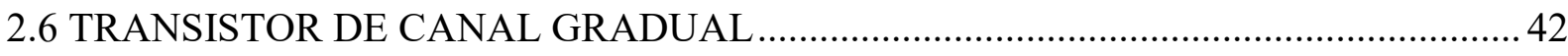

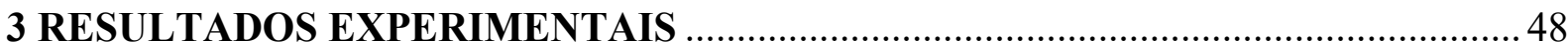

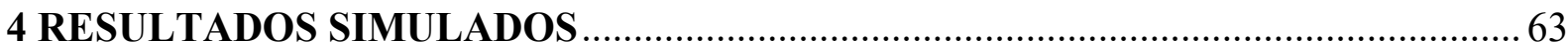

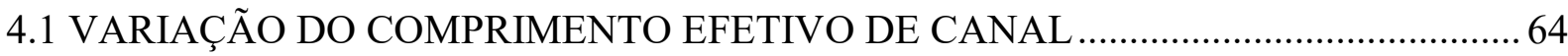

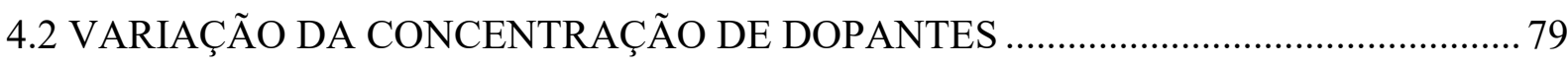

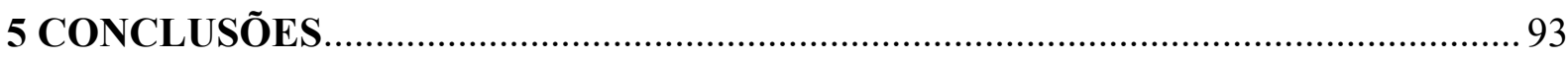

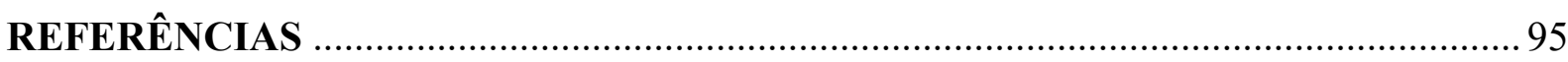

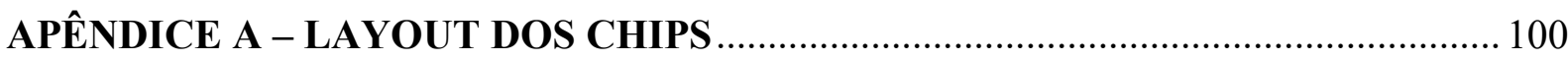

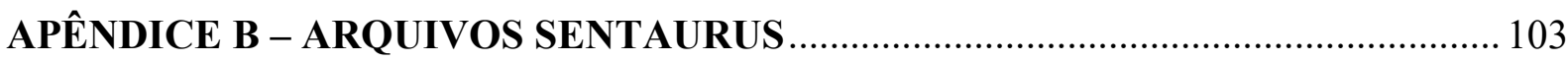

APÊNDICE C - TRABALHOS PUBLICADOS ........................................................ 113 


\section{INTRODUÇÃO}

Com a constante necessidade de produção de dispositivos cada vez menores usando-se lâminas de melhor qualidade e menor custo, uma nova tecnologia começou a ser estudada. A tecnologia Silício-Sobre-Isolante (SOI) MOSFETs apresenta dispositivos fabricados sobre uma camada fina de silício, separada do restante do substrato por um material isolante. Inicialmente, devido à dificuldade de obtenção de lâminas de camada fina com espessura uniforme, eram produzidos dispositivos de camada espessa que operavam parcialmente depletados. A redução da espessura da camada de silício deu origem aos chamados dispositivos totalmente depletados (COLINGE, 2004). Estes dispositivos apresentam vantagens em relação aos dispositivos SOI anteriores, como redução do efeito de canal curto (YOUNG, 1989), elevação da tensão de ruptura, aumento da transcondutância (COLINGE, 2004), aumento da mobilidade dos portadores (YOSHIMI et al., 1988), entre outros.

Apesar de apresentar vantagens em relação aos MOSFETs implementados em lâminas de silício convencionais (Bulk), os transistores SOI apresentam baixa tensão de ruptura, devido à presença do corpo flutuante, o qual ativa o transistor bipolar parasitário, formado pelas regiões de fonte, canal e dreno (CHOI, 1991).

Com o intuito de reduzir a ocorrência de efeito bipolar parasitário e aumentar a tensão de ruptura em SOI MOSFETs de camada fina, foi desenvolvida uma nova estrutura para o SOI MOSFET, com dopagem assimétrica na região de canal, chamado de transistor de Canal Gradual (GC SOI). A região de canal próximo à fonte é fortemente dopada, o que permite fixar a tensão de limiar do dispositivo, e fracamente dopada na região de canal perto ao dreno com o intuito de diminuir campo elétrico vertical na junção canal-dreno, e, por consequência, o efeito de ionização por impacto. Os resultados obtidos foram bastante promissores, com elevação da tensão de ruptura, diminuição da condutância do dreno, aumento da tensão Early, aumento da corrente de saturação e da transcondutância (COLINGE, 2004).

$\mathrm{O}$ descasamento elétrico refere-se às pequenas variações de desempenho elétrico de dispositivos projetados com dimensões idênticas e implementados pelo mesmo processo de fabricação. O casamento das características elétricas de transistores é extremamente importante para circuitos analógicos, uma vez que diversos circuitos integrados são projetados com base em características semelhantes para dispositivos idênticos, tais como amplificadores operacionais de transcondutância (OTA) (PAVANELLO et al., 2000a). Apesar das diversas vantagens reportadas para os GC SOI MOSFETs para aplicações analógicas, pouca informação está disponível a respeito do descasamento de suas características elétricas. 
Este trabalho tem como objetivo o estudo do descasamento de SOI MOSFETs de canal gradual operando em saturação, através de resultados de simulações numéricas bidimensionais e medidas experimentais em comparação com dispositivos uniformemente dopados com mesmas dimensões. 


\section{REVISÃO BIBLIOGRÁFICA}

Neste capítulo serão apresentados os tipos de SOI MOSFETs, suas características elétricas básicas e analógicas, dispositivos GC SOI MOSFET e suas vantagens em relação aos transistores SOI convencionais. Será apresentada ainda uma revisão sobre descasamento de características elétricas em transistores MOS.

\subsection{TECNOLOGIA SOI}

Os transistores MOS convencionais são produzidos em lâminas de silício, com espessura de aproximadamente $500 \mu \mathrm{m}$, onde somente a parte superficial desta lâmina, uma fração do primeiro micrometro, é utilizada efetivamente para a fabricação dos dispositivos. No substrato restante há a presença de efeitos parasitários indesejados devido à interação com a parte ativa, como o aumento da capacitância parasitária e efeito do tiristor parasitário (COLINGE, 2004; PAVANELLO, 2000).

A demanda atual de transistores com comprimento de canal efetivo cada vez menor faz com que esses efeitos indesejados se tornem mais pronunciados, como o efeito tiristor parasitário e a elevação das capacitâncias parasitárias (COLINGE, 2004), fazendo com que haja um acréscimo das etapas de produção para tentar minimizar esses efeitos, acarretando um aumento do custo de produção.

Na tecnologia SOI MOSFET há a separação da região ativa da lâmina, onde os dispositivos são fabricados, do substrato remanescente, através da presença de um material isolante chamado de óxido enterrado. Esse material isolante pode variar, sendo o mais comum o dióxido de silício $\left(\mathrm{SiO}_{2}\right)$ (COLINGE, 2004). A presença desse óxido enterrado reduz os efeitos parasitários, além de promover uma redução no número de etapas de fabricação devido à ausência das cavidades (COLINGE, 2004).

A Figura 1 apresenta o perfil transversal de um transistor SOI nMOSFET, indicando os eletrodos de porta $\left(\mathrm{V}_{\mathrm{GF}}\right)$, fonte $\left(\mathrm{V}_{\mathrm{S}}\right)$, dreno $\left(\mathrm{V}_{\mathrm{D}}\right)$ e substrato $\left(\mathrm{V}_{\mathrm{GB}}\right)$, as espessuras de óxido de porta $\left(\mathrm{t}_{\mathrm{oxf}}\right)$, camada de silício $\left(\mathrm{t}_{\mathrm{Si}}\right)$ e óxido enterrado $\left(\mathrm{t}_{\mathrm{oxb}}\right)$, bem como as três interfaces Si$\mathrm{SiO}_{2}$ existentes na estrutura. 
Figura 1 - Perfil transversal de um SOI nMOSFET.

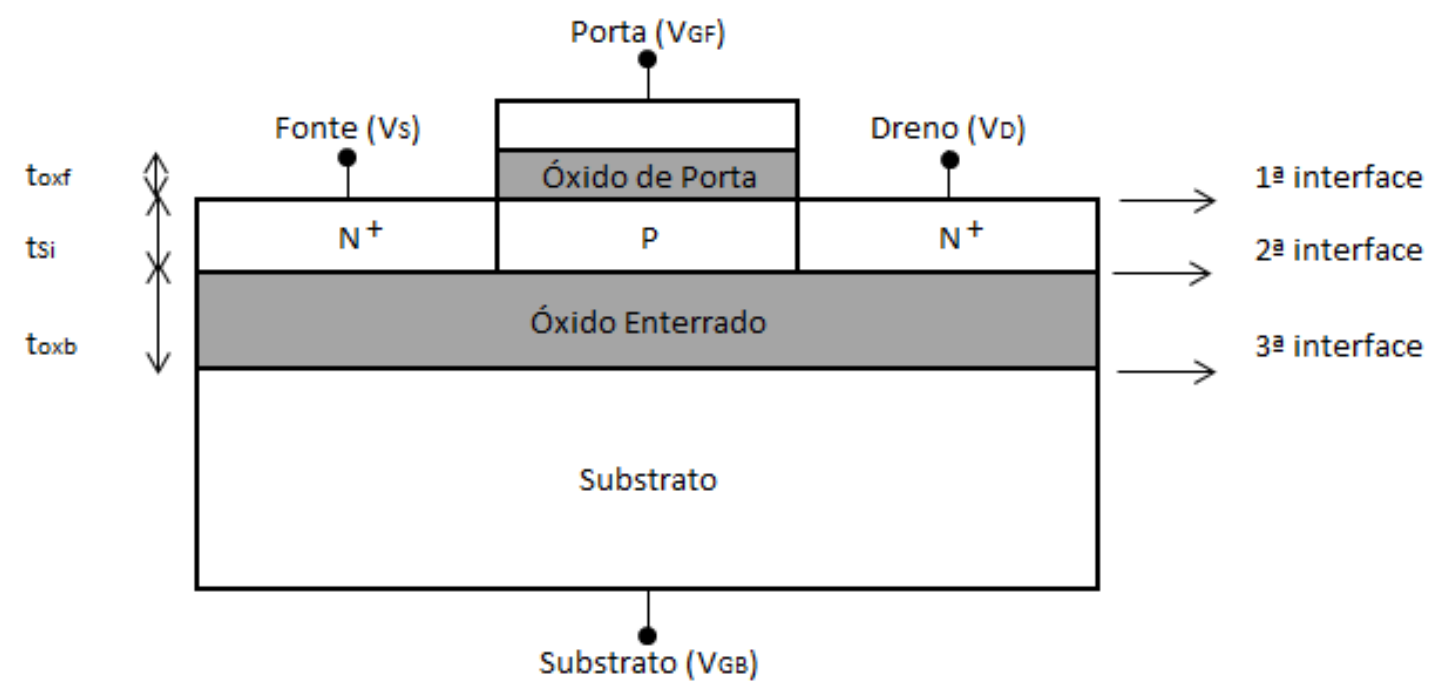

Fonte: Adaptado de Souza, 2008.

\subsection{TIPOS DE SOI MOSFETS}

O modo de funcionamento do SOI MOSFET está relacionado com a espessura da camada de silício, a concentração de dopantes e a temperatura de operação.

A região de depleção estende-se da região da primeira interface $\mathrm{Si}_{-} \mathrm{SiO}_{2}$ até sua profundidade máxima, dada por (SZE, 1981):

$$
\mathrm{x}_{\mathrm{dmax}}=\sqrt{\frac{4 \cdot \varepsilon_{\mathrm{Si}} \cdot \Phi_{\mathrm{F}}}{\mathrm{q} \cdot \mathrm{N}_{\mathrm{A}}}}
$$

onde $\varepsilon_{\mathrm{Si}}$ é a permissividade do silício, q a carga elementar do elétron, $\mathrm{N}_{\mathrm{A}}$ a concentração de dopantes e $\Phi_{\mathrm{F}}$ o potencial de Fermi, dado por $\Phi_{\mathrm{F}}=\frac{\mathrm{k} \cdot \mathrm{T}}{\mathrm{q}} \ln \left(\frac{\mathrm{N}_{\mathrm{A}}}{\mathrm{n}_{\mathrm{i}}}\right)$ onde k é a constante de Boltzmann, $\mathrm{T}$ a temperatura absoluta e $\mathrm{n}_{\mathrm{i}}$ a concentração intrínseca de portadores.

\subsubsection{PARCIALMENTE DEPLETADO}

Caso a espessura da camada de silício seja maior que 2. $\mathrm{x}_{\mathrm{dmax}}\left(\mathrm{t}_{\mathrm{Si}}>2 . \mathrm{x}_{\mathrm{dmax}}\right)$ não haverá interação entre as regiões de depleção da primeira e segunda interfaces, fazendo com que os potenciais de superfícies da primeira $\left(\Phi_{\mathrm{SF}}\right)$ e segunda $\left(\Phi_{\mathrm{SB}}\right)$ interfaces sejam independentes. Neste caso o dispositivo é chamado de camada espessa ou parcialmente depletado (PD). 
Caso haja contato de corpo e este seja aterrado, seu funcionamento será similar ao MOS convencional. Caso não haja contato de corpo ou este seja deixado eletricamente flutuando, o transistor apresentará efeitos de corpo flutuante (elevação abrupta de corrente efeito Kink, efeito do transistor bipolar parasitário entre fonte e dreno) (COLINGE, 2004; KRISHNAN, 1998).

A Figura 2 apresenta o diagrama de faixas de energia do transistor SOI de camada espessa. Nesta figura $E_{C}$ é o nível energético mínimo da faixa de condução, $E_{V}$ o nível energético máximo da camada de valência, $\mathrm{E}_{\mathrm{i}} \mathrm{o}$ nível intrínseco, $\mathrm{E}_{\mathrm{F}} \mathrm{O}$ nível de Fermi e $\mathrm{E}_{\mathrm{FM}} \mathrm{o}$ nível de Fermi do eletrodo de porta.

Figura 2 - Diagrama de faixas de energia do SOI MOSFET de camada espessa.

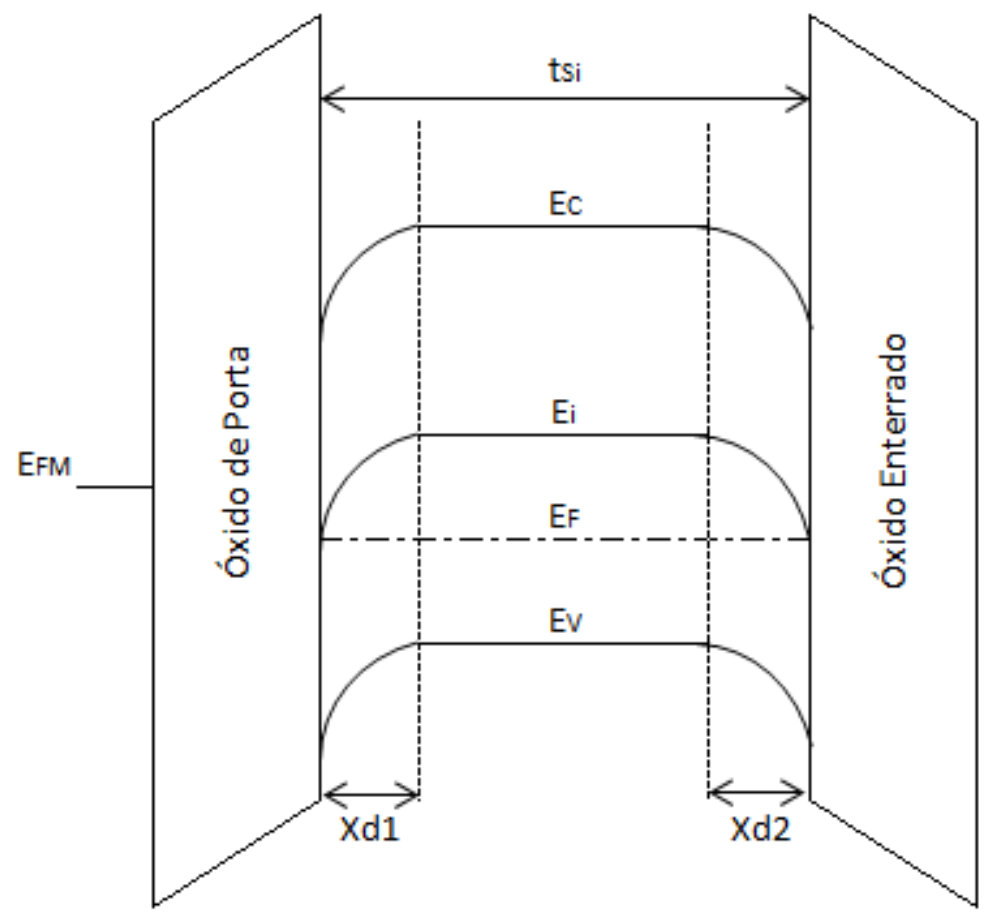

Fonte: Adaptado de Pavanello, 2000.

\subsubsection{TOTALMENTE DEPLETADO}

Caso a espessura da camada de silício seja menor que $\mathrm{x}_{\mathrm{dmax}}\left(\mathrm{t}_{\mathrm{Si}}<\mathrm{x}_{\mathrm{dmax}}\right)$ haverá interação entre as regiões de depleção da primeira e segunda interfaces quando houver aplicação de uma tensão de porta maior que a tensão de limiar, fazendo com que os potenciais de superfícies da primeira e segunda interfaces sejam relacionados, gerando um acoplamento elétrico. Neste caso o dispositivo é chamado de camada fina ou totalmente depletado (FD). 
Estes dispositivos apresentam as melhores características elétricas entre os dispositivos SOI, que será discutido futuramente, porém apresenta uma dependência da tensão de limiar com a espessura da camada de silício (CHUANG et al., 1998), o que é indesejado na operação de circuitos integrados.

A Figura 3 apresenta o diagrama de faixas de energia do transistor SOI de camada fina.

Figura 3 - Diagrama de faixas de energia do SOI MOSFET de camada fina.

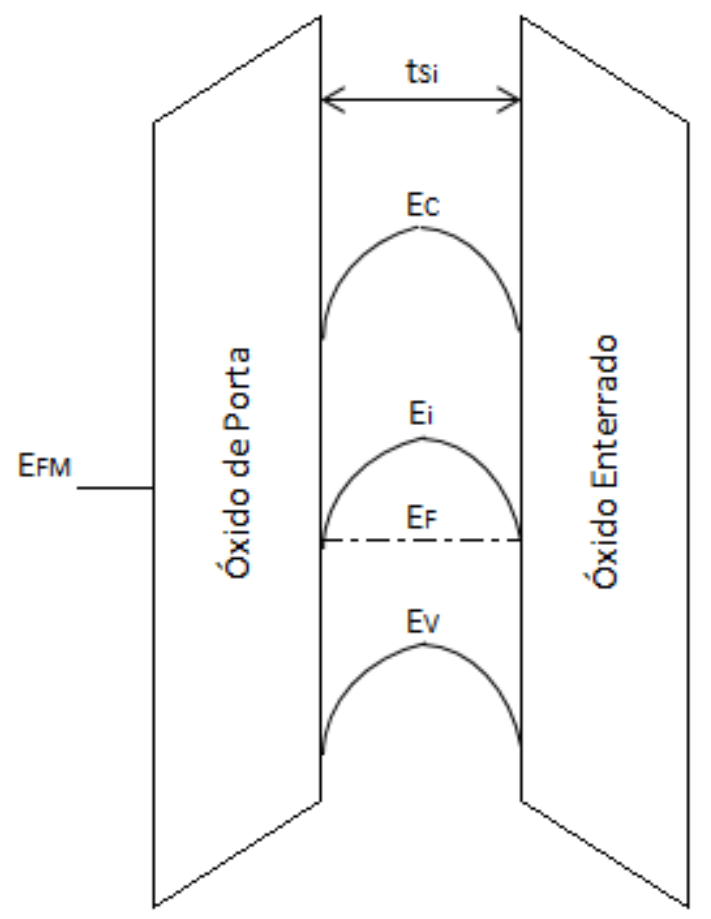

Fonte: Adaptado de Pavanello, 2000.

\subsubsection{CAMADA MÉDIA}

Caso a espessura da camada de silício esteja entre $\mathrm{x}_{\mathrm{dmax}}$ e $2 \mathrm{x}_{\mathrm{dmax}}\left(\mathrm{x}_{\mathrm{dmax}}<\mathrm{t}_{\mathrm{Si}}<2 \mathrm{x}_{\mathrm{dmax}}\right) \mathrm{a}$ região de depleção da primeira e segunda interfaces podem ou não entrar em contato dependendo da tensão de substrato $\left(\mathrm{V}_{\mathrm{GB}}\right)$ aplicada. Neste caso o dispositivo é chamado de camada média (NFD).

Estes dispositivos podem apresentar o comportamento elétrico de um SOI totalmente depletado ou parcialmente depletado, dependendo das condições de operação (KINGET, 2005). 


\subsection{VANTAGENS DOS SOI MOSFETS TOTALMENTE DEPLETADOS (FD SOI )}

O SOI MOSFET totalmente depletado (FD SOI) apresenta melhores características em relação ao SOI parcialmente depletado e ao MOS convencional, tais como: diminuição do campo elétrico horizontal (KISTLER, 1994), aumento da mobilidade dos portadores na região de canal (YOSHIMI, 1989), diminuição da variação da tensão de limiar com a temperatura e diminuição do efeito de canal curto (GROESENEKEN, 1990).

O efeito de corpo (body factor) é a resistência oferecida pelo dispositivo para que o eletrodo de porta controle o potencial de superfície da interface óxido de porta-camada de silício. Este efeito afeta diretamente a capacidade de fornecimento de corrente de dreno do transistor e a inclinação de sublimiar. Quanto menor esse efeito de corpo mais próxima do ideal será a resposta do dispositivo, fazendo com que a inclinação de sublimiar se aproxime do valor mínimo teórico de $60 \mathrm{mV} /$ década em temperatura ambiente (COLINGE, 2004).

O efeito de corpo (n) é expresso por $n=1+\alpha$ onde $\alpha$ é o fator de acoplamento capacitivo do transistor, dado por:

$$
\begin{aligned}
& \alpha=\frac{\mathrm{C}_{\mathrm{D}}}{\mathrm{C}_{\mathrm{ox}}} \text {, para MOS convencional e transistor SOI parcialmente depletado; } \\
& \alpha=\frac{\mathrm{C}_{\mathrm{Si}}}{\mathrm{C}_{\mathrm{oxf}}} \text {, para transistor SOI totalmente depletado com segunda interface em }
\end{aligned}
$$
acumulação;

$\alpha=\frac{\mathrm{C}_{\mathrm{Si}} \cdot \mathrm{C}_{\mathrm{oxb}}}{\mathrm{C}_{\mathrm{oxf}}\left(\mathrm{C}_{\mathrm{Si}}+\mathrm{C}_{\mathrm{oxb}}\right)}$, para transistor SOI totalmente depletado com segunda interface em depleção.

onde $\mathrm{C}_{\text {oxf }}\left(\mathrm{C}_{\mathrm{ox}}\right.$ para transistor MOS convencional) é a capacitância do óxido de porta, $\mathrm{C}_{\text {oxb }} \mathrm{a}$ capacitância do óxido enterrado, $\mathrm{C}_{\mathrm{Si}}$ a capacitância do silício e $\mathrm{C}_{\mathrm{D}}$ a capacitância da região de depleção, todos por unidade de área e expressos por $\mathrm{C}_{\mathrm{oxf}}=\frac{\varepsilon_{\mathrm{ox}}}{\mathrm{t}_{\mathrm{oxf}}} ; \mathrm{C}_{\mathrm{oxb}}=\frac{\varepsilon_{\mathrm{ox}}}{\mathrm{t}_{\mathrm{oxb}}} ; \mathrm{C}_{\mathrm{Si}}=\frac{\varepsilon_{\mathrm{Si}}}{\mathrm{t}_{\mathrm{Si}}} ; \mathrm{C}_{\mathrm{D}}=$ $\frac{\varepsilon_{\mathrm{Si}}}{\mathrm{X}_{\mathrm{dmax}}}$, onde $\varepsilon_{\mathrm{ox}}$ é a permissividade do óxido.

Observa-se que o efeito de corpo em SOI MOSFETs totalmente depletado é menor que nos MOS convencionais, onde os valores numéricos de $\mathrm{n}$ seguem a ordem (FLANDRE, 1994):

$\mathrm{n}$ SOI totalmente depletado $<\mathrm{n}$ MOS convencional $<\mathrm{n}$ SOI com segunda interface em acumulação 
A consequência da diminuição do efeito de corpo pode ser observada pela maior intensidade de corrente de dreno em relação a dispositivos convencionais (COLINGE, 2004; LIM, 1984), como pode ser observado nas equações de primeira ordem da corrente de dreno:

a) triodo:

$$
\mathrm{I}_{\mathrm{DS}} \cong \frac{\mathrm{W} \cdot \mu_{\mathrm{n}} \cdot \mathrm{C}_{\mathrm{oxf}}}{\mathrm{L}}\left[\left(\mathrm{V}_{\mathrm{GF}}-\mathrm{V}_{\mathrm{thf}}\right) \cdot \mathrm{V}_{\mathrm{DS}}-\mathrm{n} \cdot \frac{\mathrm{V}_{\mathrm{DS}}{ }^{2}}{2}\right]
$$

b) saturação:

$$
\mathrm{I}_{\mathrm{DSsat}} \cong \frac{\mathrm{W} \cdot \mu_{\mathrm{n}} \cdot \mathrm{C}_{\mathrm{oxf}}}{2 \cdot \mathrm{L} \cdot \mathrm{n}}\left(\mathrm{V}_{\mathrm{GF}}-\mathrm{V}_{\mathrm{thf}}\right)^{2}
$$

onde $\mathrm{W}$ é a largura do canal, L o comprimento do canal, $\mu_{\mathrm{n}}$ a mobilidade efetiva dos elétrons do canal, $\mathrm{V}_{\text {thf }}$ a tensão de limiar do transistor, $\mathrm{V}_{\mathrm{DS}}$ a tensão entre dreno e fonte, e $\mathrm{V}_{\mathrm{GF}}$ a tensão aplicada à porta.

A redução de $n$ também leva à diminuição da inclinação de sublimiar, aproximando-a do valor teórico (BREWS, 1979):

$$
\mathrm{S}=\frac{\mathrm{k} \cdot \mathrm{T}}{\mathrm{q}} \cdot \ln (10) \cdot \mathrm{n}
$$

desprezando a influência das armadilhas de interface.

\subsection{PARÂMETROS ELÉTRICOS}

Nessa seção serão apresentados os principais parâmetros elétricos de um SOI nMOSFET de camada fina.

\subsubsection{TENSÃO DE LIMIAR}

A tensão de limiar $\left(\mathrm{V}_{\text {thf }}\right)$ é a tensão aplicada à porta que eleva o potencial da superfície da camada de silício para 2. $\Phi_{\mathrm{F}}$. A tensão de limiar de um MOSFET convencional é dada por (VEERARAGHAVAN, 1988): 


$$
\mathrm{V}_{\mathrm{thf}}=\mathrm{V}_{\mathrm{FB}}+2 \Phi_{\mathrm{F}}+\frac{\mathrm{q} \cdot \mathrm{N}_{\mathrm{A}} \cdot \mathrm{x}_{\mathrm{dmax}}}{\mathrm{C}_{\mathrm{ox}}}
$$

onde $\mathrm{V}_{\mathrm{FB}}$ é a tensão de faixa plana dada por $\mathrm{V}_{\mathrm{FB}}=\emptyset_{\mathrm{MS}}-\frac{\mathrm{Qox}}{\mathrm{Cox}}$, sendo $\Phi_{\mathrm{MS}}$ a diferença da função trabalho entre o eletrodo de porta e o silício, e Qox a densidade de carga fixa no óxido de porta por unidade de área.

Em dispositivos SOI parcialmente depletados não há interação entra as regiões de depleção da primeira e da segunda interfaces, e $V_{\text {thf }}$ na primeira interface é a mesma de um transistor MOS convencional (LIM, 1983).

Em dispositivos SOI totalmente depletados há interação entre a região de depleção da primeira e segunda interface. A equação da tensão de limiar pode ser obtida pela equação de Poisson $\frac{\mathrm{d}^{2} \Phi}{\mathrm{dx}^{2}}=\frac{\mathrm{q} \cdot \mathrm{N}_{\mathrm{A}}}{\varepsilon_{\mathrm{Si}}}$, onde $\mathrm{x}$ é o eixo na direção da profundidade da camada de silício. Considerando as interações do potencial da primeira e segunda interfaces, sendo $\Phi_{\mathrm{SF}} \mathrm{o}$ potencial de superfície na primeira interface e $\Phi_{\mathrm{SB}}$ o potencial de superfície na segunda interface, pode-se determinar o potencial em função da profundidade da camada de silício (LIM, 1983):

$$
\Phi(\mathrm{x})=\frac{\mathrm{q} \cdot \mathrm{N}_{\mathrm{A}}}{2 \cdot \varepsilon_{\mathrm{Si}}} \cdot \mathrm{x}^{2}+\left(\frac{\Phi_{\mathrm{SB}}-\Phi_{\mathrm{SF}}}{\mathrm{t}_{\mathrm{Si}}}-\frac{\mathrm{q} \cdot \mathrm{N}_{\mathrm{A}} \cdot \mathrm{t}_{\mathrm{Si}}}{2 \cdot \varepsilon_{\mathrm{Si}}}\right) \cdot \mathrm{x}+\Phi_{\mathrm{SF}}
$$

O campo elétrico vertical na camada de silício é dado pela derivada do potencial ao longo da profundidade $\mathrm{x}$, dada por:

$$
\mathrm{E}(\mathrm{x})=-\frac{\mathrm{d} \Phi(\mathrm{x})}{\mathrm{dx}}=-\frac{\mathrm{q} \cdot \mathrm{N}_{\mathrm{A}}}{\varepsilon_{\mathrm{Si}}} \cdot \mathrm{x}-\left(\frac{\Phi_{\mathrm{SB}}-\Phi_{\mathrm{SF}}}{\mathrm{t}_{\mathrm{Si}}}-\frac{\mathrm{q} \cdot \mathrm{N}_{\mathrm{A}} \cdot \mathrm{t}_{\mathrm{Si}}}{2 \cdot \varepsilon_{\mathrm{Si}}}\right)
$$

Adotando $x=0$ obtém-se o campo elétrico na primeira interface (SZE, 1981):

$$
\mathrm{E}_{\mathrm{SF}}=\left(\frac{\Phi_{\mathrm{SB}}-\Phi_{\mathrm{SF}}}{\mathrm{t}_{\mathrm{Si}}}+\frac{\mathrm{q} \cdot \mathrm{N}_{\mathrm{A}} \cdot \mathrm{t}_{\mathrm{Si}}}{2 \cdot \varepsilon_{\mathrm{Si}}}\right)
$$

Aplicando o teorema de Gauss na primeira interface, obtém-se a queda de potencial no óxido de porta:

$$
\Phi_{\mathrm{oxf}}=\frac{\varepsilon_{\mathrm{Si}} \cdot \mathrm{E}_{\mathrm{SF}}-\mathrm{Q}_{\mathrm{oxf}}-\mathrm{Q}_{\mathrm{invf}}}{\mathrm{C}_{\mathrm{oxf}}}
$$


Onde Qoxf é a densidade de carga fixa no óxido de porta, $\mathrm{C}_{\text {oxf }}$ a capacitância do óxido de porta e $Q_{\text {invf }}$ a densidade de carga de inversão na primeira interface do transistor; todas por unidade de área.

Através do balanço de energia de um SOI MOSFET totalmente depletado tem-se:

a) na primeira interface:

$$
\mathrm{V}_{\mathrm{GF}}=\Phi_{\mathrm{MSF}}+\Phi_{\mathrm{oxf}}+\Phi_{\mathrm{SF}}
$$

b) na segunda interface:

$$
\mathrm{V}_{\mathrm{GB}}=\Phi_{\mathrm{MSB}}+\Phi_{\mathrm{oxb}}+\Phi_{\mathrm{SB}}
$$

Onde $\Phi_{\mathrm{MSF}}$ é a diferença da função trabalho entre metal de porta e a camada de silício e $\Phi_{\text {MSB }}$ a diferença entre o substrato e a camada de silício do transistor, $\Phi_{\text {oxf }}$ a queda de potencial no óxido de porta do transistor, $\Phi_{\mathrm{oxb}}$ no óxido enterrado no transistor e $\mathrm{V}_{\mathrm{GB}}$ é a tensão de substrato do transistor.

Substituindo as equações 7,8 e 9 em 10 e 11 pode-se obter as equações que relacionam as tensões aplicadas na porta e no substrato com os potenciais de superfície da primeira e segunda interface (LIM, 1983).

$$
\begin{aligned}
& \mathrm{V}_{\mathrm{GF}}=\Phi_{\mathrm{MSF}}-\frac{\mathrm{Q}_{\mathrm{oxf}}}{\mathrm{C}_{\mathrm{oxf}}}+\left(1+\frac{\mathrm{C}_{\mathrm{Si}}}{\mathrm{C}_{\mathrm{oxf}}}\right) \cdot \Phi_{\mathrm{SF}}-\frac{\mathrm{C}_{\mathrm{Si}}}{\mathrm{C}_{\mathrm{oxf}}} \cdot \Phi_{\mathrm{SB}}-\frac{1 / 2 \cdot \mathrm{Q}_{\mathrm{depl}}+\mathrm{Q}_{\mathrm{invf}}}{\mathrm{C}_{\mathrm{oxf}}} \\
& \mathrm{V}_{\mathrm{GB}}=\Phi_{\mathrm{MSB}}-\frac{\mathrm{Q}_{\mathrm{oxb}}}{\mathrm{C}_{\mathrm{oxb}}}+\left(1+\frac{\mathrm{C}_{\mathrm{Si}}}{\mathrm{C}_{\mathrm{oxb}}}\right) \cdot \Phi_{\mathrm{SB}}-\frac{\mathrm{C}_{\mathrm{Si}}}{\mathrm{C}_{\mathrm{oxb}}} \cdot \Phi_{\mathrm{SF}}-\frac{1 / 2 \cdot \mathrm{Q}_{\mathrm{depl}}+\mathrm{Q}_{\mathrm{SB}}}{\mathrm{C}_{\mathrm{oxb}}}
\end{aligned}
$$

onde $Q_{\text {oxb }}$ é a densidade de cargas fixas no óxido enterrado, $Q_{\text {depl }}$ é a quantidade de carga de depleção na camada de silício e Q $\mathrm{SB}_{\mathrm{SB}}$ a carga de inversão.

A tensão de limiar do dispositivo SOI varia dependendo da condição de operação da segunda interface, podendo ter as seguintes equações (COLINGE, 2004):

a) Segunda interface em acumulação $\left(\mathrm{V}_{\text {thf,acc }}\right)$, onde $\Phi_{\mathrm{SB}}=0, \Phi_{\mathrm{SF}}=2 \Phi_{\mathrm{F}}$ e $\mathrm{Q}_{\text {invf }}=0$.

$$
\mathrm{V}_{\mathrm{thf}, \mathrm{acc}}=\Phi_{\mathrm{MSF}}-\frac{\mathrm{Q}_{\mathrm{oxf}}}{\mathrm{C}_{\mathrm{oxf}}}+\left(1+\frac{\mathrm{C}_{\mathrm{Si}}}{\mathrm{C}_{\mathrm{oxf}}}\right) \cdot 2 \Phi_{\mathrm{F}}-\frac{\mathrm{Q}_{\mathrm{depl}}}{2 \mathrm{C}_{\mathrm{oxf}}}
$$


b) Segunda interface em inversão $\left(V_{\text {thf,inv }}\right)$, onde $\Phi_{\mathrm{SB}}=2 \Phi_{\mathrm{F}}, \Phi_{\mathrm{SF}}=2 \Phi_{\mathrm{F}}$ e $\mathrm{Q}_{\text {invf }}=0$.

$$
\mathrm{V}_{\text {thf,inv }}=\Phi_{\mathrm{MSF}}-\frac{\mathrm{Q}_{\mathrm{oxf}}}{\mathrm{C}_{\mathrm{oxf}}}+2 \Phi_{\mathrm{F}}-\frac{\mathrm{Q}_{\mathrm{depl}}}{2 \mathrm{C}_{\mathrm{oxf}}}
$$

c) Segunda interface em depleção $\left(\mathrm{V}_{\text {thf,depl }}\right)$, onde $0<\Phi_{\mathrm{SB}}<2 \Phi_{\mathrm{F}}, \Phi_{\mathrm{SF}}=2 \Phi_{\mathrm{F}}$ e $\mathrm{Q}_{\text {invf }}=0$.

$$
\mathrm{V}_{\text {thf,depl }}=\mathrm{V}_{\text {thf,acc }}-\frac{\mathrm{C}_{\mathrm{Si}} \cdot \mathrm{C}_{\mathrm{oxb}}}{\mathrm{C}_{\mathrm{oxf}}\left(\mathrm{C}_{\mathrm{Si}}+\mathrm{C}_{\mathrm{oxb}}\right)} \cdot\left(\mathrm{V}_{\mathrm{GB}}-\mathrm{V}_{\mathrm{GB}, \mathrm{acc}}\right)
$$

onde $\mathrm{V}_{\mathrm{GB} \text {,acc }}$ é a tensão aplicada no substrato para que a segunda interface acumule.

\subsubsection{INCLINAÇÃO DE SUBLIMIAR}

Inclinação de sublimiar (S) é uma das formas de medir a resposta de um MOSFET, principalmente no que diz respeito à sua velocidade de chaveamento. Ao aplicar uma tensão de porta na região de sublimiar, há uma variação da corrente de dreno. A inclinação de sublimiar é a variação da tensão de porta necessária para aumentar em uma década a corrente de dreno (COLINGE, 2002), como pode ser observado na Figura 4.

Figura 4 - Extração da inclinação de sublimiar da curva IDS em função de $V_{G S}$.

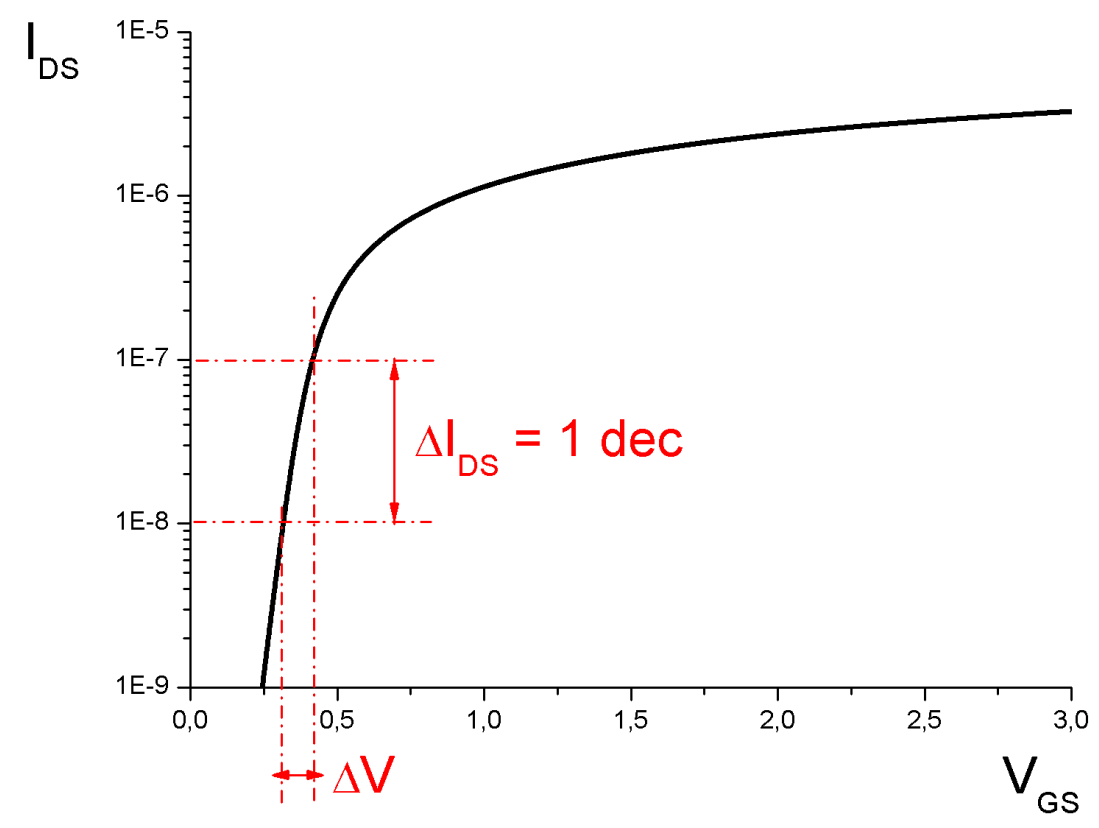

Fonte: Adaptado de Colinge, 2002.

Assim, a inclinação de sublimiar pode ser expressa por:

$$
\mathrm{S}=\frac{\partial \mathrm{V}_{\mathrm{GF}}}{\partial\left(\log \mathrm{I}_{\mathrm{DS}}\right)}
$$


Utilizando a equação da corrente de dreno em sublimiar resulta em (BREWS, 1979):

$$
\mathrm{S}=\mathrm{n} \cdot \frac{\mathrm{k} \cdot \mathrm{T}}{\mathrm{q}} \cdot \ln (10)
$$

Pode-se notar que a inclinação de sublimiar é diretamente proporcional à temperatura e ao fator de corpo. Como o transistor SOI totalmente depletado apresenta menor fator de corpo em comparação com dispositivos convencionais (apresentado na seção 2.3), tem-se uma menor inclinação de sublimiar para estes, possibilitando a criação de dispositivos com menores valores de tensão de limiar (COLINGE, 2004).

Como exemplo tem-se a comparação entre um SOI nMOSFET totalmente depletado com $t_{\mathrm{Si}}=100 \mathrm{~nm}$ e um parcialmente depletado com $\mathrm{t}_{\mathrm{Si}}=200 \mathrm{~nm}$, os quais apresentam uma inclinação de sublimiar de $63 \mathrm{mV} /$ dec e $102 \mathrm{mV} / \mathrm{dec}$ respectivamente, conforme pode ser observado na Figura 5 (COLINGE, 2004).

Figura 5 - Inclinação de sublimiar de um transistor SOI nMOSFET totalmente depletado com $\mathrm{t}_{\mathrm{Si}}=100 \mathrm{~nm}$ e um parcialmente depletado com $\mathrm{t}_{\mathrm{Si}}=200 \mathrm{~nm}$.

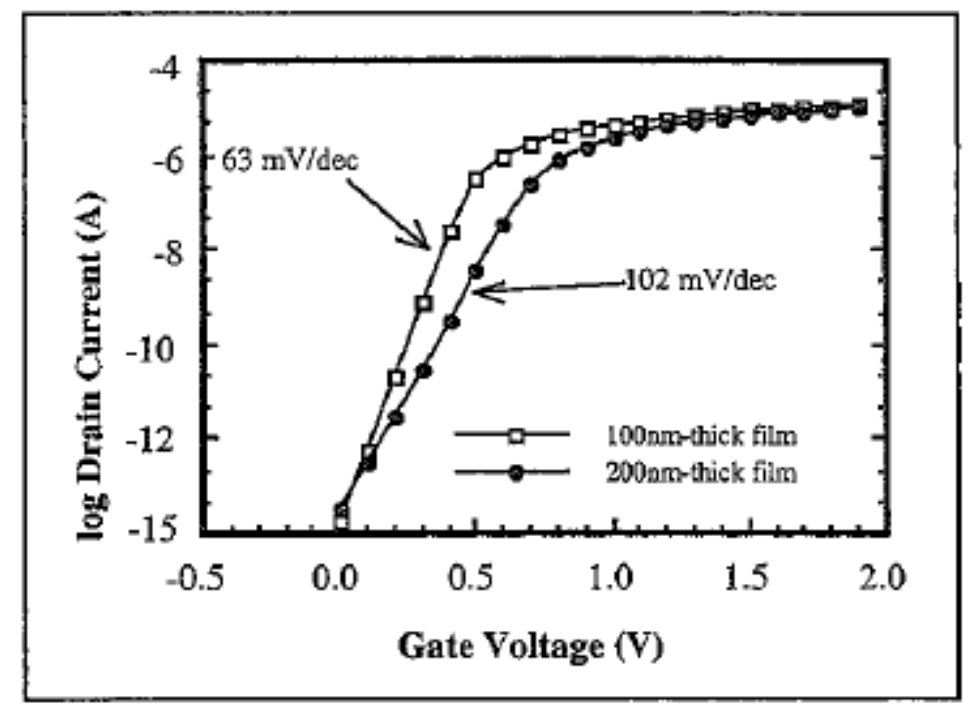

Fonte: Colinge, 2004.

\subsubsection{EFEITO DE CANAL CURTO}

MOSFETs com comprimento de canal reduzido sofrem grande influência das regiões de depleção da fonte e do dreno, que se tornam significativas em relação à região de depleção induzida pela porta. Em um transistor de canal longo, as cargas de depleção de fonte e dreno 
são desprezíveis face ao comprimento de canal, e a carga de depleção controlada pela porta é aproximadamente retangular, dada por $\mathrm{Q}_{\mathrm{depl}}=-\mathrm{q} \cdot \mathrm{N}_{\mathrm{A}} \cdot \mathrm{x}_{\mathrm{dmax}} \quad(\mathrm{SZE}, 1981$; VEERARAGHAVAN, 1988). Para dispositivos de canal curto, as depleções de fonte e dreno tornam-se significativas, e a carga de depleção controlada pela porta será uma fração de $Q_{\text {depl }}$ $\left(Q_{\text {depl,ef }}\right)$, como pode ser observado na Figura 6.

Figura 6 - Perfil transversal de MOSFETs convencionais.
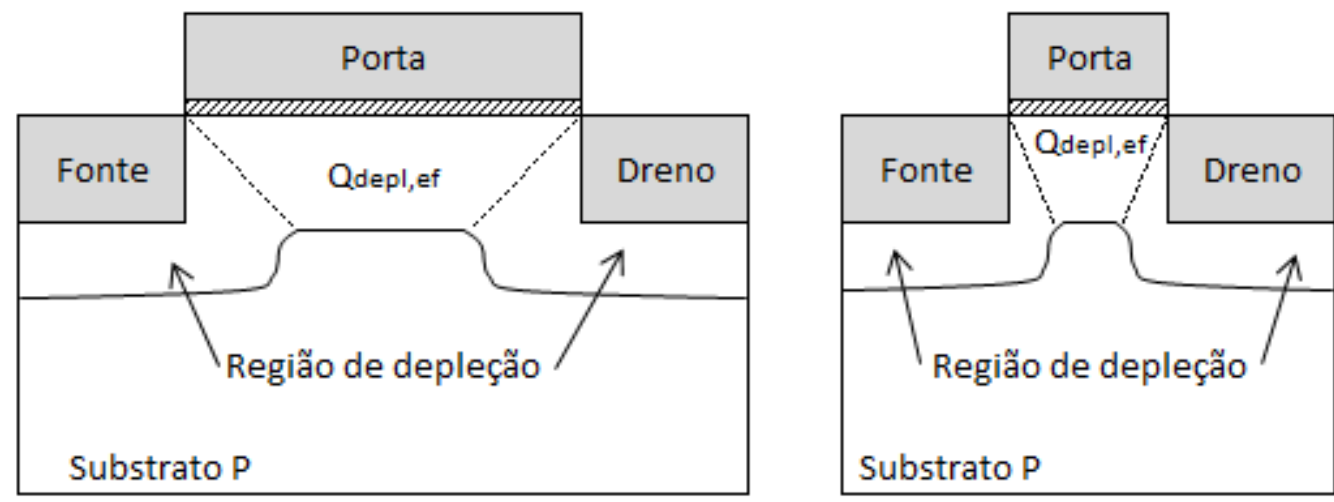

Fonte: Adaptado de Souza, 2005.

Legenda: Transistor de canal longo à esquerda e de canal curto à direita.

A tensão de limiar dos dispositivos é diretamente proporcional à carga de depleção, conforme apresentado na seção 2.4.1. Como a carga de depleção efetiva de dispositivos de canal curto é menor que dispositivos de canal longo, haverá uma redução da tensão de limiar e um aumento da inclinação de sublimiar. Estes são alguns dos efeitos chamados de efeitos de canal curto (VEERARAGHAVAN, 1988; SZE, 1981; MULLER, 1986).

Essa redução da tensão de limiar pode ser observada na Figura 7. 
Figura 7 - Tensão de limiar em função do comprimento de canal de um dispositivo Bulk e um SOI nMOSFET totalmente depletado.

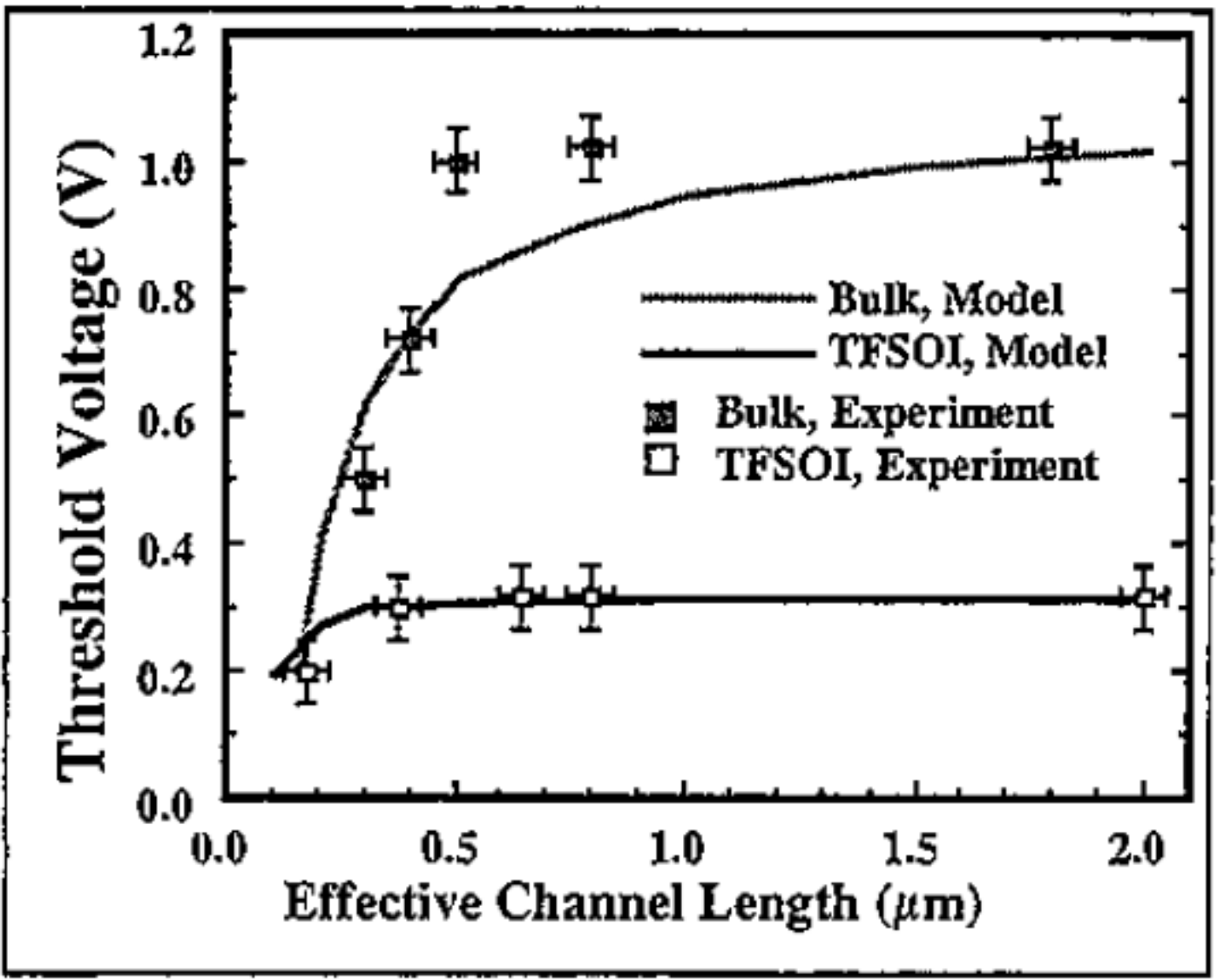

Fonte: Colinge, 2004.

Dispositivos SOI MOSFETs totalmente depletados sofrem menor influência do efeito de canal curto, já que a porcentagem de carga total que é controlada efetivamente pela porta é maior que MOSFETs convencionais como mostra a Figura 8 (SOUZA, 2008).

Figura 8 - Perfil transversal de SOI MOSFETs totalmente depletados.
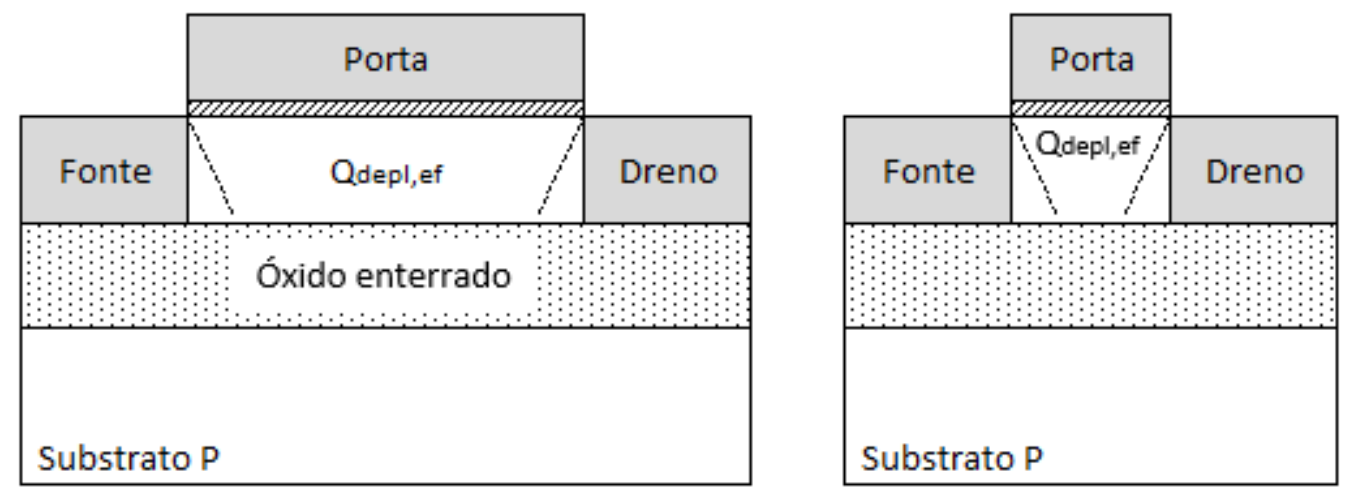

Fonte: Adaptado de Souza, 2005.

Legenda: Transistor de canal longo à esquerda e de canal curto à direita. 


\subsubsection{MOBILIDADE}

A mobilidade de portadores é um parâmetro relacionado ao movimento dos mesmos no semicondutor. Ela influencia diretamente no nível de corrente de dreno que o transistor consegue fornecer. A mobilidade dos portadores diminui conforme o a concentração de dopantes aumenta, como resultado de diversos mecanismos de espalhamento que limitam o movimento dos portadores no interior do semicondutor (SOUZA, 2008).

A tensão aplicada no terminal de porta no SOI MOSFET gera um campo elétrico vertical perpendicular ao canal, que atrai os portadores para a interface silício-óxido de porta. Devido à rugosidade superficial, ocorre a redução da mobilidade. Quanto maior o campo elétrico vertical, maior será a degradação da mobilidade (YOSHIMI, 1989).

Os SOI MOSFETs totalmente depletados apresentam um campo elétrico vertical menor em relação ao MOSFET convencional, o que reduz a degradação da mobilidade e consequentemente apresenta maior mobilidade efetiva ( GUTIERREZ, 1991).

A mobilidade efetiva, degradada pelo campo elétrico vertical, pode ser expressa por (VEERARAGHAVAN, 1988):

$$
\mu_{\mathrm{n}, \mathrm{eff}}=\frac{\mu_{0}}{1+\alpha_{\mathrm{s}} . \mathrm{E}_{\mathrm{eff}} \mid}
$$

onde $\alpha_{\mathrm{s}}$ é o constante de espalhamento, $\mu_{0}$ é a mobilidade dos portadores independente de campo elétrico e $E_{\text {eff }} \mathrm{o}$ campo elétrico efetivo nas direções vertical e horizontal, descrito por $\mathrm{E}_{\mathrm{eff}}=\frac{2 \cdot \Phi_{\mathrm{F}}+\left(\mathrm{V}_{\mathrm{S}}+\mathrm{V}_{\mathrm{DE}}\right) / 2}{\mathrm{t}_{\mathrm{Si}}}+\mathrm{B}-\frac{\left(\mathrm{Q}_{\mathrm{S}}+\mathrm{Q}_{\mathrm{D}}\right) / 2}{2 \cdot \varepsilon_{\mathrm{Si}}}$ onde $\mathrm{B}=-\frac{\mathrm{Q}_{\mathrm{depl}}}{2 \cdot \mathrm{C}_{\mathrm{oxb}} \cdot \mathrm{t}_{\mathrm{Si}}}\left(\frac{1}{1+\mathrm{C}_{\mathrm{Si}} \cdot \mathrm{C}_{\mathrm{oxb}}}+1\right)-\frac{\mathrm{V}_{\mathrm{GB}}}{\left(1+\frac{\mathrm{C}_{\mathrm{Si}}}{\mathrm{C}_{\mathrm{oxb}}}\right) \cdot \mathrm{t}_{\mathrm{Si}}}$, V $\mathrm{DE}$ é o potencial efetivo no canal próximo ao dreno, Qs e QD são as densidades de carga de inversão por unidade de área na fronteira do canal com as regiões de fonte e dreno, respectivamente.

\subsection{CARACTERÍSTICAS ANALÓGICAS}

Considerando um amplificador de tensão, conforme apresentado na Figura 9, em configuração de fonte comum e com carga capacitiva $\left(\mathrm{C}_{\mathrm{L}}\right)$, polarizado por uma fonte de 
corrente constante $\left(\mathrm{I}_{\text {bias }}\right)$, a parcela alternada $\left(\mathrm{V}_{\mathrm{gf}}\right)$, da tensão aplicada à porta $\left(\mathrm{V}_{\mathrm{IN}}\right)$, é amplificada na tensão de saída (V VUT), compondo a parcela alternada ( $\left.\mathrm{V}_{\mathrm{ds}}\right)$ (LAKER, 1994).

Figura 9 - Amplificador de tensão com um único transistor MOS.

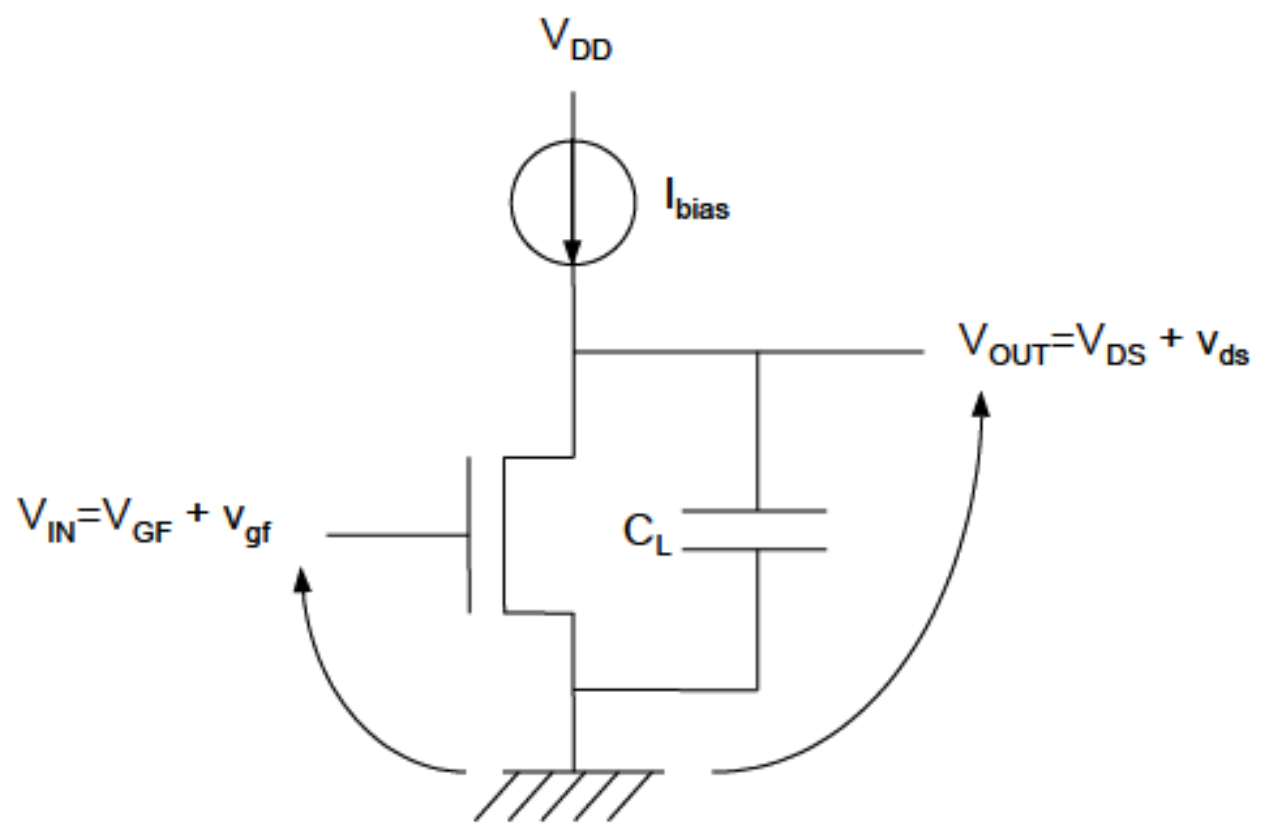

Fonte: Souza, 2008.

O ganho de tensão de malha aberta de baixa frequência $\left(A_{V}\right)$ é dado por (SILVEIRA, 1996):

$$
A_{V}=\frac{v_{d s}}{v_{g f}}=\frac{g_{m}}{g_{D}}
$$

onde $g_{D}$ é a condutância de dreno e $g_{m}$ é a transcondutância.

Na região de saturação, o ganho pode ser aproximado por (SILVEIRA, 1996):

$$
A_{V}=\frac{g_{m}}{I_{D S}} \cdot\left|V_{E A}\right|
$$

onde $\mathrm{V}_{\mathrm{EA}}$ é a tensão de Early. 


\subsubsection{TRANSCONDUTÂNCIA E RAZÃO $\mathrm{g}_{\mathrm{m}} / \mathrm{I}_{\mathrm{DS}}$}

Transcondutância é a medida da eficácia do controle da tensão de porta sobre a corrente de dreno, dado por (COLINGE, 2004):

$$
\mathrm{g}_{\mathrm{m}}=\frac{\partial \mathrm{I}_{\mathrm{DS}}}{\partial \mathrm{V}_{\mathrm{GF}}}
$$

Considerando o modelo de primeira ordem e o modo de operação, a corrente de dreno é dada pelas equações 2 e 3 (LIM, 1984). Utilizando essas equações tem-se as seguintes expressões para a transcondutância (LIM, 1984):

a) triodo:

$$
\mathrm{g}_{\mathrm{m}}=\mu_{\mathrm{n}} \cdot \mathrm{C}_{\mathrm{oxf}} \cdot \frac{\mathrm{w}}{\mathrm{L}} \cdot \mathrm{V}_{\mathrm{DS}}
$$

b) saturação:

$$
\mathrm{g}_{\mathrm{m}}=\frac{\mu_{\mathrm{n}} \cdot \mathrm{C}_{\mathrm{oxf}}}{\mathrm{n}} \cdot \frac{\mathrm{w}}{\mathrm{L}}\left(\mathrm{V}_{\mathrm{GF}}-\mathrm{V}_{\mathrm{thf}}\right)
$$

Como SOI MOSFETs totalmente depletados possuem menor fator de corpo, estes dispositivos apresentam maior transcondutância que os MOSFETs convencionais e SOI MOSFETs parcialmente depletados. Adicionalmente, a transcondutância é diretamente proporcional à mobilidade dos portadores, que é maior nos SOI MOSFETs totalmente depletados (GUTIERREZ, 1991).

A razão $g_{m} / I_{D S}$ é a eficácia de um transistor em converter uma corrente de polarização em transcondutância (VITTOZ, 1994), tendo seu valor máximo em inversão fraca, como pode ser observado na Figura 10: 
Figura 10 - Representação esquemática da razão $\mathrm{g}_{\mathrm{m}} / \mathrm{I}_{\mathrm{DS}}$ de MOSFETs.

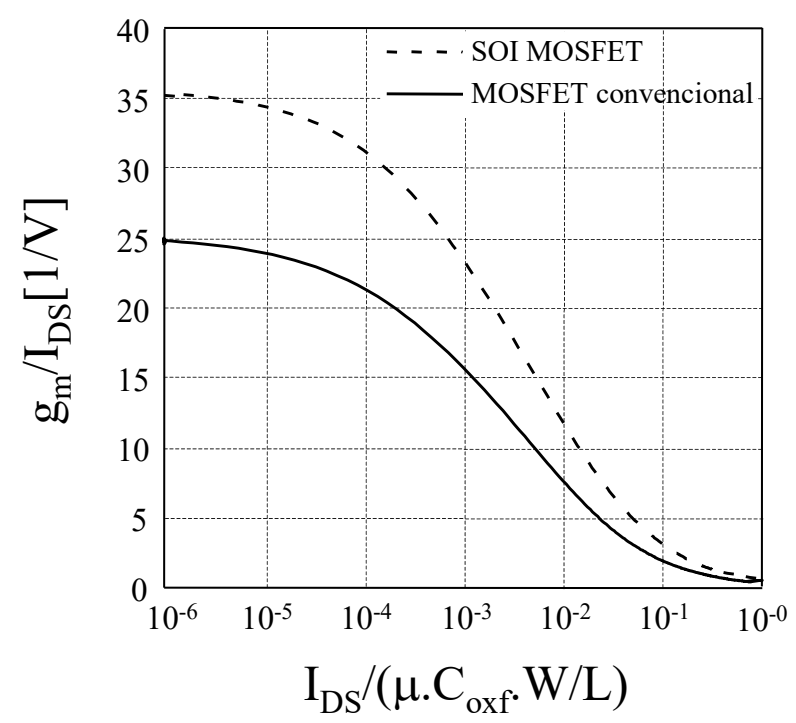

Fonte: Souza, 2005.

Legenda: Transistor nMOSFET convencional linha cheia e SOI MOSFET de camada fina linha tracejada.

A relação $g_{m} / I_{D S}$ em inversão fraca pode ser expressa pela equação (VITTOZ, 1994). Através desta equação, pode-se notar que o valor máximo de $\mathrm{g}_{\mathrm{m}} / \mathrm{I}_{\mathrm{DS}}$ está relacionado ao inverso da inclinação de sublimiar.

$$
\frac{g_{m}}{I_{D S}}=\frac{q}{\text { n.k.T }}
$$

Em inversão forte, $\mathrm{g}_{\mathrm{m}} / \mathrm{I}_{\mathrm{DS}}$ pode ser expressa por (FLANDRE, 1996):

$$
\frac{\mathrm{g}_{\mathrm{m}}}{\mathrm{I}_{\mathrm{DS}}}=\sqrt{\frac{2 \cdot \mu_{\mathrm{n}} \cdot \mathrm{C}_{\mathrm{oxf}} \cdot \frac{\mathrm{w}}{\mathrm{L}}}{\mathrm{n} \cdot \mathrm{I}_{\mathrm{DS}}}}
$$

Devido à presença do fator de corpo nas equações, e conforme discutido anteriormente, sabe-se que o efeito de corpo (n) de SOI MOSFETs totalmente depletados é menor do que o efeito de corpo de MOSFETs convencionais. Portanto a razão $\mathrm{g}_{\mathrm{m}} / \mathrm{I}_{\mathrm{DS}}$ em SOI MOSFETs totalmente depletados é maior que em SOI MOSFETs parcialmente depletados e transistores MOS convencionais (FLANDRE, 1994). 
Pode-se notar que para o transistor operando em inversão fraca há a dependência inversa em relação à temperatura, o transistor operando em inversão forte há a dependência indireta com a temperatura, através da raiz quadrada da mobilidade. Portanto pode-se observar que a variação da relação $\mathrm{g}_{\mathrm{m}} / \mathrm{I}_{\mathrm{DS}}$ em inversão fraca é maior devido a dependência direta com a temperatura (SOUZA, 2008).

\subsubsection{CONDUTÂNCIA DE DRENO E TENSÃO EARLY}

A condutância de dreno é a variação da corrente de dreno em relação à tensão de dreno, dada por:

$$
\mathrm{g}_{\mathrm{D}}=\frac{\partial \mathrm{I}_{\mathrm{DS}}}{\partial \mathrm{V}_{\mathrm{DS}}}
$$

A condutância de dreno é um parâmetro importante do ponto de vista analógico. Por exemplo, para o cálculo do ganho da tensão, quanto menor a condutância maior será o ganho de saída (PAVANELLO et al., 2000a). Uma vez que a condutância de SOI MOSFETs é menor que a de MOSFETs convencionais, o ganho do primeiro será maior (PAVANELLO, 2000).

Uma outra maneira de avaliar a condutância de dreno é através da tensão Early. A tensão Early é o parâmetro usado para quantificar a inclinação da curva IDS x VDS na região de saturação. Em uma primeira aproximação, pode ser obtida através da extrapolação de uma reta tangente à curva na saturação, até a intersecção do eixo $\mathrm{V}_{\mathrm{DS}}$, resultando em uma corrente nula, conforme ilustrado na Figura 11 (SOUZA, 2005). 
Figura 11 - Determinação da tensão Early através da extrapolação das curvas de corrente de dreno na saturação em função da tensão de dreno.

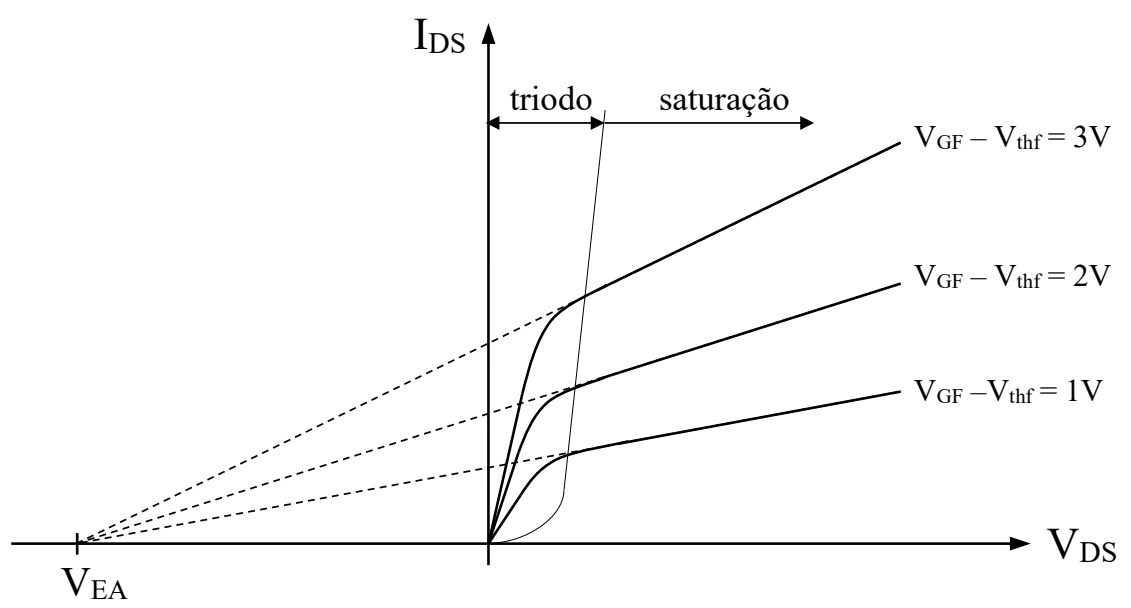

Fonte: Souza, 2005.

Em um MOSFET, quando a tensão de dreno é maior que a tensão de saturação, a região de depleção do dreno aumenta, diminuindo o comprimento efetivo do canal do transistor, como pode ser observado na Figura 12. Esta redução do comprimento efetivo provoca o aumento da corrente de dreno de saturação (IDSsat). Este acréscimo de corrente faz com que a corrente de saturação, idedalmente constante, apresente um aumento, fazendo com que a inclinação da curva $I_{D S} x V_{D S}$ aumente na região de saturação (COLINGE, 2002). A este efeito de redução do comprimento de canal causada pelo aumento da região de depleção induzida pela tensão aplicada ao dreno, dá-se o nome de efeito de modulação de comprimento de canal. 
Figura 12 - Perfil transversal de um SOI nMOSFET sofrendo modulação do comprimento de canal.

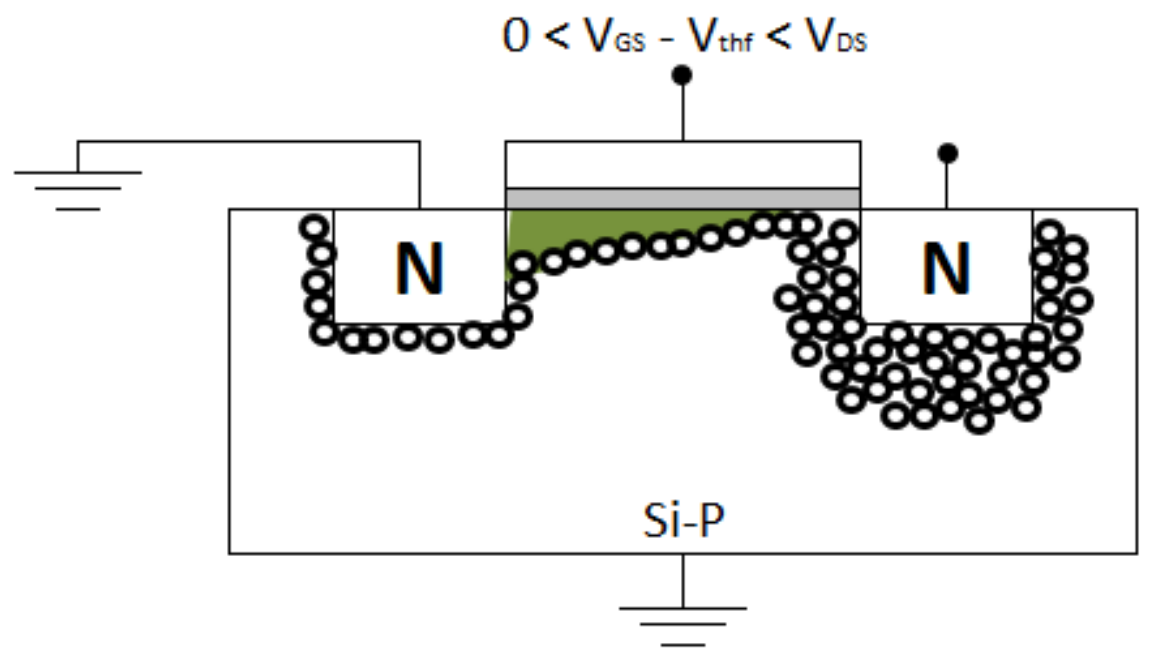

Fonte: Adaptado de Martino, 2004.

A equação de corrente de dreno simplificada, incluindo o efeito de modulação do comprimento de canal pela tensão de dreno, é dada por (SEDRA, 2007):

$$
\mathrm{I}_{\mathrm{DSsat}}=\frac{\mathrm{W} \cdot \mu_{\mathrm{n}} \cdot \mathrm{C}_{\mathrm{oxf}}}{2 \cdot \mathrm{L}} \cdot\left(\mathrm{V}_{\mathrm{GF}}-\mathrm{V}_{\mathrm{thf}}\right)^{2} \cdot\left(1+\lambda \mathrm{V}_{\mathrm{DS}}\right)
$$

onde $\lambda$ é o parâmetro dependente do processo de fabricação e relacionada com a modulação do comprimento de canal com a tensão de dreno.

Como a tensão Early varia com $\mathrm{V}_{\mathrm{DS}}$, sua extração é feita na região de saturação (COLINGE, 2002):

$$
\left|V_{\text {EA }}\right| \cong \frac{I_{\text {DSsat }}}{g_{\text {Dsat }}}
$$

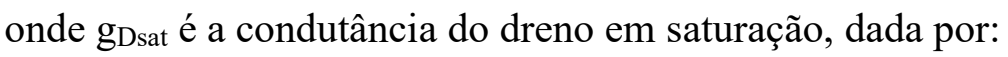

$$
\mathrm{g}_{\text {Dsat }}=\frac{\mathrm{dI}_{\mathrm{DSsat}}}{\mathrm{dV}_{\mathrm{DS}}} \cong \frac{\mathrm{W} \cdot \mu_{\mathrm{n}} \cdot \mathrm{C}_{\mathrm{oxf}}}{2 \cdot \mathrm{L}} \cdot\left(\mathrm{V}_{\mathrm{GF}}-\mathrm{V}_{\mathrm{thf}}\right)^{2} \cdot \lambda
$$

A tensão Early em transistores SOI e MOS convencionais é basicamente a mesma, porém tem-se um maior ganho de tensão em transistores SOI totalmente depletado devido à maior relação $\mathrm{g}_{\mathrm{m}} / \mathrm{I}_{\mathrm{DS}}$ como mostrado anteriormente (COLINGE, 2002). 


\subsubsection{DESCASAMENTO ENTRE MOSFETS}

O descasamento entre MOSFETs é a diferença de desempenho entre dispositivos que foram feitos através do mesmo projeto de leiaute e com mesmas dimensões. A fabricação de dispositivos idênticos (casados) é extremamente importante para o desempenho elétrico de circuitos integrados analógicos, tais como pares diferenciais e espelhos de corrente (LAKER, 1994).

O descasamento relativo da corrente de dreno $\left(\Delta \mathrm{I}_{\mathrm{DS}} / \mathrm{I}_{\mathrm{DS}}\right)$ é expresso em função do descasamento da tensão de limiar $\left(\Delta \mathrm{V}_{\text {thf }} / \mathrm{V}_{\text {thf }}\right)$ e do fator de corrente $(\Delta \beta / \beta)$, dado por (CROON, 2004):

$$
\frac{\Delta \mathrm{I}_{\mathrm{DS}}}{\mathrm{I}_{\mathrm{DS}}}=\frac{-\Delta \mathrm{V}_{\mathrm{thf}}}{\mathrm{V}_{\mathrm{GF}}-\mathrm{V}_{\mathrm{thf}}}+\frac{\Delta \beta}{\beta}
$$

A variação da tensão de limiar de um SOI MOSFET se dá pela variação da concentração de dopantes do canal, espessura da camada de silício e do óxido de porta. Essa variação é a principal componente no descasamento da corrente de dreno em inversão fraca (CROON, 2002).

Em tecnologias modernas, as variações da espessura do óxido de porta e da camada de silício são muito pequenas, portanto a principal responsável pela variação da tensão de limiar é a variação da concentração de dopantes (LAKER, 1994).

Estudos apontam que a variação da tensão de limiar juntamente com a variação do fator de corpo influenciam na variação da corrente de dreno. O fator de corpo para SOI MOSFETs totalmente depletados permanece constante, somente em inversão muito fraca ou quando a temperatura aumenta que o fator $\mathrm{n}$ começa a aumentar. Se o potencial de superfície ficar abaixo de 2. $\Phi_{\mathrm{F}}$, o fator de corpo irá aumentar e apresentará forte dependência com a tensão de porta, conforme mostrado na equação 31 (VANCAILLIE, 2003).

$$
\frac{\Delta \mathrm{I}_{\mathrm{DS}}}{\mathrm{I}_{\mathrm{DS}}}=-\frac{\mathrm{g}_{\mathrm{m}}}{\mathrm{I}_{\mathrm{DS}}} \cdot \Delta \mathrm{V}_{\mathrm{th}}+\mathrm{f}\left(\mathrm{V}_{\mathrm{GF}}\right) \cdot \Delta \mathrm{n}
$$

sendo $f\left(V_{G F}\right)=\frac{1}{\left[\alpha_{1}+\left(\frac{v_{G F}}{\alpha_{2}}\right)^{\alpha_{3}}\right]}$, onde $\alpha_{1}, \alpha_{2}$ e $\alpha_{3}$ são parâmetros de ajuste. 
A variação da concentração efetiva de dopantes (CED) é dada por:

$$
\mathrm{N}_{\mathrm{A}, \mathrm{eff}}=\mathrm{N}_{\mathrm{A}}-\frac{2 \cdot \varepsilon_{\mathrm{Si}}}{\mathrm{q}} \cdot \frac{2 \cdot \Phi_{\mathrm{F}}}{\mathrm{L}^{2}}
$$

Pode-se observar que quanto menor o comprimento do canal, maior será o valor da concentração. O descasamento da tensão de limiar depende da variação de dopantes da camada de depleção, e é dado por:

$$
\sigma \mathrm{V}_{\text {thf,CED }}=\sigma \mathrm{V}_{\text {thf }} \cdot \frac{1}{\sqrt{1-\frac{4 . \varnothing \Phi_{\mathrm{F}} \cdot \varepsilon_{\mathrm{Si}}}{\mathrm{q} \cdot \mathrm{N}_{\mathrm{A}} \cdot \mathrm{L}^{2}}}}
$$

onde $\sigma \mathrm{V}_{\text {thf,CED }}$ é o desvio padrão da tensão de limiar em relação à concentração efetiva de dopantes e $\sigma \mathrm{V}_{\text {thf }}$ é o desvio padrão da tensão de limiar.

O desvio padrão relativo da tensão de limiar pode ser expresso por (DIFRENZA, 2003):

$$
\frac{\sigma \mathrm{V}_{\text {thf }}}{\mathrm{V}_{\text {thf,mean }}}=\frac{\sigma_{0} \mathrm{~V}_{\text {thf }}}{\mathrm{V}_{\text {thf,mean }} \sqrt{\mathrm{WxL}}}
$$

onde $\sigma_{0} \mathrm{~V}_{\text {thf }}$ é o desvio padrão da tensão de limiar normalizado em relação à área de porta $\mathrm{e}$ $\mathrm{V}_{\text {thf,mean }}$ é a tensão de limiar média.

Pode-se observar que quanto maior a área do transistor menor será a variação da tensão de limiar. Como a tensão de limiar está relacionada à concentração de dopantes, espessura da camada de silício e espessura do óxido de porta, tem-se a equação (PELGROM, 1998):

$$
\sigma_{0} \mathrm{~V}_{\mathrm{thf}}=\frac{\mathrm{q} \cdot \mathrm{t}_{\mathrm{oxf}} \cdot \sqrt{2 \cdot \mathrm{N}_{\mathrm{A}} \cdot \mathrm{t}_{\mathrm{Si}}}}{\varepsilon_{0} \cdot \varepsilon_{\mathrm{ox}}}
$$

A variação do fator de corrente $(\Delta \beta / \beta)$ é um importante fator no descasamento da corrente de dreno, já que o mesmo sofre influência da variação da concentração de dopantes e dos valores do comprimento e largura do transistor (CROON, 2004; DIFRENZA, 2003), conforme pode-se observar pela equação: 


$$
\beta=\mu_{0} \cdot C_{o x} \cdot \frac{W}{L}
$$

A variação da concentração de dopantes irá causar variação da mobilidade dos portadores, fazendo com que o fator de corrente sofra uma variação. Assim como as variações de comprimento e largura do MOSFET, a variação da concentração de dopantes influenciará o fator de corrente. De forma geral, a variação do comprimento do transistor é mais importante pois suas dimensões são menores se comparadas com a largura (CROON, 2004).

\subsection{TRANSISTOR DE CANAL GRADUAL}

Os SOI MOSFETs totalmente depletados apresentam ótimos resultados como aumento da mobilidade de portadores, menores dimensões de comprimento de canal efetivo, aumento da transcondutância, simplificação dos processos de fabricação, diminuição das capacitâncias parasitárias de fonte e dreno, diminuição dos efeitos de canal curto (FLANDRE, 1994; COLINGE, 1998), entre outras.

Apesar disto existem algumas desvantagens em relação aos dispositivos MOSFETs convencionais, como o alto custo de fabricação da lâmina SOI e a dependência da tensão de limiar com a concentração de dopantes e com a espessura da camada de silício (SOUZA, 2008), entre outras. No que diz respeito ao comportamento analógico, ocorre a diminuição da tensão de ruptura devido à presença do corpo flutuante que ativa o transistor bipolar parasitário.

O efeito bipolar parasitário é influenciado pela quantidade de lacunas geradas por ionização por impacto devido ao alto campo elétrico na junção canal-dreno. A geração dessas lacunas depende da tensão aplicada ao dreno, da espessura da camada de silício e da concentração de dopantes na região de canal (SZE, 1981). Uma forma utilizada para reduzir esse efeito é a diminuição da concentração de dopantes, reduzindo a barreira potencial e, consequentemente, o campo elétrico. A diminuição do campo elétrico vertical diminui o número de portadores gerados na ionização por impacto, e consequentemente a corrente de polarização do transistor bipolar parasitário. Essa técnica é chamada de (LDD) (SANCHEZ, 1989). Entretanto, a redução da concentração de dopantes na região de dreno traz como desvantagem o aumento da resistência série associada ao transistor, que já é elevada em transistores SOI devido à redução da espessura da camada de silício (KISTLER, 1992; JENG, 1990). 
Posteriormente, foi proposta uma nova estrutura SOI totalmente depletado com perfil assimétrico de dopantes na região de canal, chamada de transistor SOI MOSFET de canal gradual ou GC SOI MOSFET (PAVANELLO, 1999).

Nos GC SOI MOSFET a região de corpo é dividida em duas regiões com concentrações de dopantes diferentes, sendo fortemente dopada junto à fonte e fracamente dopada junto ao dreno. A região fortemente dopada é chamada de HD, e o comprimento é dado por LHD e sua concentração é $\mathrm{N}_{\mathrm{AH}}$. Esta região tem como função ajustar a tensão de limiar. A região fracamente dopada é chamada de $\mathrm{LD}$, e funciona como uma extensão da região de dreno, com comprimento $\mathrm{L}_{\mathrm{LD}}$, apresentando dopagem natural $\left(\mathrm{N}_{\mathrm{AL}}\right)$ que diminui o efeito de ionização por impacto, devido à redução do campo elétrico vertical.

A Figura 13 apresenta o perfil de uma estrutura GC SOI MOSFET.

Figura 13 - Perfil de um GC SOI MOSFET totalmente depletado.

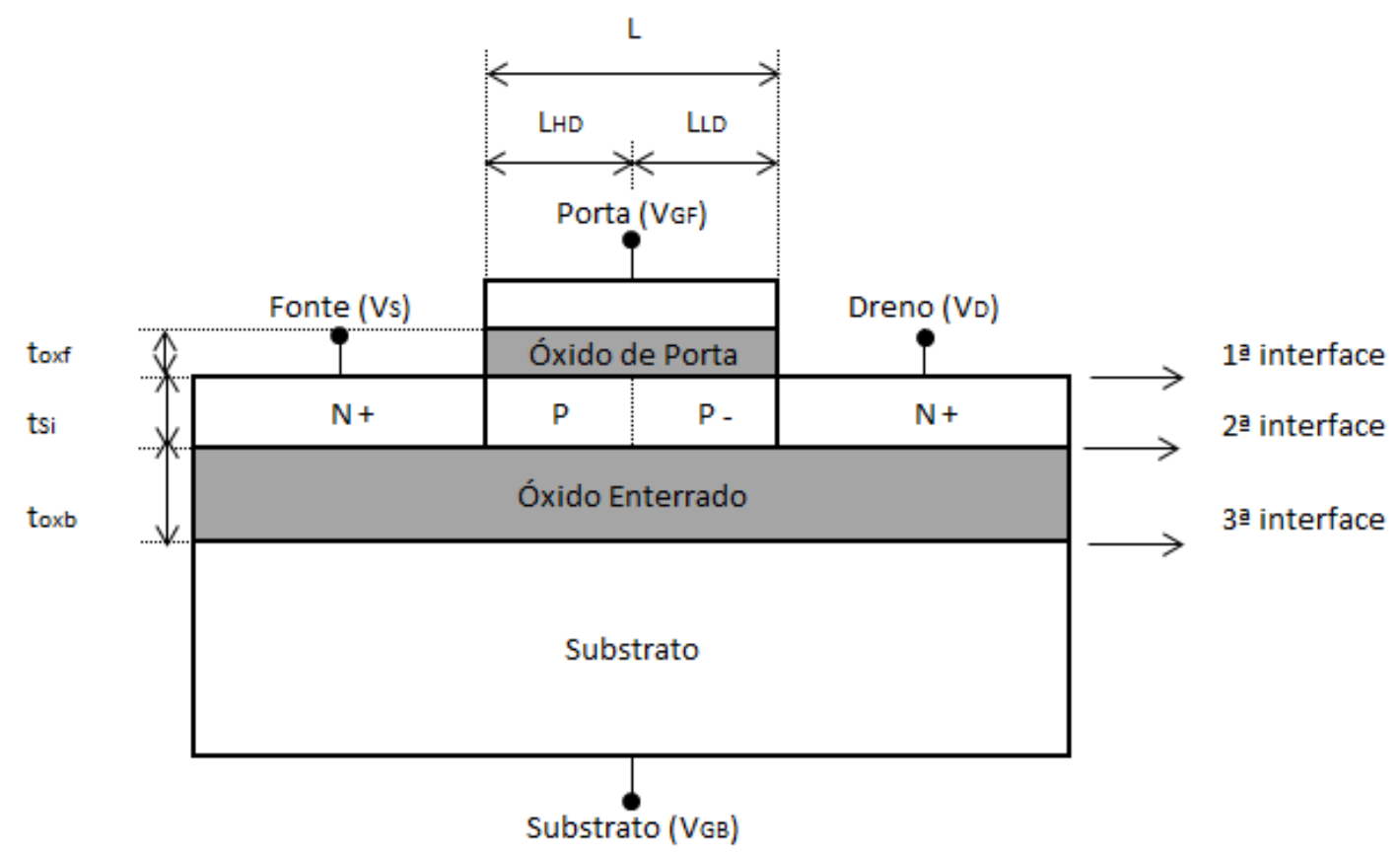

Fonte: Adaptado de Souza, 2008.

Trabalhos comparativos entre dispositivos GC SOI MOSFET e SOI MOSFET uniformemente dopados totalmente depletados demonstram que ao se reduzir o comprimento efetivo do canal há um aumento da corrente de dreno e da transcondutância (PAVANELLO et al, 2000a), conforme pode ser observado na Figura 14 e Figura 15, que apresentam as curvas $\mathrm{g}_{\mathrm{m}}$ em função de $\mathrm{V}_{\mathrm{GF}}$ e $\mathrm{I}_{\mathrm{DS}}$ em função de $\mathrm{V}_{\mathrm{DS}}$. Tal fato deve-se à redução do comprimento efetivo de canal, que ocorre à medida que a relação $\mathrm{L}_{\mathrm{LD}} / \mathrm{L}$ aumenta. $\mathrm{O}$ canal efetivo desse 
transistor é dado por $\mathrm{L}_{\mathrm{eff}}=\mathrm{L}-\mathrm{L}_{\mathrm{LD}}$, onde $\mathrm{L}$ é o comprimento de máscara da porta (SOUZA; FLANDRE, 2008). A região fracamente dopada apresenta tensão de limiar negativa e encontra-se em inversão forte. Para valores de tensão de porta inferiores à tensão de limiar da estrutura, o transistor GC comporta-se de forma semelhante a um transistor SOI convencional de comprimento canal efetivo igual a $\mathrm{L}_{\mathrm{HD}}$ (CERDEIRA, 2005).

Figura 14 - Curvas da transcondutância em função da tensão aplicada à porta.

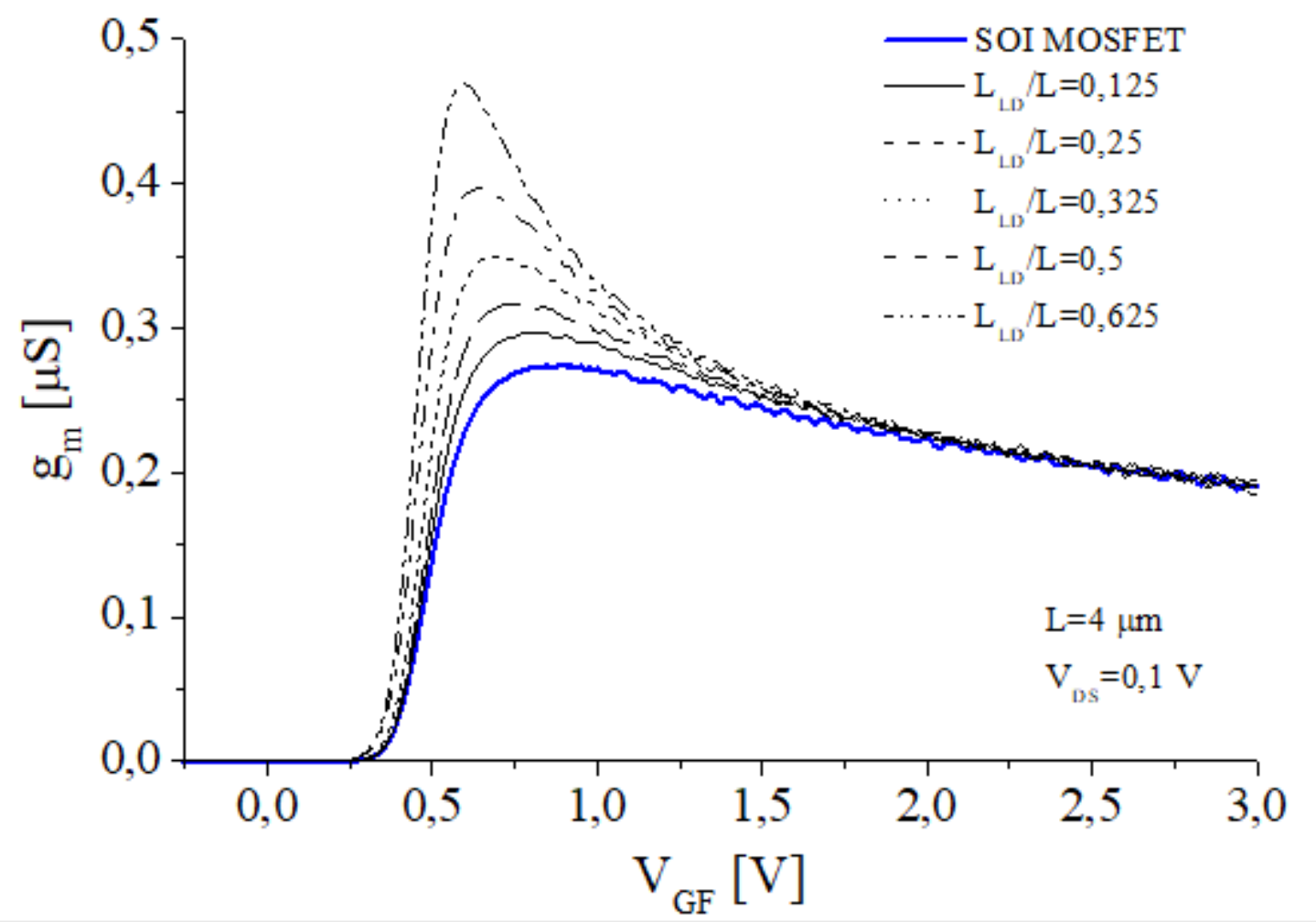

Fonte: Pavanello, 2000.

Legenda: GC SOI nMOSFETs e SOI nMOSFET convencional com L $=4 \mu \mathrm{m}$ e W $=18 \mu \mathrm{m}$. 
Figura 15 - Curvas IDS em função de $V_{D S}$ de dispositivos nMOSFETs.

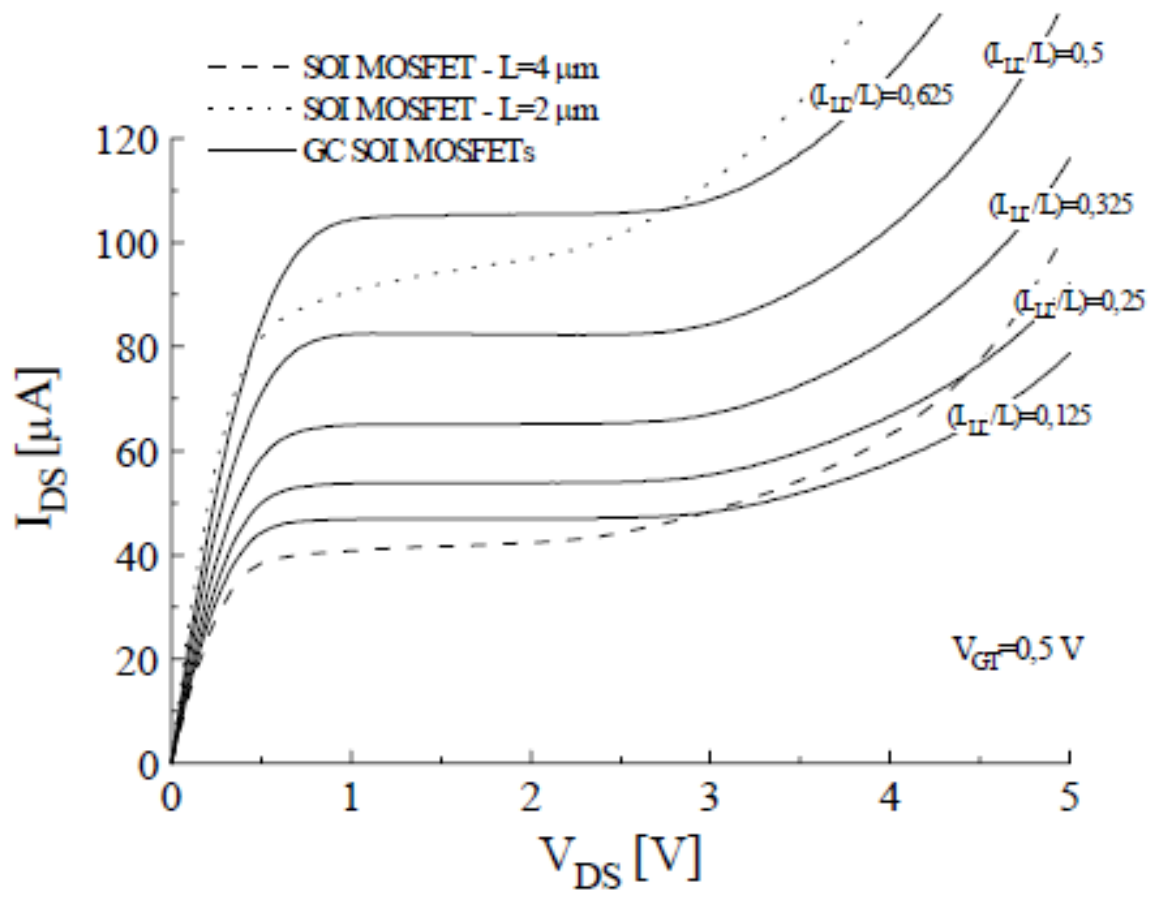

Fonte: Pavanello, 2000.

Legenda: GC SOI nMOSFETs com $\mathrm{L}=4 \mu \mathrm{m}$ e SOI nMOSFETs convencionais com $\mathrm{L}=4 \mu \mathrm{m}$ e $\mathrm{L}=2 \mu \mathrm{m}$, todos com $\mathrm{W}=18 \mu \mathrm{m}$.

Aplicando-se altas tensões de porta e aumentando a relação $\mathrm{L}_{\mathrm{LD}} / \mathrm{L}$ há um aumento na degradação da transcondutância. Isso se dá devido a concentração de elétrons na superfície do canal ao longo de seu comprimento, a qual será influenciada pela tensão aplicada à porta. A região fracamente dopada do canal apresenta tensão de limiar negativa trabalhando em inversão forte (PAVANELLO, 1999).

Para uma tensão de porta menor que a tensão de limiar, os GC SOI MOSFETs, cujo comprimento de canal é representado por $\mathrm{L}_{\mathrm{HD}}$, respondem de forma semelhante a transistores SOI convencionais, com comprimento de canal L, quando $\mathrm{L}=\mathrm{L}_{\mathrm{HD}}$. Conforme aumenta-se a tensão de porta, a concentração de elétrons na região altamente dopada e fracamente dopada ficarão semelhantes, assim o transistor GC SOI se assemelha a um transistor SOI convencional de mesmo comprimento de canal total, porém com maior nível de corrente. Além disso, o aumento da concentração de elétrons degrada a mobilidade da região fracamente dopada, aumentando a degradação da transcondutância (CERDEIRA, 2005).

Adicionalmente, os resultados reportados na literatura mostram a redução da condutância de dreno e, consequentemente, um aumento da tensão Early (PAVANELLO et al, 2000b) nos dispositivos GC SOI, como pode ser observado experimentalmente na Tabela 1. 
Tabela 1 - Tensão Early extraída através de curvas $\mathrm{I}_{\mathrm{DS}}$ em função de $\mathrm{V}_{\mathrm{DS}}$ experimentais.

\begin{tabular}{cc}
\hline $\mathbf{L}_{\mathbf{L D}} / \mathbf{L}$ & $\mathrm{V}_{\mathrm{EA}}[\mathrm{V}]$ \\
\hline $\mathbf{0 , 0 5}$ & $-34,9$ \\
\hline $\mathbf{0 , 1 3}$ & $-81,2$ \\
\hline $\mathbf{0 , 2 8}$ & $-257,8$ \\
\hline $\mathbf{0 , 5 1}$ & $-206,4$ \\
\hline $\mathbf{1 , 0 0}$ & $-11,2$
\end{tabular}

Fonte: Pavanello; Martino, $20 \overline{00 .}$

Legenda: GC SOI nMOSFETs com $\mathrm{L}=2 \mu \mathrm{m}$.

A condutância de dreno é um fator muito importante para o desenvolvimento de projetos de circuitos integrados analógicos. Pode-se notar também, através das curvas da Figura 15, um aumento da tensão de ruptura de dreno (BV $\mathrm{BS}_{\text {) }}$ (PAVANELLO; MARTINO, 2000), assim como pode também ser observado experimentalmente na Tabela 2.

Tabela 2 - Tensão de ruptura extraída de curvas IDS x VDS experimentais.

\begin{tabular}{cc}
\hline $\mathbf{L}_{\mathbf{L D}} / \mathbf{L}$ & $\mathrm{B} V_{\mathrm{DS}}[\mathrm{V}]$ \\
\hline $\mathbf{0 , 1 8}$ & 2,65 \\
\hline $\mathbf{0 , 2 7}$ & 2,58 \\
\hline $\mathbf{0 , 3 9}$ & 2,50 \\
\hline $\mathbf{0 , 4 7}$ & 2,44 \\
\hline $\mathbf{1 , 0 0}$ & 2,13
\end{tabular}

Fonte: Pavanello; Martino, 2000.

Legenda: GC SOI nMOSFETs com $\mathrm{L}=1 \mu \mathrm{m}$.

Poucos trabalhos reportados na literatura apresentam resultados de descasamento em SOI MOSFETs de canal gradual. A corrente de dreno de saída depende diretamente da razão

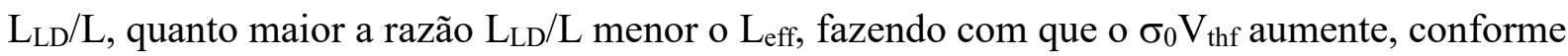
pode-se observar na Figura 16, que apresenta a curva $\sigma_{0} \mathrm{~V}_{\text {thf }} / \mathrm{V}_{\text {thf,mean }}$ em função da razão $1 / \sqrt{\mathrm{W} \mathrm{x} \mathrm{L} \mathrm{Lff}_{\mathrm{eff}}}$. Conforme esperado, quanto maior a área do transistor ( $\left.\mathrm{W} \times \mathrm{L}_{\mathrm{eff}}\right)$, menor a variação da tensão de limiar. 
Figura $16-\sigma_{0} \mathrm{~V}_{\text {thf }} / \mathrm{V}_{\text {thf,mean }}$ em função da razão $1 /\left(\mathrm{W} x \mathrm{~L}_{\mathrm{eff}}\right)^{1 / 2}$ para GC SOI MOSFETs com diversas razões $\mathrm{L}_{\mathrm{LD}} / \mathrm{L}$.

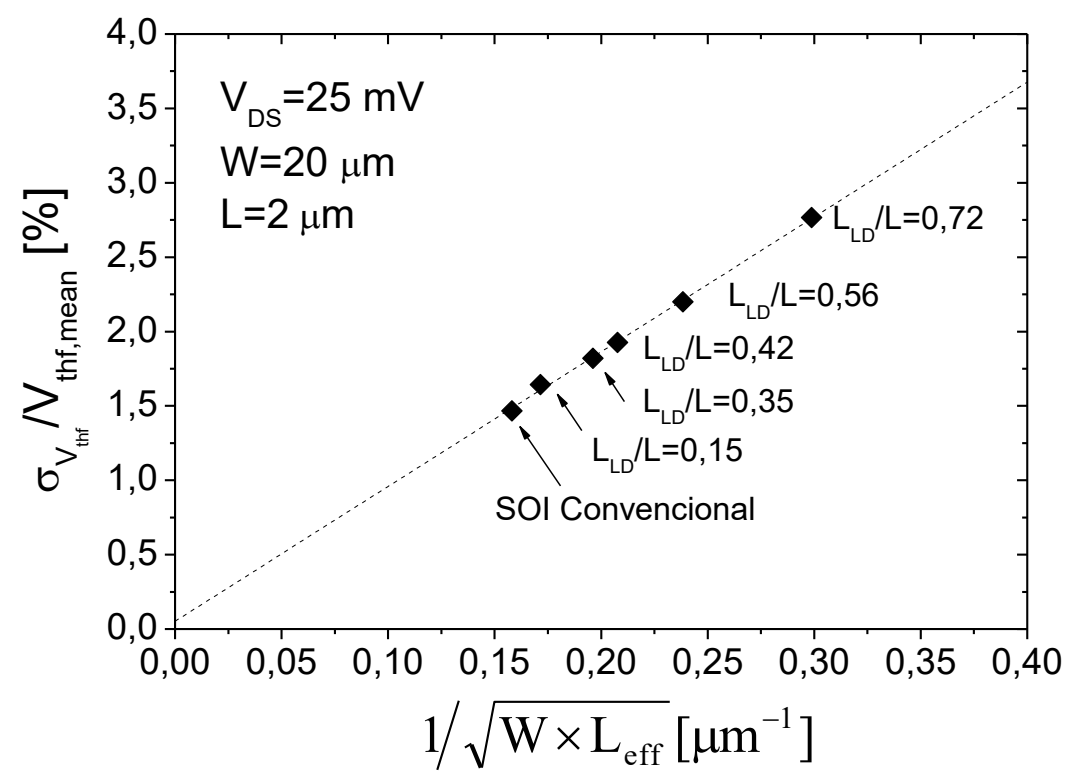

Fonte: Souza, 2008.

Aumentando a razão $\mathrm{LLD}_{\mathrm{LD}} \mathrm{L}$, a variação da corrente de dreno também aumenta, conforme pode ser visto na Figura 17, como resultado da redução do comprimento efetivo de canal.

Figura 17 - Desvio relativo da corrente de dreno em função da corrente normalizada operando em saturação $\left(\mathrm{V}_{\mathrm{DS}}=1,5 \mathrm{~V}\right)$.

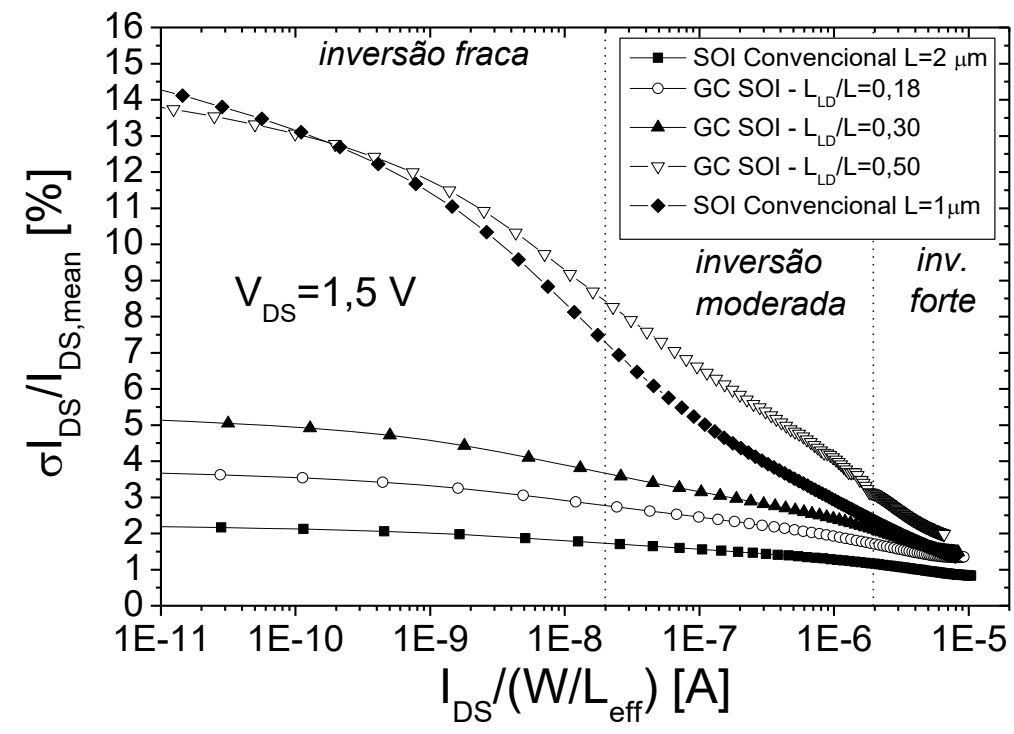

Fonte: Souza, 2008. 


\section{RESULTADOS EXPERIMENTAIS}

Os SOI MOSFETs utilizados nessa etapa foram projetados e fabricados no Laboratório de Microeletrônica da Universidade Católica de Louvain (UCLouvain). Os dispositivos são GC SOI MOSFETs totalmente depletados com comprimento total de canal de $2 \mu \mathrm{m}$ (com relação $\mathrm{L}_{\mathrm{LD}} / \mathrm{L}$ de $0,0.25,0.375$ e 1 , onde 0 e 1 representam os SOI MOSFETs uniformemente dopados com alta e baixa concentração de dopantes, respectivamente), largura de canal de $20 \mu \mathrm{m}$, tsi de $80 \mathrm{~nm}$, toxb de $390 \mathrm{~nm}$, toxf de $31 \mathrm{~nm}$, concentração de dopantes $\mathrm{N}_{\mathrm{AH}}$ de $5,6.10^{16} \mathrm{~cm}^{-3}$ e $\mathrm{N}_{\mathrm{AL}}$ de $1.10^{15} \mathrm{~cm}^{-3}$. Para cada razão $\mathrm{L}_{\mathrm{LD}} / \mathrm{L}$, foi fabricado um grupo de 20 transistores com as mesmas características, com terminais de fonte, porta e dreno independentes, resultando em um total de 80 transistores caracterizados eletricamente. A Figura 18 apresenta o leiaute do chip utilizado. No Apêndice A são apresentados os leiautes dos chips utilizados.

Figura 18 - Foto parcial do chip contendo os SOI MOSFETs utilizados.

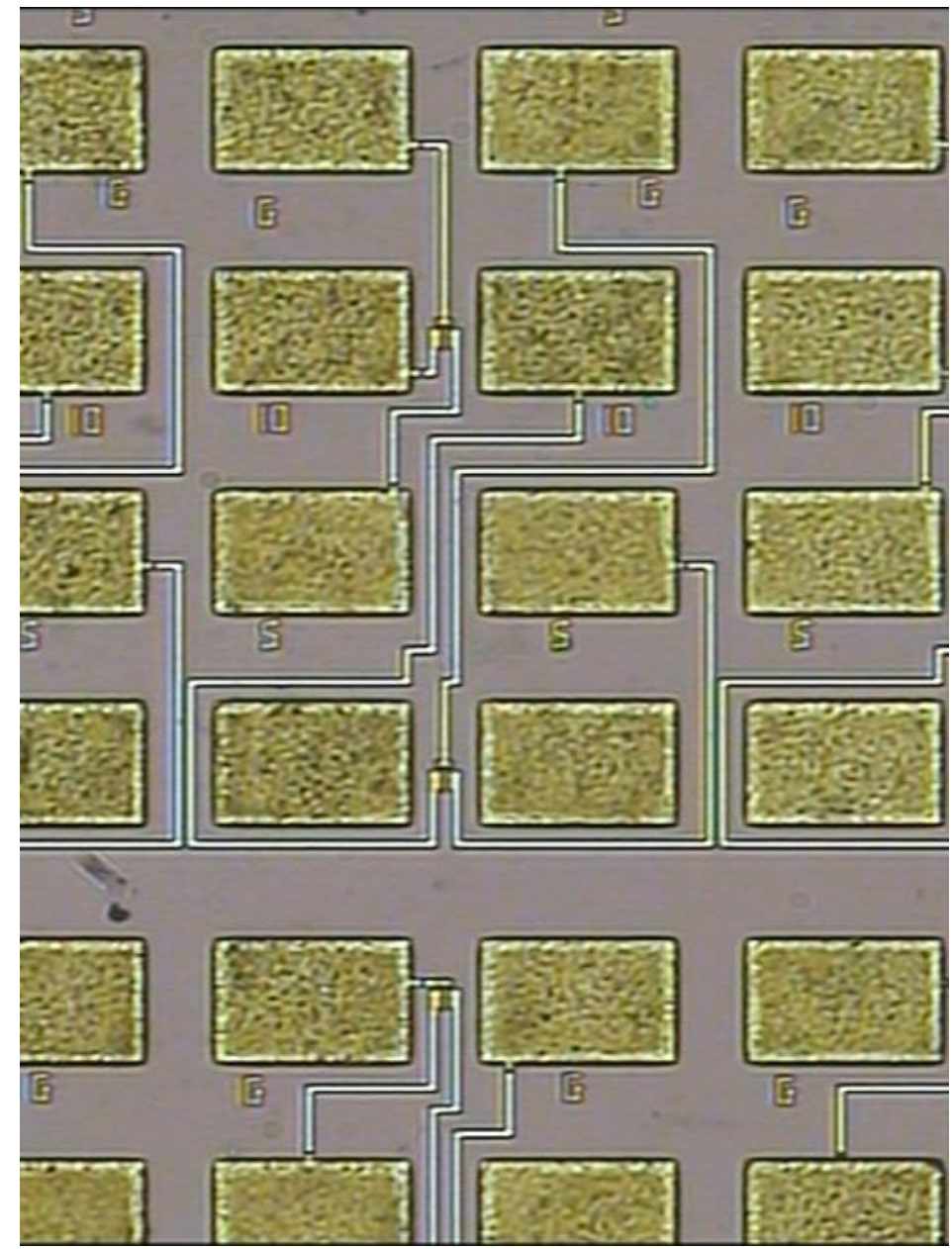

Fonte: Alves, 2017. 
Foram extraídas experimentalmente, curvas de corrente de dreno em função da tensão de porta e de dreno para cada um dos transistores. Com estas curvas foi possível calcular a razão $\left(\mathrm{L}_{\mathrm{LD}} / \mathrm{L}\right)_{\text {eff }}$ para os GC SOI MOSFETs através da equação 37 (PAVANELLO, 2000):

$$
\left(\frac{\mathrm{L}_{\mathrm{LD}}}{\mathrm{L}}\right)_{\mathrm{eff}}=1-\left(\frac{\mathrm{I}_{\mathrm{DS}}}{\mathrm{I}_{\mathrm{DS}, \mathrm{GC}}}\right)
$$

Foram obtidos os valores da relação $\mathrm{L}_{\mathrm{LD}} / \mathrm{L}$ para cada um dos transistores fabricados. Para cada grupo foram calculados os valores médios e desvio da relação $\left(\mathrm{L}_{\mathrm{LD}} / \mathrm{L}\right)$ eff. Os valores calculados para os GC SOI MOSFETs estão na Tabela 3.

Tabela 3 - Relação (LLD/L)eff dos GC SOI MOSFETs.

\begin{tabular}{cc}
\hline $\mathbf{L}_{\mathbf{L D}} / \mathbf{L}$ & $\left(\mathrm{L}_{\mathrm{LD}} / \mathrm{L}\right)_{\mathrm{eff}}$ \\
\hline $\mathbf{0 , 2 5 0}$ & $0,264 \pm 0,0200$ \\
\hline $\mathbf{0 , 3 7 5}$ & $0,393 \pm 0,0347$ \\
\hline
\end{tabular}

Fonte: Alves, 2017.

Inicialmente, foram extraídas as curvas de corrente de dreno (IDS) em função da tensão de porta $\left(\mathrm{V}_{\mathrm{GF}}\right)$, com tensão de dreno de $\mathrm{V}_{\mathrm{DS}}=25 \mathrm{mV}$ e passo de $1 \mathrm{mV}$, para as quatro razões $\mathrm{L}_{\mathrm{LD}} / \mathrm{L}$ dos dispositivos. A partir destas curvas foram extraídos o valor médio da corrente (IDS,mean) e o desvio padrão ( $\left.\sigma \mathrm{I}_{\mathrm{DS}}\right)$ para cada comprimento de canal. A Figura 19 mostra as curvas $I_{D S}$,mean em função de $V_{G F}$ e a Figura 20 mostra as curvas da corrente em escala logarítmica em função de $\mathrm{V}_{\mathrm{GF}}$, ambas para as razões $\mathrm{L}_{\mathrm{LD}} / \mathrm{L}$ de $0,0.25,0.375$ e 1 . 
Figura 19 - IDS,mean em função de $\mathrm{V}_{\mathrm{GF}}$ para os GC SOI MOSFETs com $\mathrm{V}_{\mathrm{DS}}=25 \mathrm{mV}$ e $\mathrm{L}_{\mathrm{LD}} / \mathrm{L}$ de $0,0.25,0.375$ e 1 .

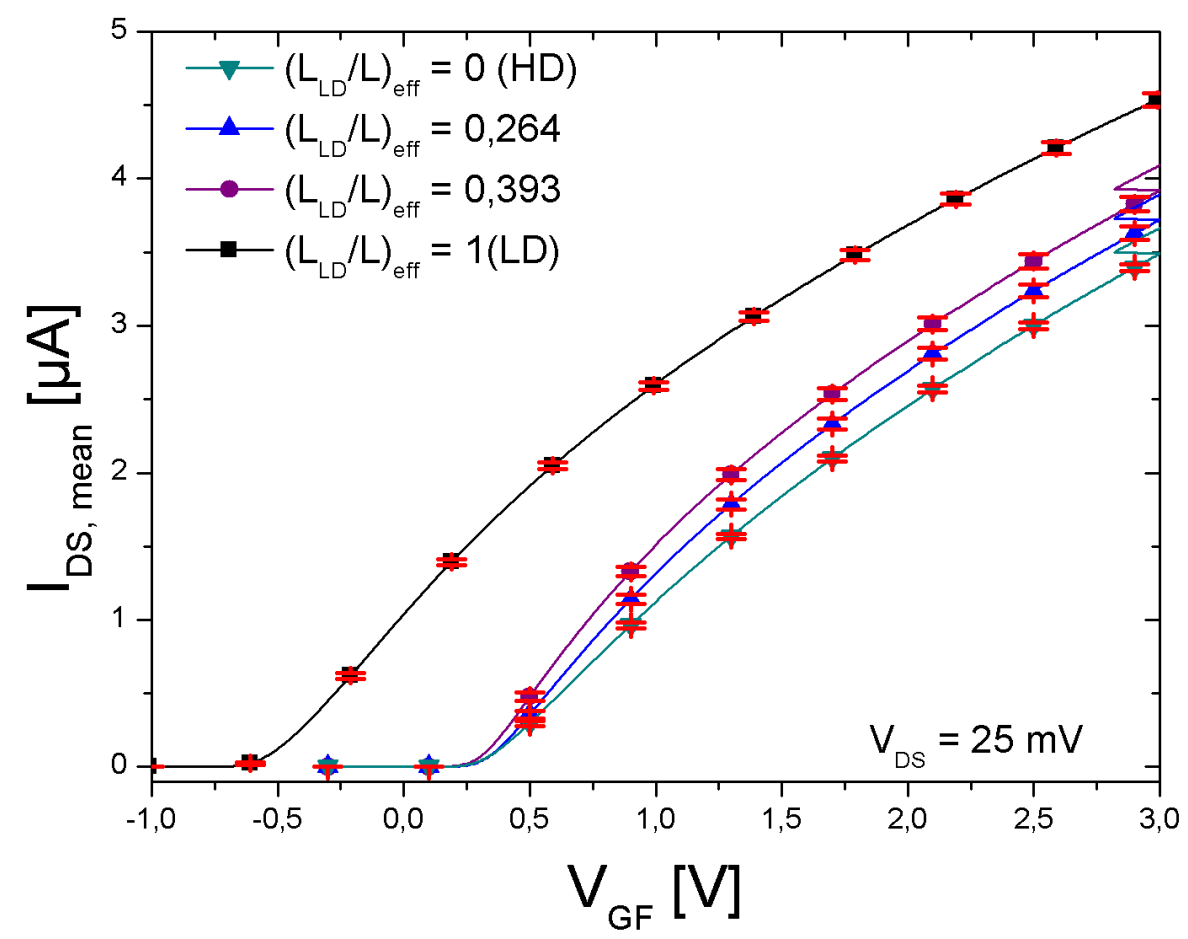

Fonte: Alves, 2017.

Figura 20 - $\mathrm{I}_{\mathrm{DS} \text {,mean }}$ em função de $\mathrm{V}_{\mathrm{GF}}$ em escala logarítmica para os GC SOI MOSFETs com $\mathrm{V}_{\mathrm{DS}}=25 \mathrm{mV}$ e $\mathrm{L}_{\mathrm{LD}} / \mathrm{L}$ de $0,0.25,0.375$ e 1 .

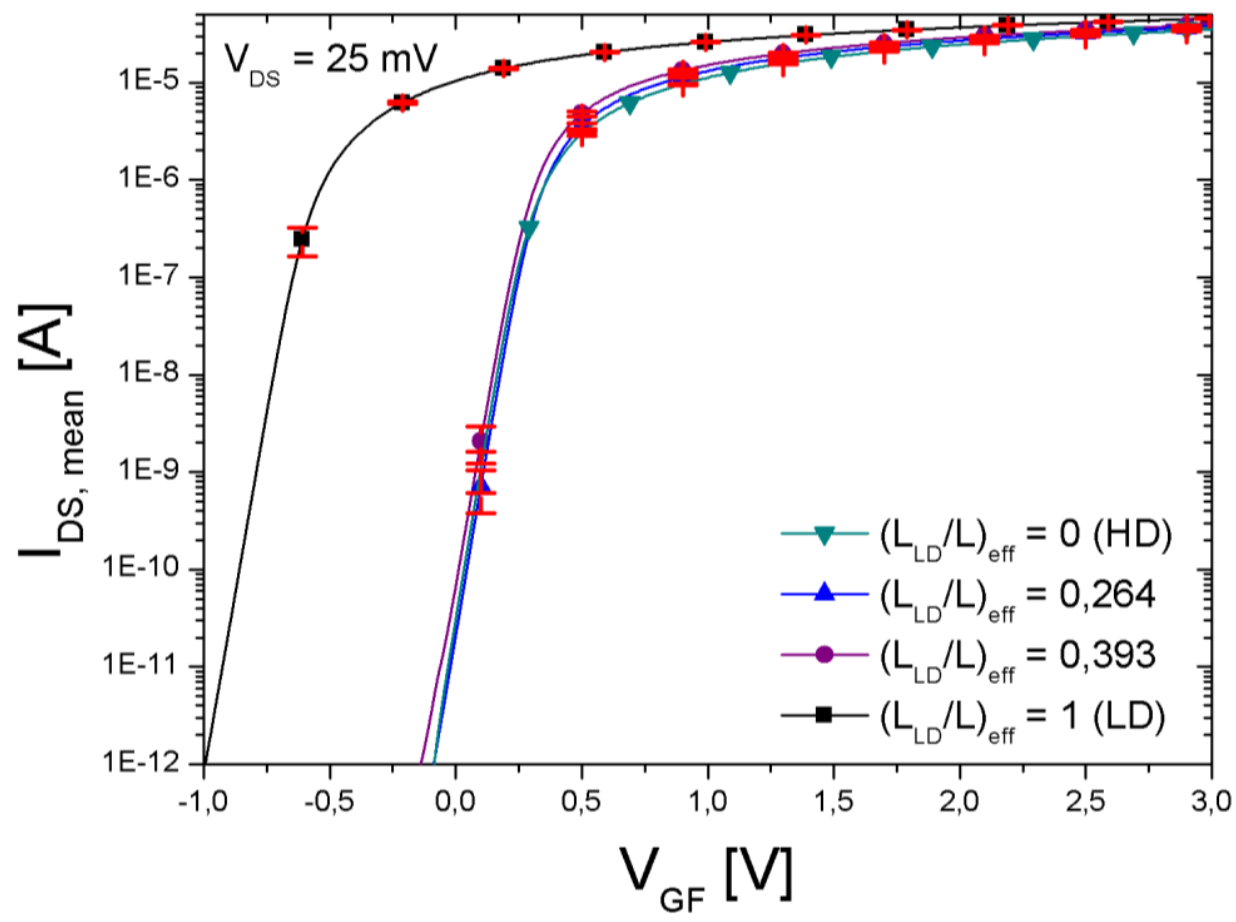

Fonte: Alves, 2017. 
Pode-se observar que conforme aumenta-se a razão $\mathrm{L}_{\mathrm{LD}} / \mathrm{L}$ há uma diminuição do comprimento de canal efetivo, o que faz com que a corrente aumente.

Ao colocar a corrente de dreno em escala logarítmica é possível extrair a inclinação de sublimiar (S) dos dispositivos. Novamente, foram extraídos os valores para cada transistor e, posteriormente, calculados o valor médio e desvio relativo. Os resultados encontrados estão na Tabela 4.

Tabela 4 - Inclinação de Sublimiar (S) dos SOI MOSFETs uniformemente dopado e GC SOI MOSFETs.

\begin{tabular}{cccc}
\hline $\mathbf{L}_{\mathbf{L D}} / \mathbf{L}$ & $\mathbf{( \mathbf { L } _ { \mathbf { L D } } / \mathbf { L } ) \text { eff }}$ & $\mathrm{S}_{\text {mean }}[\mathrm{mV} / \mathrm{dec}]$ & $\sigma \mathrm{S} / \mathrm{S}_{\text {mean }}[\%]$ \\
\hline $\mathbf{0 , 0 0 0}$ & - & 63,15 & 0,835 \\
\hline $\mathbf{0 , 2 5 0}$ & $\mathbf{0 , 2 6 4} \pm \mathbf{0 , 0 2 0 0}$ & 64,19 & 0,867 \\
\hline $\mathbf{0 , 3 7 5}$ & $\mathbf{0 , 3 9 3} \pm \mathbf{0 , 0 3 4 7}$ & 66,12 & 0,81 \\
\hline $\mathbf{1 , 0 0 0}$ & - & 68,16 & 0,785
\end{tabular}

Fonte: Alves, 2017.

Como a inclinação de sublimiar depende do fator de corpo, e sabe-se que este é menor para dispositivos SOI MOSFETs totalmente depletados, os valores encontrados estão conforme esperados (perto do valor teórico que é de $60 \mathrm{mV} / \mathrm{dec}$ ).

A tensão de limiar foi extraída pelo método do ponto máximo da segunda derivada da curva de corrente de dreno (IDs) em função da tensão de porta ( $\left.V_{G F}\right)$ (ORTIZ-CONDE, 2002), para cada um dos transistores.

$\mathrm{O}$ valor médio da tensão de limiar ( $\left.\mathrm{V}_{\text {thf,mean }}\right)$ e o desvio padrão relativo $\left(\sigma \mathrm{V}_{\text {thf }} / \mathrm{V}_{\text {thf,mean }}\right)$ calculados estão apresentados na Tabela 5.

Tabela 5 - Valor médio e desvio padrão relativo da tensão de limiar de transistores SOI MOSFETs uniformemente dopado e GC SOI MOSFETs.

\begin{tabular}{cccc}
\hline $\mathbf{L}_{\mathbf{L D}} / \mathbf{L}$ & $\mathbf{( \mathbf { L } _ { \mathbf { L D } } / \mathbf { L } ) \text { eff }}$ & $\mathrm{V}_{\text {thf,mean }}[\mathrm{mV}]$ & $\sigma \mathrm{V}_{\text {thf }} \mathrm{V}_{\text {thf,mean }}[\%]$ \\
\hline $\mathbf{0 , 0 0 0}$ & - & 104,89 & 6,15 \\
\hline $\mathbf{0 , 2 5 0}$ & $\mathbf{0 , 2 6 4} \pm \mathbf{0 , 0 2 0 0}$ & 127,53 & 6,21 \\
\hline $\mathbf{0 , 3 7 5}$ & $\mathbf{0 , 3 9 3} \pm \mathbf{0 , 0 3 4 7}$ & 105,26 & 6,86 \\
\hline $\mathbf{1 , 0 0 0}$ & - & $-596,50$ & 2,08
\end{tabular}

Fonte: Alves, 2017. 
Pode-se observar que, para os dispositivos uniformemente dopados $\left(\mathrm{L}_{\mathrm{LD}} / \mathrm{L}=0\right.$ e 1 , que representam os transistores fortemente e fracamente dopado, respectivamente), conforme a concentração de dopantes aumenta a sua tensão de limiar também aumenta, já que a mesma é diretamente proporcional às cargas de depleção. Pode-se observar um aumento do desvio relativo diretamente proporcional à concentração de dopantes. Conforme discutido em artigos anteriores, a variação da tensão de limiar é atribuída à variação na concentração de dopantes e o desvio aumenta com o aumento da concentração (CROON, 2002).

Para os dispositivos GC SOI MOSFETs pode ser observado que conforme aumenta-se a razão $\mathrm{L}_{\mathrm{LD}} / \mathrm{L}$ a tensão de limiar diminui, devido à diminuição do comprimento efetivo do canal e consequentemente uma diminuição da fração de cargas de depleção controladas pela porta do dispositivo. Os dispositivos GC SOI MOSFETs apresentam tensão de limiar próxima ao dispositivo uniformemente dopado de maior concentração de canal. Além disso, pode-se observar uma piora no desvio relativo da tensão de limiar, o que pode ser associado à diminuição do comprimento de canal conforme aumenta-se a razão $\mathrm{L}_{\mathrm{LD}} / \mathrm{L}$, conforme pode ser observado na Figura 21. Para comprimentos efetivos de canal menores, qualquer variação da posição da transição entre as regiões HD e LD torna-se mais significativa.

Figura $21-\sigma \mathrm{V}_{\text {thf }} / \mathrm{V}_{\text {thf,mean }}$ em função da relação $1 /\left(\mathrm{W} \times \mathrm{L}_{\mathrm{eff}}\right)^{1 / 2}$ para transistores GC SOI e suas razões $\mathrm{L}_{\mathrm{LD}} / \mathrm{L}$.

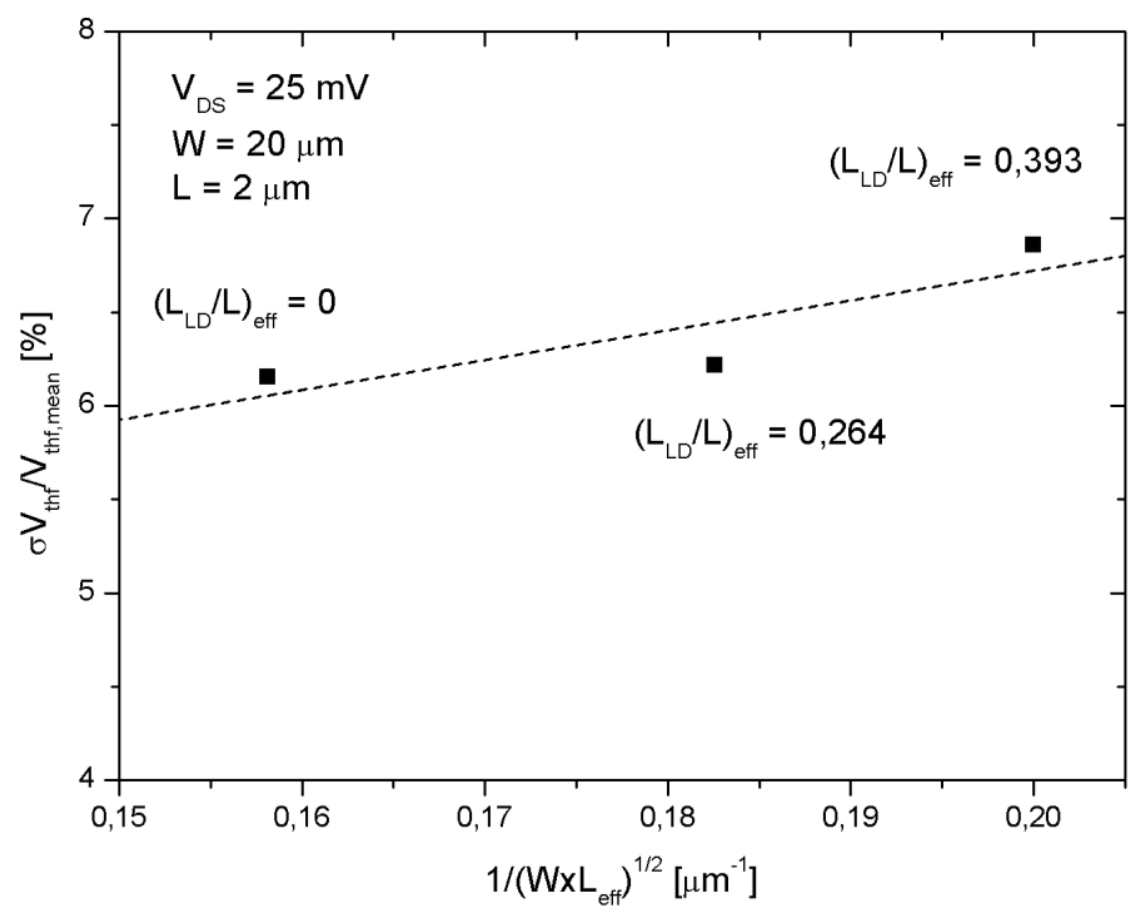

Fonte: Alves, 2017. 
Para analisar o descasamento da corrente de dreno, foram traçadas as curvas do desvio padrão relativo $\left(\sigma \mathrm{I}_{\mathrm{DS}} / \mathrm{I}_{\mathrm{DS} \text {,mean }}\right)$ em função da sobretensão de condução $\left(\mathrm{V}_{\mathrm{GT}}=\mathrm{V}_{\mathrm{GF}}-\mathrm{V}_{\text {thf }}\right)$, para que a variação da corrente de porta não seja dependente da tensão de limiar. Os resultados são apresentados na Figura 22, com polarização de dreno de $\mathrm{V}_{\mathrm{DS}}=25 \mathrm{mV}$, e na Figura 23 para $\mathrm{V}_{\mathrm{DS}}=1,5 \mathrm{~V}$.

Figura 22 - Desvio padrão relativo da corrente de dreno em função da sobretensão de condução com tensão de dreno $\mathrm{V}_{\mathrm{DS}}$ de $25 \mathrm{mV}$.

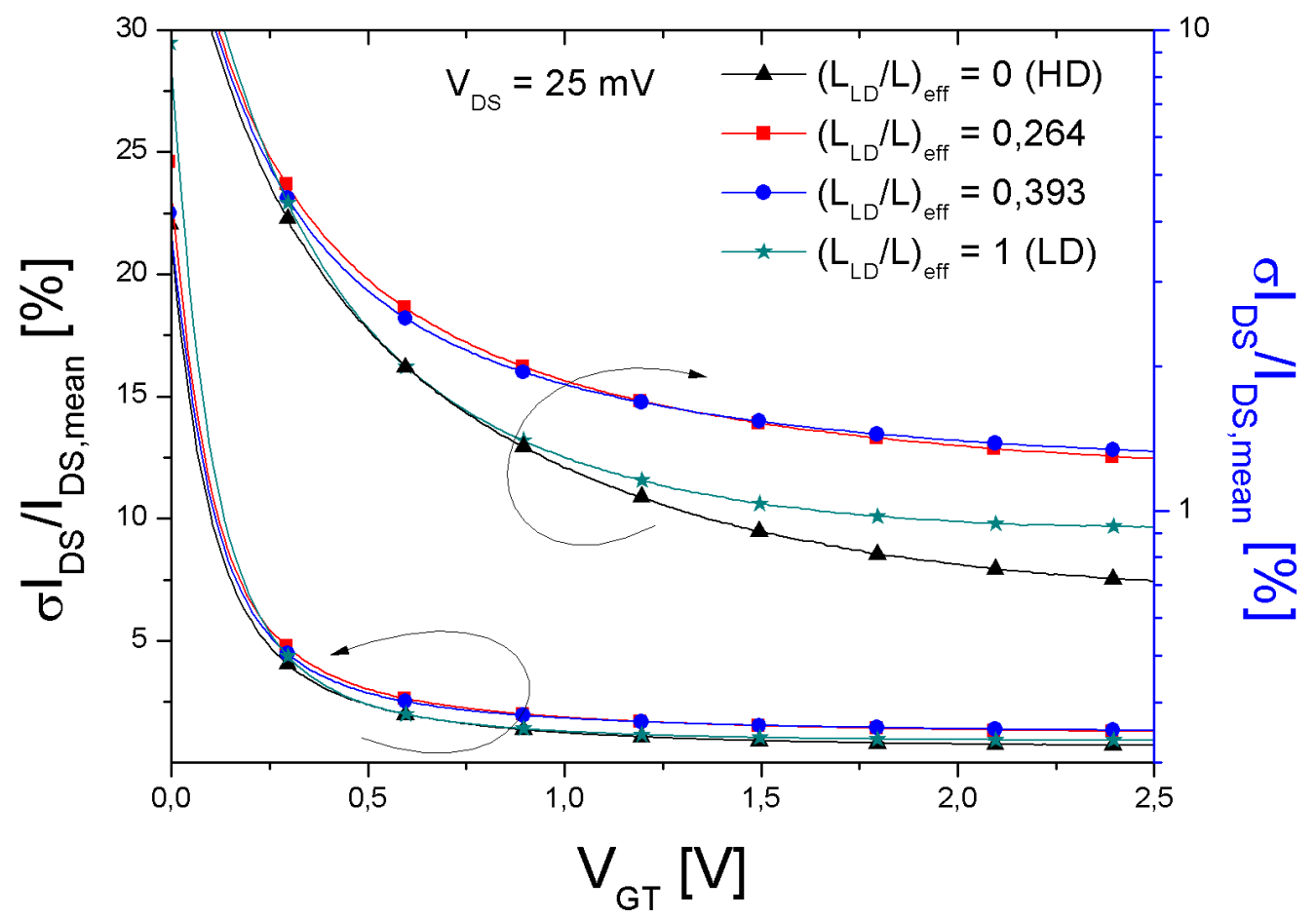

Fonte: Alves, 2017.

Figura 23 - Desvio padrão relativo da corrente de dreno em função da sobretensão de condução com tensão de dreno $\mathrm{V}_{\mathrm{DS}}$ de $1,5 \mathrm{~V}$. 


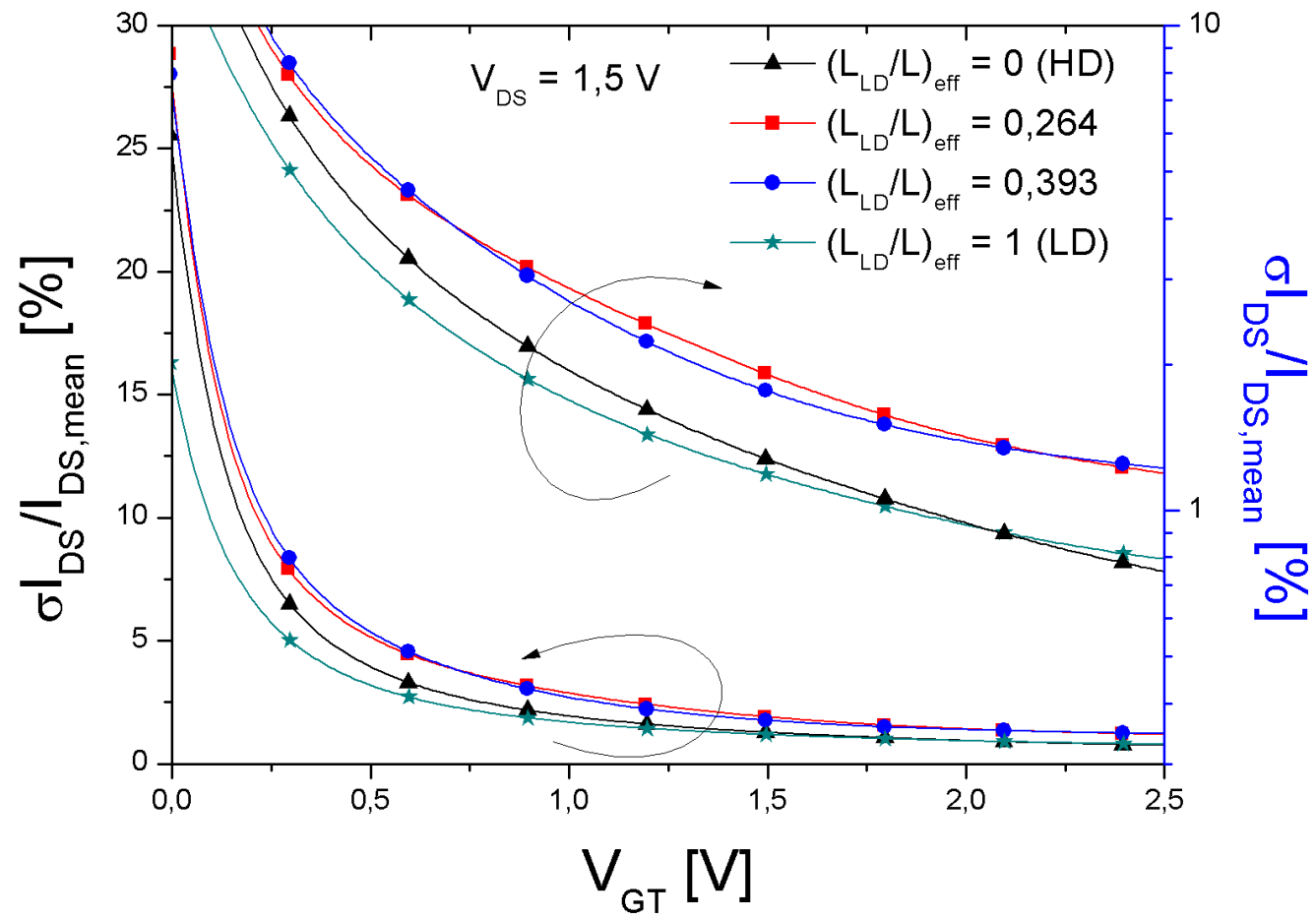

Fonte: Alves, 2017.

Pode-se observar que o descasamento da corrente de dreno é maior quando os dispositivos operam em inversão fraca (baixos valores de $\mathrm{V}_{\mathrm{GT}}$ ). Nesta condição de polarização, as características elétricas dos dispositivos sofrem grande influência do descasamento da tensão de limiar. Para altos valores da sobretensão de porta, o principal fator do descasamento da corrente de dreno é o descasamento do fator de corrente (VANCAILLIE, 2003). À medida que a tensão aplicada aumenta e os dispositivos passam a operar em inversão forte, o desvio relativo da corrente de dreno tende a um valor constante, próximo a $1 \%$, conforme pode ser visto com maior precisão através da escala logarítmica. Dispositivos GC SOI MOSFETs apresentam uma piora do casamento da corrente de dreno em comparação com dispositivos uniformemente dopados. Uma das causas para o aumento do descasamento está relacionada a imprecisões na definição de comprimento das regiões com diferentes concentrações de dopantes (SOUZA; FLANDRE, 2008). Entre os dispositivos uniformemente dopados, o aumento da concentração de dopantes provoca uma piora do casamento, conforme reportado na literatura (CROON, 2002).

As curvas da transcondutância média, obtidas a partir da derivada das curvas de corrente, são apresentadas na Figura 24 e Figura 25, para $\mathrm{V}_{\mathrm{DS}}=50 \mathrm{mV}$ e 1,5V, respectivamente. Pode-se notar, como reportado na literatura, que conforme aumentamos a 
razão $\mathrm{LLD}_{\mathrm{LD}} / \mathrm{L}$, com a redução do comprimento efetivo do canal, há um aumento da transcondutância em saturação.

Figura 24 - Transcondutância média em função da tensão de condução $\mathrm{V}_{\mathrm{DS}}$ de $25 \mathrm{mV}$.

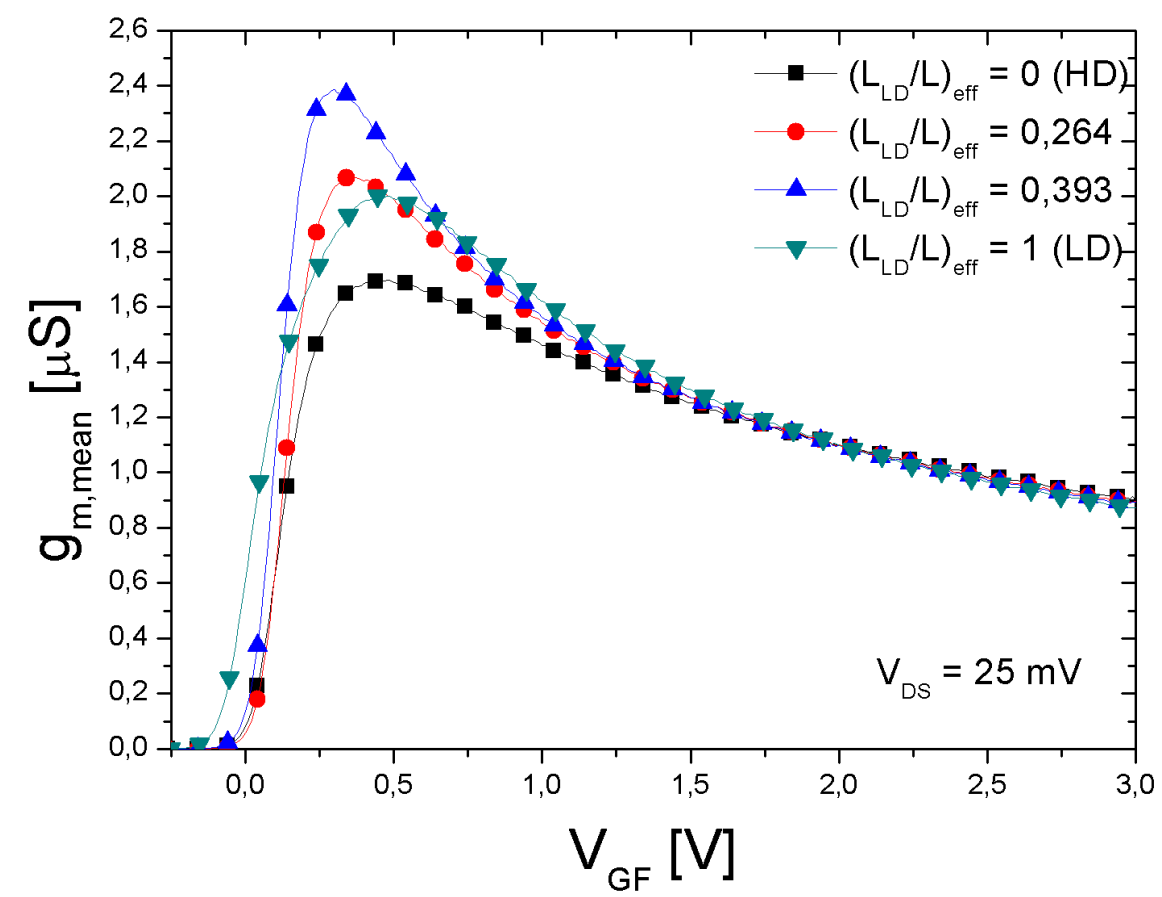

Fonte: Alves, 2017.

Figura 25 - Transcondutância média em função da tensão de condução com $V_{\text {DS }}$ de 1,5 V.

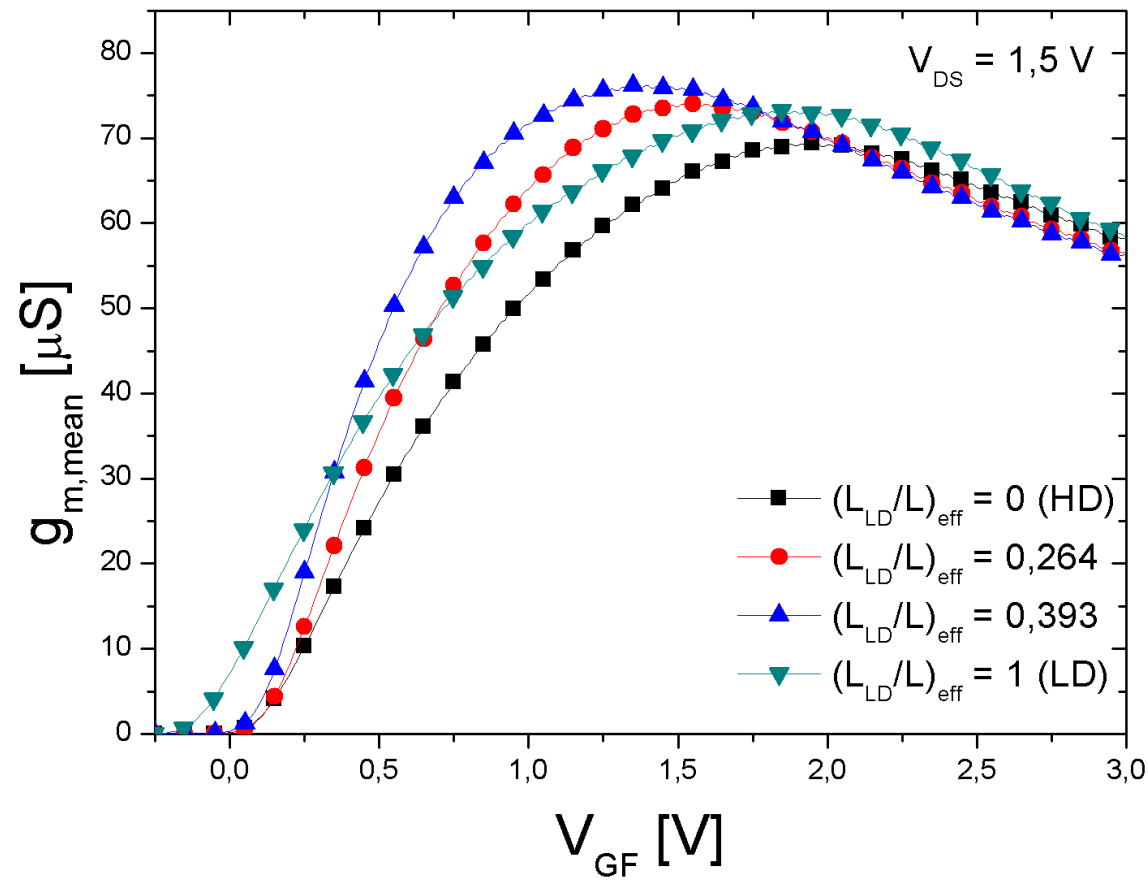

Fonte: Alves, 2017. 
Posteriormente, foram traçadas as curvas do desvio relativo da transcondutância $\left(\sigma g_{m} / g_{m}\right.$,mean $)$ em função da tensão de porta $\left(V_{G F}\right)$, conforme apresentado na Figura 26 e na Figura 27, obtidas com $\mathrm{V}_{\mathrm{DS}}$ de $25 \mathrm{mV}$ e $1,5 \mathrm{~V}$, respectivamente.

Figura 26 - Desvio padrão relativo da transcondutância em função da tensão de condução com tensão de dreno $\mathrm{V}_{\mathrm{DS}}$ de $25 \mathrm{mV}$.

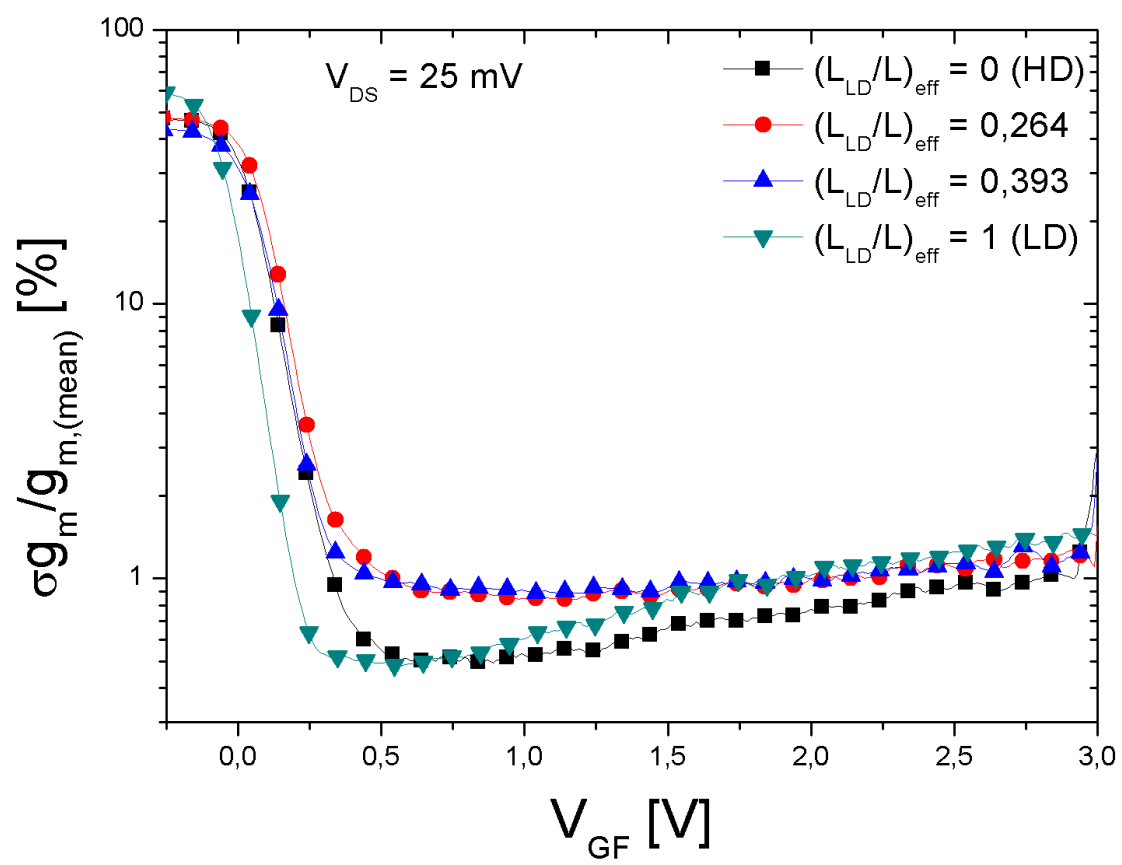

Fonte: Alves, 2017.

Figura 27 - Desvio padrão relativo da transcondutância em função da tensão de condução com tensão de dreno $\mathrm{V}_{\mathrm{DS}}$ de $1,5 \mathrm{~V}$.

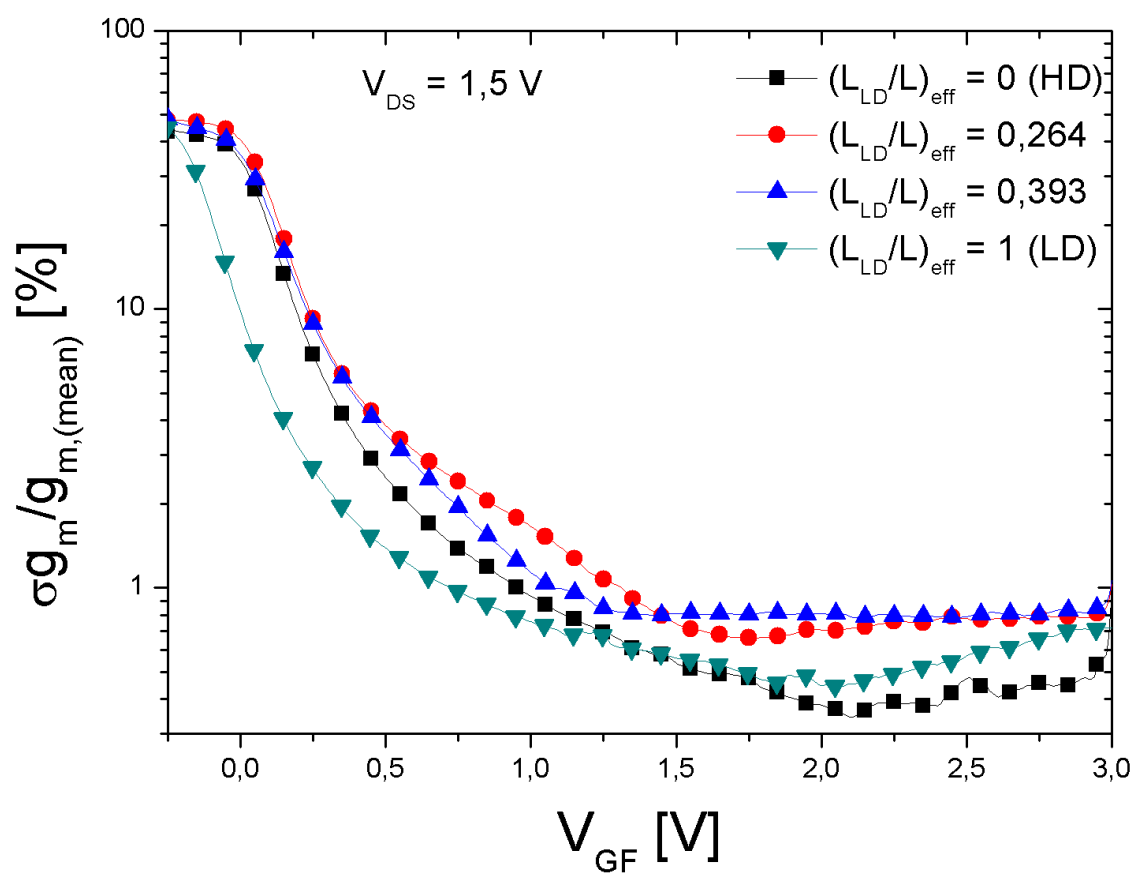

Fonte: Alves, 2017. 
Os dispositivos GC SOI MOSFETs apresentam uma piora do descasamento da transcondutância em relação aos dispositivos uniformemente dopados. A medida que a tensão aplicada aumenta, o desvio da transcondutância tende a um valor inferior a $1 \%$ para todos os dispositivos.

A partir dos resultados apresentados na Tabela 6 pode-se notar que apesar do desvio relativo da transcondutância para GC SOI MOSFETs serem maiores do que para o dispositivo uniformemente dopado, o desvio relativo da transcondutância é apenas ligeiramente maior enquanto que o valor da transcondutância média é ligeiramente maior.

Tabela 6 - Valor médio e desvio relativo da transcondutância média para GC SOI MOSFETs, extraído para $\mathrm{V}_{\mathrm{GT}}=200 \mathrm{mV}$ e $\mathrm{V}_{\mathrm{DS}}=1,5 \mathrm{~V}$.

\begin{tabular}{cccc}
\hline $\mathbf{L} \mathbf{L D} / \mathbf{L}$ & $\mathbf{( \mathbf { L } _ { \mathbf { L D } } / \mathbf { L } ) \text { eff }}$ & $\mathrm{g}_{\mathrm{m}, \text { mean }}[\mu \mathrm{S}]$ & $\sigma \mathrm{g}_{\mathrm{m}} / \mathrm{g}_{\mathrm{m}, \text { mean }}[\%]$ \\
\hline $\mathbf{0 , 0 0 0}$ & - & 139,87 & 3,95 \\
\hline $\mathbf{0 , 2 5 0}$ & $\mathbf{0 , 2 6 4} \pm \mathbf{0 , 0 2 0 0}$ & 199,24 & 4,38 \\
\hline $\mathbf{0 , 3 7 5}$ & $\mathbf{0 , 3 9 3} \pm \mathbf{0 , 0 3 4 7}$ & 258,07 & 4,59 \\
\hline
\end{tabular}

Fonte: Alves, 2017.

Foram extraídas as curvas da corrente de dreno $\left(I_{D}\right)$ em função da tensão de dreno $\left(\mathrm{V}_{\mathrm{DS}}\right)$ para dois diferentes valores de sobretensão de condução $\left(\mathrm{V}_{\mathrm{GT}}\right)$, de $200 \mathrm{mV}$ e $800 \mathrm{mV}$, apresentadas na Figura 28 e na Figura 29, respectivamente. 
Figura 28 - Corrente de dreno média (IDS,mean) em função de $\mathrm{V}_{\mathrm{DS}}$ para transistores SOI de canal uniformemente dopado e gradual polarizados com $\mathrm{V}_{\mathrm{GT}}=200 \mathrm{mV}$.

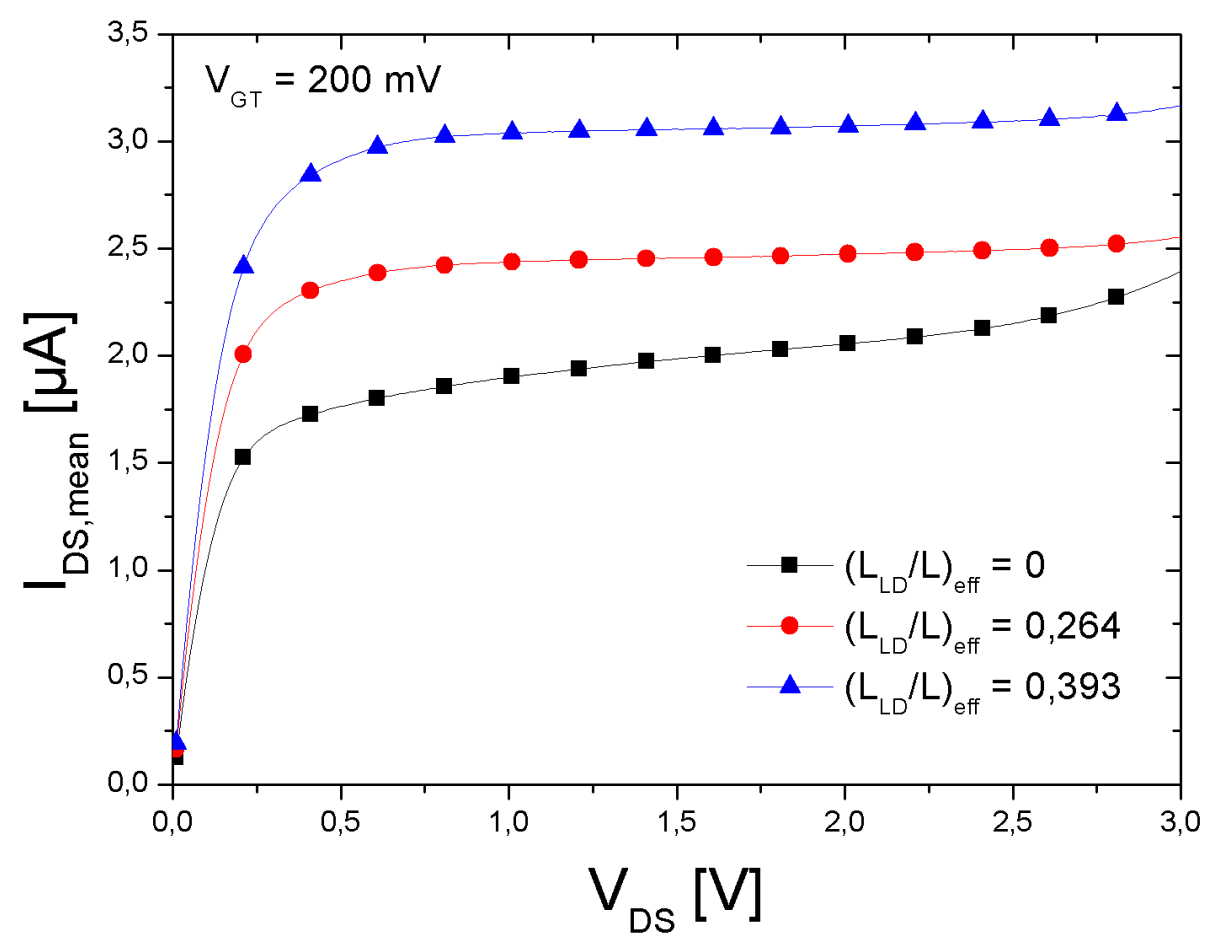

Fonte: Alves, 2017.

Figura 29 - Corrente de dreno média (IDS,mean) em função de $V_{D S}$ para transistores SOI de canal uniformemente dopado e gradual polarizados com $\mathrm{V}_{\mathrm{GT}}=800 \mathrm{mV}$.

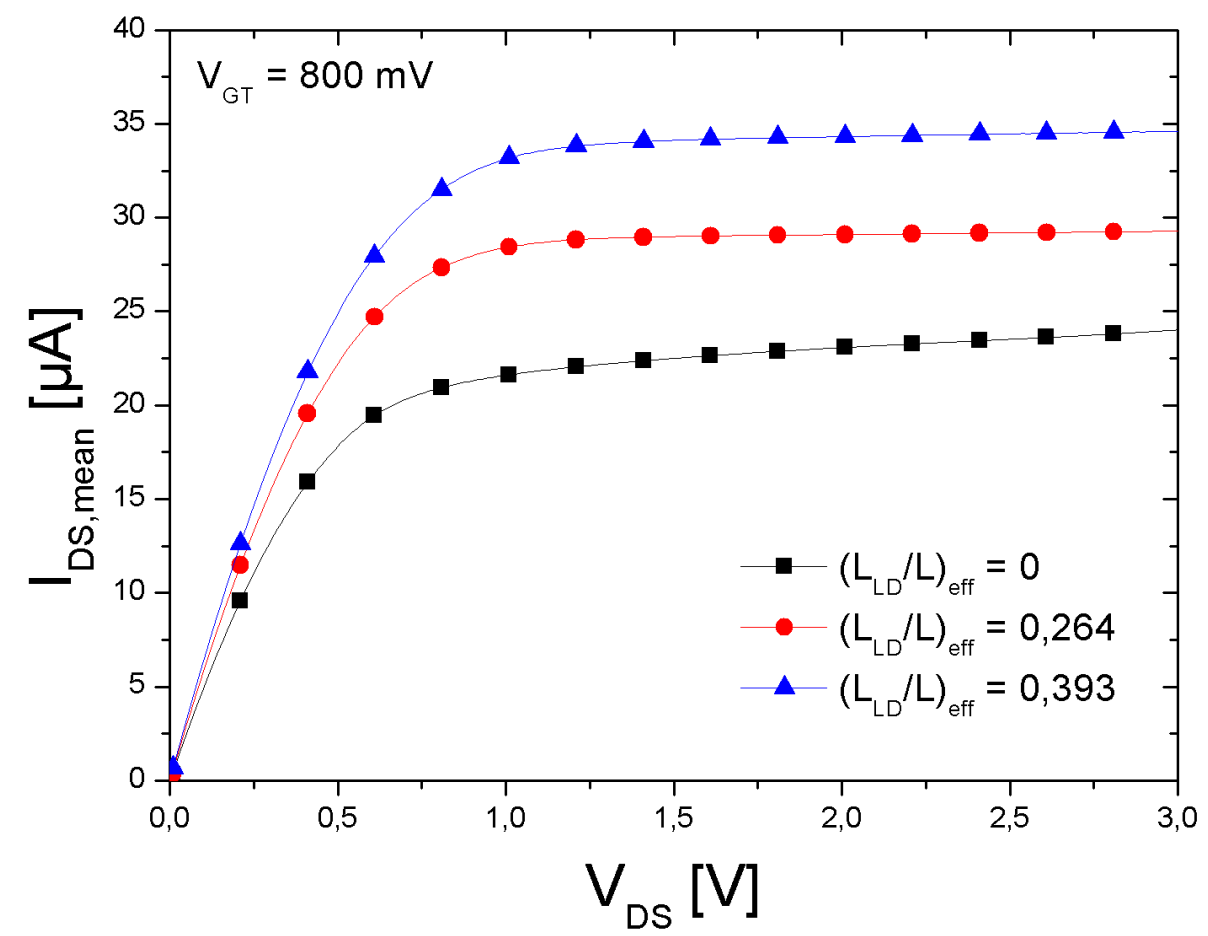

Fonte: Alves, 2017. 
Pode ser observado que conforme aumenta a razão $\mathrm{L}_{\mathrm{LD}} / \mathrm{L}$ a corrente aumenta, conforme reportado na literatura.

A partir destas curvas de corrente, posteriormente foi extraído o desvio padrão da corrente de dreno em função da tensão de dreno, conforme apresentado na Figura 30, para os dois valores de $\mathrm{V}_{\mathrm{GT}}$ medidos.

Figura 30 - Desvio padrão da corrente de dreno em função da tensão de dreno com sobretensão de condução de 200 e 800 mV.

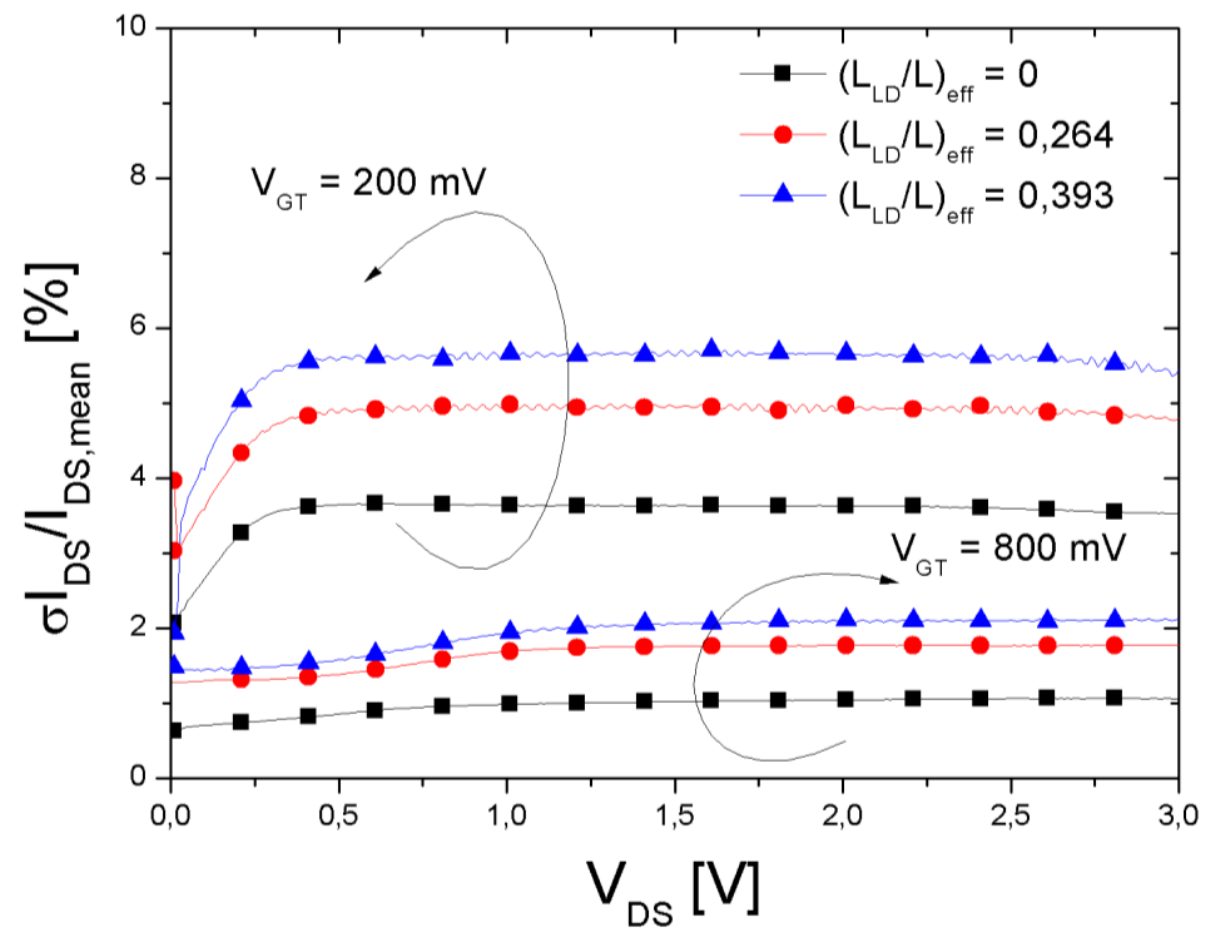

Fonte: Alves, 2017.

Pode-se observar que conforme aumentamos a razão $\mathrm{L}_{\mathrm{LD}} / \mathrm{L}$ o desvio da corrente tende a aumentar nos dispositivos GC SOI MOSFETs. Para dispositivos uniformemente dopados conforme aumenta-se a concentração de dopantes o desvio relativo diminui. Os dispositivos uniformemente dopados apresentam menor desvio relativo da corrente de dreno em relação aos dispositivos GC SOI MOSFETs, em torno de 3\% enquanto que os GC SOI MOSFETs possuem desvio relativo em torno de $5 \%$. O descasamento em saturação é praticamente constante independentemente do valor da tensão de dreno. Ao aplicarmos uma maior tensão de porta a influência da variação da tensão de limiar se torna menos significativa, diminuindo os desvios relativos. 
A partir das curvas de corrente, foram extraídos os valores de condutância de dreno para cada dispositivo. Conforme pode ser visto, a condutância de dreno de dispositivos GC SOI MOSFETs melhora conforme aumentamos a razão $\mathrm{L}_{\mathrm{LD}} / \mathrm{L}$, e apresenta uma grande melhora se comparado com dispositivos uniformemente dopado. Porém o desvio relativo da condutância de GC SOI MOSFETs é significativamente maior se comparado com dispositivos uniformemente dopados, conforme pode ser observado na Tabela 7.

Tabela 7 - Valor médio e desvio relativo da condutância de dreno ( $\left.\mathrm{g}_{\mathrm{D}}\right)$ para GC SOI MOSFETs, extraídos para $\mathrm{V}_{\mathrm{GT}}=200 \mathrm{mV}$ e $\mathrm{V}_{\mathrm{DS}}=1,5 \mathrm{~V}$.

\begin{tabular}{cccc}
\hline $\mathbf{L L D} / \mathbf{L}$ & $(\mathbf{L} \mathbf{L D} / \mathbf{L})$ eff & $\mathrm{g}_{\mathrm{D}, \text { mean }}[\mathrm{S}]$ & $\sigma \mathrm{g}_{\mathrm{D}} / \mathrm{g}_{\mathrm{D}, \text { mean }}[\%]$ \\
\hline $\mathbf{0 , 0 0 0}$ & - & $1,47.10^{-6}$ & 6,63 \\
\hline $\mathbf{0 , 2 5 0}$ & $\mathbf{0 , 2 6 4} \pm \mathbf{0 , 0 2 0 0}$ & $0,27.10^{-6}$ & 11,6 \\
\hline $\mathbf{0 , 3 7 5}$ & $\mathbf{0 , 3 9 3} \pm \mathbf{0 , 0 3 4 7}$ & $0,22.10^{-6}$ & 12,4 \\
\hline
\end{tabular}

Fonte: Alves, 2017.

A tensão pode ser obtida a partir da relação $\mathrm{I}_{\mathrm{DS}} / \mathrm{g}_{\mathrm{D}}$. Apesar do desvio relativo da corrente de dreno e da condutância de saída de dispositivos GC SOI MOSFETs serem maiores quando comparados com dispositivos uniformemente dopados, os altos valores da tensão Early fazem com que o desvio relativo seja pequeno, não passando de $3 \%$, como pode ser observado na Figura 31 e Figura 32. O ruído que pode ser observado na tensão Early está relacionado com o menor valor da condutância de saída. 
Figura 31 - Tensão Early em função da tensão de dreno com sobretensão de condução de 200 $\mathrm{mV}$.

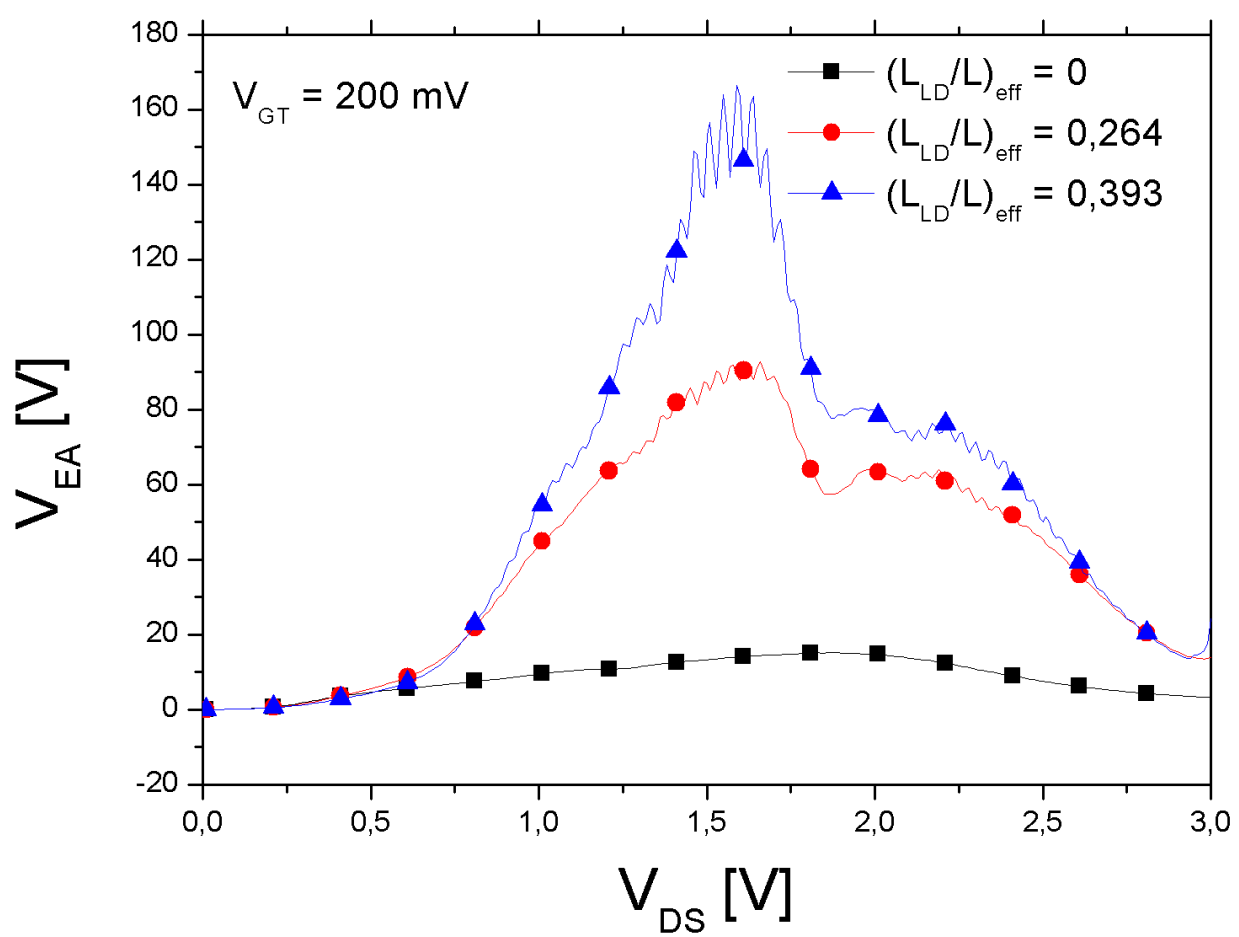

Fonte: Alves, 2017.

Figura 32 - Desvio da Tensão Early em função da tensão de dreno com sobretensão de condução de $200 \mathrm{mV}$.

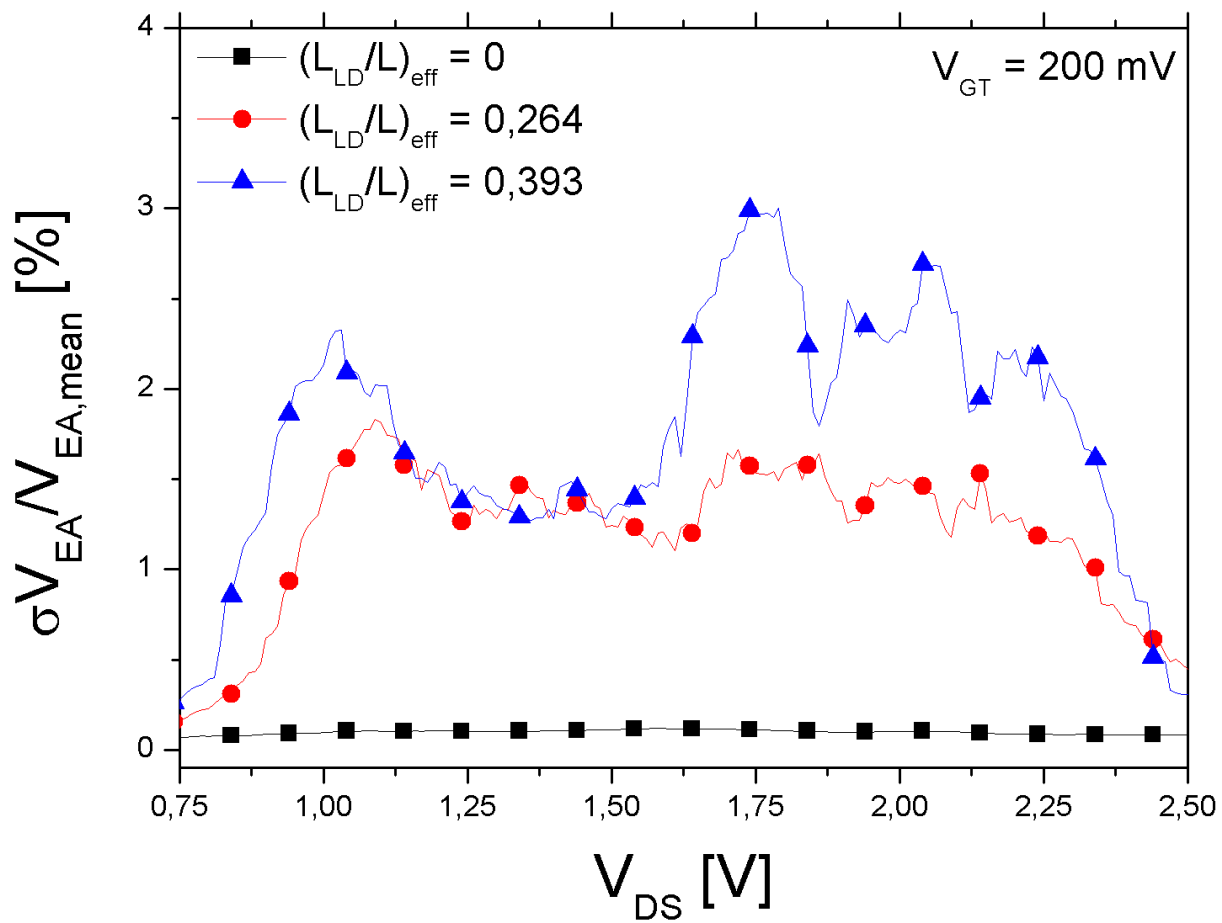

Fonte: Alves, 2017. 
Para a tensão de dreno de $1,5 \mathrm{~V}$ e sobretensão de porta de $200 \mathrm{mV}$ foram extraídos os valores médios da tensão Early conforme Tabela 8.

Tabela 8 - Tensão Early $\left(\mathrm{V}_{\mathrm{EA}}\right)$ dos transistores GC SOI MOSFETS e SOI uniformemente dopados, com $\mathrm{V}_{\mathrm{DS}}=1,5 \mathrm{~V}$ e $\mathrm{V}_{\mathrm{GT}}=200 \mathrm{mV}$.

\begin{tabular}{ccc}
\hline $\mathbf{L}_{\mathbf{L D}} / \mathbf{L}$ & $\left(\mathbf{L}_{\mathbf{L D}} / \mathbf{L}\right)$ eff & $\mathrm{V}_{\text {EA,mean }}[\mathrm{V}]$ \\
\hline $\mathbf{0 , 0 0 0}$ & - & 13,32 \\
\hline $\mathbf{0 , 2 5 0}$ & $\mathbf{0 , 2 6 4} \pm \mathbf{0 , 0 2 0 0}$ & 86,8 \\
\hline $\mathbf{0 , 3 7 5}$ & $\mathbf{0 , 3 9 3} \pm \mathbf{0 , 0 3 4 7}$ & 153,28
\end{tabular}

Fonte: Alves, $20 \overline{17 .}$

A presença de uma região fracamente dopada próxima ao dreno reduz a modulação do comprimento de canal, fazendo com que a condutância de dreno diminua em relação a transistores uniformemente dopados. Conforme aumentamos a razão $\mathrm{L}_{\mathrm{LD}} / \mathrm{L}$ a condutância de dreno diminui e, consequentemente, a tensão Early aumenta. O mesmo acontece se aumentarmos a concentração de dopantes.

Foi calculado a ganho de tensão $\left(\mathrm{A}_{\mathrm{V}}=\mathrm{g}_{\mathrm{m}} / \mathrm{g}_{\mathrm{D}}\right)$ para $\mathrm{V}_{\mathrm{GT}}=200 \mathrm{mV}$ e $\mathrm{V}_{\mathrm{DS}}=1,5 \mathrm{~V}$, para cada dispositivo. O ganho de tensão médio e o desvio relativo do ganho de tensão estão presentes na Tabela 9 .

Tabela 9 - Ganho de tensão $\left(\mathrm{A}_{V}\right)$ dos transistores GC SOI MOSFETS e SOI uniformemente dopados, com $\mathrm{V}_{\mathrm{DS}}=1,5 \mathrm{~V}$ e $\mathrm{V}_{\mathrm{GT}}=200 \mathrm{mV}$.

\begin{tabular}{cccc}
\hline $\mathbf{L}_{\mathbf{L D}} / \mathbf{L}$ & $\left.\mathbf{L}_{\mathbf{L D}} / \mathbf{L}\right)_{\text {eff }}$ & $\mathrm{A}_{\mathrm{V}, \text { mean }}[\mathrm{V} / \mathrm{V}]$ & $\sigma_{\mathrm{V},} / \mathrm{A}_{\mathrm{V}, \text { mean }}[\%]$ \\
\hline $\mathbf{0 , 0 0 0}$ & - & 95,99 & 4,11 \\
\hline $\mathbf{0 , 2 5 0}$ & $\mathbf{0 , 2 6 4} \pm \mathbf{0 , 0 2 0 0}$ & 744,75 & 9,93 \\
\hline $\mathbf{0 , 3 7 5}$ & $\mathbf{0 , 3 9 3} \pm \mathbf{0 , 0 3 4 7}$ & 1174,63 & 11,09 \\
\hline
\end{tabular}

Fonte: Alves, 2017.

Pode-se observar um grande aumento do ganho para dispositivos GC SOI MOSFETs e que este aumento se torna mais pronunciado à medida que a razão $\mathrm{L}_{\mathrm{LD}} / \mathrm{L}$ é aumentada. Entretanto, uma piora do descasamento relativo é observada. Os resultados obtidos para o desvio relativo do ganho de tensão são comparáveis aos valores de desvio relativo obtidos para a condutância de dreno, mostrando a alta influência do parâmetro $\mathrm{g}_{\mathrm{D}}$ no ganho de tensão. 


\section{RESULTADOS SIMULADOS}

Com o objetivo de avaliar isoladamente a influência da variação da concentração de dopantes e do comprimento da região fortemente e fracamente dopadas sobre as características elétricas de transistores GC SOI, foram realizadas simulações numéricas bidimensionais. Para estas simulações, foi utilizado o simulador Sentaurus, da Synopsys (SYNOPSYS, 2010). Foram consideradas as mesmas espessuras dos transistores fabricados, considerando dois grupos de dispositivos:

a) Transistores GC SOI com comprimento de canal de $2 \mu \mathrm{m}, \mathrm{N}_{\mathrm{AH}}=5 \times 10^{16} \mathrm{~cm}^{-3}, \mathrm{~N}_{\mathrm{AL}}=$ $1 \times 10^{15} \mathrm{~cm}^{-3}$, com razões $\mathrm{L}_{\mathrm{LD}} / \mathrm{L}$ médias de 0,$2 ; 0,3 ; 0,4$ e 0,5 , provocando variações no comprimento efetivo de canal de $\pm 30 \mathrm{~nm}$ (SOUZA; FLANDRE, 2008).

b) Transistores GC SOI com comprimento de canal de $2 \mu \mathrm{m}$, com razões $\mathrm{L}_{\mathrm{LD}} / \mathrm{L}$ de 0,$2 ; 0,3$; 0,4 e $0,5, \mathrm{~N}_{\mathrm{AL}}=1 \times 10^{15} \mathrm{~cm}^{-3}, \mathrm{~N}_{\mathrm{AH}}=5 \times 10^{16} \mathrm{~cm}^{-3} \pm 10 \%$.

Em todos os dispositivos, foi considerada transição abrupta entre as regiões fortemente e fracamente dopadas do canal. Os parâmetros de modelo foram ajustados com dados experimentais de transistores da mesma tecnologia. Os modelos utilizados nas simulações numéricas, responsáveis pela inclusão dos fenômenos físicos necessários para a adequada simulação dos dispositivos são os seguintes (ASSALTI, 2015):

a) PhuMob (Philips Unified Mobility Model): modelo proposto por Klaassen que considera as mobilidades dos portadores majoritários e minoritários de forma conjunta, contabilizando os mecanismos de espalhamento sobre a mobilidade, tais como espalhamento por impurezas ionizadas e portador-portador, além da dependência da mobilidade com a temperatura;

b) Enormal: modelo proposto por Lombardi que considera a influência do campo elétrico transversal sobre a mobilidade dos portadores, provocando uma maior interação dos portadores com a interface óxido-silício, aumentando a degradação da mobilidade em razão da rugosidade da superfície e dos mecanismos de espalhamento por fônons superficiais acústicos;

c) HighFieldSaturation: modelo proposto por Canali que contabiliza a influência do campo elétrico lateral sobre a mobilidade, uma vez que, devido à velocidade de saturação dos portadores, a mobilidade não é mais proporcional ao campo elétrico. 
d) SRH (Shockley-Read-Hall): modelo que considera a geração-recombinação de portadores. Quando utilizado com o sub-modelo DopingDep, é levada em conta a dependência do tempo de vida dos portadores com a concentração de dopantes, de acordo com o modelo Scharfetter;

e) Avalanche: modelo proposto por Van Overstraeten e De Man que insere os efeitos da ionização por impacto no comportamento elétrico do transistor;

f) Auger: recombinação Auger é um fenômeno físico onde um átomo neutro libera um elétron de sua camada eletrônica, dando origem a uma lacuna que é ocupada por outro elétron proveniente das camadas eletrônicas externas. Esta transição é acompanhada pela ejeção de um elétron com energia cinética;

g) BandGapNarrowing (OldSlotboom): considera o estreitamento da largura da faixa proibida.

No Apêndice B são representados os arquivos de simulação de um transistor GC SOI.

\subsection{VARIAÇÃO DO COMPRIMENTO EFETIVO DE CANAL}

No primeiro grupo de dispositivos simulados, as concentrações de dopantes das duas regiões do canal foram mantidas constantes e foi promovida uma variação na posição da transição de concentração de dopantes de $30 \mathrm{~nm}$.

A Tabela 10 apresenta o percentual de variação de LHD imposto nas simulações para cada relação $\mathrm{L}_{\mathrm{LD}} / \mathrm{L}$.

Tabela 10 - Percentual de variação de $\pm 30 \mathrm{~nm}$ em LHD.

\begin{tabular}{ccc}
\hline $\mathbf{L}_{\mathbf{L D}} / \mathbf{L}$ & $\mathrm{L}_{\mathrm{HD}}[\mu \mathrm{m}]$ & $\sigma \mathrm{L}_{\mathrm{HD}} / \mathrm{L}_{\mathrm{HD}}[\%]$ \\
\hline $\mathbf{0 , 2}$ & $1,57-1,63$ & 1,875 \\
\hline $\mathbf{0 , 3}$ & $1,37-1,43$ & 2,143 \\
\hline $\mathbf{0 , 4}$ & $1,17-1,23$ & 2,500 \\
\hline $\mathbf{0 , 5}$ & $0,97-1,03$ & 3,000
\end{tabular}

Fonte: Alves, $20 \overline{17 .}$ 
Primeiramente foram simuladas as curvas de corrente de dreno em função da tensão de porta com passo de $1 \mathrm{mV}$. A Figura 33 apresenta os resultados da corrente média obtidos usando uma tensão de polarização de dreno de $50 \mathrm{mV}$.

Figura 33 - Curva $\mathrm{I}_{\mathrm{DS} \text {,mean }}$ em função de $\mathrm{V}_{\mathrm{GT}}$ para os GC SOI MOSFETs com $\mathrm{L}_{\mathrm{HD}}$ variando \pm $30 \mathrm{~nm}, \mathrm{~V}_{\mathrm{DS}}=50 \mathrm{mV}$ e $\mathrm{L}_{\mathrm{LD}} / \mathrm{L}$ de $0.2,0.3,0.4,0.5$.

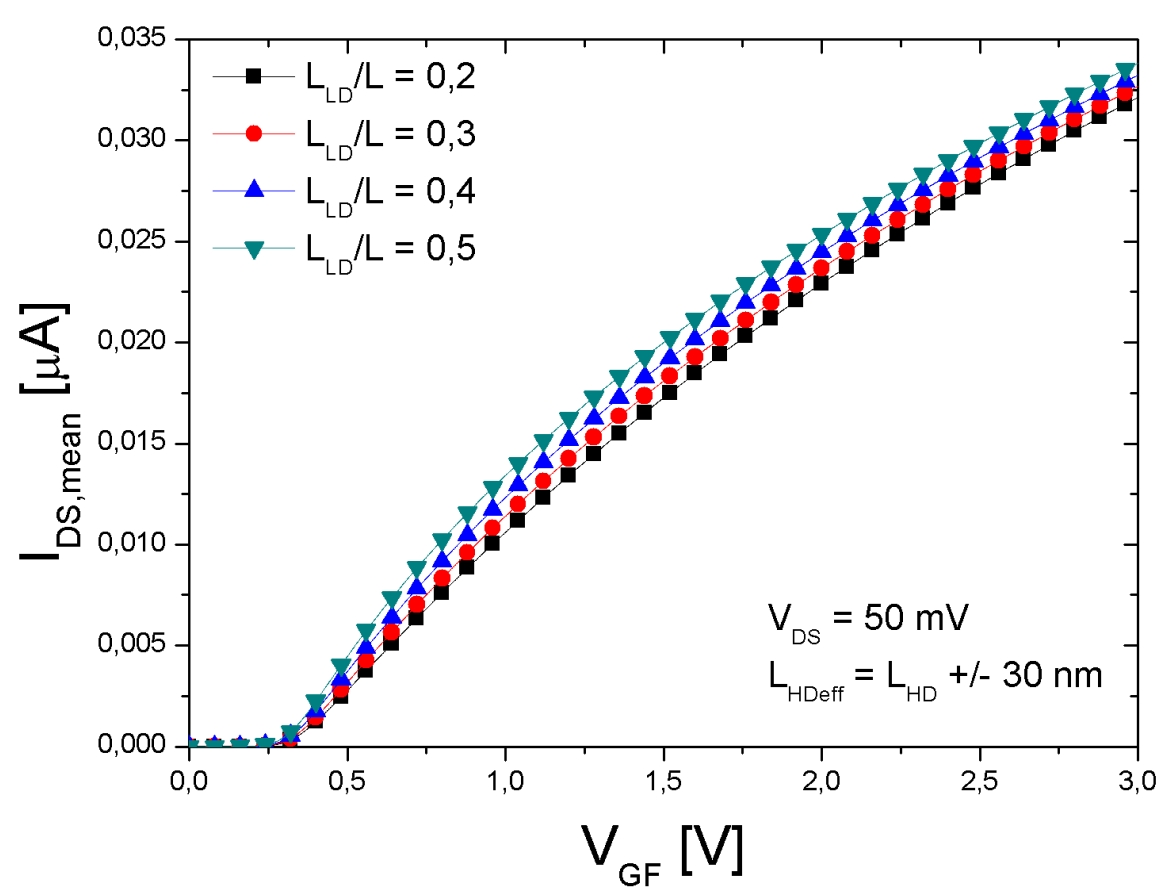

Fonte: Alves, 2017.

Pode ser observado um aumento da corrente conforme aumenta-se a razão $\mathrm{L}_{\mathrm{LD}} / \mathrm{L}$, conforme esperado e discutido previamente. Foram extraídas também curvas IDS em função de $\mathrm{V}_{\mathrm{GF}}$ para as tensões de condução de $1,5 \mathrm{~V}$ e $3 \mathrm{~V}$, para análise da operação em saturação. Os resultados obtidos para a corrente média são apresentados na Figura 34 e Figura 35, respectivamente. Através destas figuras pode ser observado um aumento da corrente de dreno conforme aumentamos a tensão de polarização. Como exemplo usando a razão $\mathrm{L}_{\mathrm{LD}} / \mathrm{L}=0,2$ em $\mathrm{V}_{\mathrm{GF}}=2,7 \mathrm{~V}$ tem-se uma corrente de dreno de aproximadamente $0,6 \mu \mathrm{A}$ para $\mathrm{V}_{\mathrm{DS}}=1,5 \mathrm{~V}$ e aproximadamente $0,7 \mu \mathrm{A}$ para $\mathrm{V}_{\mathrm{DS}}=3 \mathrm{~V}$. 
Figura 34 - Curva IDS,mean em função de $\mathrm{V}_{\mathrm{GT}}$ para os GC SOI MOSFETs com $\mathrm{L}_{\mathrm{HD}}$ variando \pm $30 \mathrm{~nm}, \mathrm{~V}_{\mathrm{DS}}=1,5 \mathrm{~V}$ e $\mathrm{L}_{\mathrm{LD}} / \mathrm{L}$ de $0.2,0.3,0.4,0.5$.

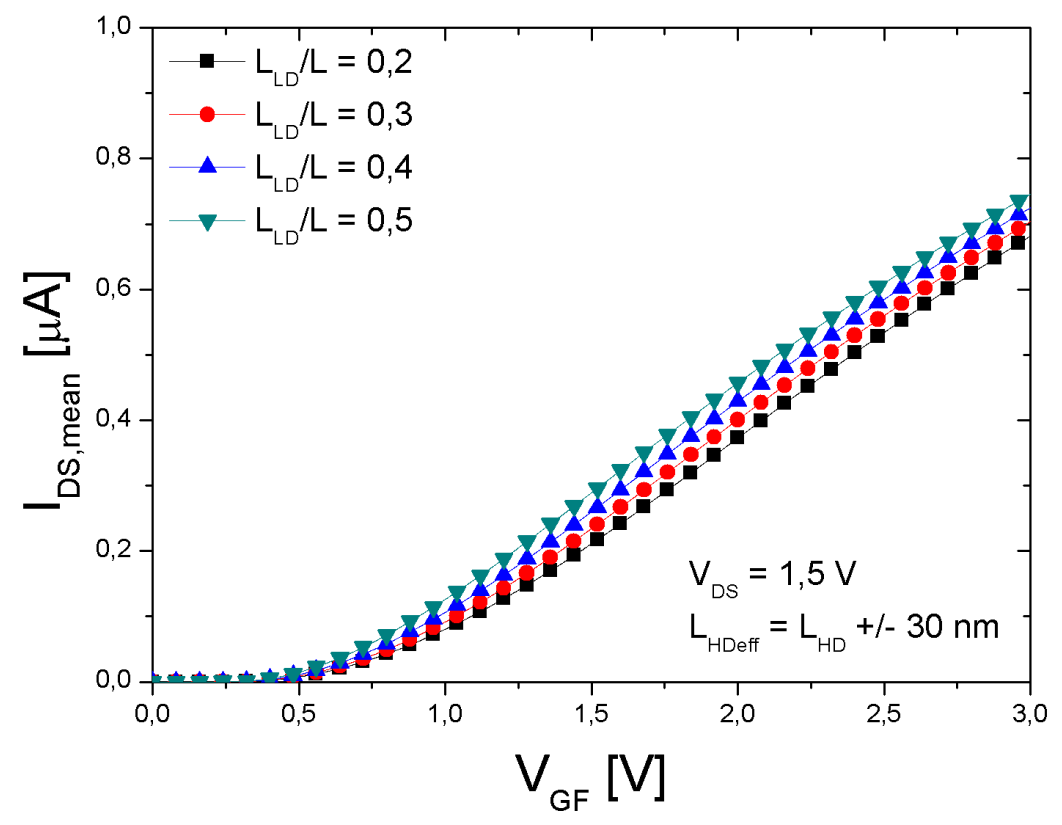

Fonte: Alves, 2017.

Figura 35 - Curva IDS,mean em função de $\mathrm{V}_{\mathrm{GT}}$ para os GC SOI MOSFETs com $\mathrm{L}_{\mathrm{HD}}$ variando \pm $30 \mathrm{~nm}, \mathrm{~V}_{\mathrm{DS}}=3 \mathrm{~V}$ e $\mathrm{L}_{\mathrm{LD}} / \mathrm{L}$ de $0.2,0.3,0.4,0.5$.

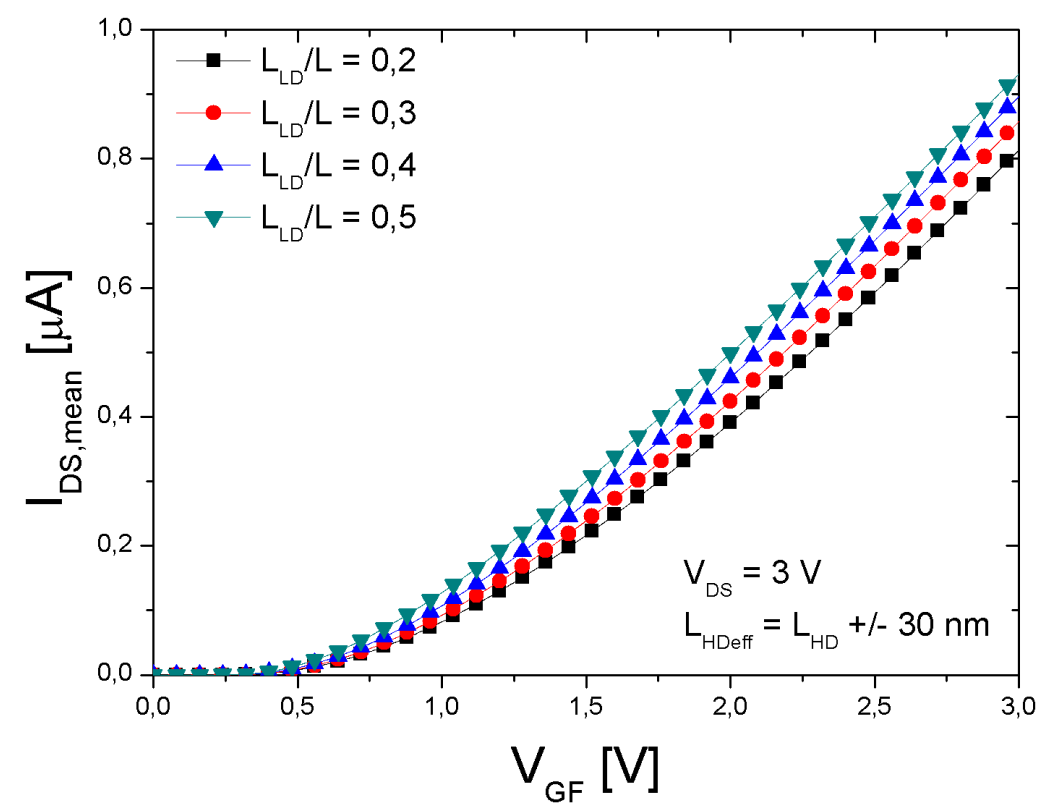

Fonte: Alves, 2017. 
Através das curvas $\mathrm{I}_{\mathrm{DS}}$ em função de $\mathrm{V}_{\mathrm{GF}}$ com $\mathrm{V}_{\mathrm{DS}}=50 \mathrm{mV}$, foram extraídos os valores da tensão de limiar para cada razão $\mathrm{L}_{\mathrm{LD}} / \mathrm{L}$ e suas variações, conforme apresentado na Tabela 11.

Tabela 11 - Valor médio e desvio relativo da tensão de limiar de GC SOI MOSFETs com LHD variando $\pm 30 \mathrm{~nm}, \mathrm{~V}_{\mathrm{DS}}=50 \mathrm{mV}$ e $\mathrm{L}_{\mathrm{LD}} / \mathrm{L}$ de $0.2,0.3,0.4,0.5$.

\begin{tabular}{ccc}
\hline $\mathbf{L L D}_{\mathbf{L}} \mathbf{L}$ & $\mathrm{V}_{\text {thf,mean }}[\mathrm{V}]$ & $\sigma \mathrm{V}_{\text {thf }} / \mathrm{V}_{\text {thf,mean }}[\%]$ \\
\hline $\mathbf{0 , 2}$ & 0,319 & 0,181 \\
\hline $\mathbf{0 , 3}$ & 0,316 & 0,182 \\
\hline $\mathbf{0 , 4}$ & 0,311 & 0,321 \\
\hline $\mathbf{0 , 5}$ & 0,304 & 0,328
\end{tabular}

Fonte: Alves, $20 \overline{17 .}$

Pode ser observada uma diminuição da tensão de limiar média conforme aumentamos a razão $\mathrm{L}_{\mathrm{LD}} / \mathrm{L}$, devido à redução do comprimento efetivo do canal, que diminui a quantidade de cargas de depleção controladas pela porta do transistor. Há também uma piora do desvio relativo da tensão de limiar conforme aumentamos a razão $\mathrm{L}_{\mathrm{LD}} / \mathrm{L}$. Estes resultados apresentam a mesma tendência vista para os resultados experimentais. Entretanto, o desvio relativo da tensão de limiar é menor do que o desvio relativo imposto no comprimento efetivo de canal. Como exemplo, pode-se citar o transistor com $\mathrm{L}_{\mathrm{LD}} / \mathrm{L}=0,3$, no qual foi imposto um desvio relativo de $2,143 \%$ no comprimento da região fortemente dopado, resultando em um desvio relativo da tensão de limiar de $0,182 \%$. Desta forma, pode-se concluir que eventuais desvios na definição da região fortemente dopada têm pouco impacto sobre a tensão de limiar do dispositivo.

A inclinação de sublimiar também foi extraída a partir das curvas de corrente de dreno em função da tensão de porta. Os valores médios e seus desvios relativos são apresentados na Tabela 12. 
Tabela 12 - Valor médio e desvio relativo da inclinação de sublimiar de GC SOI MOSFETs com $\mathrm{L}_{\mathrm{HD}}$ variando $\pm 30 \mathrm{~nm}, \mathrm{~V}_{\mathrm{DS}}=50 \mathrm{mV}$ e $\mathrm{L}_{\mathrm{LD}} / \mathrm{L}$ de $0.2,0.3,0.4,0.5$.

\begin{tabular}{ccc}
\hline $\mathbf{L} \mathbf{L D} / \mathbf{L}$ & $\mathrm{S}_{\text {mean }}[\mathrm{mV} / \mathrm{dec}]$ & $\sigma \mathrm{S} / \mathrm{S}_{\text {mean }}[\%]$ \\
\hline $\mathbf{0 , 2}$ & 65,7 & 0 \\
\hline $\mathbf{0 , 3}$ & 66,2 & 0 \\
\hline $\mathbf{0 , 4}$ & 67,2 & 0 \\
\hline $\mathbf{0 , 5}$ & 68,3 & 0 \\
\hline
\end{tabular}

Fonte: Alves, $20 \overline{17 .}$

Pode ser observado um leve aumento da inclinação de sublimiar conforme aumentamos a razão $\mathrm{L}_{\mathrm{LD}} / \mathrm{L}$, porém os resultados estão próximos do valor teórico de 60 $\mathrm{mV} / \mathrm{dec}$, mostrando que não há uma degradação importante para dispositivos com menor comprimento de canal efetivo. Pode-se notar que apesar da variação aplicada no comprimento efetivo de canal a inclinação de sublimiar é basicamente constate, o que é denotado pelo desvio relativo nulo.

Foi traçada também a curva do desvio relativo da corrente de dreno em função da sobretensão de condução com polarização de dreno de $50 \mathrm{mV}, 1.5 \mathrm{~V}$ e $3 \mathrm{~V}$, apresentadas na Figura 36. 
Figura 36 - Desvio relativo da corrente de dreno em função da sobretensão de condução de com $\mathrm{V}_{\mathrm{DS}}=50 \mathrm{mV}(\mathrm{A}), 1,5 \mathrm{~V}$ (B) e $3 \mathrm{~V}(\mathrm{C})$, com $\mathrm{L}_{\mathrm{HD}}$ variando $\pm 30 \mathrm{~nm}$.
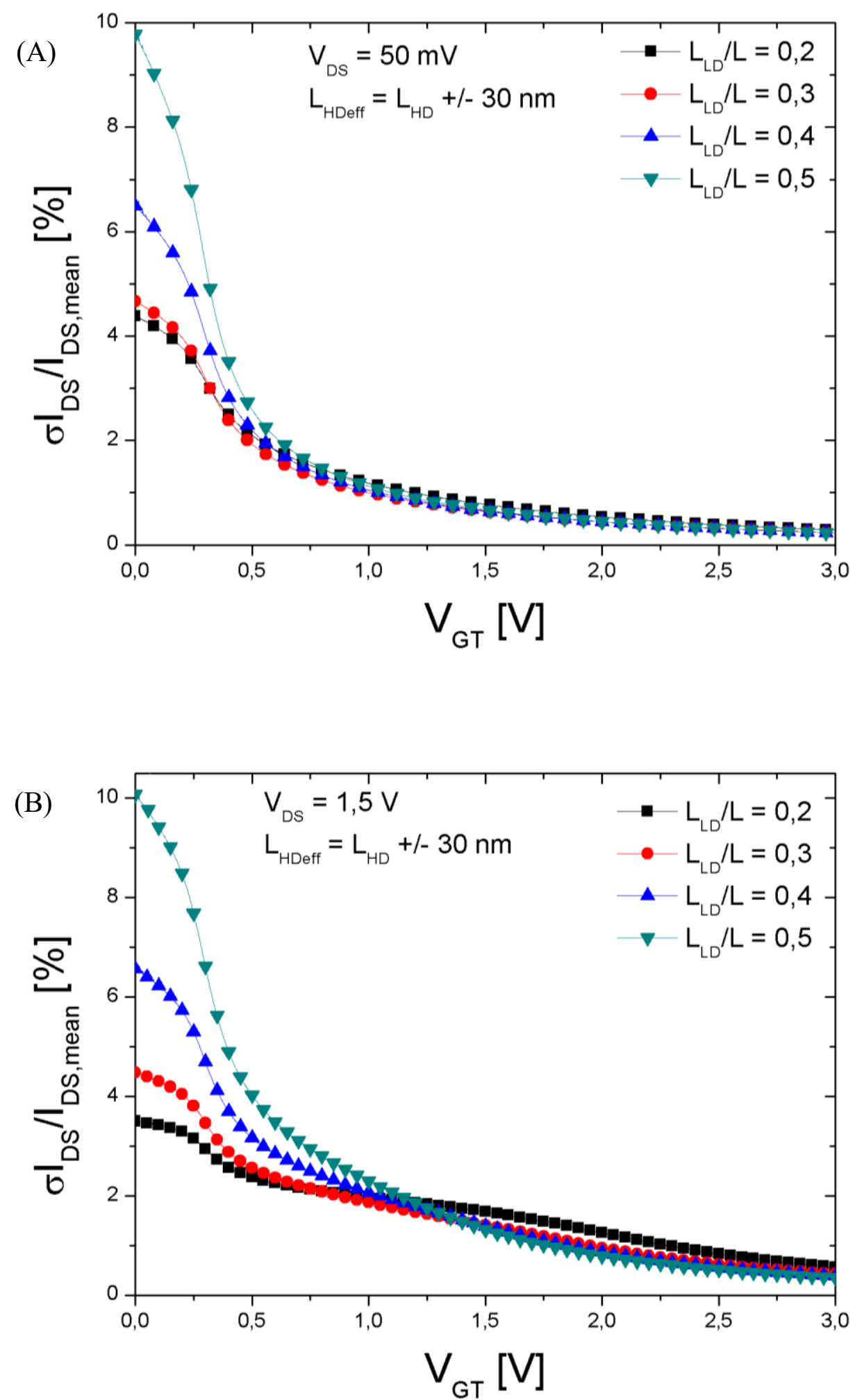


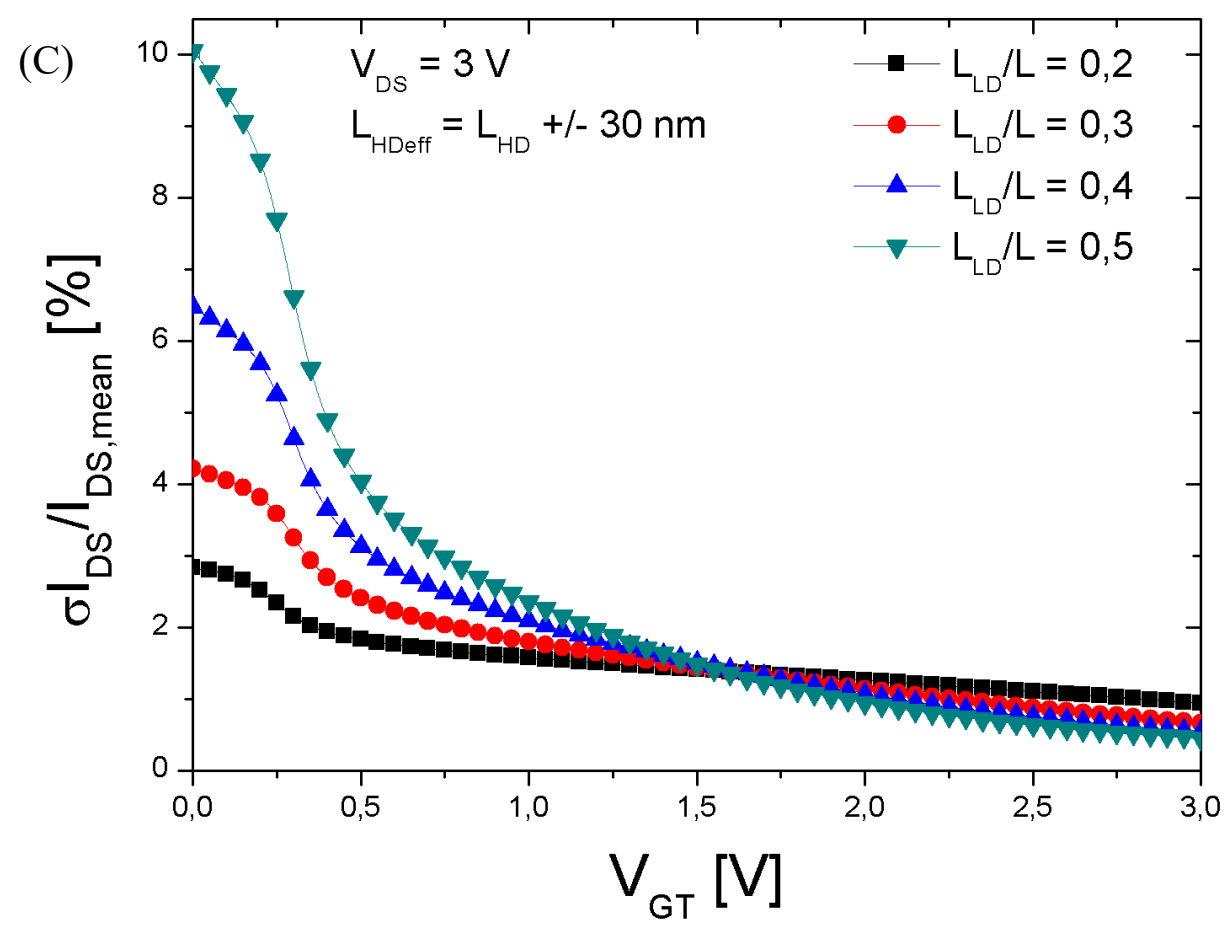

Fonte: Alves, 2017.

Pode-se observar que o descasamento da corrente de dreno é maior quando os dispositivos operam em inversão fraca. À medida que a sobretensão de condução aplicada aumenta, o dispositivo passa a operar em inversão forte e o desvio relativo diminui. Como exemplo, pode-se citar a razão $\mathrm{L}_{\mathrm{LD}} / \mathrm{L}=0,4 \mathrm{com} \mathrm{V}_{\mathrm{DS}}=1,5 \mathrm{~V}$ no qual em inversão fraca $\left(\mathrm{V}_{\mathrm{GT}}=\right.$ $0,25 \mathrm{~V})$ o desvio relativo é de aproximadamente 5,3\% e em inversão forte $\left(\mathrm{V}_{\mathrm{GT}}=2,8 \mathrm{~V}\right)$ é de aproximadamente $0,44 \%$. Conforme aumentamos a polarização de dreno, o desvio relativo da corrente em inversão forte aumenta, com $\mathrm{V}_{\mathrm{DS}}=3 \mathrm{~V}$, para os mesmos pontos, os desvios relativos são de aproximadamente $5,25 \%$ com $V_{\text {GT }}$ de $0,25 \mathrm{~V}$ e aproximadamente $0,61 \%$ com $\mathrm{V}_{\mathrm{GT}}$ de 2,8 V. Em inversão fraca o desvio relativo da corrente é maior que a variação imposta no comprimento efetivo de canal, seguindo o exemplo anterior, para a razão $L_{L D} / L=0,4$ o desvio relativo da corrente passa a ser menor do que a variação imposta no comprimento efetivo de canal a partir de $\mathrm{V}_{\mathrm{GT}}=0,74 \mathrm{~V}$.

Posteriormente foi traçada a curva do desvio relativo da transcondutância em função da sobretensão de condução com polarização de dreno de $50 \mathrm{mV}, 1.5 \mathrm{~V}$ e $3 \mathrm{~V}$, os resultados são apresentados conforme Figura 37. 
Figura 37 - Desvio relativo da transcondutância em função da sobretensão de condução de $\mathrm{V}_{\mathrm{DS}}=50 \mathrm{mV}(\mathrm{A}), 1,5 \mathrm{~V}(\mathrm{~B})$ e $3 \mathrm{~V}(\mathrm{C})$, com $\mathrm{L}_{\mathrm{HD}}$ variando $\pm 30 \mathrm{~nm}$.

(A)

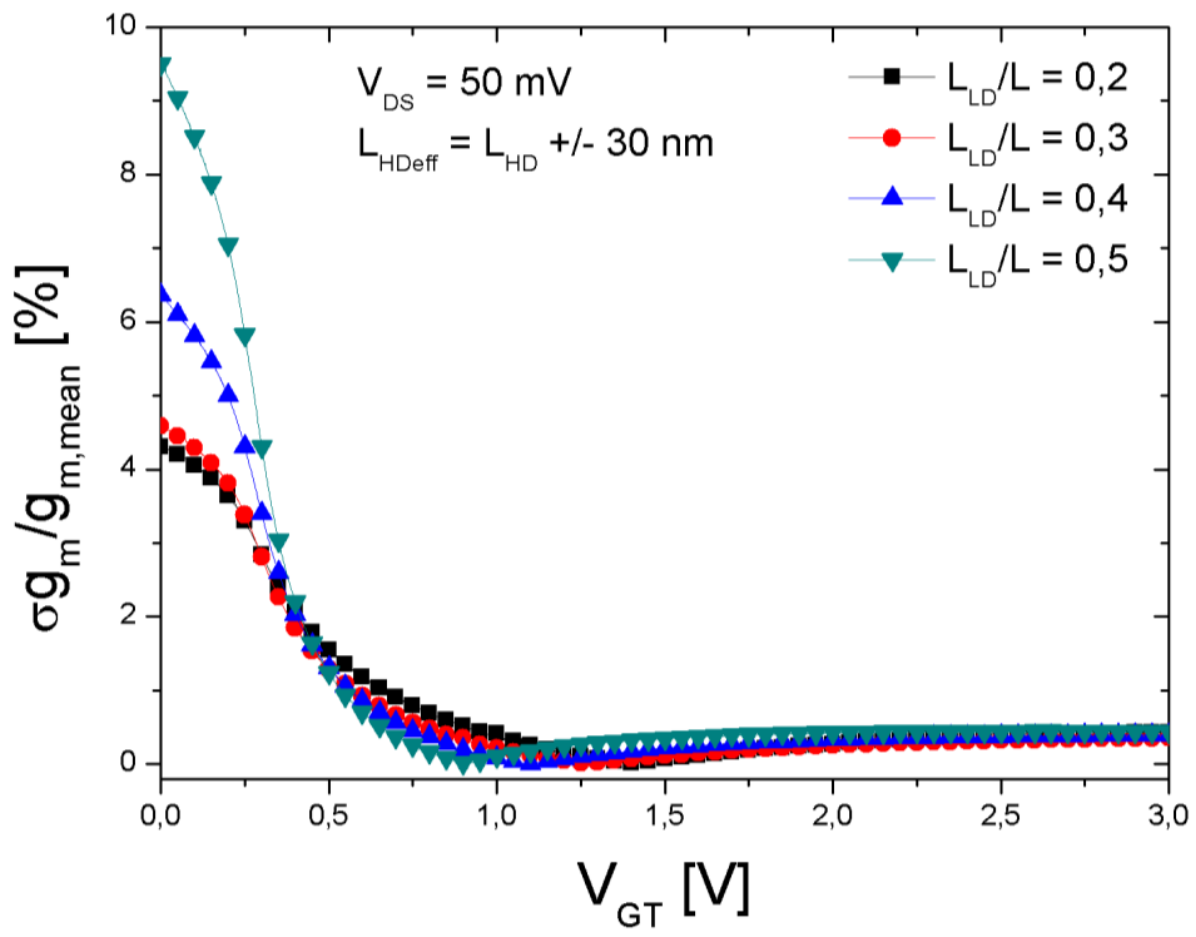

(B)

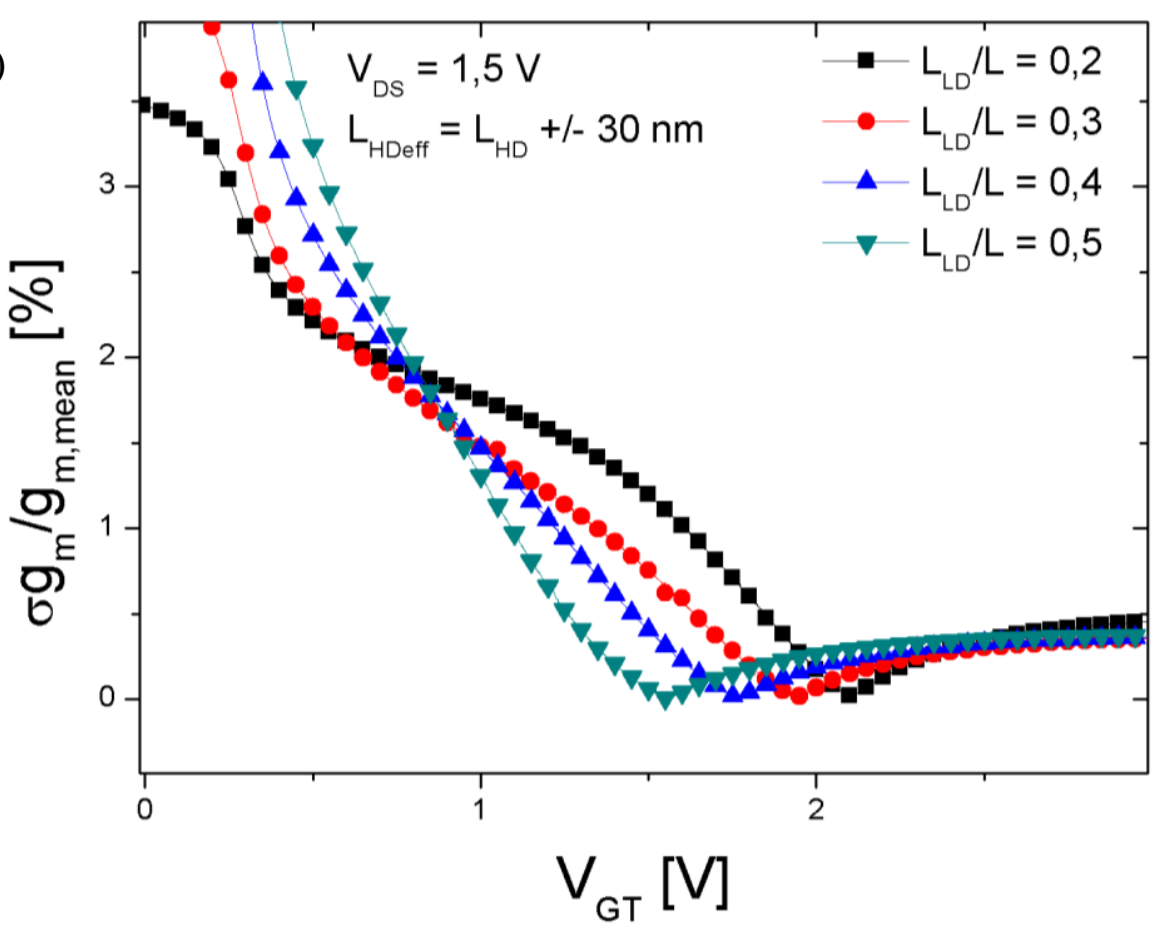


(C)

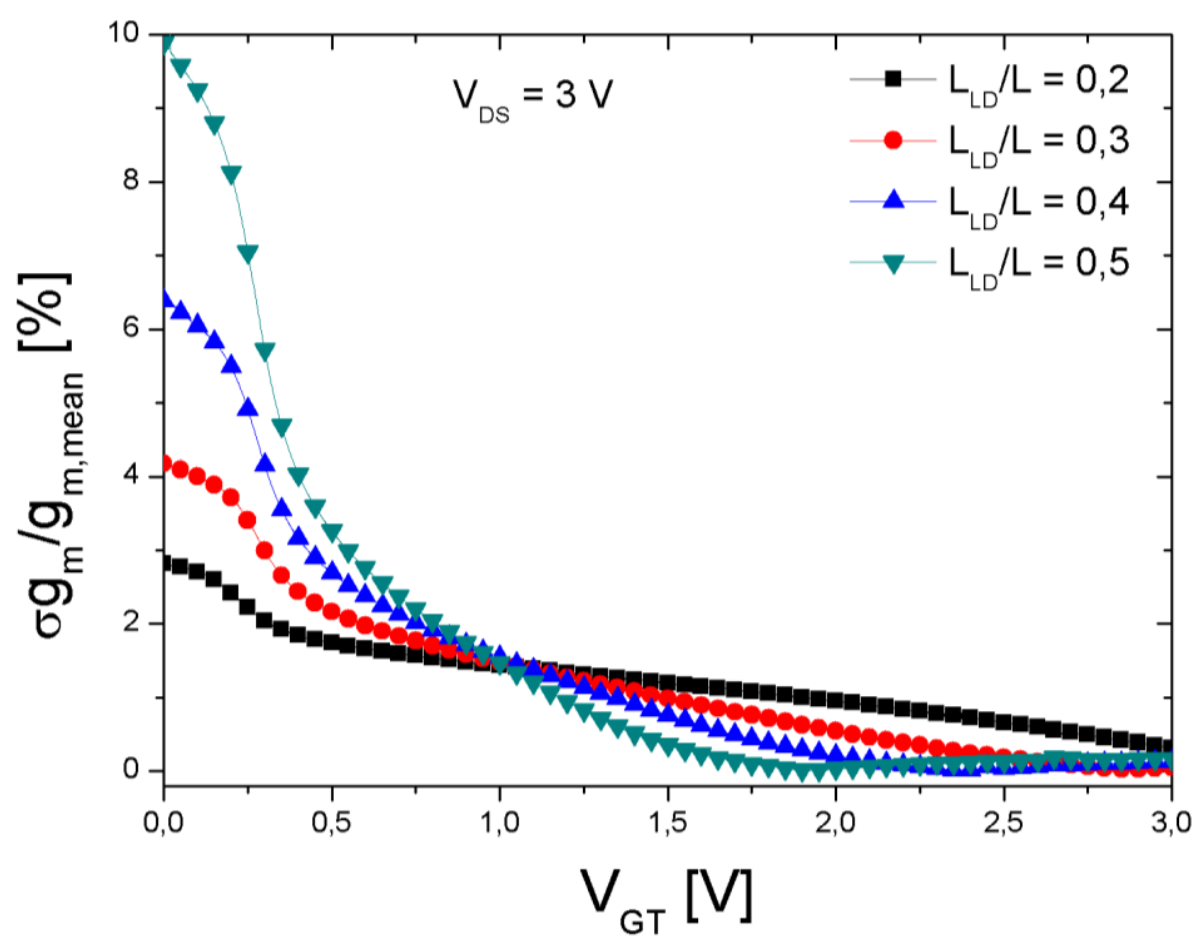

Fonte: Alves, 2017.

É possível notar que conforme aumentamos a sobretensão de condução o desvio relativo da transcondutância tende a um valor inferior a $1 \%$ para todos as tensões de dreno aplicadas. Conforme aumentamos a polarização de dreno o desvio relativo aumenta, como exemplo a razão $\mathrm{L}_{\mathrm{LD}} / \mathrm{L}=0,3 \mathrm{em} \mathrm{V}_{\mathrm{GT}}=2,5 \mathrm{~V}$ tem aproximadamente $0,3 \%$ com $\mathrm{V}_{\mathrm{DS}}$ de $1,5 \mathrm{~V}$ e aproximadamente $0,88 \%$ com $\mathrm{V}_{\mathrm{DS}}$ de $3 \mathrm{~V}$.

A partir dos resultados calculados foram extraídos os valores médios e desvios relativos da transcondutância para $\mathrm{V}_{\mathrm{GT}}=200 \mathrm{mV}$ e $\mathrm{V}_{\mathrm{DS}}=1,5 \mathrm{~V}$, conforme apresentado na Tabela 13. Pode-se observar que a variação imposta no comprimento de canal efetivo é transferida diretamente ao desvio relativo da transcondutância, como exemplo a razão $\mathrm{L}_{\mathrm{LD}} / \mathrm{L}=$ 0,2 foi variado em $1,875 \%$ e apresentou um desvio relativo da transcondutância de $1,93 \%$. 
Tabela 13 - Valor médio e desvio relativo da transcondutância para GC SOI MOSFETs, extraído para $\mathrm{V}_{\mathrm{GT}}=200 \mathrm{mV}$ e $\mathrm{V}_{\mathrm{DS}}=1,5 \mathrm{~V}$.

\begin{tabular}{ccc}
\hline $\mathbf{L} \mathbf{L D} / \mathbf{L}$ & $\mathrm{g}_{\mathrm{m}, \text { mean }}[\mu \mathrm{S}]$ & $\sigma \mathrm{g}_{\mathrm{m}} / \mathrm{g}_{\mathrm{m}, \text { mean }}[\%]$ \\
\hline $\mathbf{0 , 2}$ & 7,5 & 1,96 \\
\hline $\mathbf{0 , 3}$ & 8,71 & 2,02 \\
\hline $\mathbf{0 , 4}$ & 10,28 & 2,26 \\
\hline $\mathbf{0 , 5}$ & 12,60 & 3,21 \\
\hline
\end{tabular}

Fonte: Alves, $20 \overline{17 .}$

Em posse dos valores da tensão de limiar para cada dispositivo, foram simuladas as curvas IDS em função de $V_{D S}$ com sobretensão de condução de $0 \mathrm{mV}, 200 \mathrm{mV}, 400 \mathrm{mV}, 600$ $\mathrm{mV}$ e $800 \mathrm{mV}$, como exemplo a curva $\mathrm{I}_{\mathrm{DS}}$ em função de $\mathrm{V}_{\mathrm{DS}}$ com sobretensão de condução de $400 \mathrm{mV}$ conforme pode ser visto na Figura 38.

Figura 38 - Curva IDs em função de $V_{\mathrm{DS}}$ com sobretensão de condução $\mathrm{V}_{\mathrm{GT}}=400 \mathrm{mV}$ com $\mathrm{L}_{\mathrm{HD}}$ variando $\pm 30 \mathrm{~nm}$.

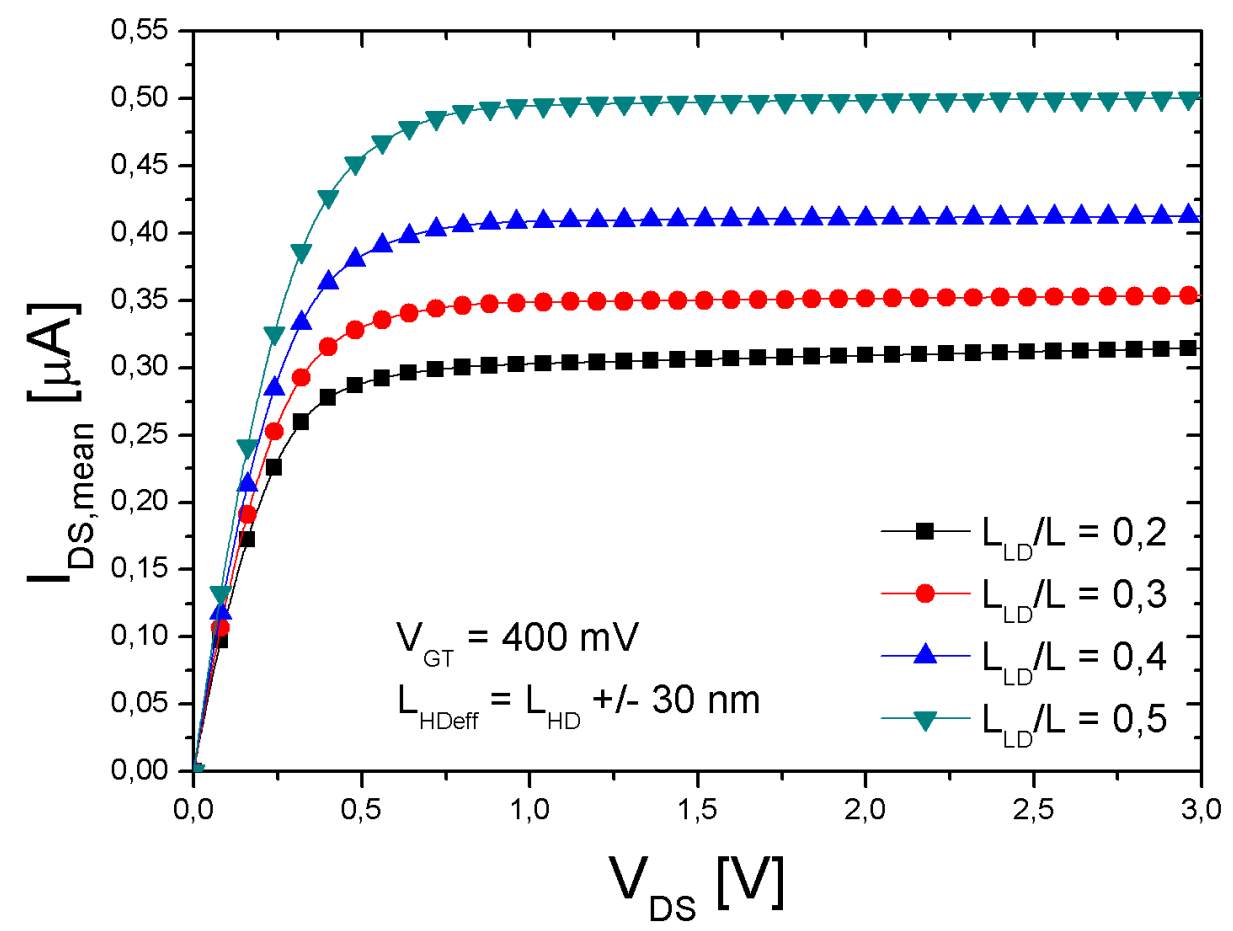

Fonte: Alves, 2017.

Pode ser observado que conforme aumenta a razão $\mathrm{L}_{\mathrm{LD}} / \mathrm{L}$, a corrente aumenta e ao atingir a saturação a corrente permanece praticamente constante. Conforme sobretensão de 
condução é aumentada, os valores das correntes aumentam. A partir destas curvas, foi extraído o desvio relativo da corrente de dreno em função da tensão de dreno, conforme apresentado na Figura 39.

Figura 39 - Desvio relativo da corrente de dreno em função de $\mathrm{V}_{\mathrm{DS}}$ com sobretensão de condução $\mathrm{V}_{\mathrm{GT}}=0 \mathrm{mV}$ (A), $200 \mathrm{mV}$ (B), $400 \mathrm{mV}$ (C), $600 \mathrm{mV}$ (D) e $800 \mathrm{mV}$ (E), com L $\mathrm{L}_{\mathrm{HD}}$ variando $\pm 30 \mathrm{~nm}$.

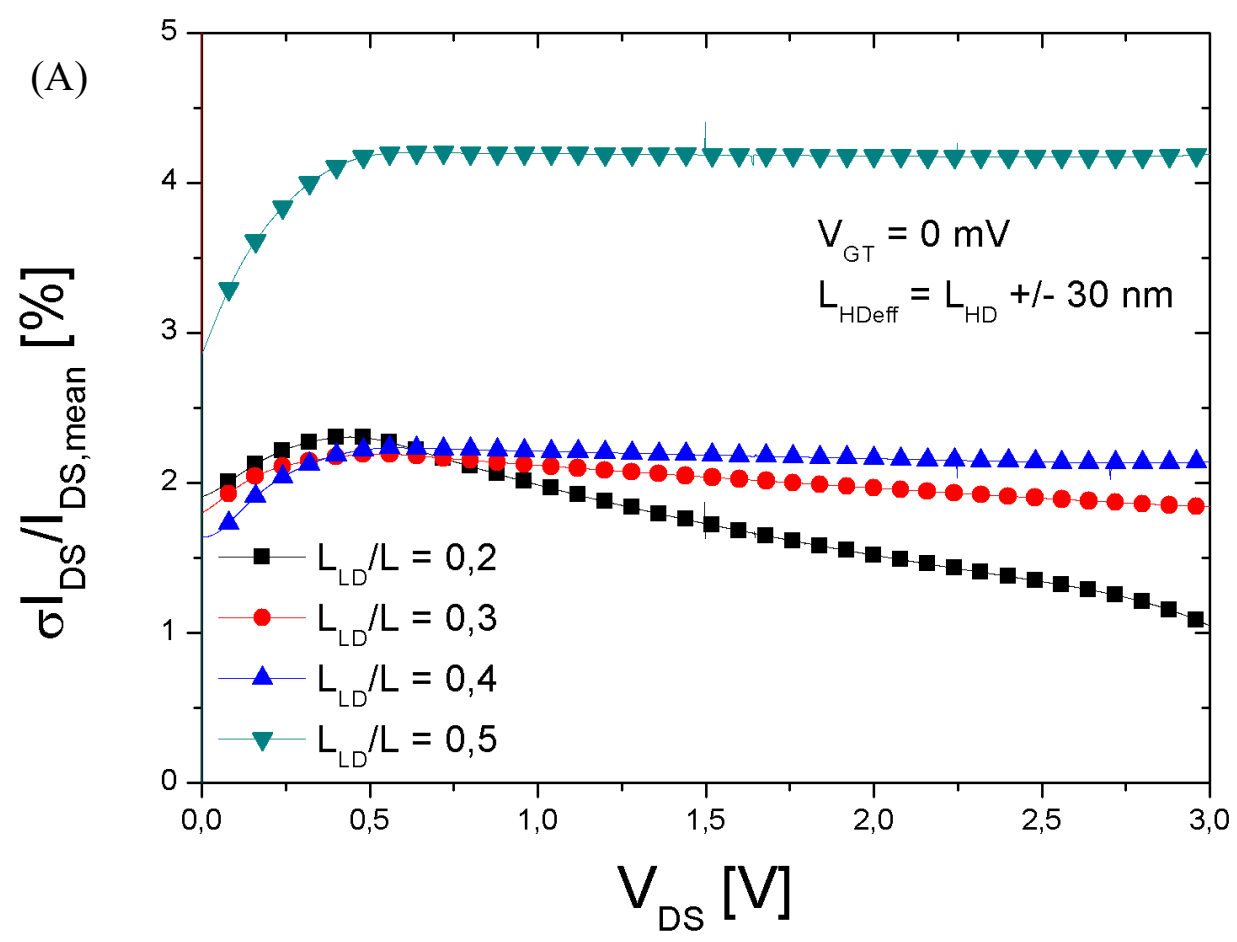



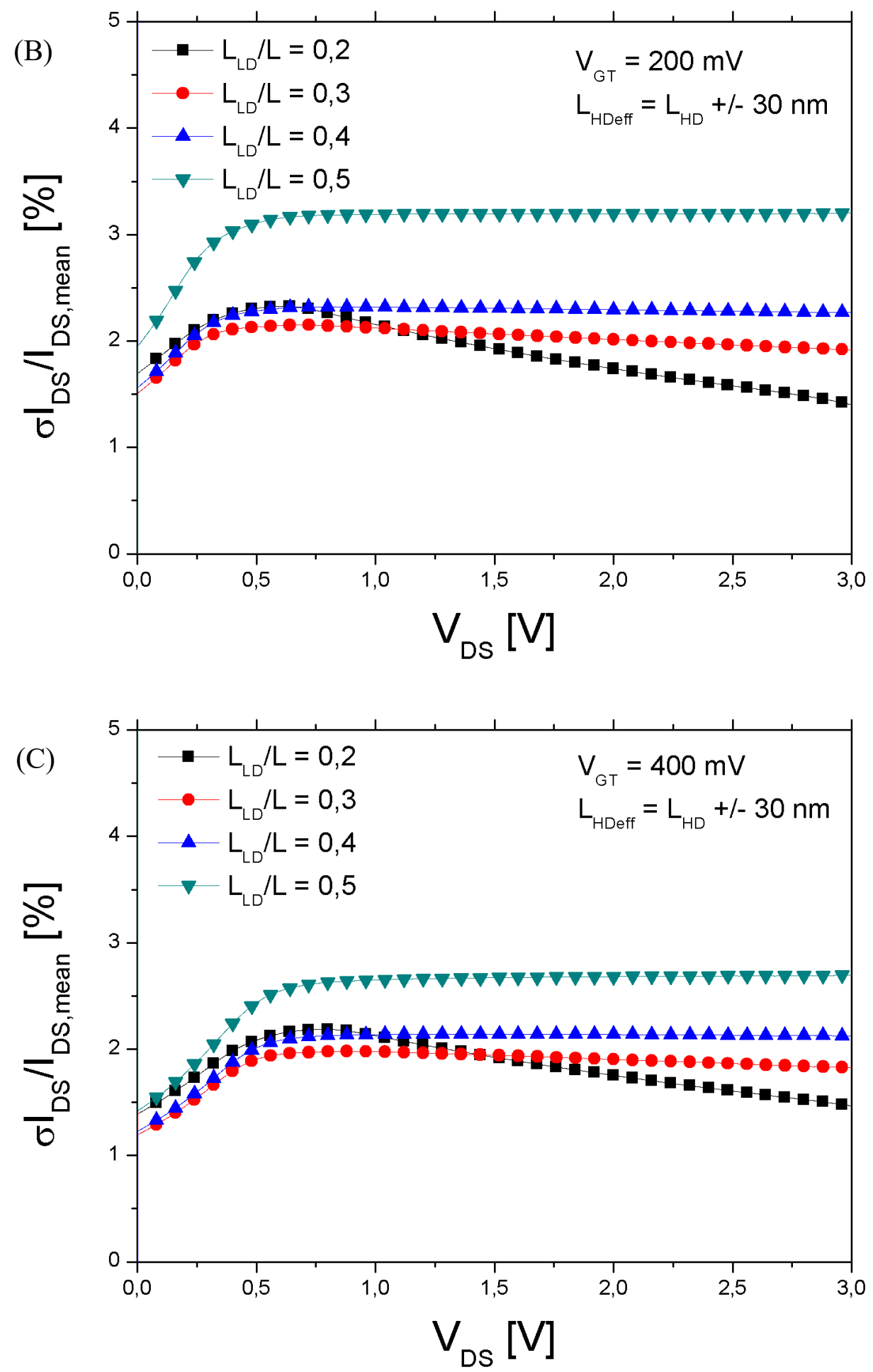

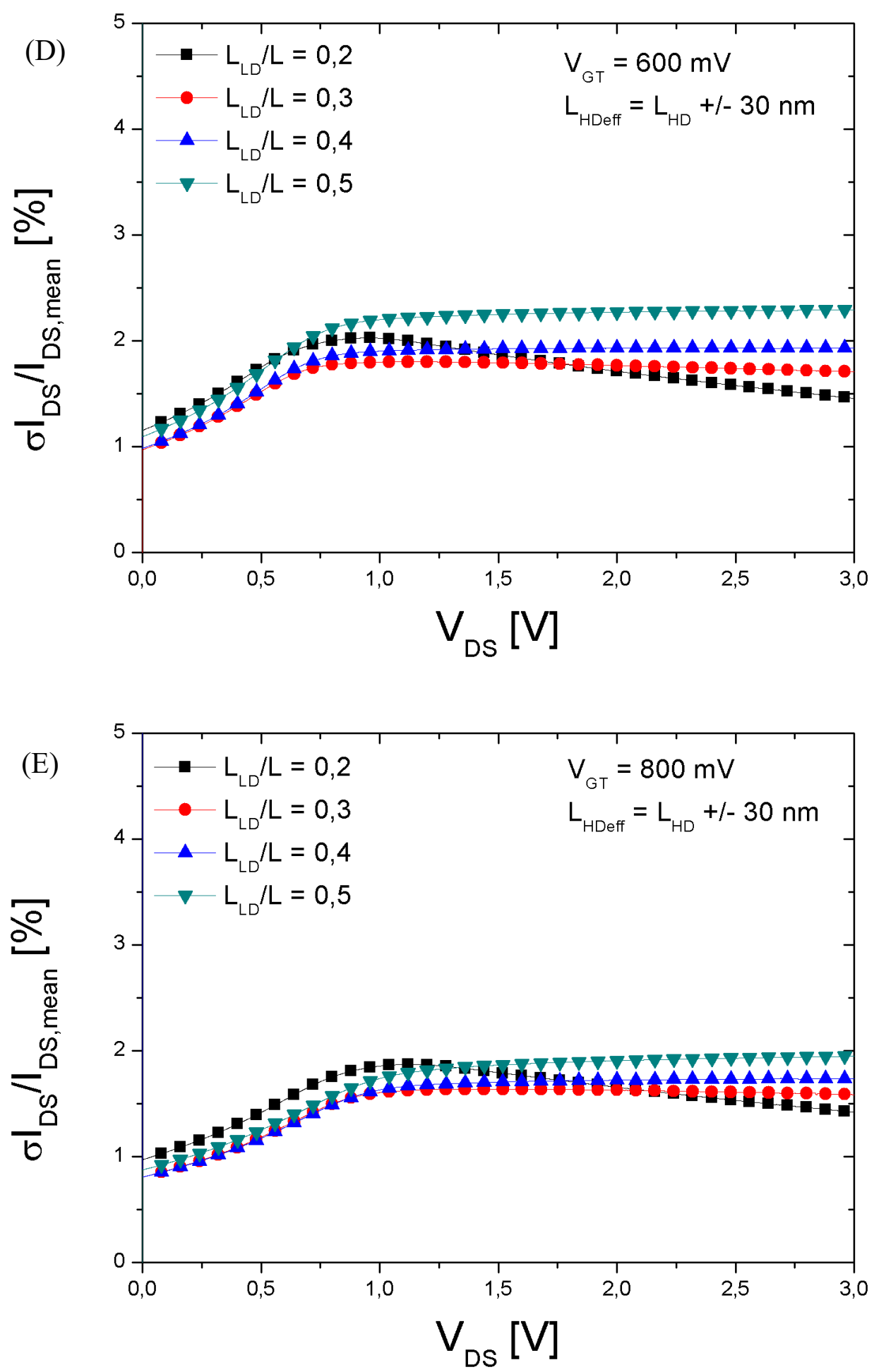

Fonte: Alves, 2017.

Pode-se observar que conforme se aumenta a sobretensão de condução o desvio relativo tende a diminuir, como exemplo a razão $\mathrm{L}_{\mathrm{LD}} / \mathrm{L}=0,3 \mathrm{em} \mathrm{V}_{\mathrm{DS}}=2 \mathrm{~V}$ tem-se um desvio relativo de aproximadamente $2 \%$ com $\mathrm{V}_{\mathrm{GT}}=200 \mathrm{mV}$ e aproximadamente $1,8 \%$ com $\mathrm{V}_{\mathrm{GT}}=$ $600 \mathrm{mV}$. A variação de $\pm 30 \mathrm{~nm}$ na razão $\mathrm{L}_{\mathrm{LD}} / \mathrm{L}$ de 0,3 resulta em um desvio relativo de 
aproximadamente 2,143\%, quando aplicada uma sobretensão de condução mais baixa (no caso do exemplo - $2 \%$ ) essa variação é transferida diretamente ao desvio relativo da corrente de dreno, já para valores mais altos da sobretensão de condução (no caso do exemplo - 1,8\%), pode-se observar uma leve atenuação no desvio relativo da corrente.

A partir das curvas de corrente, foram extraídos os valores de condutância de dreno para cada dispositivo e calculado o valor médio e seu desvio relativo, conforme pode-se observar na Tabela 14.

Tabela 14 - Valor médio e desvio relativo da condutância de dreno para GC SOI MOSFETs, extraídos para $\mathrm{V}_{\mathrm{GT}}=200 \mathrm{mV}$ e $\mathrm{V}_{\mathrm{DS}}=1,5 \mathrm{~V}$.

\begin{tabular}{|c|c|c|}
\hline $\mathbf{L}_{\mathbf{L D}} / \mathbf{L}$ & $\mathrm{g}_{\mathrm{D}, \text { mean }}[\mathrm{nS}]$ & $\sigma \mathrm{g}_{\mathrm{D}} / \mathrm{g}_{\mathrm{D}, \text { mean }}[\%]$ \\
\hline 0,2 & 21,04 & 15,65 \\
\hline 0,3 & 8,17 & 11,58 \\
\hline 0,4 & 4,09 & 5,62 \\
\hline 0,5 & 4,17 & 5,11 \\
\hline
\end{tabular}

Fonte: Alves, $20 \overline{17 .}$

Pode-se observar que o desvio relativo da condutância de dreno é superior as variações impostas no comprimento efetivo de canal, como exemplo a razão $\mathrm{L}_{\mathrm{LD}} / \mathrm{L}=0,3$ que sofreu uma variação de $2,143 \%$ no comprimento efetivo do canal e apresenta um desvio relativo da condutância de dreno de $11,58 \%$.

Foram calculados os valores de tensão Early, com sobretensão de condução de 200 $\mathrm{mV}$, conforme Figura 40. 
Figura 40 - Tensão Early em função da tensão de dreno com sobretensão de condução $\mathrm{V}_{\mathrm{GT}}=$ $200 \mathrm{mV}$ com $\mathrm{L}_{\mathrm{HD}}$ variando $\pm 30 \mathrm{~nm}$.

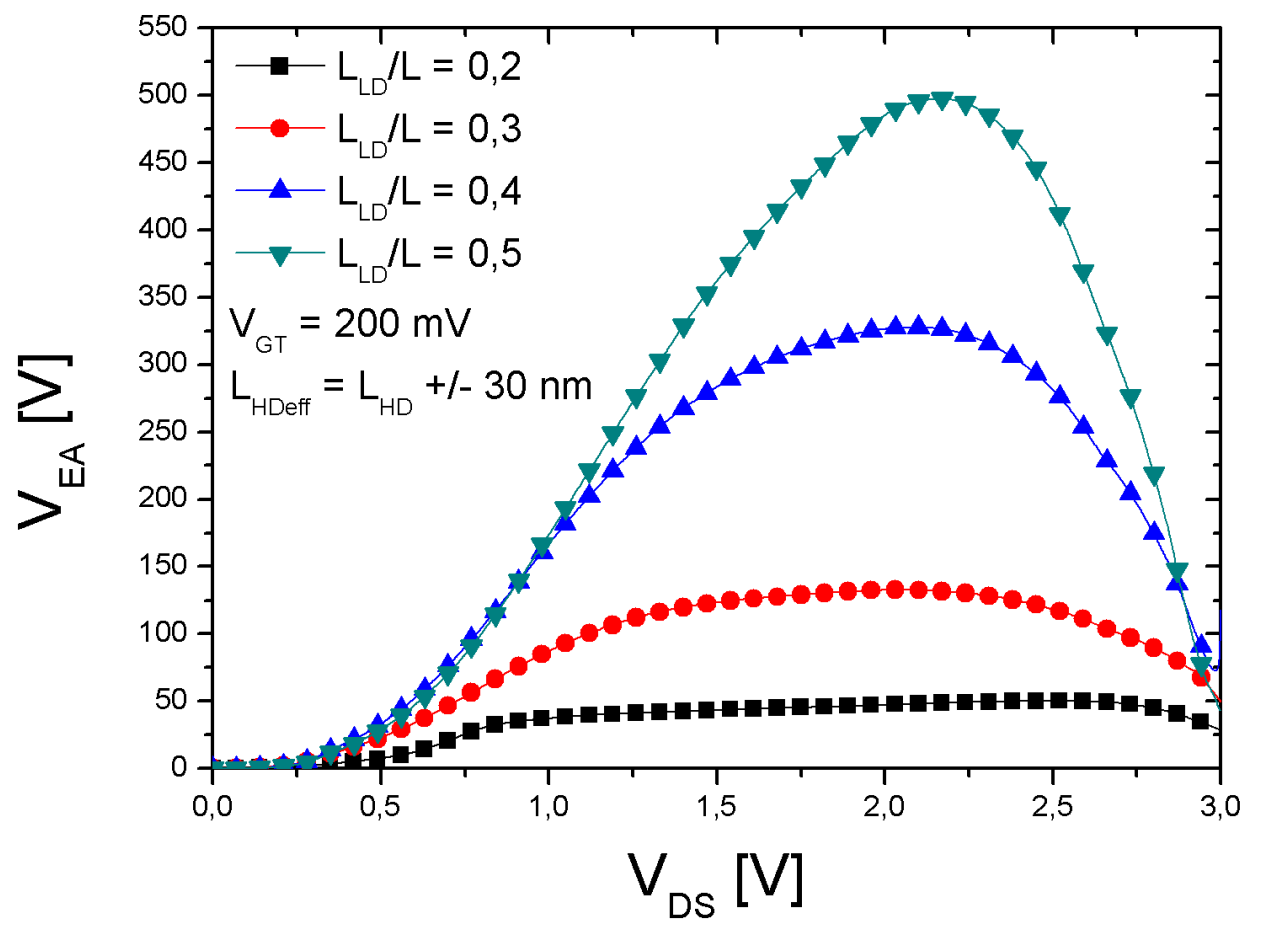

Fonte: Alves, 2017.

Para a tensão de dreno de 1,5 V foram extraídos os valores da tensão Early médias e os desvios relativos conforme apresentado na Tabela 15.

Tabela 15 - Tensão Early e desvio relativo obtidas a partir das simulações dos GC SOI MOSFETs com $\mathrm{V}_{\mathrm{GT}}=200 \mathrm{mV}$ e $\mathrm{V}_{\mathrm{DS}}=1,5 \mathrm{~V}$ com $\mathrm{L}_{\mathrm{HD}}$ variando $\pm 30 \mathrm{~nm}$.

\begin{tabular}{ccc}
\hline $\mathbf{L}_{\mathbf{L D}} / \mathbf{L}$ & $\mathrm{V}_{\mathrm{EA}, \text { mean }}[\mathrm{V}]$ & $\sigma \mathrm{V}_{\mathrm{EA}} / \mathrm{V}_{\mathrm{EA}, \text { mean }}[\%]$ \\
\hline $\mathbf{0 , 2}$ & 43,37 & 17,61 \\
\hline $\mathbf{0 , 3}$ & 127,69 & 14,09 \\
\hline $\mathbf{0 , 4}$ & 301,15 & 7,96 \\
\hline $\mathbf{0 , 5}$ & 367,14 & 2,05
\end{tabular}

Fonte: Alves, $20 \overline{17 .}$

Pode-se observar um aumento da tensão Early média conforme aumentamos a razão $\mathrm{L}_{\mathrm{LD}} / \mathrm{L}$ e o desvio relativo diminui. O desvio relativo da tensão Early mostrou-se maior do que 
a variação imposta no comprimento de canal efetivo. Como exemplo pode-se citar o transistor com $\mathrm{L}_{\mathrm{LD}} / \mathrm{L}$ de 0,4 que apresenta uma variação de $2,5 \%$ em relação a $\mathrm{L}_{\mathrm{HD}}$, enquanto o desvio relativo observado para a tensão Early foi de $7,12 \%$.

Foi calculado a ganho de tensão $\left(\mathrm{A}_{\mathrm{V}}=\mathrm{g}_{\mathrm{m}} / \mathrm{g}_{\mathrm{D}}\right)$ para $\mathrm{V}_{\mathrm{GT}}=200 \mathrm{mV}$ e $\mathrm{V}_{\mathrm{DS}}=1,5 \mathrm{~V}$, para cada dispositivo. Posteriormente, os ganhos de tensão médios e os desvios relativos foram obtidos estão presentes na Tabela 16.

Tabela 16 - Ganho de tensão e desvio relativo dos GC SOI MOSFETs com $\mathrm{V}_{\mathrm{GT}}=200 \mathrm{mV}$ e $\mathrm{V}_{\mathrm{DS}}=1,5 \mathrm{~V}$ com $\mathrm{L}_{\mathrm{HD}}$ variando $\pm 30 \mathrm{~nm}$.

\begin{tabular}{ccc}
\hline $\mathbf{L}_{\mathbf{L D}} / \mathbf{L}$ & $\mathrm{A}_{\mathrm{V} \text {,mean }}[\mathrm{V} / \mathrm{V}]$ & $\sigma \mathrm{A}_{\mathrm{V}} / \mathrm{A}_{\mathrm{V}, \text { mean }}[\%]$ \\
\hline $\mathbf{0 , 2}$ & 367,23 & 17,68 \\
\hline $\mathbf{0 , 3}$ & 1078,46 & 14,05 \\
\hline $\mathbf{0 , 4}$ & 2525,40 & 7,92 \\
\hline $\mathbf{0 , 5}$ & 3022,65 & 2,42
\end{tabular}

Fonte: Alves, $20 \overline{17 .}$

Pode-se observar que conforme aumentamos a razão $\mathrm{L}_{\mathrm{LD}} / \mathrm{L}$, o ganho de tensão aumenta e o desvio relativo diminui. Assim como observado no caso da tensão Early, o desvio relativo do ganho de tensão mostrou-se maior do que a variação imposta no comprimento efetivo de canal

De forma geral, o desvio relativo da tensão de limiar e da inclinação de sublimiar é menor que a variação imposta no comprimento efetivo do canal. O desvio relativo da transcondutância acaba sendo aproximadamente igual à variação imposta, e os desvio da condutância de saída, da tensão Early e do ganho de tensão são maiores que a variação imposta.

\subsection{VARIAÇÃO DA CONCENTRAÇÃO DE DOPANTES}

Após a análise da influência da variação do comprimento efetivo de canal sobre o descasamento das características elétricas dos transistores de canal gradual, foi realizada a análise da influência de possíveis desvios da concentração de dopantes da região fortemente dopada do canal. 
Neste grupo de dispositivos simulados, as relações $\mathrm{L}_{\mathrm{LD}} / \mathrm{L}$ foram mantidas e foi imposta uma variação de $\pm 10 \%$ na concentração de dopantes da região fortemente dopada do canal, fazendo com que a mesma varie entre $4,5 \cdot 10^{16}$ à $5,5 \cdot 10^{16} \mathrm{~cm}^{-3}$.

Primeiramente foram extraídas as curvas de corrente de dreno em função da tensão de porta com passo de $1 \mathrm{mV}$, conforme mostra a Figura 41 .

Figura 41 - Curva $\mathrm{I}_{\mathrm{DS} \text {,mean }} \mathrm{em}$ função de $\mathrm{V}_{\mathrm{GT}}$ para os GC SOI MOSFETs com concentração de $5.10^{16} \mathrm{~cm}^{-3}$ variando $\pm 10 \%, \mathrm{~V}_{\mathrm{DS}}=50 \mathrm{mV}(\mathrm{A}), 1,5 \mathrm{~V}(\mathrm{~B})$ e $3 \mathrm{~V}(\mathrm{C})$ e $\mathrm{L}_{\mathrm{LD}} / \mathrm{L}$ de $0.2,0.3,0.4$, 0.5 .

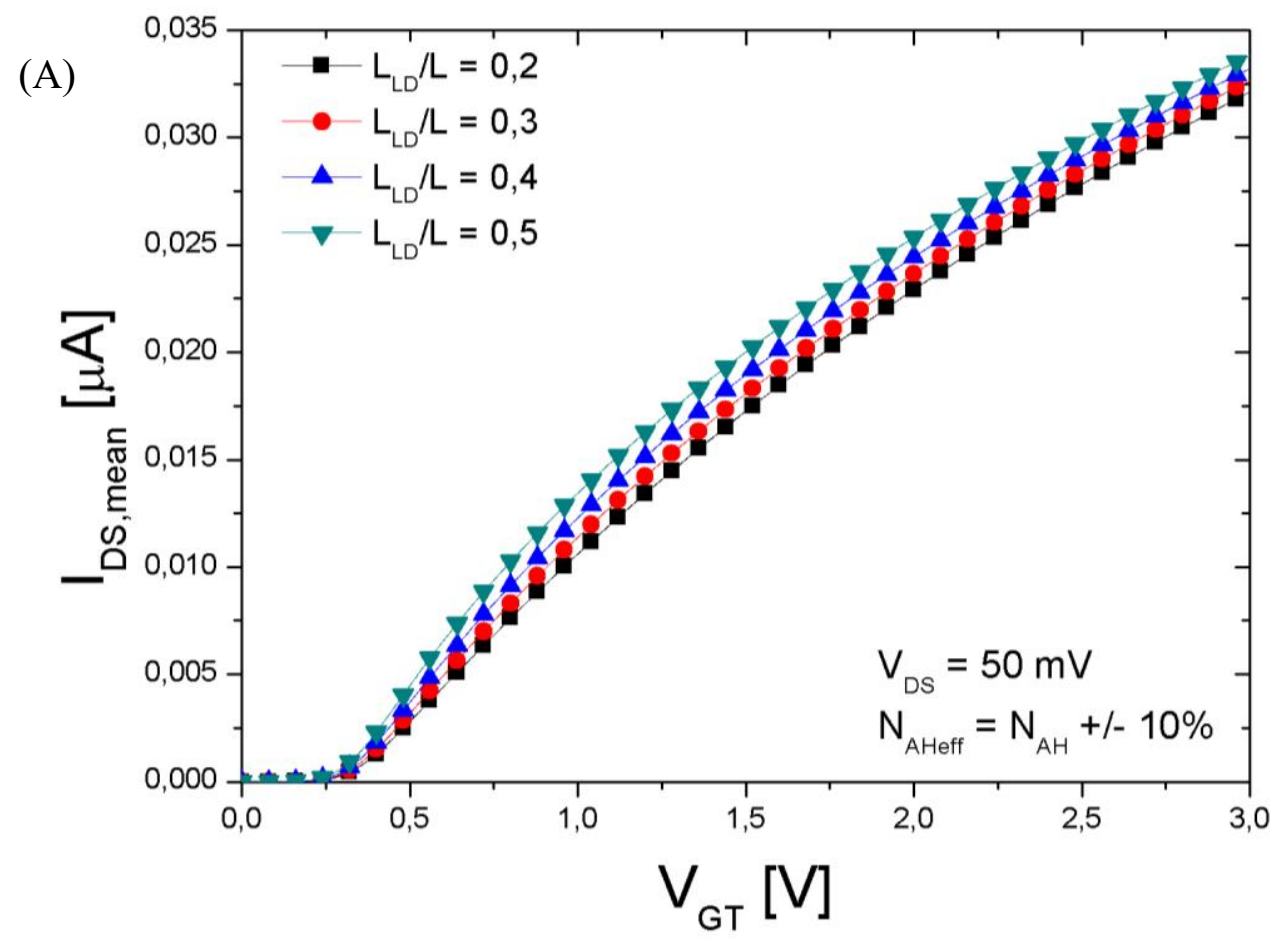


(B)

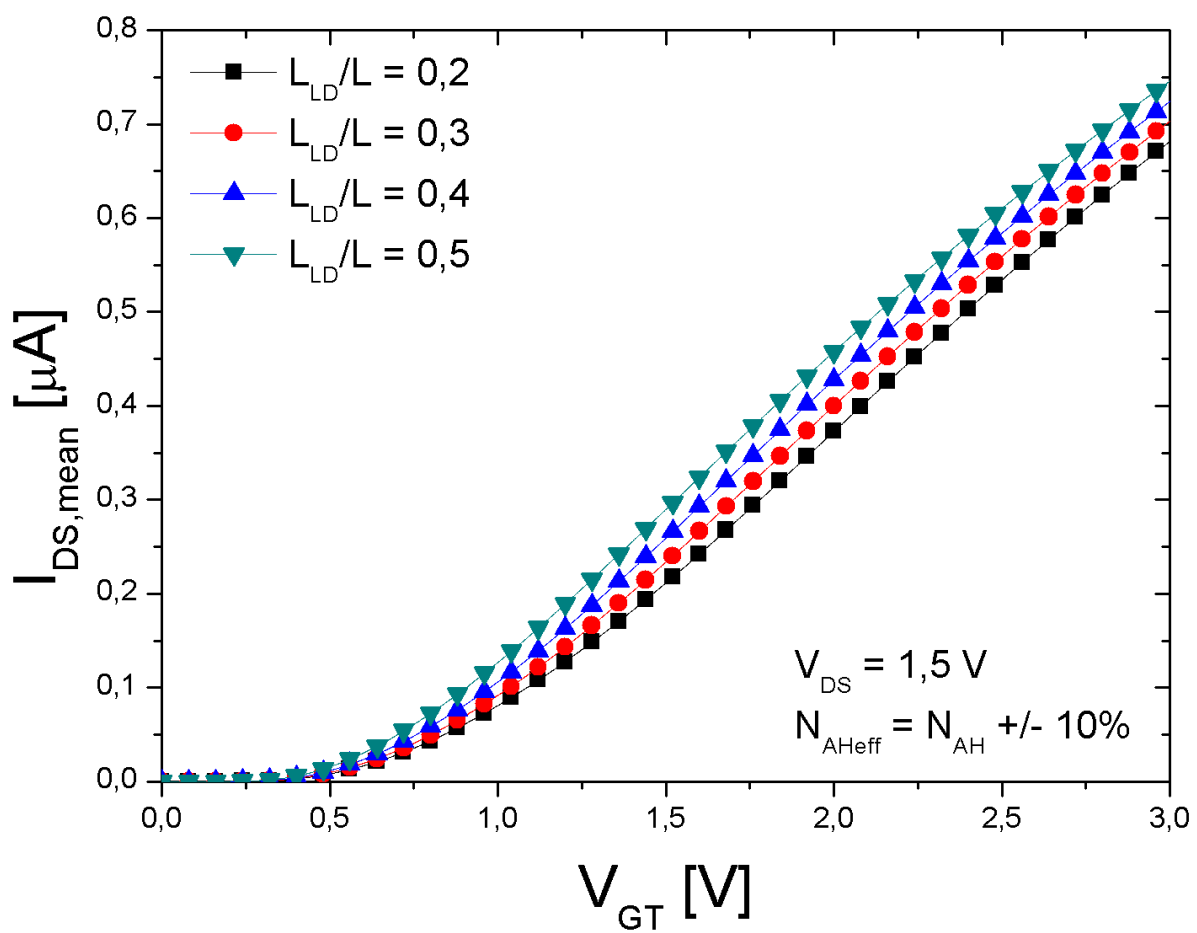

(C)

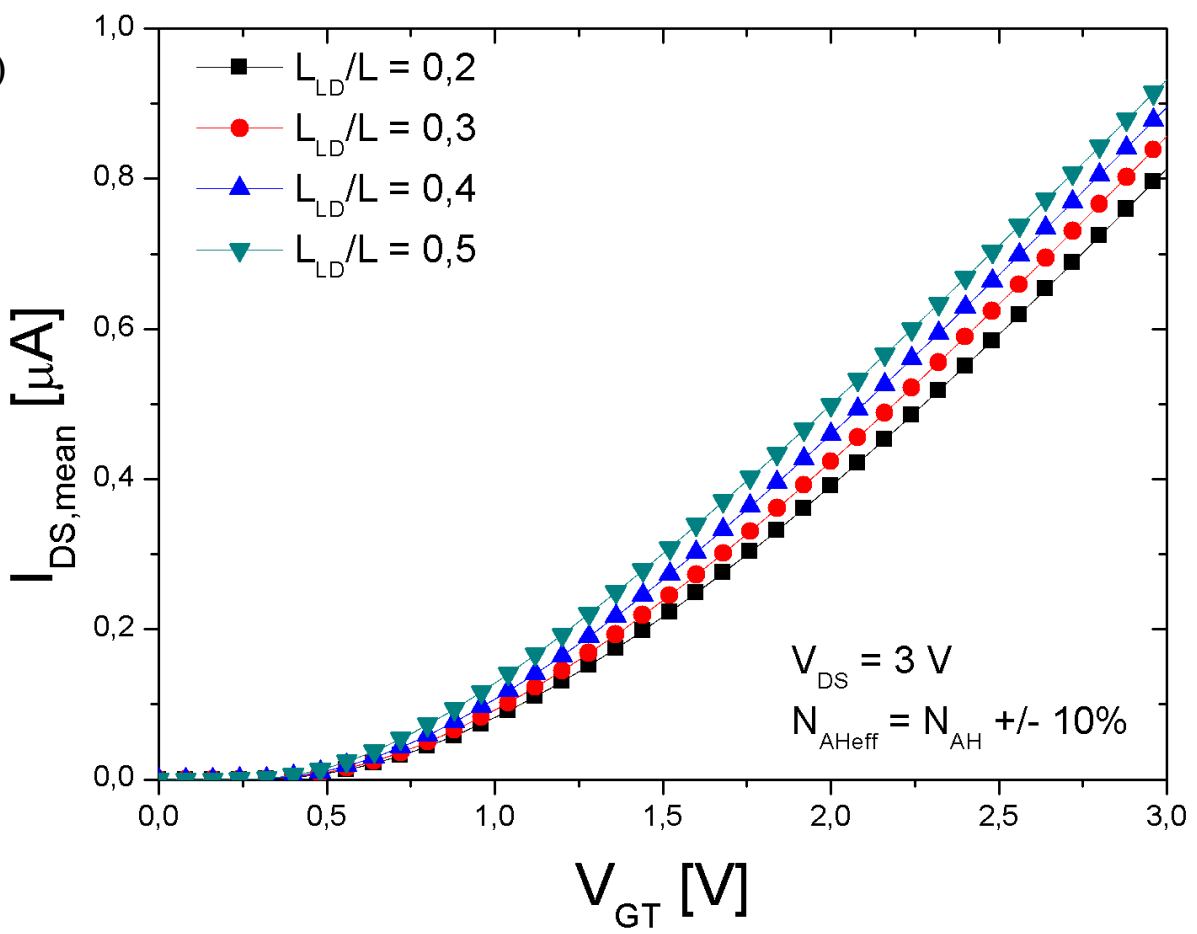

Fonte: Alves, 2017.

Foram extraídos os valores da tensão de limiar médio e desvio relativo, conforme apresentado na Tabela 17. 
Tabela 17 - Valor médio e desvio relativo da tensão de limiar de GC SOI MOSFETs com concentração de $5.10^{16} \mathrm{~cm}^{-3}$ variando $\pm 10 \%, \mathrm{~V}_{\mathrm{DS}}=50 \mathrm{mV}$ e $\mathrm{L}_{\mathrm{LD}} / \mathrm{L}$ de $0.2,0.3,0.4,0.5$.

\begin{tabular}{ccc}
\hline $\mathbf{L} \mathbf{L D} / \mathbf{L}$ & $\mathrm{V}_{\text {thf,mean }}[\mathrm{mV}]$ & $\sigma \mathrm{V}_{\text {thf }} / \mathrm{V}_{\text {thf,mean }}[\%]$ \\
\hline $\mathbf{0 , 2}$ & 318,67 & 18,35 \\
\hline $\mathbf{0 , 3}$ & 315,67 & 18,53 \\
\hline $\mathbf{0 , 4}$ & 311,00 & 18,97 \\
\hline $\mathbf{0 , 5}$ & 303,67 & 19,26
\end{tabular}

Fonte: Alves, $20 \overline{17 .}$

Pode-se observar que os valores da tensão de limiar médio estão semelhantes aos valores encontrados quando variado o comprimento efetivo do canal, porém resultou em um maior desvio relativo. Como exemplo, pode-se citar a razão $\mathrm{L}_{\mathrm{LD}} / \mathrm{L}=0,3$ que apresentou $\mathrm{V}_{\text {thf,mean }}=316 \mathrm{mV}$ e $\sigma \mathrm{V}_{\text {thf }} / \mathrm{V}_{\text {thf,mean }}=0,182 \%$, enquanto com a variação de $10 \%$ na concentração da região fortemente dopada, resultou em 18,53\% de desvio em $\mathrm{V}_{\text {thf. Assim, }}$ pode-se concluir que as variações de tensão de limiar obtidas experimentalmente, devem-se principalmente às flutuações na concentração de dopantes na região fortemente dopada do canal.

A Tabela 18 apresenta a inclinação de sublimiar média e seu desvio relativo. Pode-se observar que os valores médios obtidos para a inclinação de sublimiar médio são bastante semelhantes aos valores encontrados quando variado o comprimento efetivo do canal, porém há um aumento do desvio relativo. Diferentemente do resultado obtido quando variado o comprimento efetivo do canal, a variação da concentração de dopantes gerou uma influência no desvio da inclinação de sublimiar.

Tabela 18 - Valor médio e desvio relativo da inclinação de sublimiar de GC SOI MOSFETs com concentração de $5.10^{16} \mathrm{~cm}^{-3}$ variando $\pm 10 \%, \mathrm{~V}_{\mathrm{DS}}=50 \mathrm{mV}$ e $\mathrm{L}_{\mathrm{LD}} / \mathrm{L}$ de $0.2,0.3,0.4,0.5$.

\begin{tabular}{ccc}
\hline $\mathbf{L}_{\mathbf{L D}} / \mathbf{L}$ & $\mathrm{S}_{\text {mean }}[\mathrm{mV} / \mathrm{dec}]$ & $\sigma \mathrm{S} / \mathrm{S}_{\text {mean }}[\%]$ \\
\hline $\mathbf{0 , 2}$ & 65,7 & 0,30 \\
\hline $\mathbf{0 , 3}$ & 66,1 & 0,31 \\
\hline $\mathbf{0 , 4}$ & 66,9 & 0,48 \\
\hline $\mathbf{0 , 5}$ & 68,3 & 0,44 \\
\hline
\end{tabular}

Fonte: Alves, $20 \overline{17 .}$ 
Posteriormente, foi traçada a curva do desvio relativo da corrente de dreno em função da sobretensão de condução com polarização de dreno de $50 \mathrm{mV}, 1.5 \mathrm{~V}$ e $3 \mathrm{~V}$, os resultados são apresentados conforme apresentado na Figura 42.

Figura 42 - Desvio relativo da corrente de dreno em função da sobretensão de condução de $\mathrm{V}_{\mathrm{DS}}=50 \mathrm{mV}(\mathrm{A}), 1,5 \mathrm{~V}(\mathrm{~B})$ e $3 \mathrm{~V}(\mathrm{C})$, com concentração de $5.10^{16} \mathrm{~cm}^{-3}$ variando $\pm 10 \%$.
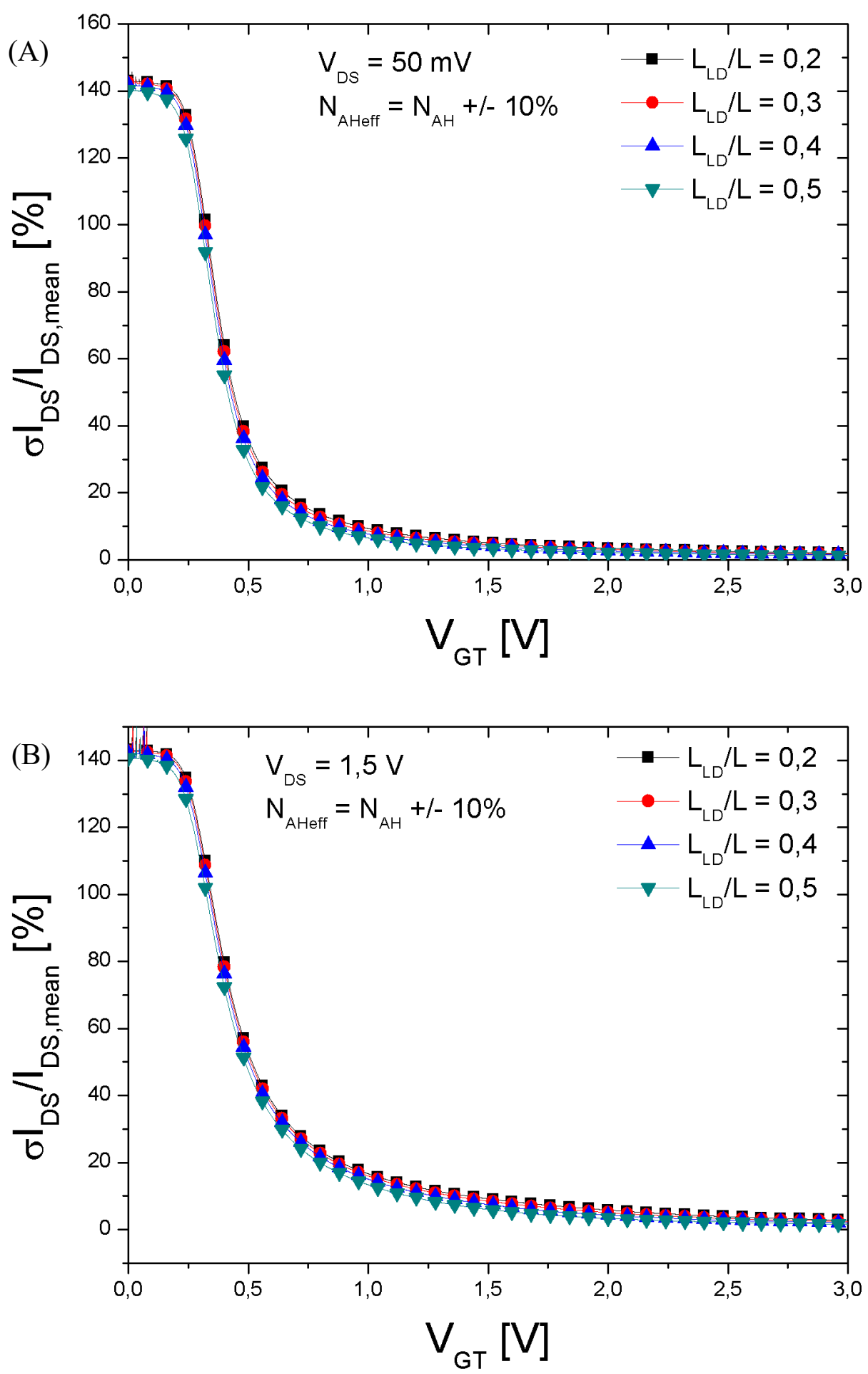


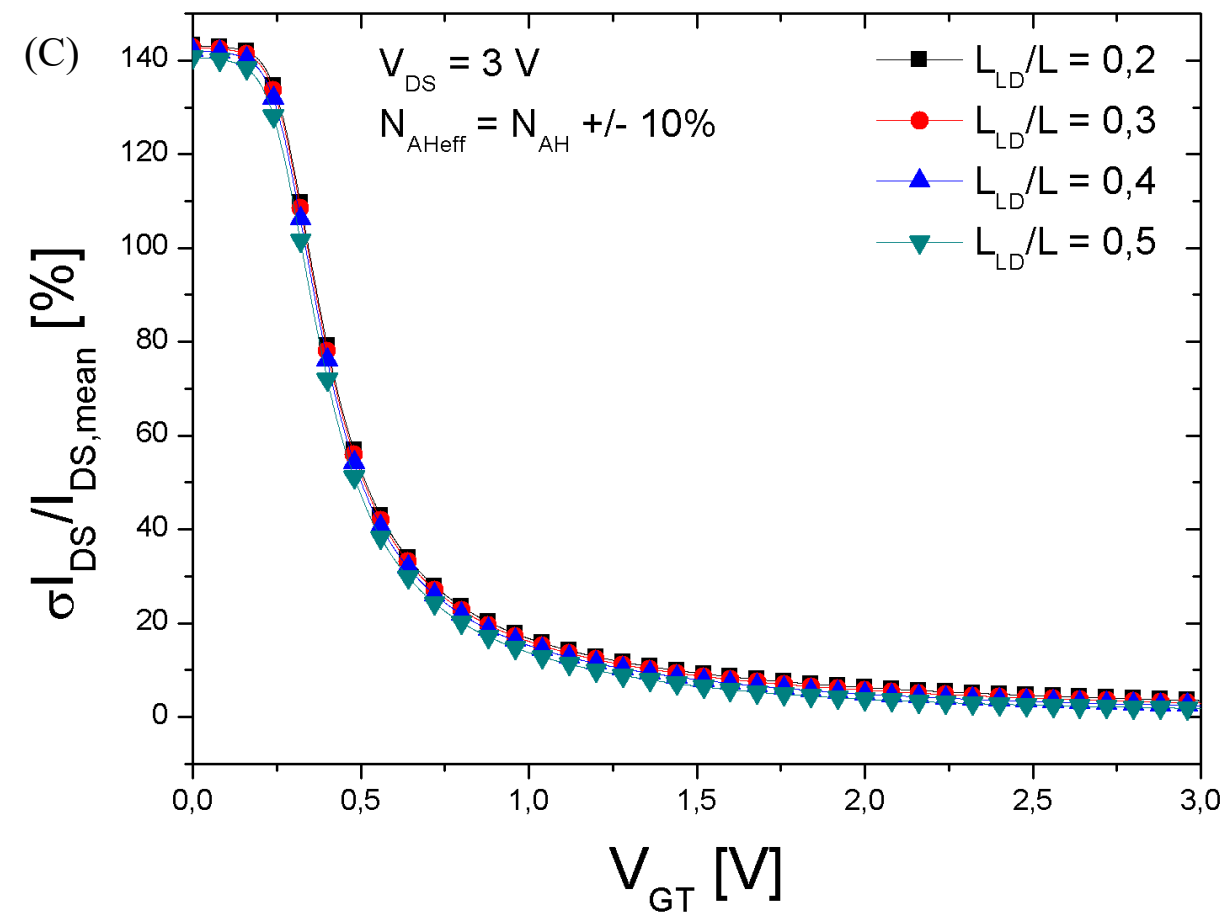

Fonte: Alves, 2017.

Pode ser observado um aumento do desvio relativo quando variada a concentração de dopantes em relação aos desvios relativos obtidos com a variação do comprimento efetivo de canal. Como exemplo usado anteriormente, com a razão $\mathrm{L}_{\mathrm{LD}} / \mathrm{L}=0,4$ e $\mathrm{V}_{\mathrm{DS}}=1,5 \mathrm{~V}$ obteve-se os valores de $5,3 \%$ e $0,44 \%$ para $\mathrm{V}_{\mathrm{GT}}$ de $0,25 \mathrm{~V}$ e $2,8 \mathrm{~V}$ respectivamente, para a variação da concentração de dopantes tem-se os desvio relativos de $129 \%$ e 2,23\% nos mesmos pontos. Conforme aumentamos a polarização de dreno o desvio relativo aumenta, como exemplo a razão $\mathrm{L}_{\mathrm{LD}} / \mathrm{L}=0,4 \mathrm{com} \mathrm{V}_{\mathrm{DS}}=3 \mathrm{~V}$ em $\mathrm{V}_{\mathrm{GT}}=0,25 \mathrm{~V}$ tem aproximadamente $129 \%$ e em $\mathrm{V}_{\mathrm{GT}}=$ $2,8 \mathrm{~V}$ tem aproximadamente $2,65 \%$. O desvio relativo em inversão forte é menor do que a variação imposta na concentração de dopantes.

Foi traçada a curva do desvio relativo da transcondutância em função da sobretensão de condução com polarização de dreno de $50 \mathrm{mV}, 1.5 \mathrm{~V}$ e $3 \mathrm{~V}$, os resultados são apresentados conforme apresentado Figura 43. 
Figura 43 - Desvio relativo da transcondutância em função da sobretensão de condução de $\mathrm{V}_{\mathrm{DS}}=50 \mathrm{mV}(\mathrm{A}), 1,5 \mathrm{~V}(\mathrm{~B})$ e $3 \mathrm{~V}(\mathrm{C})$, com concentração de $5.10^{16} \mathrm{~cm}^{-3}$ variando $\pm 10 \%$.
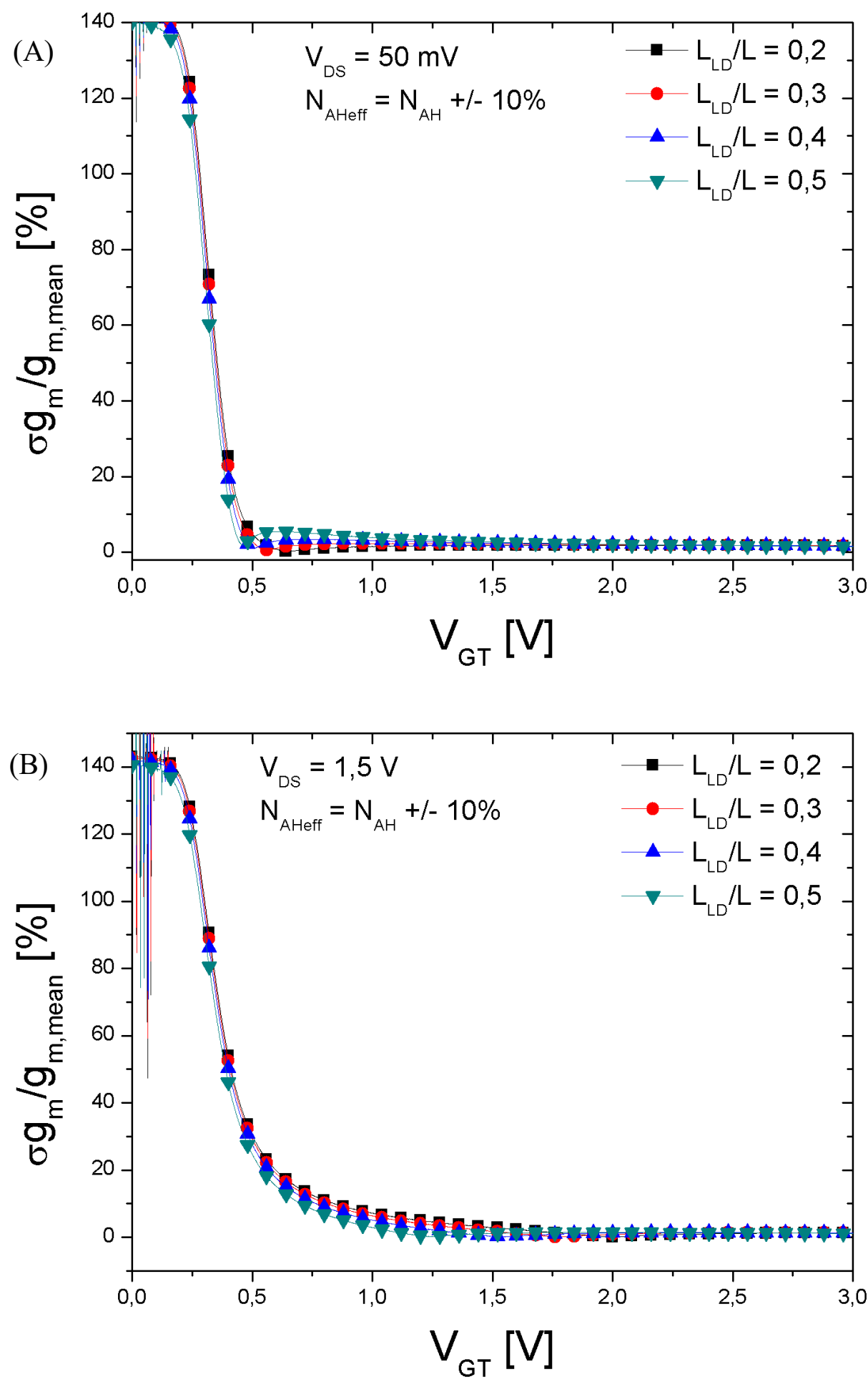


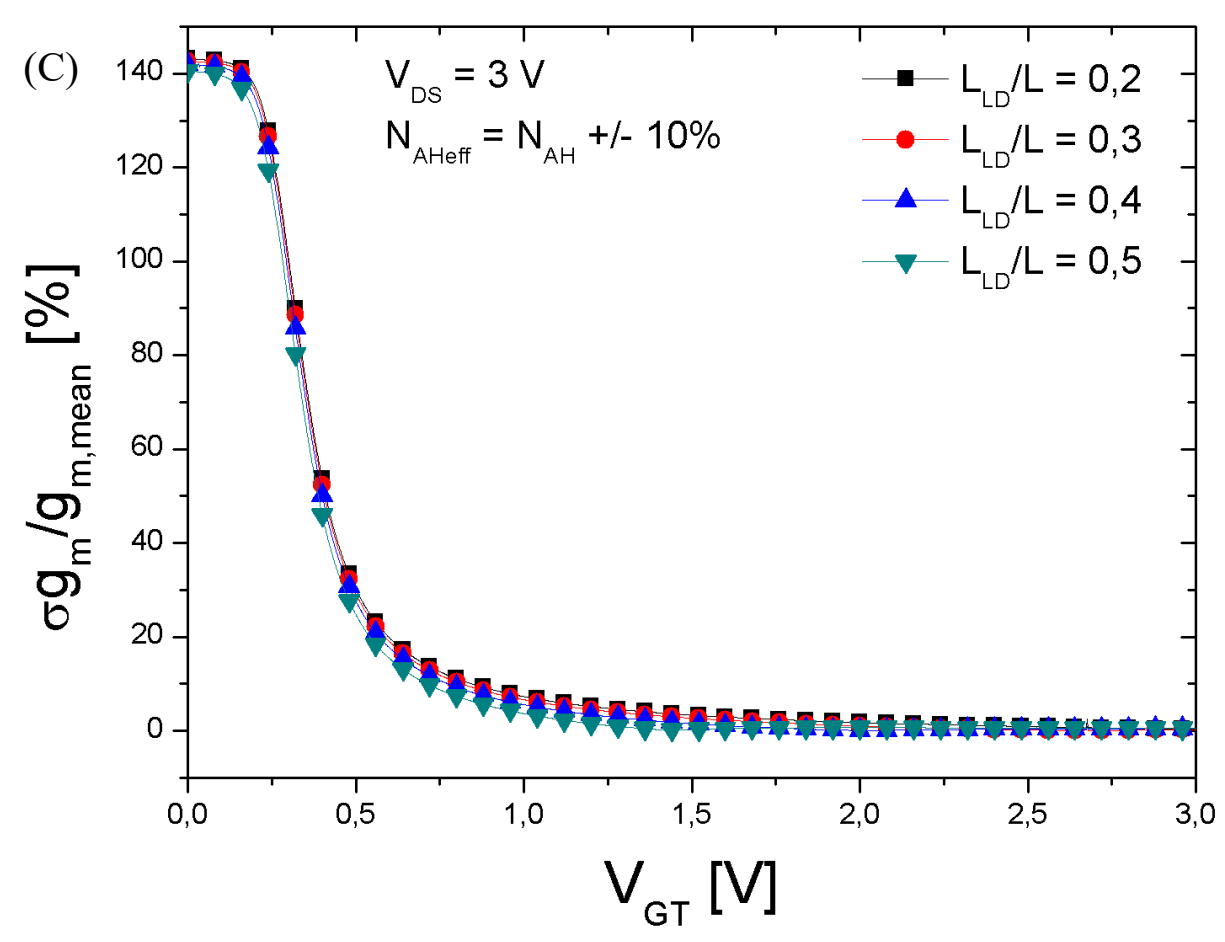

Fonte: Alves, 2017.

Ao se variar a concentração de dopantes o desvio relativo aumenta em relação aos desvios obtidos com a variação do comprimento efetivo de canal. Previamente foi utilizado o exemplo de $\mathrm{L}_{\mathrm{LD}} / \mathrm{L}=0,5 \mathrm{em} \mathrm{V}_{\mathrm{GT}}=2,5 \mathrm{~V}$ variando-se a comprimento efetivo de canal, o qual apresentou os desvios relativos de $0,44 \%, 0,35 \%$ e $0,13 \%$ para $\mathrm{V}_{\mathrm{DS}}$ de $50 \mathrm{mV}, 1,5 \mathrm{~V}$ e $3 \mathrm{~V}$, respectivamente. Para a variação da concentração de dopantes no mesmo ponto tem-se os desvios de $1,72 \%, 1,22 \%$ e $0,53 \%$, respectivamente. Conforme aumentamos a polarização de dreno o desvio relativo aumenta, como exemplo a razão $\mathrm{L}_{\mathrm{LD}} / \mathrm{L}=0,3 \mathrm{em} \mathrm{V}_{\mathrm{GT}}=1,5 \mathrm{~V}$ tem aproximadamente $1,73 \%$ com $\mathrm{V}_{\mathrm{DS}}$ de $1,5 \mathrm{~V}$ e aproximadamente $2,62 \%$ com $\mathrm{V}_{\mathrm{DS}}$ de $3 \mathrm{~V}$.

A partir dos resultados calculados, foram extraídos os valores médios e desvios relativos da transcondutância para $\mathrm{V}_{\mathrm{GT}}=200 \mathrm{mV}$ e $\mathrm{V}_{\mathrm{DS}}=1,5 \mathrm{~V}$, mostrado na Tabela 19 . Pode-se observar que a variação imposta na concentração de dopantes é atenuada no desvio relativo da transcondutância, como exemplo a razão $\mathrm{L}_{\mathrm{LD}} / \mathrm{L}=0,2$ apresentou um desvio relativo da transcondutância de $1,82 \%$. 
Tabela 19 - Valor médio e desvio relativo da transcondutância para GC SOI MOSFETs, extraído para $\mathrm{V}_{\mathrm{GT}}=200 \mathrm{mV}$ e $\mathrm{V}_{\mathrm{DS}}=1,5 \mathrm{~V}$ com concentração de $5.10^{16} \mathrm{~cm}^{-3}$ variando $\pm 10 \%$.

\begin{tabular}{ccc}
\hline $\mathbf{L} \mathbf{L D} / \mathbf{L}$ & $\mathrm{g}_{\mathrm{m}, \text { mean }}[\mu \mathrm{S}]$ & $\sigma \mathrm{g}_{\mathrm{m}} / \mathrm{g}_{\mathrm{m}, \text { mean }}[\%]$ \\
\hline $\mathbf{0 , 2}$ & 7,59 & 1,82 \\
\hline $\mathbf{0 , 3}$ & 8,68 & 1,44 \\
\hline $\mathbf{0 , 4}$ & 10,23 & 0,86 \\
\hline $\mathbf{0 , 5}$ & 12,68 & 0,48
\end{tabular}

Fonte: Alves, $20 \overline{17 .}$

Em posse dos valores da tensão de limiar para cada dispositivo, foi simulada a curva $\mathrm{I}_{\mathrm{DS}}$ em função de $\mathrm{V}_{\mathrm{DS}}$ com sobretensão de condução de $0 \mathrm{mV}, 200 \mathrm{mV}, 400 \mathrm{mV}, 600 \mathrm{mV}$ e $800 \mathrm{mV}$. Como exemplo, é apresentada a curva IDS em função de $\mathrm{V}_{\mathrm{DS}}$ com sobretensão de condução de $600 \mathrm{mV}$ na Figura 44.

Figura 44 - Curva $\mathrm{I}_{\mathrm{DS}}$ em função de $\mathrm{V}_{\mathrm{DS}}$ com sobretensão de condução $\mathrm{V}_{\mathrm{GT}}=600 \mathrm{mV}$ com concentração de $5.10^{16} \mathrm{~cm}^{-3}$ variando $\pm 10 \%$.

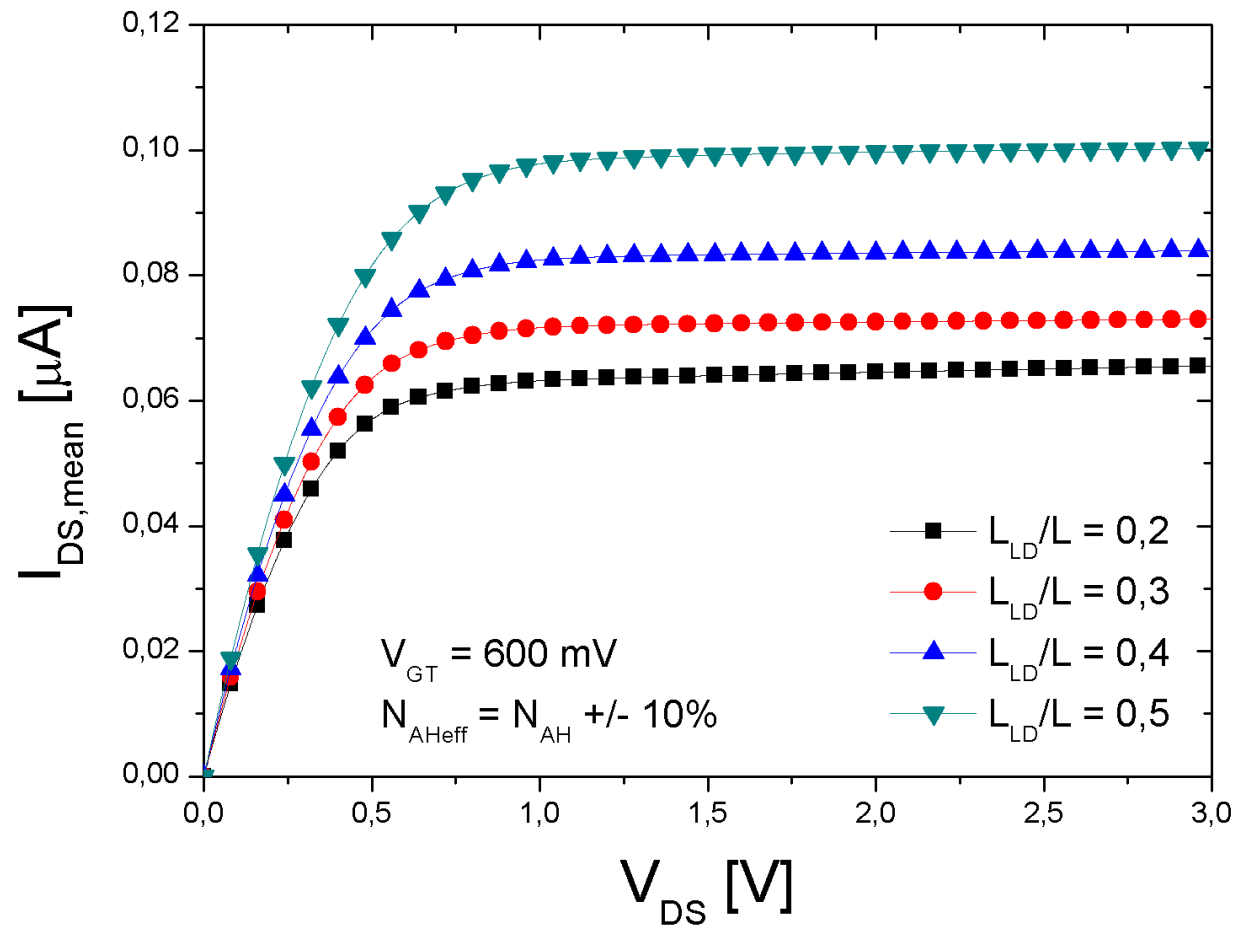

Fonte: Alves, 2017. 
Foi extraído o desvio relativo da corrente de dreno em função da tensão de dreno, conforme apresentado na Figura 45.

Figura 45 - Desvio relativo da corrente de dreno em função de $V_{D S}$ com sobretensão de condução $\mathrm{V}_{\mathrm{GT}}=0 \mathrm{mV}$ (A), $200 \mathrm{mV}$ (B), $400 \mathrm{mV}$ (C) e $600 \mathrm{mV}$ (D), com concentração de $5.10^{16} \mathrm{~cm}^{-3}$ variando $\pm 10 \%$.

(A)

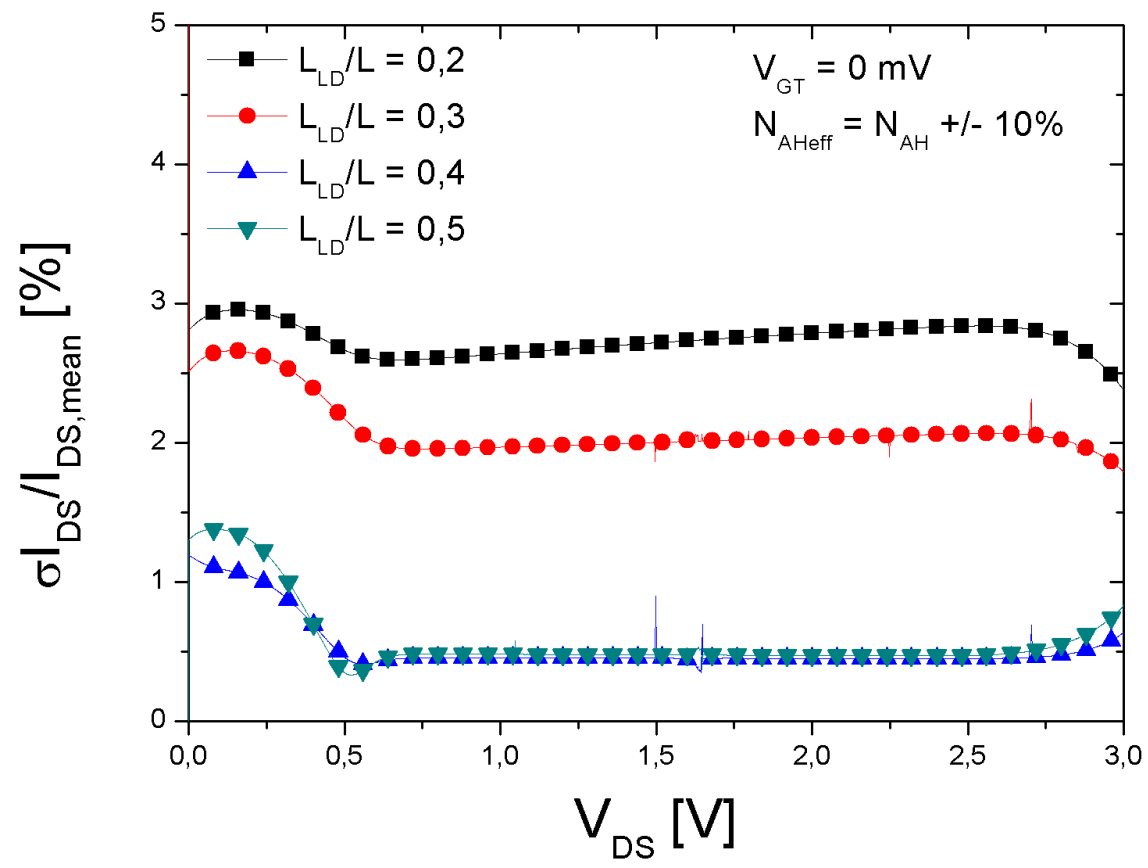

(B)

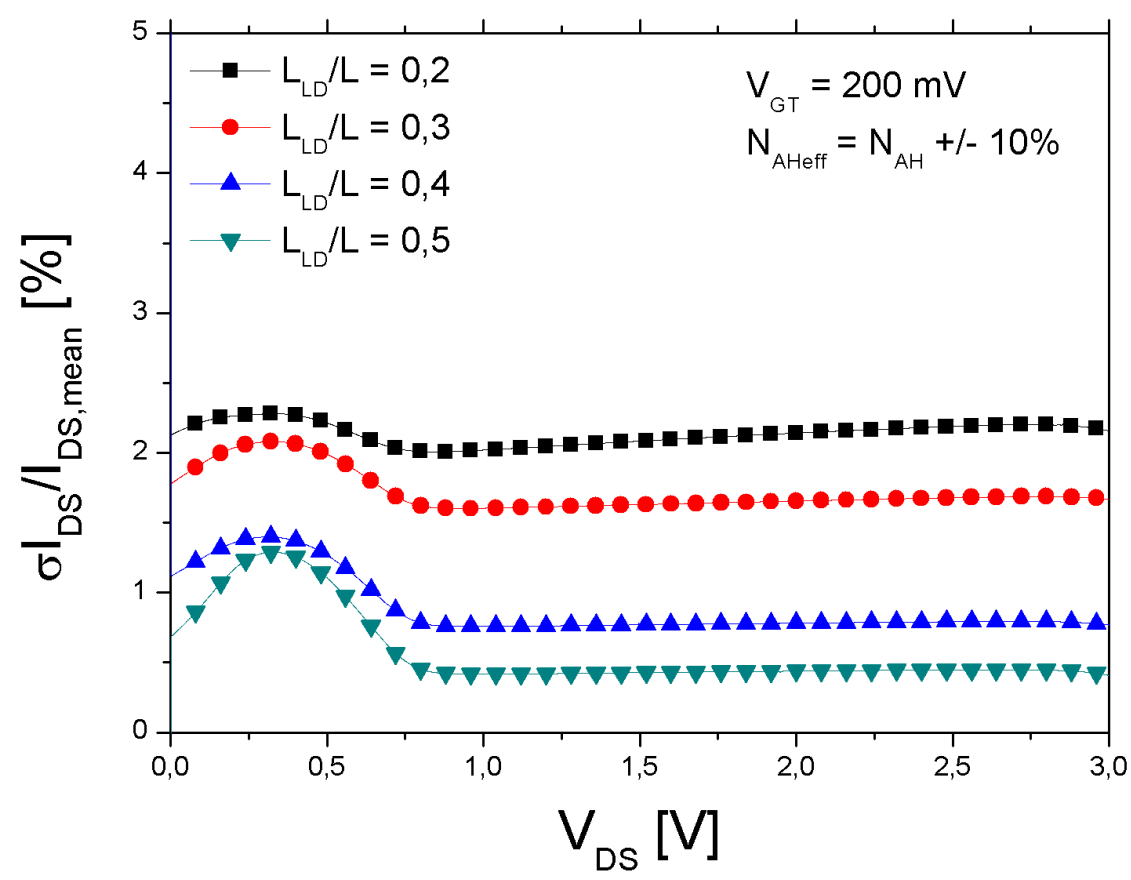


(C)

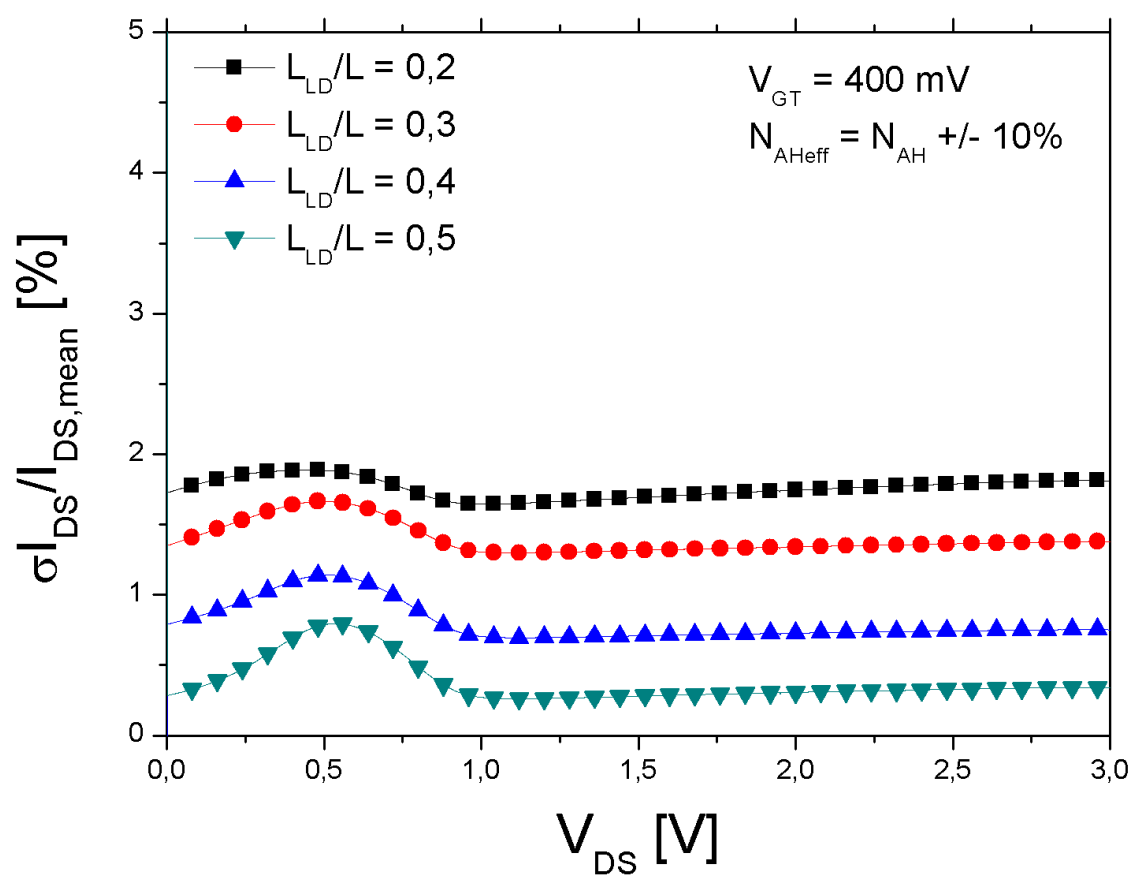

(D)

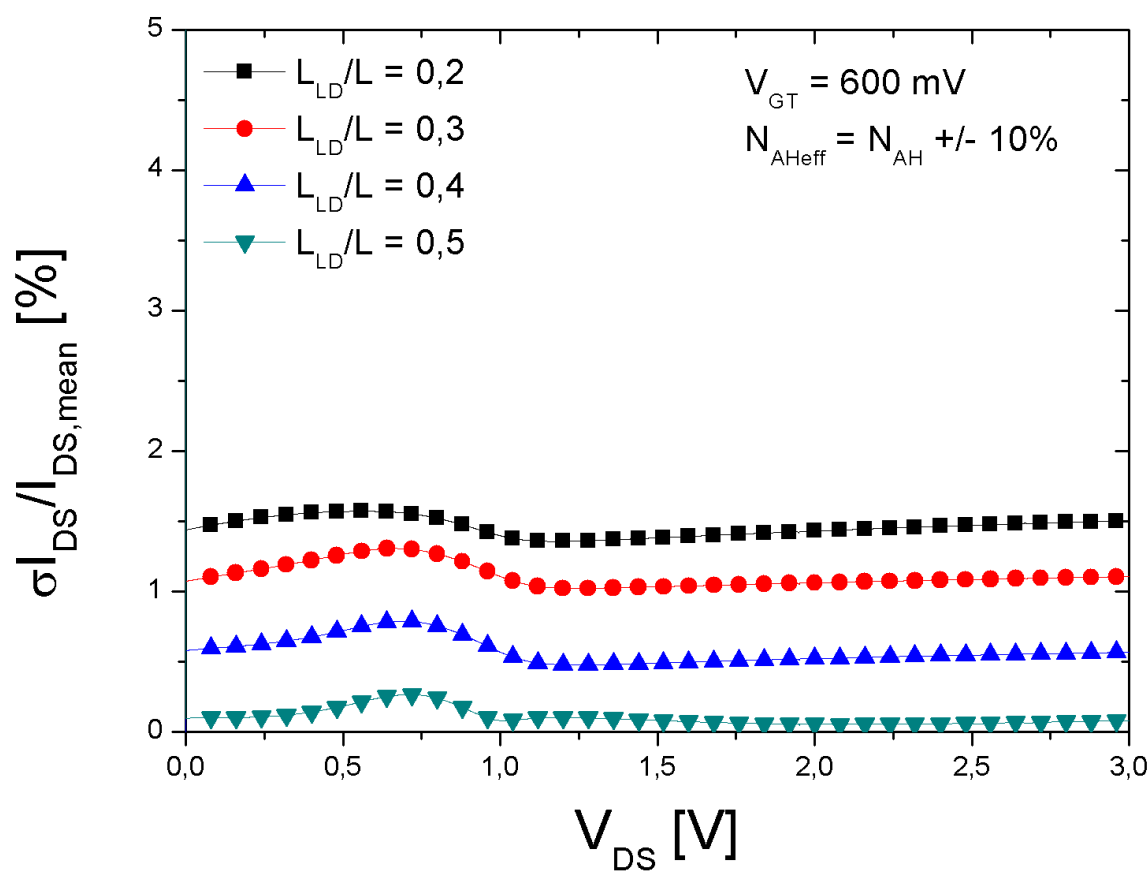

Fonte: Alves, 2017.

Ao se variar a concentração de dopantes, o desvio relativo da corrente de dreno diminui em relação aos desvios relativos obtidos com a variação do comprimento efetivo de canal. Previamente foi utilizado o exemplo de $\mathrm{L}_{\mathrm{LD}} / \mathrm{L}=0,3 \mathrm{em} \mathrm{V}_{\mathrm{GT}}$ de $200 \mathrm{mV}$ e $600 \mathrm{mV}$ variando-se a comprimento efetivo de canal, o qual apresentou os desvios relativos de $2 \%$ e 
$1,8 \%$, respectivamente. Para a variação da concentração de dopantes no mesmo ponto os desvios foram de $1,6 \%$ e $1,06 \%$, respectivamente.

Ao se variar o comprimento efetivo do canal, conforme aumentamos a razão $\mathrm{L}_{\mathrm{LD}} / \mathrm{L}, \mathrm{o}$ desvio relativo da corrente de dreno aumenta. Por outro lado, ao se variar a concentração de dopantes, conforme aumentamos a razão $\mathrm{L}_{\mathrm{LD}} / \mathrm{L}$ o desvio relativo da corrente de dreno diminui. A variação do comprimento efetivo do canal e da concentração de dopantes seguem tendências opostas, o que faz com que o desvio relativo da corrente de dreno seja atenuado.

A partir das curvas de corrente, foram extraídos os valores de condutância de dreno para cada dispositivo e calculado o valor médio e seu desvio relativo, conforme pode ser observado na Tabela 20.

Tabela 20 - Valor médio e desvio relativo da condutância de dreno para GC SOI MOSFETs, extraídos para $\mathrm{V}_{\mathrm{GT}}=200 \mathrm{mV}$ e $\mathrm{V}_{\mathrm{DS}}=1,5 \mathrm{~V}$ com concentração de $5.10^{16} \mathrm{~cm}^{-3}$ variando \pm $10 \%$.

\begin{tabular}{|c|c|c|}
\hline $\mathbf{L}_{\mathbf{L D}} / \mathbf{L}$ & $\mathrm{g}_{\mathrm{D}, \text { mean }}[\mathrm{nS}]$ & $\sigma \mathrm{g}_{\mathrm{D}} / \mathrm{g}_{\mathrm{D}, \text { mean }}[\%]$ \\
\hline 0,2 & 21,02 & 7,40 \\
\hline 0,3 & 8,37 & 8,14 \\
\hline 0,4 & 4,13 & 8,30 \\
\hline 0,5 & 4,29 & 9,62 \\
\hline
\end{tabular}

Fonte: Alves, $20 \overline{17 .}$

Pode-se observar que apesar do valor médio da condutância de dreno não variar muito quando comparado com os valores extraídos variando o comprimento de canal efetivo, o desvio relativo acaba sendo, de forma geral, menor do que os valores previamente encontrados. O desvio relativo acaba sendo menor do que a variação imposta, que é de $10 \%$.

Foram calculados os valores de tensão Early, com sobretensão de condução de 200 $\mathrm{mV}$, conforme apresentado na Figura 46. 
Figura 46 - Tensão Early em função da tensão de dreno com sobretensão de condução $\mathrm{V}_{\mathrm{GT}}=$ $200 \mathrm{mV}$ com concentração de $5.10^{16} \mathrm{~cm}^{-3}$ variando $\pm 10 \%$.

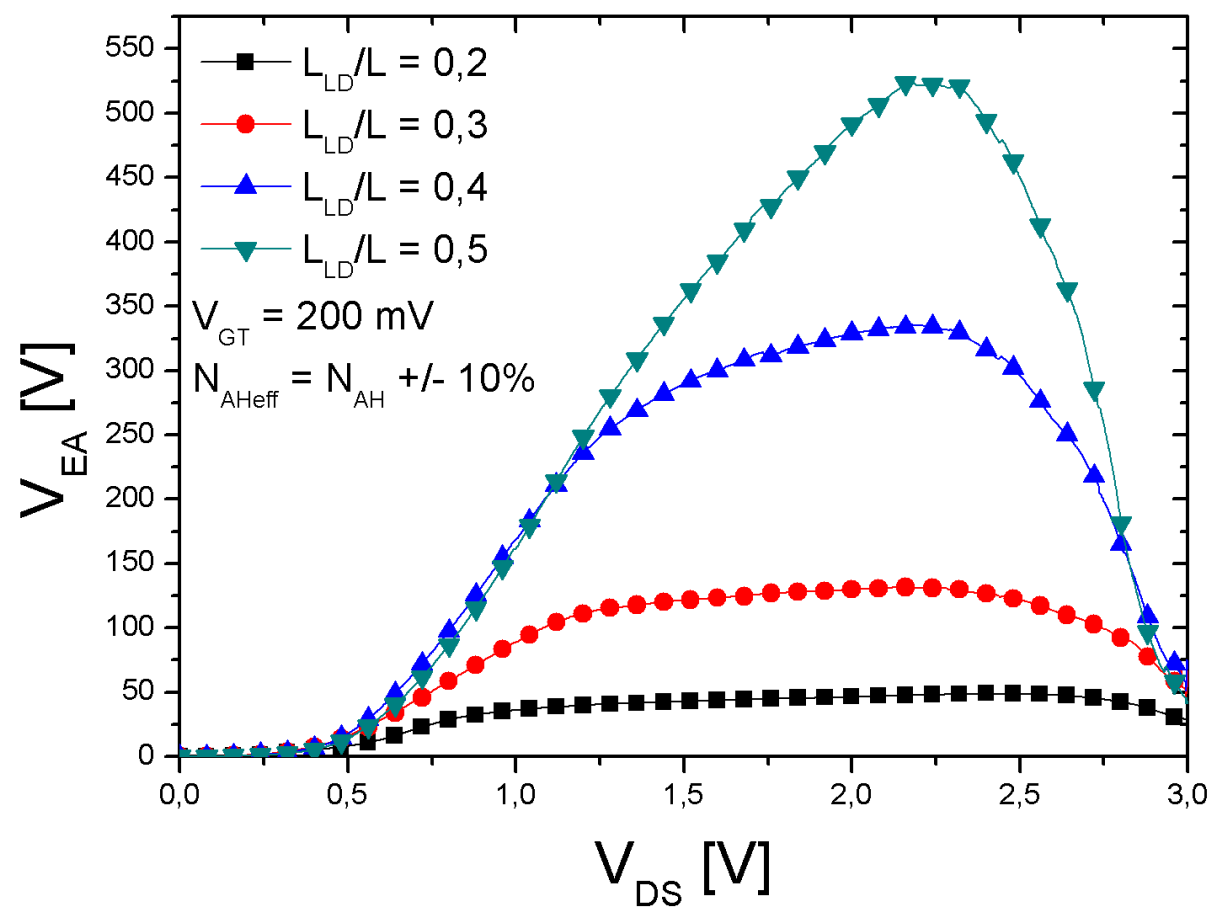

Fonte: Alves, 2017.

Para a tensão de dreno de 1,5 V foram extraídos os valores da tensão Early conforme Tabela 21.

Tabela 21 - Tensão Early e desvio relativo dos GC SOI MOSFETs com $\mathrm{V}_{\mathrm{GT}}=200 \mathrm{mV}$ e $\mathrm{V}_{\mathrm{DS}}$ $=1,5 \mathrm{~V}$ com concentração de $5.10^{16} \mathrm{~cm}^{-3}$ variando $\pm 10 \%$.

\begin{tabular}{ccc}
\hline $\mathbf{L} \mathbf{L D} / \mathbf{L}$ & $\mathrm{V}_{\mathrm{EA}, \text { mean }}[\mathrm{V}]$ & $\sigma \mathrm{V}_{\mathrm{EA}} / \mathrm{V}_{\mathrm{EA}, \text { mean }}[\%]$ \\
\hline $\mathbf{0 , 2}$ & 42,8 & 5,34 \\
\hline $\mathbf{0 , 3}$ & 121,3 & 6,92 \\
\hline $\mathbf{0 , 4}$ & 289,5 & 7,76 \\
\hline $\mathbf{0 , 5}$ & 355,7 & 9,05
\end{tabular}

Fonte: Alves, $20 \overline{17 .}$

Pode-se observar uma melhora na tensão Early média quando varia-se o comprimento efetivo do canal, como exemplo a razão $\mathrm{L}_{\mathrm{LD}} / \mathrm{L}=0,2$ que apresenta $\mathrm{V}_{\mathrm{EA} \text {,mean }}=516,9 \mathrm{~V}$. Porém uma diminuição do desvio relativo da tensão Early quando variamos a concentração de dopantes, como exemplo a razão $\mathrm{L}_{\mathrm{LD}} / \mathrm{L}=0,2$ que apresenta $17,4 \%$ quando variado o 
comprimento efetivo de canal. O desvio relativo da tensão Early está abaixo da variação provocada, que é de $10 \%$.

Foi calculado a ganho de tensão $\left(\mathrm{A}_{\mathrm{V}}=\mathrm{g}_{\mathrm{m}} / \mathrm{g}_{\mathrm{D}}\right)$ para $\mathrm{V}_{\mathrm{GT}}=200 \mathrm{mV}$ e $\mathrm{V}_{\mathrm{DS}}=1,5 \mathrm{~V}$, para cada dispositivo, o ganho de tensão médio e o desvio relativo do ganho de tensão estão presentes na Tabela 22 .

Tabela 22 - Ganho de tensão e desvio relativo dos GC SOI MOSFETs com $\mathrm{V}_{\mathrm{GT}}=200 \mathrm{mV}$ e $\mathrm{V}_{\mathrm{DS}}=1,5 \mathrm{~V}$ concentração de $5.10^{16} \mathrm{~cm}^{-3}$ variando $\pm 10 \%$.

\begin{tabular}{ccc}
\hline $\mathbf{L}_{\mathbf{L D}} / \mathbf{L}$ & $\mathrm{A}_{\mathrm{V} \text {,mean }}[\mathrm{V} / \mathrm{V}]$ & $\sigma \mathrm{A}_{\mathrm{V}} / \mathrm{A}_{\mathrm{V}, \text { mean }}[\%]$ \\
\hline $\mathbf{0 , 2}$ & 362,3 & 5,58 \\
\hline $\mathbf{0 , 3}$ & 1041,9 & 6,68 \\
\hline $\mathbf{0 , 4}$ & 2487,4 & 7,38 \\
\hline $\mathbf{0 , 5}$ & 2973,6 & 9,09
\end{tabular}

Fonte: Alves, $20 \overline{17 .}$

Pode-se observar uma melhora no ganho de tensão médio quando variamos a concentração de dopantes, como exemplo a razão $\mathrm{L}_{\mathrm{LD}} / \mathrm{L}=0,3$ que apresenta $127,69 \mathrm{~V} / \mathrm{V}$. E também uma melhora do desvio relativo quando variado a concentração de dopantes, como exemplo a razão $\mathrm{L}_{\mathrm{LD}} / \mathrm{L}=0,3$ que apresenta $14,09 \%$.

O desvio relativo do ganho de tensão, da tensão de limiar e da transcondutância acaba sendo maior que a variação imposta na concentração de dopantes, porém o desvio relativo da inclinação de sublimiar, da condutância de saída e da tensão Early acaba sendo menor que a variação imposta. 


\section{CONCLUSÕES}

Inicialmente, neste trabalho de Mestrado, foi realizado um estudo teórico dos assuntos necessários para seu desenvolvimento. Após esse processo foi dado início às etapas de caracterização elétrica, simulações numéricas e tratamento de dados, com o intuito de comparar os resultados obtidos com os esperados teoricamente.

Foi estudado, através de medidas experimentais, o descasamento GC SOI MOSFETs com diferentes razões de $\mathrm{L}_{\mathrm{LD}} / \mathrm{L}$, variando as condições de polarização, a fim de analisar as variações dos parâmetros de saída, como tensão de limiar, corrente, transcondutância, condutância de dreno, tensão Early e ganho de tensão.

Através das curvas obtidas foi possível observar que a tensão de limiar é altamente influenciada pela concentração de dopantes e é o fator que mais influência no descasamento de transistores operando com baixas tensões de porta. Ao se aumentar a tensão de porta, o desvio tanto da corrente como da transcondutância diminuem, uma vez que o descasamento da tensão de limiar se torna menos significativo.

Foi observado que GC SOI MOSFETs apresentam maiores valores de corrente de dreno do que os transistores convencionais. Esses valores aumentam conforme aumentamos a razão $\mathrm{L}_{\mathrm{LD}} / \mathrm{L}$, porém o desvio também aumenta devido à diminuição do comprimento efetivo. Ao se aumentar a tensão de porta o desvio passa a ser influenciado primordialmente pelo fator de corrente, que sofre pouca variação. Portanto, o transistor operando em inversão fraca apresenta maior desvio relativo da transcondutância, e conforme aumenta-se a tensão de porta aplicada esse desvio tende a um valor a $1 \%$.

Quando a razão $\mathrm{L}_{\mathrm{LD}} / \mathrm{L}$ é aumentada, o comprimento efetivo de canal diminui e a corrente de dreno de saturação aumenta, e a condutância de saída é reduzida pela presença da região fracamente dopada, consequentemente, aumentando a tensão Early.

A menor condutância de saída associada ao aumento da transcondutância em transistores de canal gradual em relação aos transistores convencionais faz com que haja um aumento do ganho de tensão, porém um aumento do desvio relativo.

Foram realizadas simulações numéricas bidimensionais, impondo variações no comprimento e na concentração de dopantes da região fortemente dopada, com o objetivo de avaliar o impacto de cada uma destas variáveis sobre as características elétricas dos transistores de canal gradual.

As simulações variando o comprimento efetivo do canal em $\pm 30 \mathrm{~nm}$ e a concentração de dopantes do canal em $\pm 10 \%$ mostraram que ambos os parâmetros influenciam no 
descasamento dos transistores. A variação da concentração dos dopantes tem maior impacto no desvio relativo da tensão de limiar. Pode-se concluir então que o desvio relativo da tensão de limiar deve-se principalmente devido à flutuação da concentração de dopantes na região fortemente dopada.

Os outros parâmetros analisados sofrem maior influência da variação do comprimento efetivo do canal, como exemplo a razão $\mathrm{L}_{\mathrm{LD}} / \mathrm{L}=0,3$ que apresenta um desvio relativo de $13,9 \%$ na tensão Early e 14,09\% no ganho de tensão quando variado o comprimento efetivo de canal e 6,92\% e 6,68\%, respectivamente, quando variado a concentração de dopantes.

A tendência do desvio relativo dos parâmetros analisados, ao se aplicar a variação do comprimento efetivo de canal e da concentração de dopantes, nem sempre segue a mesma tendência de aumento ou diminuição do desvio relativo. O que nos leva a concluir que caso haja a aplicação de ambas variações, essas tendências opostas acabam fazendo com que o desvio relativo acabe ficando em um meio termo. Como exemplo tem-se a condutância de dreno, o qual apresenta um desvio relativo maior do que a variação imposta no comprimento efetivo, porém um menor desvio relativo do que a variação imposta na concentração de dopantes.

Contudo, essa variação da concentração de dopantes é bem controlada pelos processos de fabricação. Já o controle da difusão dos dopantes entre a região altamente dopada e fracamente dopada não é tão precisa, principalmente em comprimentos de canais efetivos menores.

Apesar do aumento do desvio relativo nos GC SOI MOSFETs o aumento do ganho de tensão e da tensão Early em relação à dispositivos SOI convencionais pode acabar compensando o aumento do desvio relativo.

Como continuação do trabalho, pode-se simular estruturas que levam em consideração variações de outros parâmetros, tais como o comprimento total do canal juntamente com a variação do $\mathrm{L}_{\mathrm{LD}}$, além da inclusão de efeitos como a variação da temperatura. 


\section{REFERÊNCIAS}

ASSALTI, R. Influência de parâmetros tecnológicos e geométricos sobre o desempenho de transistores SOI de Canal Gradual. Tese (Mestrado em Engenharia Elétrica) - Centro Universitário da FEI, São Bernardo do Campo, 2015.

BREWS, J. R. Subthreshold behaviour of uniformly and nonuniformly doped long-channel MOSFET. IEEE Transactions on Electron Devices, v. 26, n. 9, p. 1282-1291, 1979. Disponível em: <http://ieeexplore.ieee.org/document/1480172/>. Acesso em: 20 jan. 2016.

CERDEIRA, A. et al. Advantages of graded-channel SOI FD MOSFET for applications as a quasi-linear resistor. IEEE Trans on Electron Devices, v.52, n.5, p.967-972, 2005.

Disponível em: <http://ieeexplore.ieee.org/document/1424387/>. Acesso em: 16 jan. 2016.

CHOI, J.Y.; FOSSUM, J.G. Analysis and control of floating-body bipolar effects in fully depletes submicrometer SOI MOSFETs. IEEE Transactions on Electron Devices, v.38, n.6, p.1384-1391, 1991. Disponível em: <http://ieeexplore.ieee.org/document/81630/>. Acesso em: 15 jan. 2016.

CHUANG, C.T. et al. SOI for digital CMOS VLSI: design considerations and advances. Proceedings of the IEEE, v. 86, n.4, p. 689-720, 1998. Disponível em: $<$ http://ieeexplore.ieee.org/document/663545/>. Acesso em: 20 jan. 2016.

COLINGE, J. P. Fully-Depleted SO1 CMOS for Analog Applications. IEEE Transactions on Electron Devices, vol. 45, n. 5, p. 1010-1016, 1998. Disponível em: $<$ http://ieeexplore.ieee.org/document/669511/>. Acesso em: 15 jan. 2016

COLINGE, J.P.; COLINGE, C.A. Physics of semiconductor devices. 1st ed. Massachusetts: Kluwer Academic, 2002.

COLINGE, J.P. Silicon-On-Insulator Technology: Materials to VLSI. 3rd ed. Massachusetts: Kluwer Academic, 2004.

CROON, J.A. et al. An easy-to-use mismatch model for the MOS transistor. IEEE Journal of Solid-State Circuits, v.37, p. 1056-1064, 2002. Disponível em:

$<$ http://ieeexplore.ieee.org/document/1020245/>. Acesso em: 16 jan. 2016.

CROON, J. A. et al. Physical modeling and prediction of the matching properties of MOSFETs. Proceeding of the 34th European Solid-State Device Research Conference, p. 193-196, 2004. Disponível em: <http://ieeexplore.ieee.org/document/1356522/>. Acesso em: 16 jan. 2016.

DIFRENZA, R.; LLINARES, P.; GHIBAUDO, G.. A new model for the current factor mismatch in the MOS transistor. Solid-State Electronics, v.47, n.7, p. 1161-1171, 2003. Disponível em: 
$<$ https://www.researchgate.net/publication/238925601_A_new_model_for_the_current_factor mismatch_in_the_MOS_transistor>. Acesso em: 16 jan. 2016.

FLANDRE, D. et al; Comparison of SOI versus bulk performances of CMOS micropower single-stage OTAs. Electronics Letters, v.30, n.23, p.1933-1934, 1994. Disponível em: $<$ http://ieeexplore.ieee.org/document/335665/>. Acesso em: 16 jan. 2016.

FLANDRE, D. et al. Modelling and application of fully depleted SOI MOSFETs for low voltage, low power analogue CMOS circuits. Solid-State Electronics, v.39, n. 4, p. 455-460, 1996. Disponível em:

$<$ http://www.sciencedirect.com/science/article/pii/0038110195001670>. Acesso em: 16 jan. 2016.

GROESENEKEN, G. et al. Temperature dependence of threshold voltage in thin-film SOI MOSFETs. IEEE Electron Device Letters, v.11, n.8, p. 329-331, 1990. Disponível em: $<$ http://ieeexplore.ieee.org/document/57923/>. Acesso em: 16 jan. 2016.

GUTIERREZ, E. A.; DEEN, J; CLAEYS, C. L. Low temperature electronics: physics, devices, circuits and applications. San Diego: Academic Press, 1991.

JENG, M. C. et al. The effects of source/drain resistance on deep submicrometer device performance. IEEE Transactions on Electron Devices, v.37, n.11, p. 2408-2410, 1990. Disponível em: <http://ieeexplore.ieee.org/document/62301/>. Acesso em: 16 jan. 2016.

KINGET, P. R. Device mismatch and tradeoffs in the design of analog circuits. IEEE Journal of Solid-State Circuits, vol. 40, p. 1212-1224, 2005. Disponível em: $<\mathrm{http}$ ://ieeexplore.ieee.org/document/1435599/>. Acesso em: 16 jan. 2016.

KISTLER, N; et al. Sub-quarter-micrometer CMOS on ultrathin (400 ̊) SOI. IEEE Electronic Devices Letters, v.13, p.235-237, 1992. Disponível em:

$<$ http://ieeexplore.ieee.org/document/145038/>. Acesso em: 16 jan. 2016.

KISTLER, N.; WOO, J. Detailed characterization and analysis of the breakdown voltage in fully depleted SOI n-MOSFET's. IEEE Transactions on Electron Devices, v.41, n.7, p.1217-1221, 1994. Disponível em: $<$ http://ieeexplore.ieee.org/document/293350/>. Acesso em: 09 fev. 2016.

KRISHNAN, S.; FOSSUM, J.G. Grasping SOI floating-body effects. IEEE Circuits and Devices Magazine, v.14, n.4, p. 32-37, 1998. Disponível em: $<\mathrm{http}$ ://ieeexplore.ieee.org/abstract/document/708479/>. Acesso em: 10 fev. 2016.

LAKER, K. R.; SANSEN, W. M. C. Design of analog integrated circuits and systems. New York: McGraw-Hill, 1994. 
LIM, H.K.; FOSSUM, J.G. Threshold voltage of thin-film silicon-on-insulator (SOI) MOSFET's. IEEE Transactions on Electron Devices, v.30, p.1244-1251, 1983. Disponível em: $<$ http://ieeexplore.ieee.org/document/1483183/>. Acesso em: 20 jan. 2016.

LIM, H.K.; FOSSUM, J.G. Current-voltage characteristics of thin-film SOI MOSFET's in strong inversion. IEEE Transactions on Electron Devices, v.31, p.401-408, 1984.

Disponível em: <http://ieeexplore.ieee.org/document/1483825/>. Acesso em 1 mar. 2016.

MARTINO, J. A., PAVANELLO, M. A., VERDONCK , P. B. Caracterização Elétrica de Tecnologia e Dispositivos MOS. $1^{\mathrm{a}}$ ed. Thomson, 2004.

MULLER, R. S.; KAMINS, T. I. Device electronics for integrated circuits. $2^{\text {nd }}$ ed. New York: Wiley, 1986.

ORTIZ-CONDE, A. et al. A review of recent MOSFET threshold voltage extraction methods. Microelectronics Reliability, v.42, n.4-5, p. 583-596, 2002. Disponível em:

$<$ http://www.sciencedirect.com/science/article/pii/S0026271402000276>. Acesso em: 15 jan. 2016.

PAVANELLO, M.A. et al. The graded-channel SOI MOSFET to alleviate the parasitic bipolar effects and improve the output characteristics. In. SILICON-ON-INSULATOR TECHNOLOGY AND DEVICES, 9., 1999. Proceedings... Pennington: The Electrochemical Society, p.293-298, 1999.

PAVANELLO, M.A. et al. Analog performance and application of graded-channel fully depleted SOI MOSFETs. Solid-State Electronics, v.44, n.7, p.1219-1222, 2000. Disponível em: <http://www.sciencedirect.com/science/article/pii/S0038110100000344>. Acesso em: 15 jan. 2016.

PAVANELLO, M. A. Projeto, fabricação e caracterização elétrica de uma nova estrutura para SOI MOSFET, Tese (Doutorado em Engenharia Elétrica) - USP, São Paulo, 2000.

PAVANELLO, M. A. et al. An asymmetric channel SOI nMOSFET for reducing parasitic effects and improving output characteristics. Electrochemical and Solid-State Letters, v.1, p. 50-52, 2000. Disponível em: <http://esl.ecsdl.org/content/3/1/50.full.pdf >. Acesso em: 15 jan. 2016.

PAVANELLO, M. A.; MARTINO, J. A. ; FLANDRE, D. Graded-channel fully depleted silicon-on-insulator nMOSFET for reducing the parasitic bipolar effects. Solid-State Electronics, v.44, n.6, p.917-922, 2000. Disponível em: $<$ http://www.sciencedirect.com/science/article/pii/S0038110100000320>. Acesso em: 17 jan. 2016.

PELGROM, M. J.; TUINHOUT, H. P.; VERTREGT, M. Transistor matching in analog CMOS applications. International Electron Devices Meeting, p. 915-918, 1998. Disponível em: <http://ieeexplore.ieee.org/document/746503/>. Acesso em: 16 jan. 2016. 
SYNOPSYS. Sentaurus device user guide, 2010. Manual versão D-2010.03.

SAH, C. T. et al; Effect of zinc impurity in silicon solar-cell efficiency. IEEE Transaction on Electron Devices, v. 28, n. 3, p. 304-313, 1981. Disponível em:

$<\mathrm{http}$ ://ieeexplore.ieee.org/document/1481485/>. Acesso em: 20 jan. 2016.

SANCHEZ, J.J.; HSUEH, K.K.; DEMASSA, T.A. Drain-engineered hotelectronresistant device structures: a review. IEEE Transactions Electron Devices, v.36, n.6, p.1125-1132, 1989. Disponível em: <http://ieeexplore.ieee.org/document/24357/>. Acesso em: 17 jan. 2016.

SEDRA, A. S.; SMITH, K. C. Microeletrônica. $5^{\text {a }}$ ed. São Paulo: Pearson Prentice Hall, 2007.

SILVEIRA, F. et al; $\mathrm{A} \mathrm{g}_{\mathrm{m}} / \mathrm{I}_{\mathrm{D}}$ based methodology for the design of CMOS analog circuits and its application to the synthesis of a silicon-on-insulator micropower OTA. IEEE Journal of Solid-State Circuits, v.31, n.9, p.1314-1319, 1996. Disponível em:

$<$ http://ieeexplore.ieee.org/document/535416/>. Acesso em: 15 jan. 2016.

SOUZA, M. de. Desenvolvimento de um modelo analítico contínuo para transistores GC SOI nMOSFETs. Tese (Mestrado em Engenharia elétrica) - USP, São Paulo, 2005.

SOUZA, M. de; FLANDRE, D.; PAVANELLO, M. A. Study of matching properties of graded-channel SOI MOSFETs. Journal of Integrated Circuits and Systems, v. 3, n. 2, p. 69-75, 2008.

SOUZA, M. de. Modelagem simulação e fabricação de circuitos analógicos com transistores SOI convencionais e de canal gradual operando em temperaturas criogênicas. Tese (Doutorado em Engenharia Elétrica) - USP, São Paulo, 2008.

SZE, S.M. Physics of semiconductor devices. $2^{\text {nd }}$ ed. New York: John Wiley and Sons, 1981.

VANCAILLIE L. et al. MOSFET mismatch in weak/moderate inversion: model needs and implications for analog design. Proceedings of the European Solid-State Circuits Conference, p. 671-674, 2003. Disponível em:

$<$ http://ieeexplore.ieee.org/document/1257224/>. Acesso em: 18 jan, 2016.

VEERARAGHAVAN, S.; FOSSUM, J.G. A physical short-channel model for the thin-film SOI MOSFET applicable to device and circuit CAD. IEEE Transactions on Electron Devices, v.35, p. 1866-1875, 1988. Disponível em:

$<\mathrm{http}$ ://ieeexplore.ieee.org/abstract/document/7399/>. Acesso em: 20 jan. 2016.

VITTOZ, E.A. Low power design: ways to approach the limits. In: INTERNATIONAL SOLID-STATE CIRCUITS CONFERENCE (ISSCC), $41^{\text {st }}$, 1994, San Francisco. 
Proceedings... IEEE, p. 14-18, 1994. Disponível em:

$<$ http://ieeexplore.ieee.org/document/344744/>. Acesso em: 16 jan. 2016.

YOSHIMI, M. et al. Observation of mobility enhancement in ultrathin SOI MOSFETs.

Electronics Letters, v.24, n.17, p.1078-1079, 1988. Disponível em:

$<$ http://ieeexplore.ieee.org/document/191746/>. Acesso em: 15 jan. 2016.

YOSHIMI, M. et al. Two-dimensional simulation and measurement of high-performance MOSFETs made on a very thin SOI film. IEEE Transactions on Electron Devices, v.36, n.3, p.493-503, 1989. Disponível em: <http://ieeexplore.ieee.org/document/19959>. Acesso em: 14 fev. 2016.

YOUNG, K.K. Short-channel effect in fully depleted SOI MOSFETs. IEEE Transactions on Electron Devices. v.36, n.2, p.399-402, 1989. Disponível em:

$<$ http://ieeexplore.ieee.org/document/19942/>. Acesso em: 15 jan. 2016. 
APÊNDICE A - LAYOUT DOS CHIPS 
Layout MPC57 SET XX CHIP 2 OCT2008.

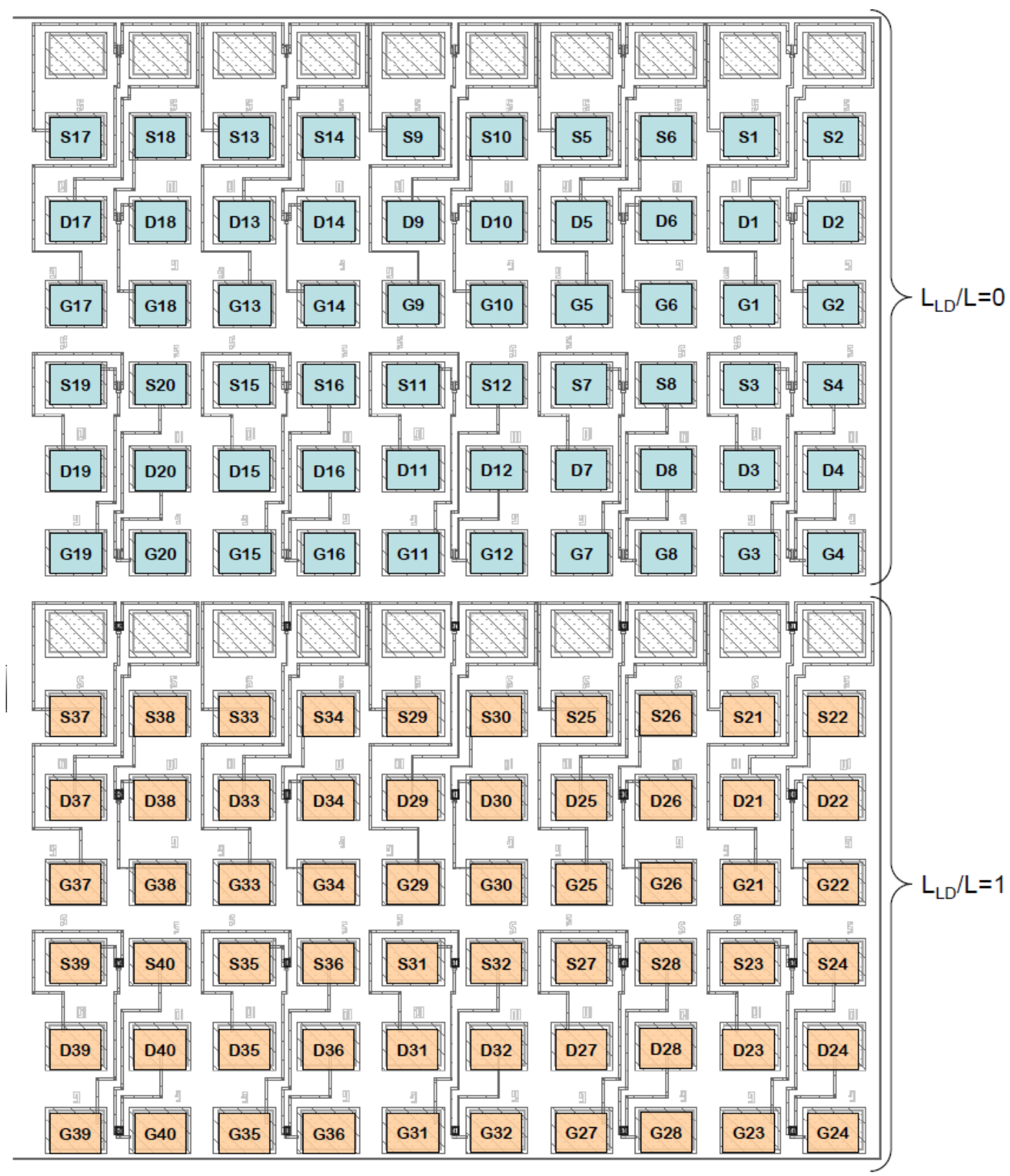


Layout MPC57 SET XX CHIP 13 OCT2008.

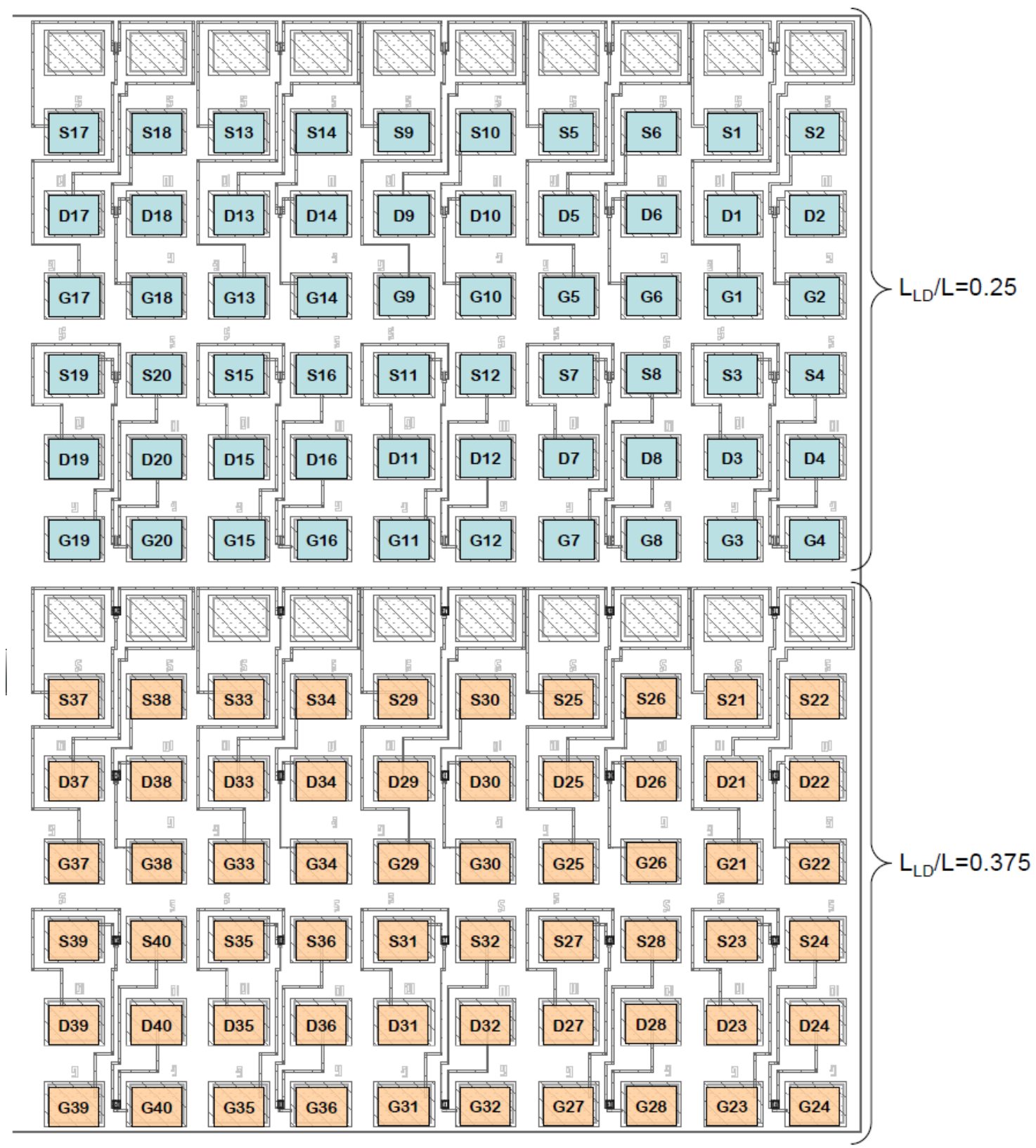


APÊNDICE B - ARQUIVOS SENTAURUS 
Arquivo para gerar um dispositivo de canal gradual no Sentaurus Strucutre.

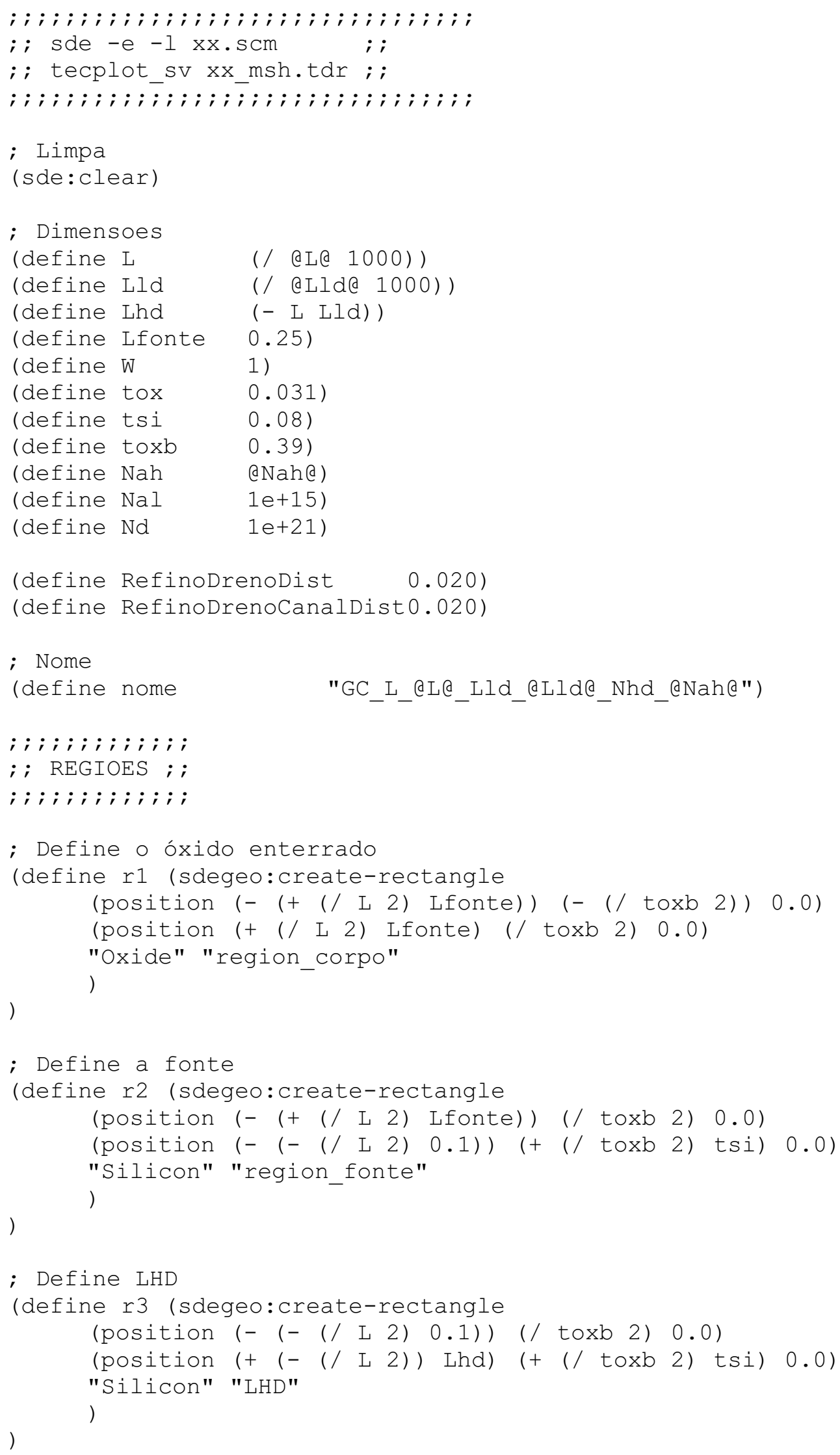




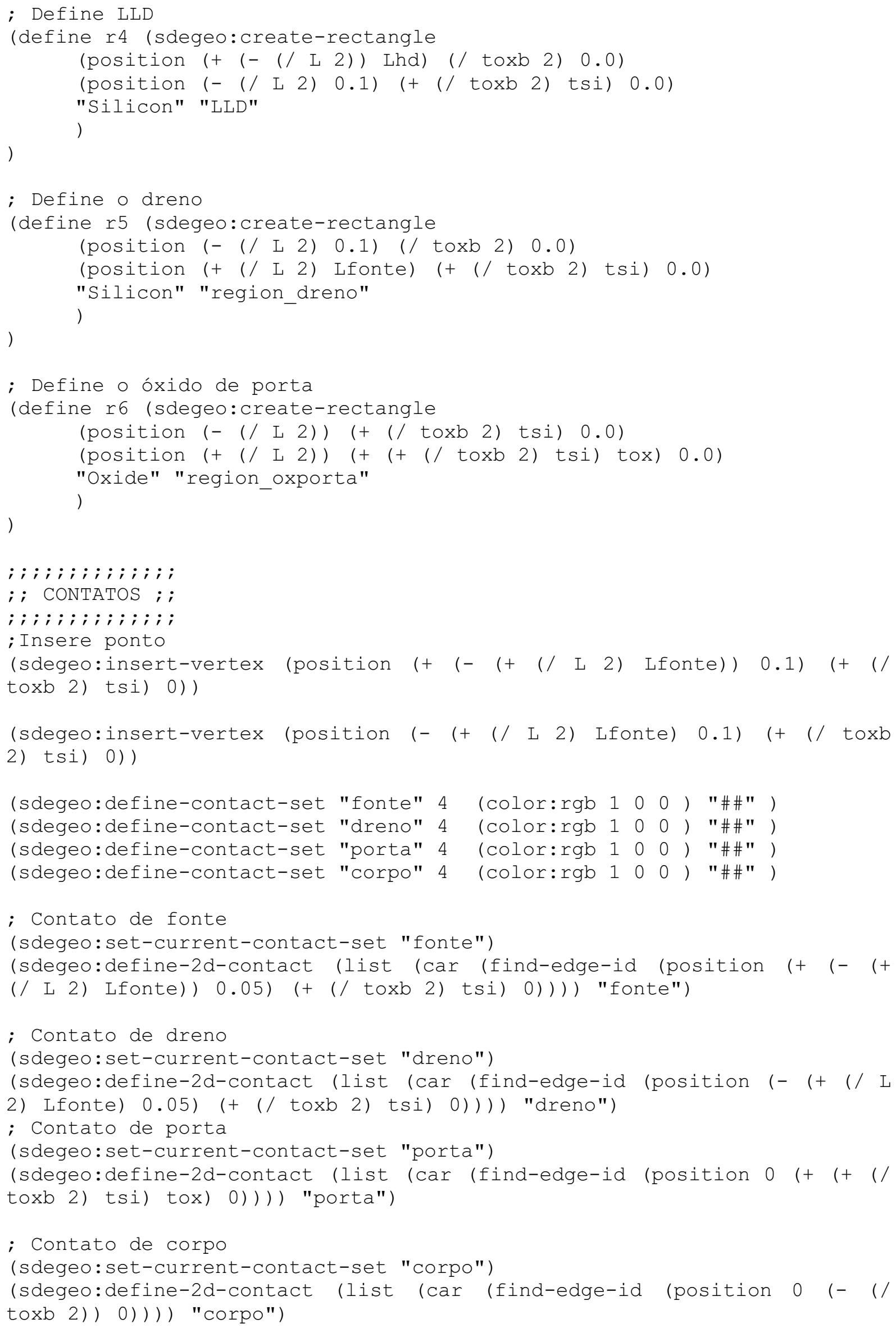




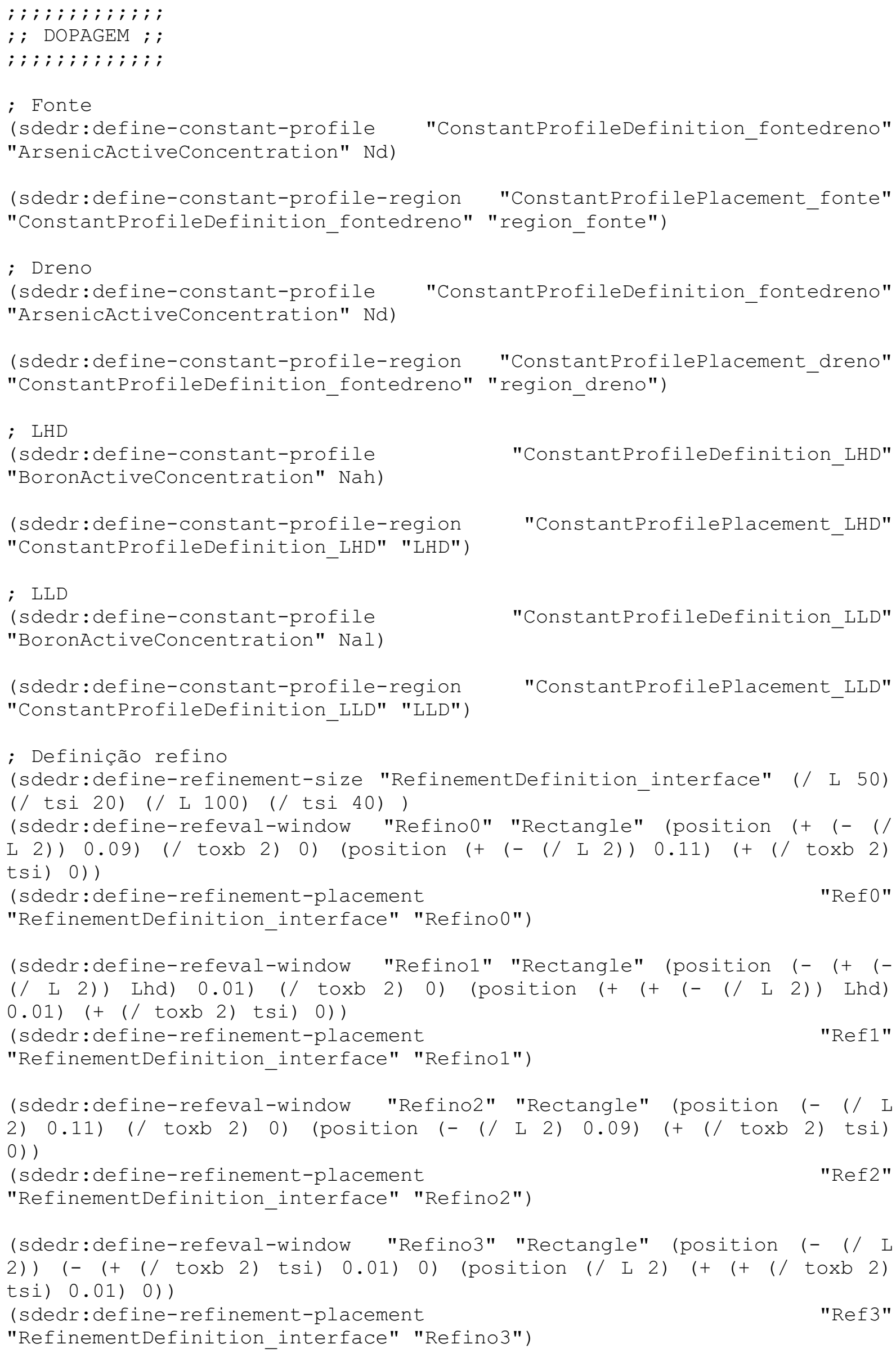


(sdedr:define-refeval-window "Refino4" "Rectangle" (position (- (/ L 2) ) (- (/ toxb 2) 0.01) 0) (position (/ L 2) (+ (/ toxb 2) 0.01$) 0)$ ) (sdedr: define-refinement-placement

"RefinementDefinition_interface" "Refino4")

"Ref 4 "

(sdedr:define-refinement-size "RefinementDefinition_fontedreno" (/ Lfonte 20) (/ tsi 10) (/ Lfonte 40) (/ tsi 20) )

(sdedr:define-refinement-region "RefinementPlacement_fonte" "RefinementDefinition_fontedreno" "region_fonte" )

(sdedr:define-refinement-size "RefinementDefinition_fontedreno" (/ Lfonte 20) (/ tsi 10) (/ Lfonte 40) (/ tsi 20) )

(sdedr:define-refinement-region "RefinementPlacement_dreno" "RefinementDefinition_fontedreno" "region_dreno" )

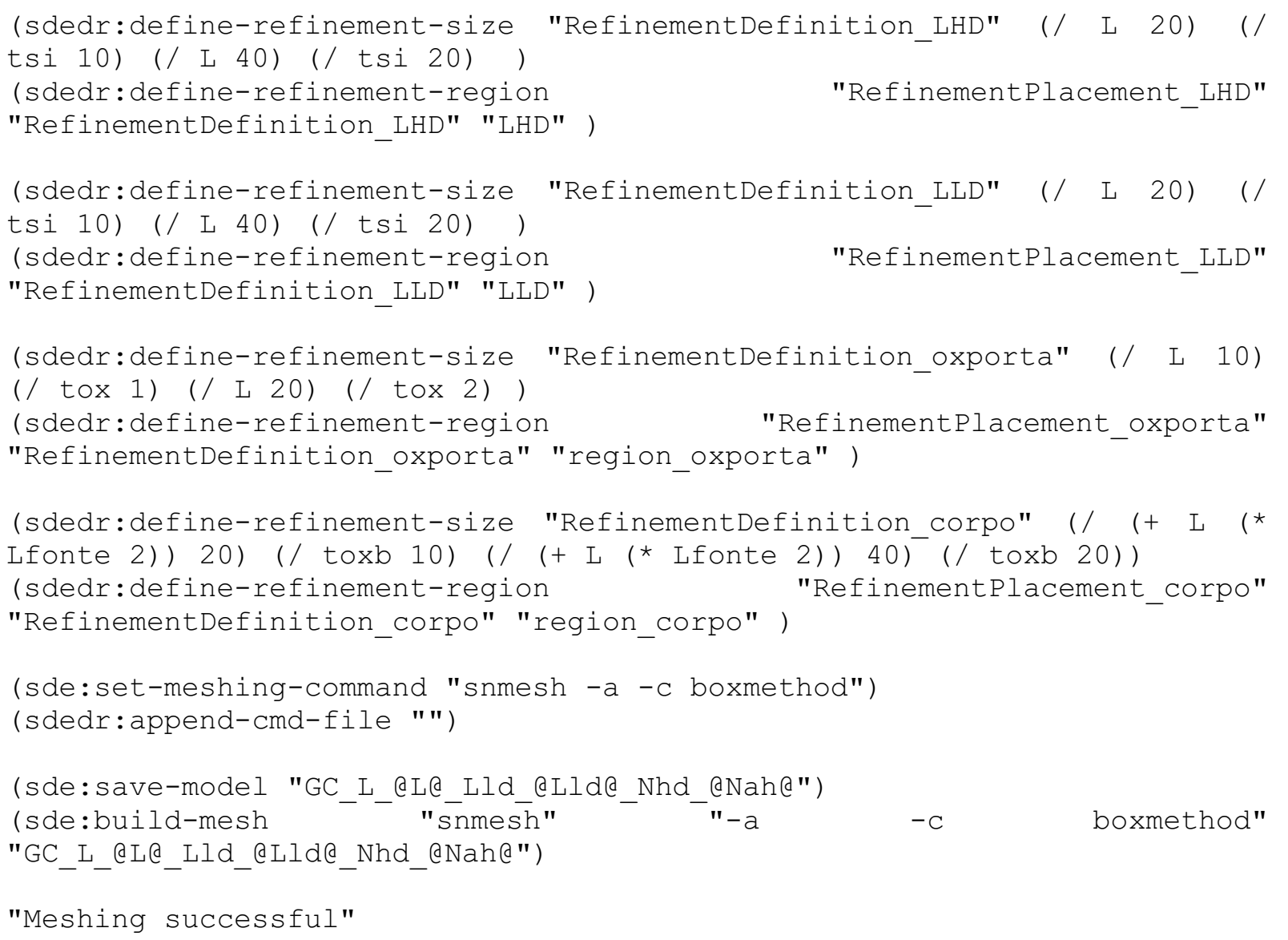

\section{Arquivo de simulação $\mathrm{I}_{\mathrm{DS}} \mathrm{x} \mathrm{V}_{\mathrm{GS}}$ de dispositivo no Sentaurus Device.}

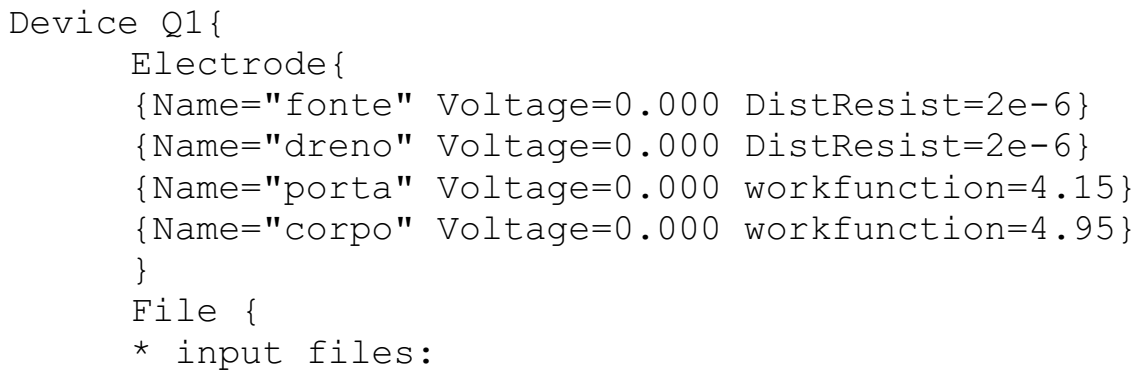




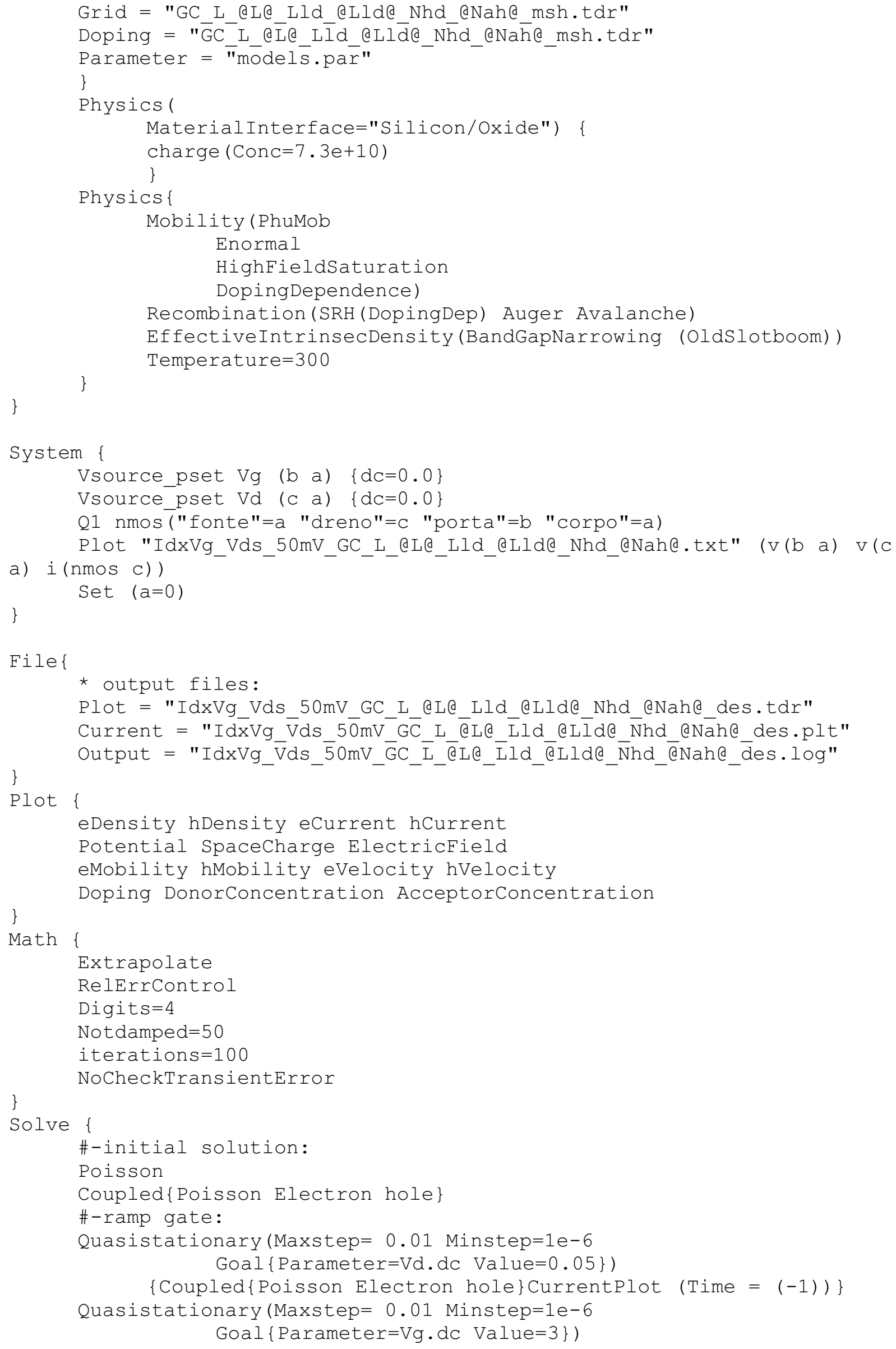


\{Coupled Poisson Electron hole\}CurrentPlot (Time $=($ range $=(0$ 1) intervals $=3000)$ ) \}

\} 


\section{Arquivo de simulação IDS X VDS de dispositivo no Sentaurus Device.}

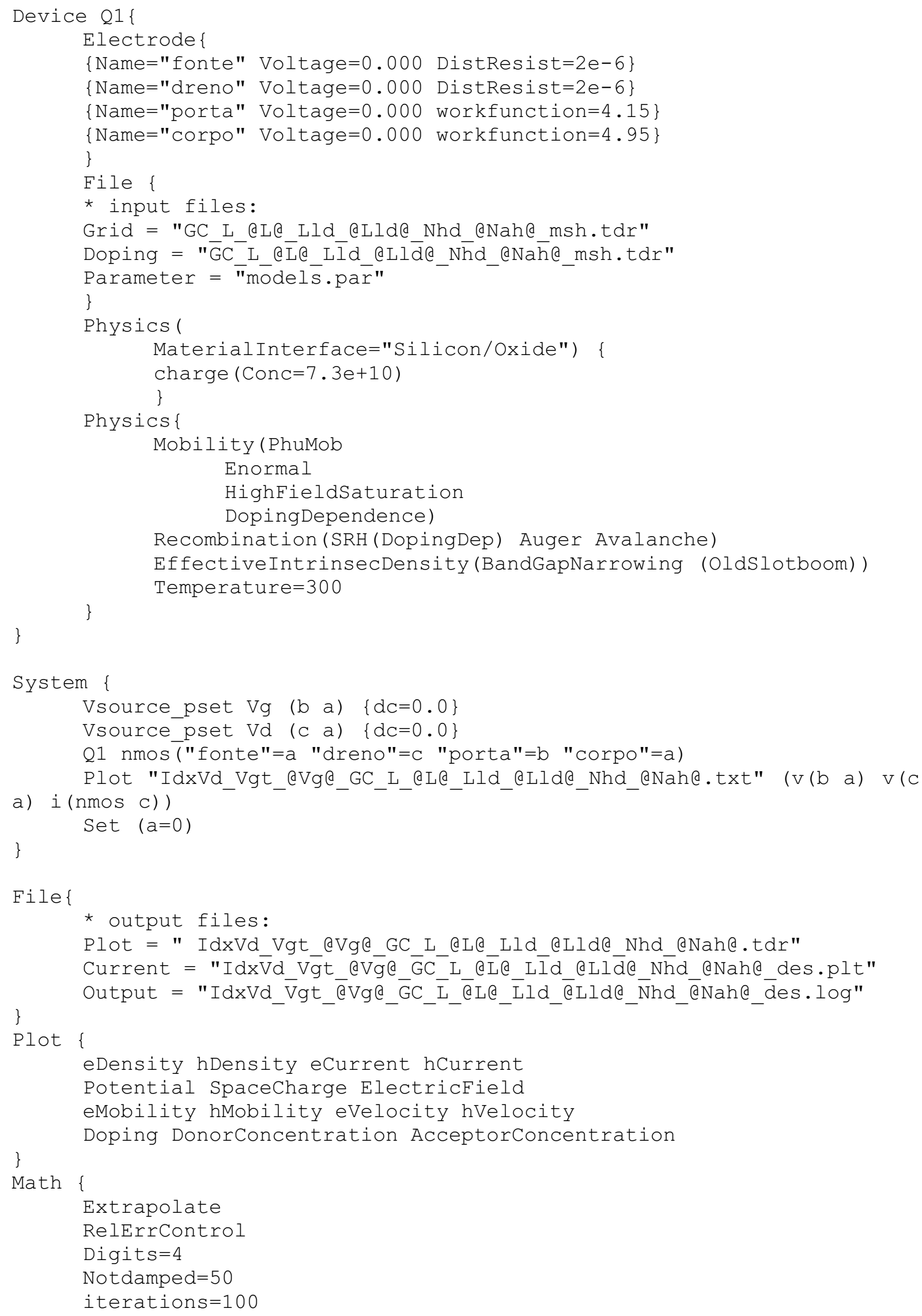




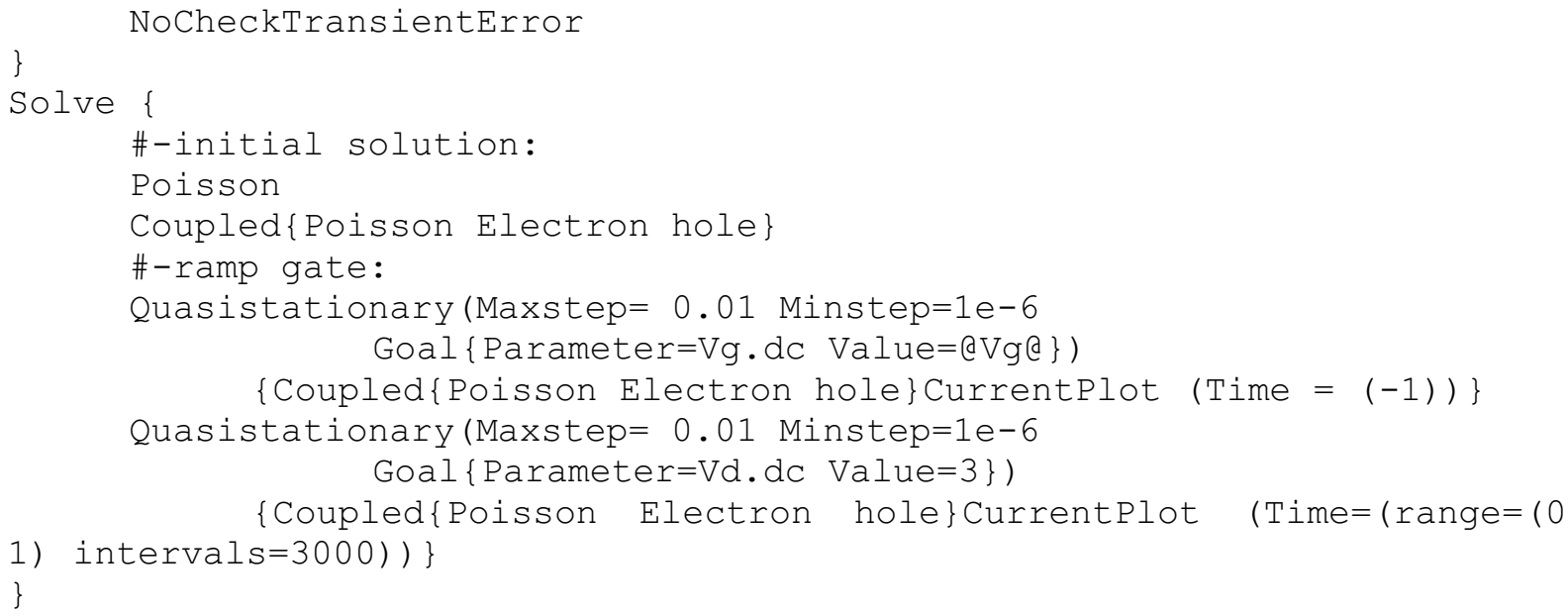




\section{Arquivo models.par para ajuste das simulações com os resultados experimentais:}

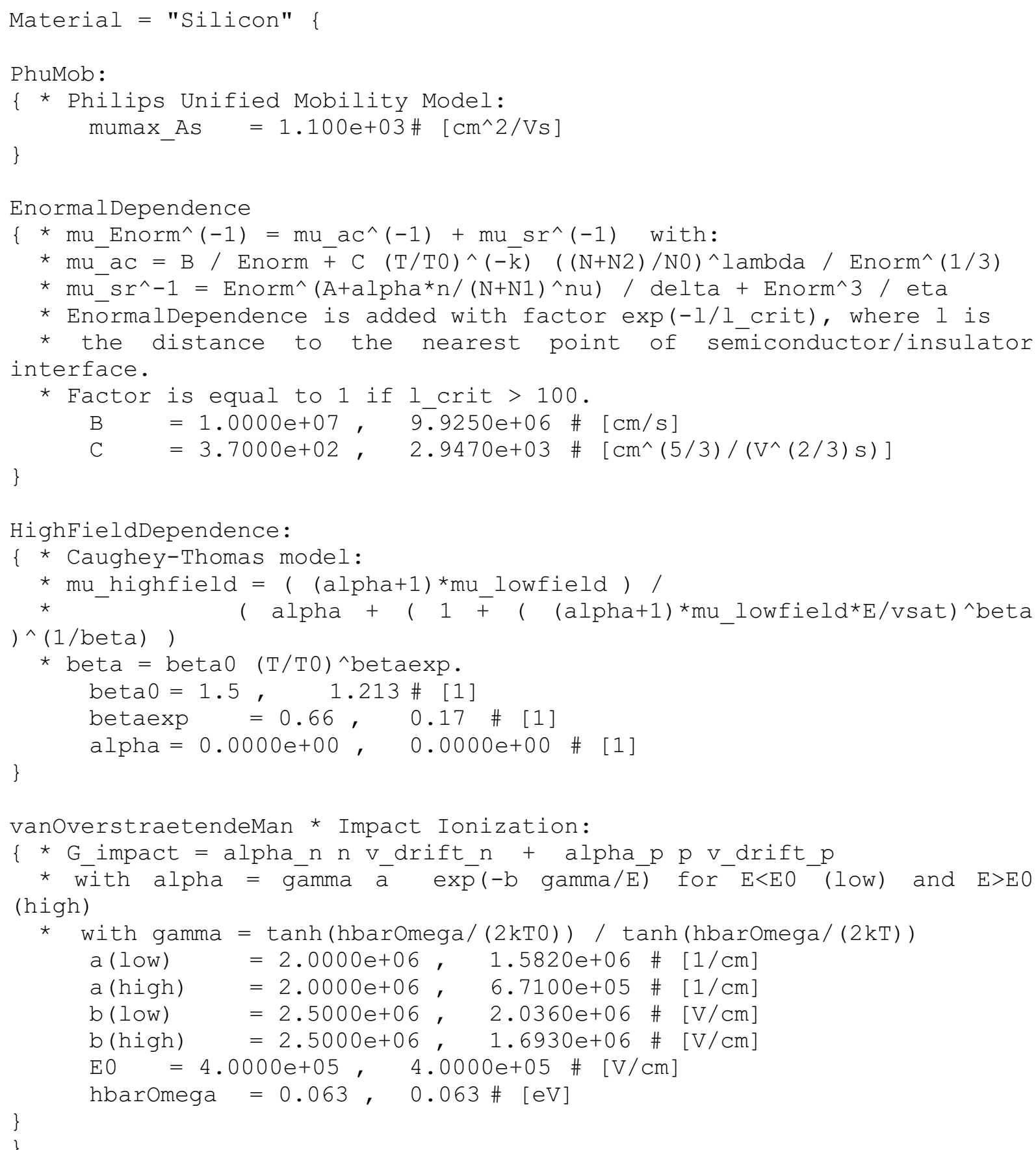


APÊNDICE C - TRABALHOS PUBLICADOS 
Os resultados obtidos neste trabalho permitiram a submissão e aceitação para apresentação oral do seguinte artigo:

"Experimental Evaluation of Mismatching on the Analog Characteristics of GC SOI MOSFETs"

Camila Restani Alves, Marcelo Antonio Pavanello, Denis Flandre e Michelly de Souza $32^{\text {nd }}$ Symposium on Microelectronics Technology and Devices. Fortaleza - Brasil 